\title{
PRECISION MØLLER POLARIMETRY AND APPLICATIONS AT JEFFERSON LABORATORY
}

\author{
A Dissertation \\ Submitted to
}

the Temple University Graduate Board

In Partial Fulfillment

of the Requirements for the Degree

DOCTOR OF PHILOSOPHY

\author{
by \\ William Henry \\ May 2019
}

Examining Committee Members:

Jim Napolitano, Department of Physics, Advisory chair

Andreas Metz, Department of Physics

Bernd Surrow, Department of Physics

Donald Jones, Department of Physics

Harsh Deep Chopra, College of Engineering 


\section{ABSTRACT}

Jefferson Lab's cutting-edge parity-violating electron scattering program has increasingly stringent requirements for systematic errors. Beam polarimetry is often one of the dominant systematic errors in these experiments. A new Møller Polarimeter in Hall A of Jefferson Lab (JLab) was installed in 2015 and has taken first measurements for a polarized scattering experiment. Upcoming parity violation experiments in Hall A include CREX, PREX-II, MOLLER and SOLID with the latter two requiring $<0.5 \%$ precision on beam polarization measurements, a precision which has not been achieved to date. The polarimeter measures the Møller scattering rates of the polarized electron beam incident upon an iron target placed in a saturating magnetic field. The spectrometer consists of four quadrupoles and one momentum selection dipole. The detector is designed to measure the scattered and knock out target electrons in coincidence. Beam polarization is extracted by constructing an asymmetry from the scattering rates when the incident electron spin is parallel and antiparallel to the target electron spin. The largest systematic errors associated with Møller polarimetry comes from the precision that the target polarization and the detector acceptance is known will be discussed. Other errors including the Levchuk effect, beam stability, and target heating will be addressed. 


\section{ACKNOWLEDGEMENTS}

It has been quite a journey since I started at Buck County Community College 20 years ago until now. The help, support, and motivation I have received from the countless people has been very appreciated and I wouldn't be here without them. Im grateful for the inspiration provided by my high school physics teacher, Mr. Finkbinder, along with my community college professor, Dr. Klein, they were the reason I chose to pursue physics. My family has always been supportive, whether it be my Mom's constant reminder on how important my education was or my father constantly taking me back to work at his iron shop so I could save up some money to take a few more classes. Equally supportive has been Raouf and Karen for providing me with a place to eat, work and live over the years. A special thanks is also due for my thesis advisor, Jim Napolitano. Jim has made my time at Temple rewarding, my work feel appreciated and made it easy to find the desire and will to continue on with my studies. He has always been available and eager to give advice, share his immense knowledge and provide guidance. I would also like to thank Don Jones for being a mentor and an integral part of nearly all the work I have done. I could have never finished without the countless hours he spent helping me in his office. The folks at JLab have also been tremendously helpful. Thank you Javier Gomez and Ethan Becker for giving me the opportunity to work with you in Hall A on the Møller polarimeter system. Thanks to Sasha Glamazdi and Roman Pomatsalyuk for passing on the knowledge that they have acquired over the years while taking Møller measurements. It was also a pleasure working with the entire polarimetry team that has formed since I have started. From Sanghwa Park and Eric King with the simulation effort to Dave Gaskell who was always willing to take time when I would stop by his office unannouced to answer my many questions. Thanks to Kent Paschke and Paul Souder for always asking the right questions, which forced me to work through some important issues that otherwise I may have overlooked. Additional thanks to James Wilhemi, Simona Malace, Jay Benish, Thia Keppel, The DVCS collaboration, Doug Higginbotham, my committee and everyone else that have contributed to my thesis. 


\section{CONTENTS}

$\begin{array}{lc}\text { ABSTRACT } & \text { i }\end{array}$

ACKNOWLEDGEMENTS ii

LIST OF FIGURES v v

LIST OF TABLES $\quad$ xvi

1 PHYSICS WITH GEV POLARIZED ELECTRONS 1

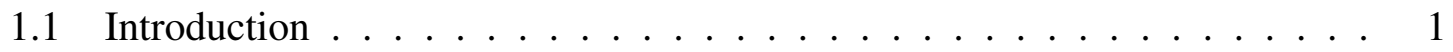

1.2 Nuclear Structure in Parity Conserving Reactions . . . . . . . . . . . 5

1.3 Parity Violation for Fundamental Interactions . . . . . . . . . . . 7

1.4 Parity Violation for Nucleon Structure $\ldots \ldots \ldots \ldots$

1.5 Upcoming experiments in Hall A at Jefferson Laboratory . . . . . . . . 10

2 PRECISION ELECTRON BEAM POLARIMETRY TECHNIQUES 14

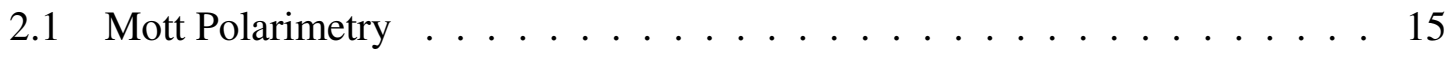

2.2 Compton Polarimetry . . . . . . . . . . . . . . . . . . . 18

2.3 Møller Polarimetry with Polarized Foil Target . . . . . . . . . . . . 22

2.3.1 Polarized Møller Scattering _. . . . . . . . . . . . . . . 23

2.3 .2 Key Elements . . . . . . . . . . . . . . . . . . . . 25

3 THE HALL A MØLLER POLARIMETER APPARATUS AND MODELING 32

3.1 Superconducting Split Coil Magnet . . . . . . . . . . . . . . 35

3.2 Target Motion System . . . . . . . . . . . . . . . . . . 38

3.3 Quadrupoles . . . . . . . . . . . . . . . . . . . 42

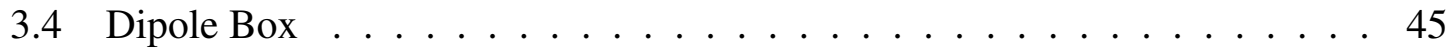

3.5 Detector Box $\ldots \ldots \ldots \ldots \ldots \ldots \ldots \ldots$ 
3.6 Geant3 Simulation . . . . . . . . . . . . . . . . . . . . . . . . . 49

3.6 .1 Physics Processes . . . . . . . . . . . . . . . . . . . . . . . 49

3.6 .2 Statistical Error . . . . . . . . . . . . . . . . . . . . 49

3.6.3 Data Comparison . . . . . . . . . . . . . . . . 50

3.6 .4 Geant4 Comparison . . . . . . . . . . . . . . . 52

4 UNCERTAINITIES IN MØLLER POLARIMETRY 58

4.1 Statistical Errors . . . . . . . . . . . . . . . . . . . . . . . . 59

4.2 Dead Time Corrections . . . . . . . . . . . . . . . 66

4.3 False Asymmetries . . . . . . . . . . . . . . 68

4.4 Target Polarization . . . . . . . . . . . . . . . . . 69

4.4.1 Compilation of World Data on Magnetization and g' . . . . . . 73

4.4.2 Target Angle Dependence . . . . . . . . . . . . . . 77

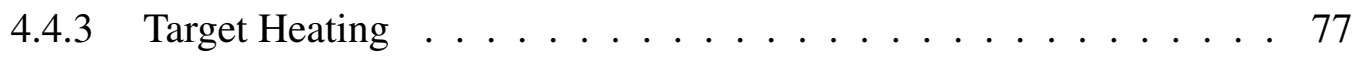

$4.4 .4 \quad$ XMCD analysis . . . . . . . . . . . . . . . . . . . 78

4.5 Analyzing Power $\ldots \ldots \ldots \ldots \ldots \ldots \ldots$

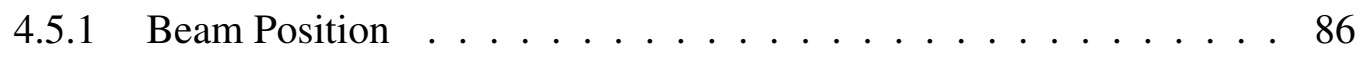

4.5 .2 Beam Energy . . . . . . . . . . . . . . . . 86

4.5 .3 Detector Geometry . . . . . . . . . . . . . . . . . . . 89

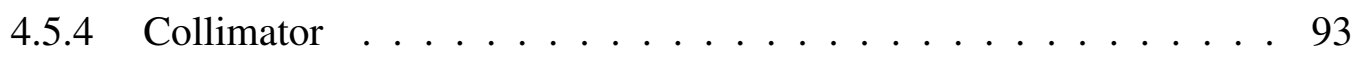

4.5.5 Multiple Scattering . . . . . . . . . . . . . . . . 993

4.5.6 Magnetic Fields and Alignment _. . . . . . . . . . . 93

4.6 Radiative Corrections . . . . . . . . . . . . . . . . . . . . . . 95

4.7 Levchuk Effect . . . . . . . . . . . . . . . . . . 95

5 POLARIZATION MEASUREMENTS AFTER THE UPGRADE 101

$5.1 \quad$ DVCS Results . . . . . . . . . . . . . . . . . . . 101

5.22019 Commisioning Run . . . . . . . . . . . . . . . 106

6 FUTURE PLANNED EXPERIMENTS IN HALL A 112

6.1 PREX-II and CREX . . . . . . . . . . . . . . . . . . . . . 112

6.1 .1 Operation at $02.2 \mathrm{GeV} \ldots \ldots \ldots \ldots . \ldots \ldots 112$

6.1 .2 Operation at $0.95 \mathrm{GeV} \ldots \ldots \ldots \ldots . \ldots \ldots$

6.2 Polarimetry for MOLLER and SoLID . . . . . . . . . . . 123

$\begin{array}{ll}\text { BIBLIOGRAPHY } & 127\end{array}$ 


\section{LIST OF FIGURES}

1.1 Kinematic variables in a transfer polarization experiment when the recoil polarization is measured(left) and when the target is polarized(Right) [1] .

1.2 (Left) Demonstration of Rosenbluth separation at $Q^{2}$ values of 2.5 (open triangle), 5.0 (circles), 7.0 (filled triangles) $\mathrm{GeV}^{2}$ [2] (Right) Comparison of the ratio of $G_{E p} / G_{M p}$ by using Rosenbluth separation and polarization transfer data.[1] . . . . . . . . . . . . . . . . . . . . 6

1.3 Measured asymmetry in E122. Each run was 1 to 3 hours and the solid line is the expected result $[3] \ldots \ldots \ldots \ldots$

1.4 Upcoming experiments in Hall A (CREX, PREX-II, MOLLER, SoLID) will be pushing the frontier by measuring extremely small asymmetries with remarkable precision (Figure courtesy of Kent Paschke) . . . . . . . . 11

2.1 Sherman Functions for Gold nucleus. These function show the asymmetry in a Coulomb field as a function of scattering angle when assuming a point like nucleus and no screening. $[4] \ldots \ldots \ldots$

2.2 Foil - thickness extrapolation for 2.75, 5 and $8.2 \mathrm{MeV}$. Lines symbolize calculation and dots the measurement. [5] . . . . . . . . . . . . . 17 
2.3 MeV Mott Polarimeter at Jefferson Lab. Electron is incident from right, scatters off target foil, and is detected after passing through the aluminum windows . . . . . . . . . . . . . . . . . . . 17

2.4 Compton Scattering [6] . . . . . . . . . . . . . . . . 18

2.5 Compton Scattering Cross Section (left) and longitudinal analyzing power (right) assuming a $532 \mathrm{~nm}$ laser colliding with an electron beam at $1 \mathrm{GeV}$, $11 \mathrm{GeV}$ and $27 \mathrm{GeV} .[6]$. . . . . . . . . . . . . . . . . . 20

2.6 Compton Polarimeter in Hall A [7] . . . . . . . . . . . . . . . . . . 20

2.7 (Left)Compton photon energy spectrum (Right)Asymmetry vs photon energy spectrum. Both plots show data fit to Monte Carlo simualtion [8] . . . 21

2.8 Integrating mode asymmetry for different circular polarization laser states and with the laser cavity unlocked (for background subtraction)Here $M^{+(-)}$ is the measured integrated signal plus background for positive (negative) helicity electrons. [8] . . . . . . . . . . . . . . . 22

2.9 a) Scheme of "classic" technique of ferromagnetic foil polarization in the foil plane. b) Scheme of "brute force" technique of ferromagnetic foil polarization in out-of-the-foil plane.[9] . . . . . . . . . . . . . . . . . 23

2.10 The Møller scattering angle in degrees of the lab frame vs the CM frame . . 26

2.11 The Møller polarimeter in Hall C(top). Collimation region (bottom) . . . . 27

2.12 Result of the Hall A electron beam polarization measurements with Møller and Mott polarimeter for experiment E-93-027. This was the first reported results for the Møller polarimeter in Hall A. The Møller polarimeter initially reported the uncertainty (statistical and systematic) in a polarization measurement at $\leq 3 \%[10] \ldots \ldots$. . . . . . . . . . . . 29 
2.13 Polarization results before and after the upgrade. Measurements taken in Hall A of Jefferson Lab for The DVCS experiment . . . . . . . . . 30

3.1 Hall A Møller Polarimeter at Jefferson Laboratory as modeled in Geant3. To give the reader an idea of the aspect ratio and scale, the quadrupoles have a circular cross-section with an outer diameter of $10 \mathrm{~cm}$ and the entire setup from target to detector is about $7 \mathrm{~m}$ long . . . . . . . . . . . . 33

3.2 (Left) The magnetic field strength inside the bore of the split coil magnet(right). To determine the magnetic axis of the magnet the transverse minimum or maximum at different $\mathrm{z}$ locations were determined from magnetic field mapping measurements. . . . . . . . . . . . . 36

3.3 Measured data and fit of holding field magnet. Once the fit was determined the radial component could be computed. The red line shows the radial component at $1.27 \mathrm{~cm}$ off from the z-axis. The target foils are located at $\mathrm{z}=0 \mathrm{~cm} \ldots \ldots \ldots \ldots \ldots \ldots$

3.4 By including offsets and rotations to each of the coils, the magnet mapping data could be explained. The figure on the left had one coil shifted $0.4 \mathrm{~mm}$ and rotated $0.25^{\circ}$ and the figure on the right has both coils shifted $0.67 \mathrm{~mm}$ and rotated $0.23^{\circ}$. Ideal coils that were perfectly aligned would have a line at $x=0$ and $y=0$. The discontinuities are at the points where the saddle point changes from negative to positive curvature and have essentially 0 slope everywhere so there is no sensitivity to find a precise location in $\mathrm{x}$ and $\mathrm{y} . \quad$. 37

3.5 Coincidence and single electrons on detector at $0.95 \mathrm{GeV} \ldots \ldots . \ldots 38$

3.6 Temple University Møller Target Motion System in the design phase (2016). Engineered and drawn by James Wilhemi $\ldots \ldots$. . . . . . . . . . 40

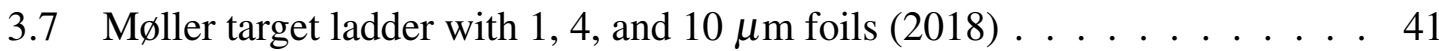


3.8 New Møller target system installed on the superconducting split coil mag$\operatorname{net}(2017) \ldots \ldots \ldots \ldots \ldots \ldots$

3.9 Main components of target motion system which allow rotation and translation of the target foils. Two feed-thru's are required since the target is under vacuum and the motors are located outside vacuum. An additional rotational encoder was added and not shown here. . . . . . . . . . . . . . . 41

3.10 The magnet field of an ideal quadrupole and force it exerts on a moving charged particle[11] . . . . . . . . . . . . . . . 42

3.11 Suggested quadrapole settings as a function of electron beam energy ht $t \mathrm{ps}$ : //hallaweb.jlab.org/equipment/moller/docs/upgrade_ $11 \mathrm{gev} . \mathrm{pdf} \ldots \ldots \ldots \ldots \ldots$

3.12 Results when using the solution in Figure 3.11 to determine quadrupole settings at various nominal beam energy settings . . . . . . . . . . . . . . . 44

3.13 Determination of the effective length used in the simulation from mapping measurements [12] . . . . . . . . . . . . . . . 45

3.14 Dipole entrance and collimators . . . . . . . . . . . . . 46

3.15 Lead shielding at dipole exit . . . . . . . . . . . . . . . 47

3.16 Detector Box in Geant3 . . . . . . . . . . . . . . . . . . . . . 48

3.17 Geant3 Møller weighted generator for $65^{\circ} \leq \theta_{C M} \leq 115^{\circ}$. Electron pairs that were accepted by the detector are in the filled area labeled coincidence. $\quad 50$

3.18 Coincidence pairs detected and $A_{z z}$ from simulation for $100 \mathrm{k}$ generated events. Each run of the simulation was done with a different random seed in order to approximate the statistical error. . . . . . . . . . . . . . 51 
3.19 2015 optics scan at $2.056 \mathrm{GeV}$ and Geant3 comparison (Q3 is turned off). Note that the single point in the Q1 scan at $0.6 \mathrm{kG}$ is suspect. . . . . . . . 52

3.20 Geant3(bottom)/Geant4(top) Comparison. Tracking the electrons through the quadrupole region of the beam pipe. Color scale is $\theta_{C M}$ for RHS electrons 54

3.21 GEANT3 vs GEANT4 ray tracing comparison at split coil target magnet exit. 55

3.22 Geant3(bottom)/Geant4(top) ray tracing comparison. (Top)Magneta dashed lines indicate locations of virtual planes. (Bottom) Difference in position at planes between two simulations . . . . . . . . . . . . 56

3.23 Geant3(bottom)/Geant4(top) Comparison. (Top) $A_{z z}$ at beam energy of 2.2 GeV. (Bottom) Difference in number of electrons in each $A_{z z}$ bin. . . . . . . 57

4.1 Asymmetry measurement for run 16159. Each entry corresponds to one quartet or two $N_{+}$and two $N_{-}$cycles . . . . . . . . . . . . . 61

4.2 Output of analysis script for run $16159 \ldots \ldots$. . . . . . . . . 62

4.3 Time required to reach level of relative uncertainty versus coincidence rate. 63

4.4 Relative statistical uncertainty versus length of run for various coincidence rates. . . . . . . . . . . . . . . . . . . . 64

4.5 Coincidence rates for various runs in 2016 during DVCS . . . . . . . . 65

4.6 Data from dedicated LED pulser runs to determine dead time correction . . 67

4.7 Magnetization of Fe from [13] using force method . . . . . . . . . . 70

4.8 Iron magnetizations from Graham. Colored bars represent the values used

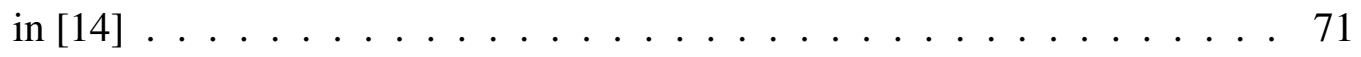


4.9 High field dependence of magnetization. The $\mathrm{x}$-axis is the internal field which is related to the applied field according to Equation 4.29 [15] . . . . 72

4.10 Work data on Fe magnetization $[16] \ldots \ldots$. . . . . . . . . 74

4.11 Fit of world data for $\mathrm{Fe}[16] \ldots \ldots \ldots 75$

4.12 Fit of world data for $\mathrm{Ni}[16] \ldots \ldots \ldots$. . . . . . . . . . . 75

4.13 Fit of world data for $g$ of Fe $[16] \ldots \ldots \ldots \ldots$

4.14 Fit of world data for $g^{\prime}$ of $\mathrm{Fe}[16] \ldots \ldots \ldots \ldots$

4.15 Recommended vales for Fe Target Polarization values for Møller Polarime-

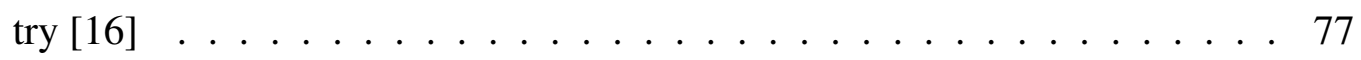

4.16 Relative foil saturation vs applied field for various angle relative to the applied field. . . . . . . . . . . . . . 78

4.17 CFD calculation of target heating by Silviu Covrig. Assumes $2 \mu \mathrm{A}$ beam, $90 \mu \mathrm{m}$ spot size, on a $10 \mu \mathrm{m}$ Fe foil with a 10 inch diameter which results in a $35 \mathrm{~K}$ increase in temperature. . . . . . . . . . . . . . . . . 79

4.18 Correction to apply to magnetization as a function of beam current . . . . 79

4.19 Analysis of XAS(left) and XMCD(right) spectrum using the sum rules . . . 81

4.20 Left: Integration(blue curve) without shift Right:Integration with spectrum shifted vertically ..................... 82

$4.21 \frac{m_{o}}{m_{s}}$ dependence on the location of $\mathrm{p}$ and $\mathrm{q} \ldots \ldots . \ldots 3$

4.22 Data fit to Voigt functions . . . . . . . . . . . . . 84 
4.23 Various oxide contributions to iron foil spectrum $\ldots \ldots \ldots \ldots$. . . 84

4.24 The magnetization and orbital to spin magnetic moment ratio from Mössbaurer spectroscopy and a magnetic compton profile.Cite The purple lines show our values calculated from the sum rules at .5T . . . . . . . . . 85

4.25 Acceptance and analyzing power for $E_{B E A M}=2.22 \mathrm{GeV}$. The color scale on the left represents the number of electrons in each bin with blue being the fewest and the redder regions being the most. $\ldots \ldots \ldots 86$

$4.26 \mathrm{x}$ beam position dependence on $\left\langle A_{z z}\right\rangle$ and rates. The width of the green box represents the uncertainty of the $\mathrm{x}$-axis and the height the corresponding uncertainty of $\left\langle A_{z z}\right\rangle \ldots \ldots \ldots \ldots \ldots \ldots \ldots \ldots \ldots$

$4.27 \mathrm{y}$ beam position dependence on $\left\langle A_{z z}\right\rangle$ and rates. The width of the green box represents the uncertainty of the $\mathrm{x}$-axis and the height the corresponding uncertainty of $\left\langle A_{z z}\right\rangle \ldots \ldots \ldots \ldots \ldots \ldots \ldots \ldots$

4.28 Beam energy/momentum dependence on $\left\langle A_{z z}\right\rangle$ and rates. The width of the green box represents the uncertainty of the $\mathrm{x}$-axis and the height the corresponding uncertainty of $\left\langle A_{z z}\right\rangle \ldots \ldots \ldots \ldots \ldots$

4.29 (Left) Coincidence electron locations on the calorimeter blocks(grey) color coded by energy $\left(\theta_{C M}\right)$. The blue electrons are the highest energy while the red are the lowest. The red dashed line is the outline of the opening in the lead shielding that surrounds the detector.(Right) Side view of the calorimeter, entrance opening, and lead shielding box. The position of the detector and entrance were separately shifted in y (up and down in figure) and $x$ (in and out of page) and the change in the analyzing power and rates were found. . . . . . . . . . . . . . . . . . 89

$4.30 \mathrm{x}$ detector position dependence on $\left\langle A_{z z}\right\rangle$ and rates. . . . . . . . . . 90 
4.31 y detector position dependence on $\left\langle A_{z z}\right\rangle$ and rates. The width of the green box represents the uncertainty of the x-axis and the height the correspond-

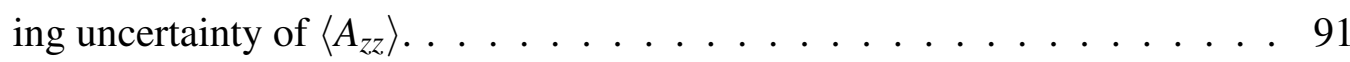

$4.32 \times$ detector entrance position dependence on $\left\langle A_{z z}\right\rangle$ and rates. The width of the green box represents the uncertainty of the $\mathrm{x}$-axis and the height the corresponding uncertainty of $\left\langle A_{z z}\right\rangle \ldots \ldots \ldots \ldots$. . . . . . 91

4.33 y detector entrance position dependence on $\left\langle A_{z z}\right\rangle$ and rates. The width of the green box represents the uncertainty of the $\mathrm{x}$-axis and the height the corresponding uncertainty of $\left\langle A_{z z}\right\rangle \ldots \ldots \ldots \ldots$. . . . . . 92

4.34 Collimator opening dependence on $\left\langle A_{z z}\right\rangle$ and rates. The width of the green box represents the uncertainty of the x-axis and the height the corresponding uncertainty of $\left\langle A_{z z}\right\rangle \ldots \ldots \ldots \ldots \ldots \ldots \ldots \ldots$

4.35 The effects of multiple scattering for different foil thickness on $\left\langle A_{z z}\right\rangle$ and rates. . . . . . . . . . . . . . . . . . . . 994

4.36 Key elements in Geant3 simulation of Møller Polarimeter . . . . . . . . 95

4.37 $\theta_{C M}=90^{\circ}$ Møller scattered electron trajectories when scattered from different atomic shells in the Hall A polarimeter. The inner shells tend to smear the angular distribution resulting in an increase of the effective tar-

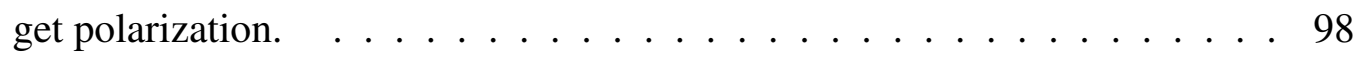

4.38 The Levchuk correction as a function of the magnetic field of Q2. This particular tune is for a $2.1 \mathrm{GeV}$ beam. The structure of the correction at the lower fields can provide a check for our understanding of the effect. . . . 100

5.1 Beam polarization measurements before and after the upgrade of the Hall

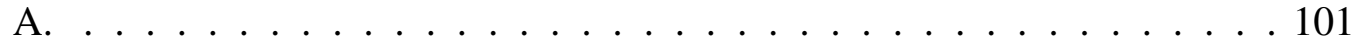


5.2 The Møller polarimetry systematic errors during E12-06-114 which used the tilted and brute-force target. . . . . . . . . . . . . . . . 102

5.3 Measured longitudinal polarization delivered to Hall A as a function of Wein angle set at source . . . . . . . . . . . . . . . . 103

5.4 Measured polarization as a function of the target holding field. Ideally the target foil would be at exactly $90^{\circ}$ and the foil would reach maximum polarization at 2.2T . . . . . . . . . . . . . . . . . . . . 104

5.5 Quad scans performed in 2016. The red triangles have been corrected for the Levchuk effect. . . . . . . . . . . . . . . . . . . . . . . . 105

5.6 Polarization measurements during 2019 commissioning. The statistically lower polarization measured with the $1 \mu \mathrm{m}$ foil could be a result of wrinkles or deformations in the foil. . . . . . . . . . . . . . . . 107

5.7 (Top)Current normalized scalars averaged every 4 seconds for both helicity cycles. (Bottom) Asymmetry averaged every 4 seconds $(30 \mathrm{~Hz}$ helicity flip

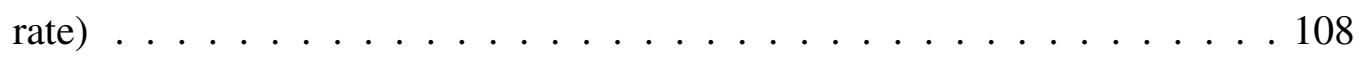

5.8 Polarization measurements while changing the target foil angle . . . . . 109

5.9 2019 Dipole and Q1 Scan at 2.139 GeV. Q3 is off. Plots courtesy of Eric King. ........................... 110

5.10 2019 Q2 and Q4 Scan at 2.139 GeV. Q3 is off. Plots courtesy of Eric King. 111

6.1 Coincidence pairs plotted as Q4 field vs Q2 field for various values of Q1. The redder regions indicate higher rates and possible solutions for a beam energy of $2.2 \mathrm{GeV} \ldots \ldots \ldots$. . . . . . . . . . . . . . . . . . . . . . . . . . . 
6.2 Q2 Quad scan at 2.2 GeV. This is a one quadrupole solution with the other three quadrupoles turned off. Coincidence (Top), Analyzing Power(Middle), Levchuk Correction(Bottom) . . . . . . . . . . . . . . . . . . . 114

6.3 Three quadrupole $2.139 \mathrm{GeV}$ solution. This solution also can be used for $2.2 \mathrm{GeV}$ (CREX). Q1=2.5 kG, Q2=0.5 kG, Q3=0 kG and Q4=1.0kG . . 115

6.4 Effect of detuning optics components on analyzing power and coincidence rates at $2.2 \mathrm{GeV}$ (CREX). The $\pm 0.5 \%$ and $\pm 0.1 \%$ error bars on right are

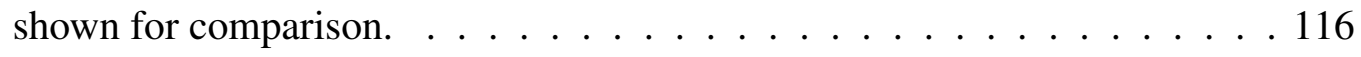

6.5 Coincidence electrons on detector plane for CREX and PREX spectrometer

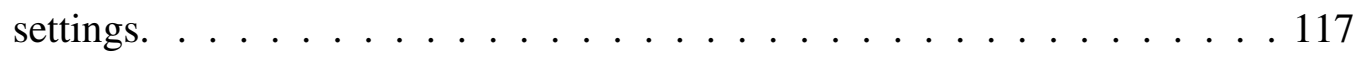

6.6 Phase Space Scan of Q1, Q2, and Q4 at $0.95 \mathrm{GeV} \ldots \ldots \ldots \ldots \ldots$

6.7 Phase Space Scan of Q1, Q2, and Q4 at $0.95 \mathrm{GeV} \ldots \ldots \ldots \ldots \ldots$

6.8 Higher resolution phase space scan of Q1, Q2, and Q4 at $0.95 \mathrm{GeV} \ldots \ldots 120$

6.9 Effect of detuning optics components on analyzing power and coincidence rates at $0.95 \mathrm{GeV}$ (PREX). The $\pm 0.5 \%$ and $\pm 0.1 \%$ error bars on right are shown for comparison. . . . . . . . . . . . . . . . . 121

6.10 Effect of detuning optics components on analyzing power and coincidence rates at $0.95 \mathrm{GeV}$. The $\pm 0.5 \%$ and $\pm 0.1 \%$ error bars on right are shown for comparison. This plot is the same as Figure 6.9 except the $\mathrm{x}$-axis is in percent versus Gauss . . . . . . . . . . . . . . . . 122 
6.11 The detector acceptance (in maroon) for MOLLER, CREX, and PREX beam energies. The color scale represents the z-location of the electron that died furthest upstream. For a beam energy of $11 \mathrm{GeV}$ (MOLLER) the acceptance is defined entirely on the dipole face. This can be seen by the green surrounding the maroon. At CREX and PREX energies the acceptance is also defined inside the dipole box (yellow/orange) and on the detector shielding box (red) . . . . . . . . . . . . . . . 125

6.12 Electron pairs generated in $\theta_{C M}$ from $100^{\circ}$ to $115^{\circ}$ and the complimentary electron from $65^{\circ}$ to $80^{\circ}$. The high energy electrons define the coincidence acceptance at the dipole face. . . . . . . . . . . . . . 126 


\section{LIST OF TABLES}

1.1 Example of experiments relying on polarimetry $\ldots \ldots \ldots \ldots$

2.1 Møller Polarimeters at various laboratories and their associated errors . . . 31

4.1 Error budget from MIE proposal to DOE for MOLLER experiment [17] . . 59

4.2 Foil polarization from $[14] \ldots \ldots \ldots$

4.3 Summary of results with referenced values coming from [18] $\ldots \ldots$. . 83

4.4 Error Budget Table for Møller Polarimetry at $2.2 \mathrm{GeV} \ldots \ldots \ldots$. . . . . . 99 


\section{CHAPTER 1}

\section{PHYSICS WITH GEV POLARIZED ELECTRONS}

\subsection{Introduction}

Humans have always striven to understand the composition of matter. Early observations were based off what we "saw", by making visual observations using light. However visible light or photons has limitations on what it can reveal because it behaves like a wave which can only resolve objects larger than its wavelength $(\sim 0.5-1.0 \mu \mathrm{m})$. De Broglie taught us that all particles have a wavelength, $\lambda$, which is inversely proportional to its momentum, $p$, according to the relation $\lambda=\frac{h}{p}$, where $h$ is Planck's constant. This allowed scientists to use other particles to probe matter at much smaller scales and began a push to develop higher energy accelerators so that physicists could study matter at shorter and shorter distances. An ideal particle to use like a precision microscope were electrons because they are pointlike, can be accelerated to high momentum (small wavelengths), and the physics processes they undergo are well understood by the laws of physics. Scattering experiments using electrons have been an important tool over the last 100 years by shedding new light on how we understand the fundamental building blocks of matter.

In order to appreciate the importance of particle physics and scattering experiments as a probe of matter at small scales, a brief review of the history is fruitful. In 1911, Ernest Rutherford explained a famous scattering experiment in which alpha particles were scattered off of a thin gold target. It was expected that the alpha particles would scatter at very small angles from the gold target which was believed to be comprised of a spatially uniform spread out positive "pudding". However, it was observed that most of the alpha 
particles passed through the foil with very little deflection and even more puzzling that a small fraction of them bounced back at very large angles. Rutherford concluded that matter was mostly empty space, with most of the mass of an atom located at a very dense center and that these centers were spaced very far apart relative to their size. From this experiment the famous Rutherford cross section was derived.

In 1915, Niels Bohr elaborated on this model of the atom as being a dense positive center surrounded by negative charge by quantizing the allowed energy levels of the electrons that circled the nucleus. He named the nucleus of the lightest atom, Hydrogen, the proton. His model was extremely successful at explaining the Rydberg formula which described the observed spectral lines of atomic hydrogen. Shortly thereafter, Wolfgang Pauli, Werner Heisenberg, and Erwin Schrödinger further improved the model upon the birth of quantum mechanics. The elementary structure of the atom was completed in 1932 when Chadwick discovered the neutron. From this point on, the atom, in its simplest form, is composed of positively charged protons and neutral neutrons at a dense center surrounded by a much larger cloud of negatively charged electrons.

The first modern particle accelerators were built in the 1950's like the Bevatron at Berkley, the Stanford Linear Accelerator (SLAC), and the Cosmotron at Brookhaven among others. As the energies of these accelerators exceeded $1 \mathrm{GeV}$ we were able to probe the nucleus of the atom with electrons that had wavelengths $<1 \mathrm{fm}$. We soon learned that the protons and neutrons (collectively known as nucleons) in the nucleus were made up of quarks. At these higher energies we were able to "see" the gluons and quark sea that make up the nucleons as well. GeV electrons also led to discoveries in deep inelastic scattering where the struck nucleon no longer stayed intact and then a whole new zoo of particles were discovered.

Over the last 40 years the demand for experiments using polarized electrons at $\mathrm{GeV}$ scales to study hadronic structure has been increasing. Experiments were proposed to study the elastic from factors of the nucleon and later the contributions that sea quarks make to them. Other experiments were focused on understanding the spin structure of the nucleon and how the quarks, gluons and their associated angular momentum add up to the spin $1 / 2$ particles that are the proton and neutron. There is also a whole class of Parity-Violating electron Scattering (PVeS) that aim to understand the electroweak force by measuring weak neutral form factors, neutron distributions in heavy nuclei, and search for contributions beyond the Standard Model of electroweak interactions. A common feature of all these 
experiments is a polarized beam in which the helicity is flipped many times per second allowing an asymmetry to be measured between the two different helicity states.

An experimental measured asymmetry is proportional to the product of the beam polarization and the "physics" asymmetry. Therefore, although statistical and systematic uncertainties inherent in the asymmetry measurement are always important, in the end, you don't want to be limited by the precision with which you know the polarization of the incident electron beam. High precision polarimetry is essential when measuring asymmetries and the motivation for this thesis.

Polarized electron scattering experiments can be broadly divided into two categories, parity conserving experiments and parity violating experiments. An important distinction between the two is the size of the measured asymmetry. Parity violation requires an interaction with a pseudo scalar term, for example the inner product between a spin and a momentum. In electron scattering, this means an interference between the weak neutral current and the electromagnetic current, which can be shown diagrammatically is proportional to $Q^{2} / M_{Z}^{2} \approx 120 \mathrm{ppm}$ at $Q^{2} \approx 1 \mathrm{GeV}$, where $Q$ is the momentum transfer and $M_{Z}$ is the mass of the Z-boson. For parity conserving processes, all amplitudes are of the same order, so the asymmetry is much larger, e.g. for Møller scattering it is almost unity (7/9).

A parity conserving experiment with a polarized beam almost always requires a second polarization measurement to be made in addition to measuring the beam polarization in order to be able to construct an asymmetry. Often the target is polarized and the measured asymmetry is

$$
A=\frac{N_{L L}-N_{R L}}{N_{L L}+N_{R L}}=P_{B} P_{t} \frac{\sigma_{L L}-\sigma_{R L}}{\sigma_{L L}+\sigma_{R L}}
$$

where $P_{B}$ is the beam polarization, $P_{t}$ is the target polarization, $N$ is the counting rates and $\sigma$ is the cross section. The subscripts $L$ and $R$ refer to the polarization or helicity with the first subscript referring to the beam and the second to the target. Another method of a parity conserving experiment exploits the polarization transfer from the incident electron to the unpolarized target and then the recoil particle polarization is measured. Both scenarios are expressed in Figure 1.1 In either case the precision which you know the recoil or target polarization is generally greater than the precision in which you know the electron beams polarization. Therefore in parity conserving experiments the precision of which you must know the beam polarization is usually determined by the precision of the target or recoil polarization measurement. In PVeS experiments the target is not polarized and the 

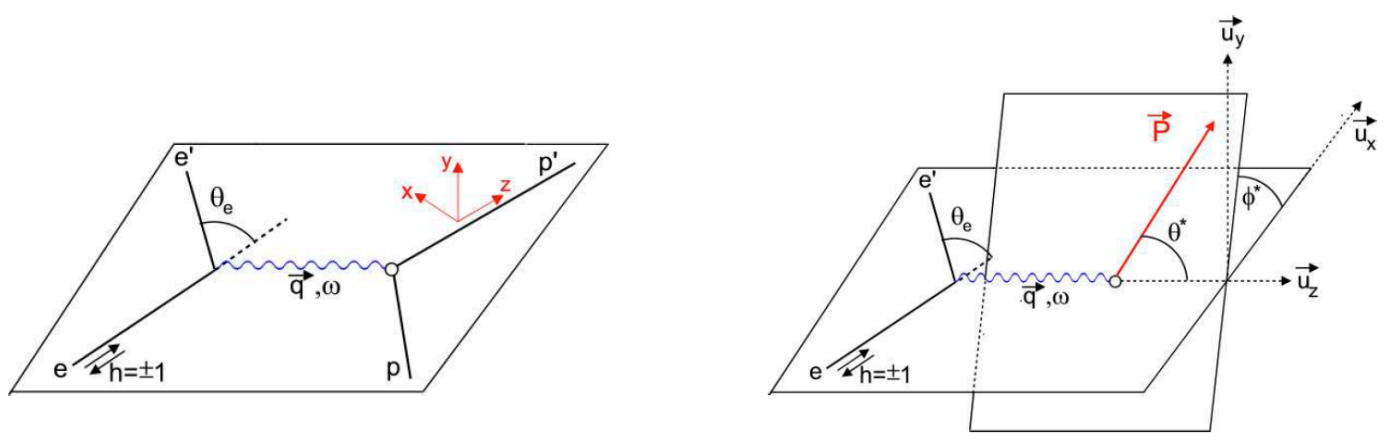

Figure 1.1: Kinematic variables in a transfer polarization experiment when the recoil polarization is measured(left) and when the target is polarized(Right) [1]

asymmetry is usually written as

$$
A_{P V}=\frac{N_{L}-N_{R}}{N_{L}+N_{R}}=P_{B} \frac{\sigma_{L}-\sigma_{R}}{\sigma_{L}+\sigma R}
$$

The asymmetries in PVeS experiments are much smaller than parity conserving experiments, at the part per million (ppm) or parts per billion (ppb) level. Furthermore, since only the beam is polarized, the precision to which we know the beam polarization is often the largest systematic error in these experiments. Parity violating electron scattering experiments can be designed to further our understanding of either the structure of the nucleon or fundamental interactions such as the parity violating weak force. To follow is a brief review of past experiments which relied on polarization measurements or polarimetry. Table 1.1 list these experiments and demonstrates the increasing demand on precision polarimetry. 
Table 1.1: Example of experiments relying on polarimetry

\begin{tabular}{|c|c|c|c|}
\hline Experiment & Beam Energy & Polarization & Polarimetry Precision \\
\hline$\overline{\text { SLAC }} 122$ PV-DIS (1978) ${ }^{[19]}$ & $16-22 \mathrm{GeV}$ & $37 \%$ & $6 \%$ \\
\hline Bates SAMPLE $(2000)^{[20]}$ & $0.2 \mathrm{GeV}$ & $39 \%$ & $4 \%$ \\
\hline JLab GEp/Gmp (1999) ${ }^{[21]}$ & $1-4 \mathrm{GeV}$ & $60 \%$ & $3 \%$ \\
\hline HERMES g1n DIS (2007) ${ }^{[22]}$ & $30 \mathrm{GeV}$ & $55 \%$ & $2.49 \%$ \\
\hline SLAC E154 DIS g1n (1997) ${ }^{[23]}$ & $48 \mathrm{GeV}$ & $82 \%$ & $2.4 \%$ \\
\hline MAMI PV-A4 (2004) ${ }^{[24]}$ & $0.85 \mathrm{GeV}$ & $80 \%$ & $2.1 \%$ \\
\hline JLab G0 $(2005)^{[25,26]}$ & $3.0 \mathrm{GeV}$ & $74 \%$ & $1.0 \%$ \\
\hline JLab Qweak (2017) ${ }^{[27]}$ & $1.2 \mathrm{GeV}$ & $88 \%$ & $0.62 \%$ \\
\hline $\operatorname{SLD} A_{L R}(2000)^{[28]}$ & $46.5 \mathrm{GeV}$ & $75 \%$ & $0.5 \%$ \\
\hline
\end{tabular}

\subsection{Nuclear Structure in Parity Conserving Reactions}

Understanding the structure of the nucleon is of fundamental importance in nuclear and particle physics; ultimately such an understanding is necessary to describe the strong force [21]. The parity conserving electromagnetic interaction provides us with an excellent probe to map out the structure of the nucleon while QCD calculations gives us a theoretical model that we may compare experimental results with. Experimental we measure cross sections at different angles, energies and momentum transfer squared, and from these measurements we are able to calculate the elastic electric and magnetic form factors. These form factors are related to the spatial charge and current distributions in the nucleon. The cross section for elastic scattering of an electron from a nucleon in the notation preferred today is [1]

$$
\frac{d \sigma}{d \Omega}=\left(\frac{d \sigma}{d \Omega}\right)_{M o t t} \times\left[G_{E}^{2}+\frac{\tau}{\varepsilon} G_{M}^{2}\right] /(1+\tau)
$$

where $\left(\frac{d \sigma}{d \Omega}\right)_{M o t t}$ is the Mott cross section, $G_{E(M)}$ is the electric(magnetic) Sachs Form Factor $(\mathrm{FF}), \tau=Q^{2} / 4 M^{2}, \varepsilon=\left[1+2(1+\tau) \tan ^{2} \frac{\theta_{e}}{2}\right]^{-1}$ is the virtual photon polarization, $M$ is the mass of the nucleon, and $Q^{2}$ is the square of the energy-momentum transfer. Rosenbluth separation has been an extremely powerful method for extracting the individual form factors. By measuring and plotting the elastic cross section at different values of $\varepsilon$ and using Equation 1.3 the form factors are given by slope $=\frac{1}{\tau} G_{E p}^{2}$ and intercept $=G_{M p}$. However at higher values of $Q^{2}$, Rosenbluth separation becomes more difficult as the $\tau G_{M p}$ dominates 
as $\tau$ is increased. Polarization transfer methods are an alternative to measuring the form factors at higher values of $Q^{2}$. Figure 1.2 shows an example of a Rosenbluth separation (left) analysis and a transfer polarization experiment(right).
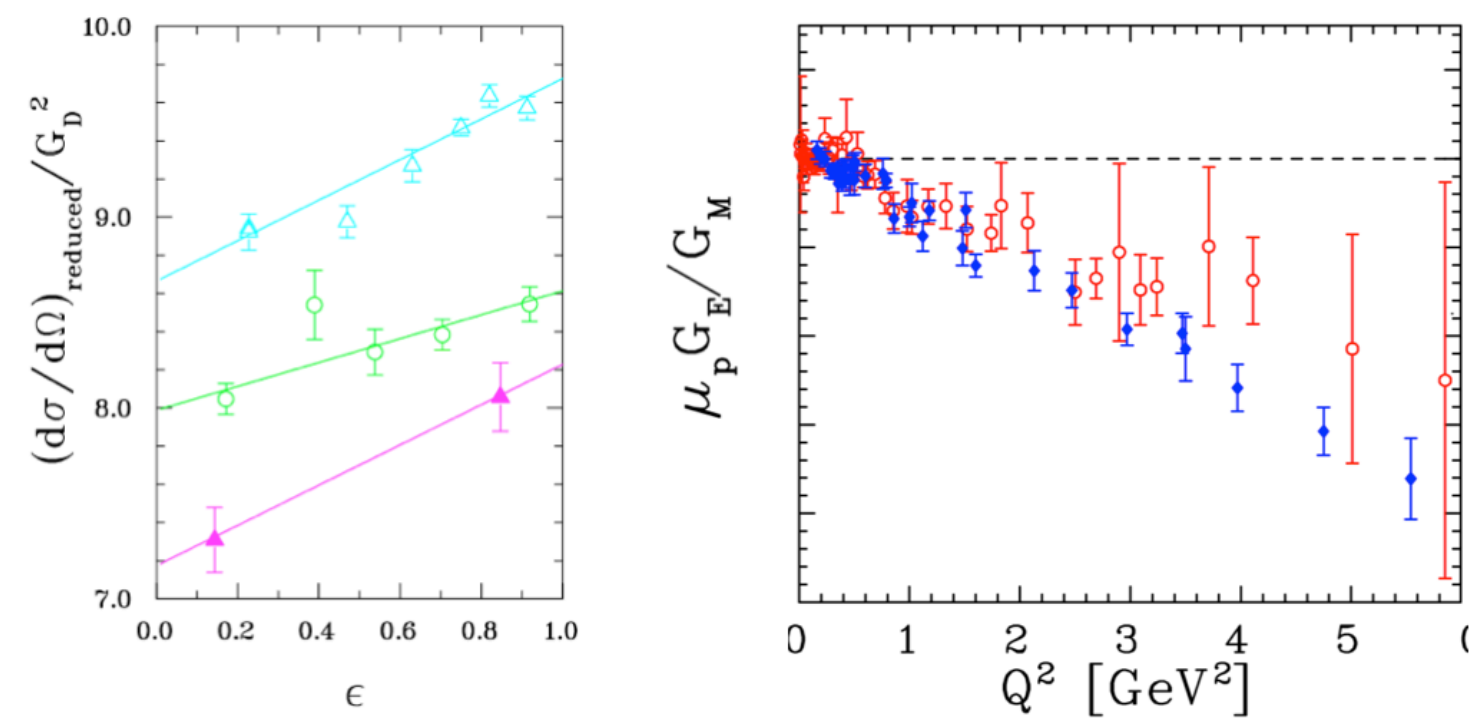

Figure 1.2: (Left) Demonstration of Rosenbluth separation at $Q^{2}$ values of 2.5 (open triangle), 5.0 (circles), 7.0 (filled triangles) $\mathrm{GeV}^{2}$ [2] (Right) Comparison of the ratio of $G_{E p} / G_{M p}$ by using Rosenbluth separation and polarization transfer data.[1]

The $G_{E p} / G_{M p}$ ratio by polarization transfer in $\vec{e} e \rightarrow p \vec{p}$ experiment was performed in Hall A at JLab in 1999 and is an example of using polarization transfer to obtain nucleon form factors. Here an incident polarized electron $(\sim 1$ to $4 \mathrm{GeV})$ was elastically scattered from an unpolarized proton (liquid hydrogen target) and the polarization was transferred to the scattered proton. A focal plane polarimeter was installed in the hadron high resolution spectrometer (HRS) which enabled the transverse polarization, $P_{t}$, and the longitudinal polarization, $P_{l}$, of the scattered proton to be measured. The ratio of the electric to the magnetic form factors, $G_{E p}$ and $G_{M p}$ respectively, was calculated using the relation [21]

$$
\frac{G_{E p}}{G_{M p}}=\frac{-P_{t}}{P_{l}} \frac{E_{e}+E_{e^{\prime}}}{2 M} \tan \frac{\theta_{e}}{2}
$$

Where $E_{e\left({ }^{\prime}\right)}$ is the energy of the incident (scattered) electron, $\theta_{e}$ is the scattering angle measured with respect to the incident electron, and $M$ is the mass of the proton. This powerful technique of polarization transfer demonstrated for the first time that the $Q^{2}$ dependence of 
$G_{E p}$ and $G_{M p}$ is significantly different and that there was a definite difference in the spatial distribution of charge and magnetization currents in the proton [21].

Using a polarized target in polarized electron scattering allows for a new class of deep inelastic scattering (DIS) experiments that are designed to study the spin structure of the nucleon. Two examples of this are the E154 experiment at SLAC and HERMES at DESY. Experiment E154, The Precision Determination of the Neutron Spin Structure Function $g_{1}^{n}$, used a polarized electron beam and a polarized Helium-3 target to learn about the internal spin structure of the neutron [23] by measuring the form factor $g_{1}^{n}$. The HERMES collaboration measured the spin structure function of the proton and deuteron and therefore was also able to deduce it for the neutron. This experiment used polarized positrons accelerated to energies of $27.6 \mathrm{GeV}$ [22] incident upon a polarized gas target. Both of these experiments were part of the ongoing effort for a detailed investigation of the spin structure of the nucleon and the determination of the partonic contribution of its spin projection. One of the outcomes of these experiment was that we still didn't have a firm grasp of what contributes to the spin of the nucleon, because it seems only a small fraction of the total spin of the nucleon could be accounted for from the valence quarks. If the contribution is due to sea quarks then further experiments would have to be considered at higher energies.

\subsection{Parity Violation for Fundamental Interactions}

In 1978 the Weinberg-Salam model, which unified the electromagnetic and weak forces, was tested at the Stanford Linear Accelerator (SLAC) by experiment E122[3][19]. This marked the first measurement of parity violation in the neutral weak current and validated electroweak theory. The experiment featured the scattering of $\sim 20 \mathrm{GeV}$ polarized electrons from an unpolarized deuterium target. The measured asymmetry is shown in Figure 1.3. The success of E122 has lead to a whole new generation of parity-violating electron scattering experiments using polarized beams.

The SLD experiment at SLAC measured the Z-boson-lepton coupling asymmetry parameters, $A_{e}, A_{m}$ and $A_{t}$ and therefore also the effective weak mixing angle, $\theta_{\text {Weak }}^{\text {eff }}$ [28]. Z-bosons were created by $e^{+} e^{-}$annihilation and subsequently decayed into $e^{+} e^{-}, \mu^{+} \mu^{-}$, or $\tau^{+} \tau^{-}$pairs that were detected. The results were consistent with the Standard Model which assumes lepton universality and that the measured asymmetry should be the same for all three lepton flavors. The effective weak mixing angle could be calculated by the relation 


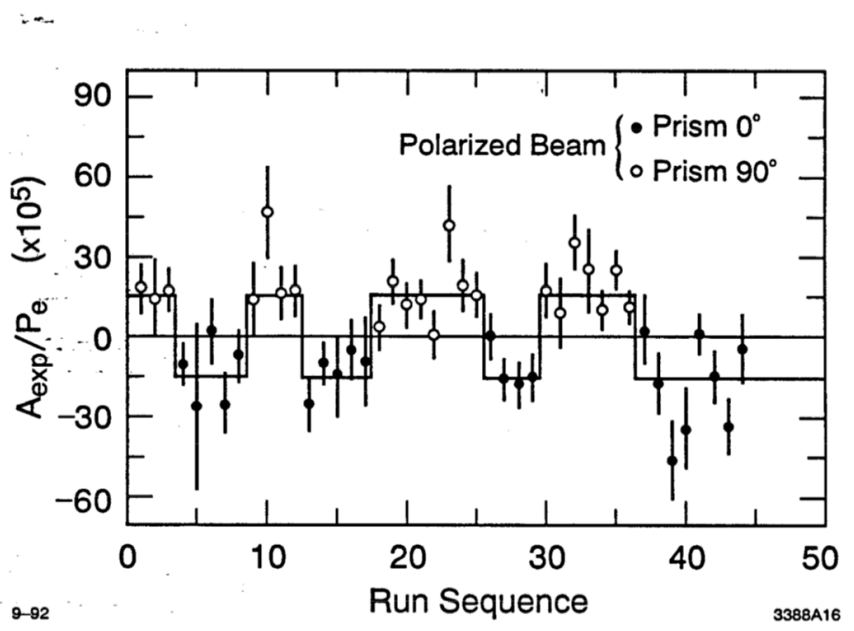

Figure 1.3: Measured asymmetry in E122. Each run was 1 to 3 hours and the solid line is the expected result [3]

$$
A_{l}=\frac{2\left(1-4 \sin ^{2} \theta_{\mathrm{Weak}}^{\text {eff }}\right)}{1+\left(1-4 \sin ^{2} \theta_{\text {Weak }}^{\text {eff }}\right)}
$$

The QWEAK experiment in Hall C at JLab performed the most accurate measurement of the weak charge of the proton by measuring the parity-violating elastic asymmetry of longitudinally polarized electrons from a liquid hydrogen target at small momentum transfer. It is the smallest and most precise asymmetry measurement of $\vec{e} p$ scattering to date and set many milestones at JLab. The asymmetry measured was remarkably small at 279 parts per billion (ppb). The helicity of the $1.16 \mathrm{GeV}$ beam was flipped 960 times per second in order to control systematics associated with helicity correlated asymmetries. Such a precise measurement on the weak charge of the proton also provided a high precision measurement of the weak mixing angle $\sin \theta_{W}^{2} .[7,27,29]$

\subsection{Parity Violation for Nucleon Structure}

Parity violating electron scattering experiments can also provide information about nucleon structure. An important example is the SAMPLE (Single Anomalous Moment of the Proton by Longitudinal Electron Scattering) experiment at the MIT-Bates Laboratory which was designed to determine the role of the quark and anti-quark sea in the nucleon[20]. This experiment, for which the Bonner Prize was rewarded to Bob McKe- 
own in 2009, ushered in the modern era of PV experiments. It focused on strange quark contributions to the proton spin. A polarized electron beam was used incident upon a liquid hydrogen target and an asymmetry on the order of 0-20 ppm was measured. As a second example we can look at the PV-A4 experiment. The results from the PV-A4 experiment at the Mainzer Mikrotron (MAMI) were published in 2004 and it was the first PV experiment to count individual scattering events. The experiment was designed as being complimentary to the SAMPLE and the HAPPEX (Jlab Hall A) experiments and aimed to provide a direct measurement of the strangeness contribution to the vector form factor of the proton. This is important because it had been suggested that the strange quark sea could contribute to $\approx 50 \%$ of the nucleon mass and $\approx 10 \%$ to the overall nucleon spin [24]. experiment at the MIT-Bates Laboratory which was designed to determine the role of the quark and antiquark sea in the nucleon[20]. This experiment, for which the Bonner Prize was rewarded to Bob McKeown, ushered in the modern era of PV experiments. It focused on strange quark contributions to the proton spin. A polarized electron beam was used incident upon a liquid hydrogen target and an asymmetry on the order of 0-20 ppm was measured. As a second example we can look at the PV-A4 experiment. The results from the PV-A4 experiment at the Mainzer Mikrotron (MAMI) were published in 2004 and it was the first PV experiment to count individual scattering events. The experiment was designed as being complimentary to the SAMPLE and the HAPPEX (Jlab Hall A) experiments and aimed to provide a direct measurement of the strangeness contribution to the vector form factor of the proton. This is important because it had been suggested that the strange quark sea could contribute to $\approx 50 \%$ of the nucleon mass and $\approx 10 \%$ to the overall nucleon spin [24]. experiment at the MIT-Bates Laboratory which was designed to determine the role of the quark and antiquark sea in the nucleon[20]. This experiment, for which the Bonner Prize was rewarded to Bob McKeown, ushered in the modern era of PV experiments. It focused on strange quark contributions to the proton spin. A polarized electron beam was used incident upon a liquid hydrogen target and an asymmetry on the order of 0-20 ppm was measured. As a second example we can look at the PV-A4 experiment. The results from the PV-A4 experiment at the Mainzer Mikrotron (MAMI) were published in 2004 and it was the first PV experiment to count individual scattering events. The experiment was designed as being complimentary to the SAMPLE and the HAPPEX (Jlab Hall A) experiments and aimed to provide a direct measurement of the strangeness contribution to the vector form factor of the proton. This is important because it had been suggested that the strange quark sea could contribute to $\approx 50 \%$ of the nucleon mass and $\approx 10 \%$ to the overall nucleon spin [24]. 
The G0 experiment in Hall $\mathrm{C}$ at JLab also measured the strange quark contribution to the parity violating asymmetry and required polarimetry at the $1 \%$ level $[25,26]$. The precision to which the beam polarization was known was a key systematic error in all of these experiments. From Table 1.1 we can see most of them had percent level precision, with Qweak and SLD being the only two that required $<1 \%$ precision. The future of PVeS will depend on high precision polarimetry, in particular the program in Hall A at Jefferson Laboratory.

\subsection{Upcoming experiments in Hall A at Jefferson Labora- tory}

The upcoming PVeS experiments in Hall A at Jefferson Laboratory has increasingly stringent demands on polarimetry requirements. The Møller polarimeter, which is the focus of this dissertation, is an instrumental apparatus for the success of the physics program in Hall A. The upcoming experiments include CREX, PREX-II, SoLID and MOLLER. CREX and PREX are scheduled to start in 2019 and will require polarimetry at the $<1 \%$ level while SoLID and MOLLER will require $<0.5 \%$ level precision, which would make it the most precise electron beam polarization measurement to date.

CREX and PREX-II aim to measure the weak charge by exploiting the fact that the Z-boson of the weak interaction primarily couples to neutrons. By using the neutron rich and "doubly magic" nuclei of $P b^{208}$ and $C a^{48}$ these experiments will be measuring an $A_{P V}$ of 2.2ppm for CREX (Ca Radius EXperiment) and $0.6 \mathrm{ppm}$ for PREX (Pb Radius EXperiment). The asymmetry measurement will provide a model independent probe of neutron densities unlike the present knowledge of the neutron distributions which comes primarily from model dependent hadron scattering [30]. The parity violating asymmetry, $A_{P V}$, in the Born approximation is

$$
A_{P V} \approx \frac{G_{F} Q^{2}}{4 \pi \alpha \sqrt{2}} \frac{F_{W}\left(Q^{2}\right)}{F_{C h}\left(Q^{2}\right)}
$$

Where $G_{F}$ is Fermi's constant, $\alpha$ is the fine structure constant, $F_{C H}\left(Q^{2}\right)$ is the Fourier transform (FT) of the charge density and $F_{W}\left(Q^{2}\right)$ is the FT of the weak form factor. $F_{W}\left(Q^{2}\right)$ is related to the weak charge density, $\rho_{W}(r)$ by

$$
F_{W}\left(Q^{2}\right)=\frac{1}{Q_{W}} \int d^{3} r \frac{\sin Q r}{Q r} \rho_{W}(r)
$$




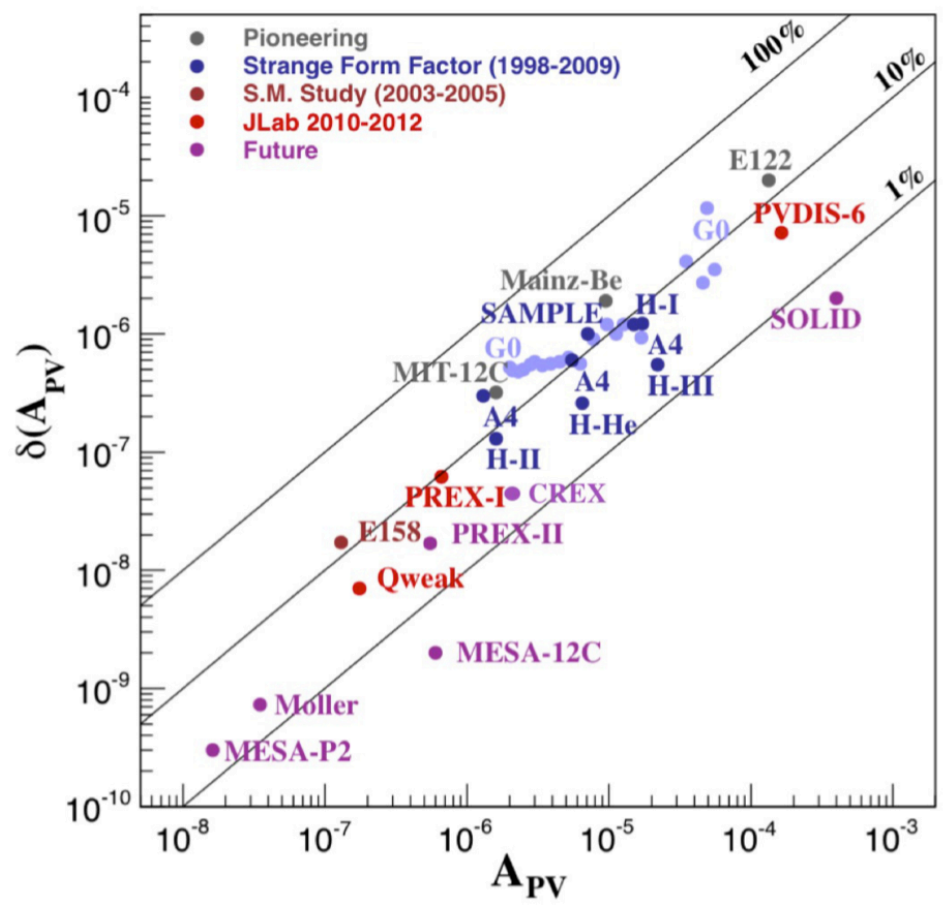

Figure 1.4: Upcoming experiments in Hall A (CREX, PREX-II, MOLLER, SoLID) will be pushing the frontier by measuring extremely small asymmetries with remarkable precision (Figure courtesy of Kent Paschke) 
where it is normalized so that $F(Q=0)=1$. The total weak charge of the nucleus is $Q_{W}=\int d^{3} r \rho_{W}(r)[31] . A_{P V}$ is also related to the neutron density by

$$
A_{P V} \approx \frac{G_{F} Q^{2}}{4 \pi \alpha \sqrt{2}}\left[1-4 \sin ^{2} \theta_{W}-\frac{F_{n}\left(Q^{2}\right)}{F_{C h}\left(Q^{2}\right)}\right]
$$

where $F_{n}\left(Q^{2}\right)$ is the FT of the neutron density.

So by measuring $A_{P V}$ we are able to find the weak form factor which can then give us the radius of the neutron distribution in the nucleus, $R_{n}$. The neutron skin $\left(R_{n}-R_{p}\right)$ can then be found which provides unique input into such diverse areas such as neutron star structure, heavy ion collisions, atomic parity violation, the isovector sector of nuclear theories, new coupled cluster calculations sensitive to three neutron forces, and a critical bridge between ab-initio approaches and density functional theories (DFT)[31].

CREX and PREX-II, which will require $0.8 \%$ and $2.2 \%$ polarimetry, will be followed by the MOLLER experiment and the SoLID program which will both require $0.4 \%$ polarimetry. The MOLLER (Measurement of a Lepton Lepton Electroweak Reaction) experiment will provide the most precise measurement to date of the weak mixing angle, a factor of 5 times better than the E158 result. The prediction for $A_{P V}$ for the proposed experimental design is 33 parts per billion (ppb) and the goal is to measure this quantity with an overall precision of $0.7 \mathrm{ppb}$ and thus achieve a $2.4 \%$ measurement of $Q_{W}^{e}[17,32]$. The SoLID (Solenoidal Lare Angle Intensity Device) program at Jlab will allow for a whole new class of parity violating deep inelastic scattering experiments (PVDIS). Some of the physics issues these experiments will address are to search for new interactions beyond the Standard Model (SM) in a unique way, to search for Charge Symmetry violation (CSV) at the quark level, to search for higher-twist effects in $A_{P V}$, to measure the $d / u$ ratio in the proton (without requiring any nuclear corrections), and to determine if additional CSV is induced in heavier nuclei[33].

The common theme to all the experiments described in that last four sections are that they relied on precision polarimetry. The progress of polarized electron scattering experiments is dependent upon further improving the precision of beam polarization measurements and the motivation for this dissertation. In Chapter 2, I will briefly review the different type of polarimetry techniques used at electron accelerator facilities, with an emphasis on Jefferson Laboratory (JLab). The focus of this dissertation is on Møller Polarimetry and in particular the newly upgraded polarimeter in Hall A at JLab. Chapter 3 will describe the apparatus and how it is modeled in Monte Carlo simulations. A systematic study of the 
uncertainties will be presented in Chapter 4, and recent results from the DVCS experiment and the 2019 commissioning data will be presented in Chapter 5. In preparations for future polarimetry demands, the plan for CREX and PREX running will be presented in Chapter 6 along with recommendations to achieve $0.4 \%$ precision required for the MOLLER experiment and SoLID program on Hall A. 


\section{CHAPTER 2}

\section{PRECISION ELECTRON BEAM POLARIMETRY TECHNIQUES}

There are three different types of polarimeters typically used at electron beam accelerator facilities: Mott, Compton, and Møller polarimeters, each with their own advantages and disadvantages. Mott polarimeters are limited to $\mathrm{MeV}$ range energy scales so they are typically found at the low energy region of electron accelerators, called the "injector region" at $\mathrm{Jlab}$, and may not accurately represent the polarization that is being delivered to the main experiment after the electrons are accelerated to higher energies. Compton polarimeters are only practical at high current but have the advantage of being "non-invasive" so they may operate without affecting the main experiment. Møller polarimeters can operate at a wide range of energies but are limited to low beam currents $(<2 \mu \mathrm{m})$. They are also invasive so experimental data acquisition must stop in order to perform a polarization measurement. In the following sections these three basic types of polarimetry will be discussed.

Before describing the different types of polarimetry, let me briefly explain how a polarized electron beam is produced. Almost all accelerators now use photoemission from a GaAs or a related material because this method allows for both peak high currents and rapid polarization reversal. The operation and two fundamental aspects of this type of source is nicely summarized in "Polarized electron sources: The next generation" by Larry Cardman [34]:

1) the polarized electrons are produced by optically pumping electrons from the top of the valence band to the bottom of the conduction band of a suitable direct-bandgap semiconductor (usually GaAs); and 2) these electrons are able to escape from the semiconductor because its surface has been atomically 
cleaned and then covered with a monolayer of an alkali metal (typically Cs) and oxidized (most often using NF3) to lower the work function of the surface to the point where the energy of the electron in the vacuum outside the material is lower than its energy in the bulk material, a condition referred to as negative electron affinity (NEA). A typical GaAs surface that has been treated in this manner will have a quantum efficiency of order a few percent. One watt of laser power will produce $6.5 \mathrm{~mA}$ of beam current from a GaAs photocathode having a $1 \%$ quantum efficiency, so very reasonable laser power can provide very high electron currents.

Photoemission from GaAs produces a beam polarization of $40-50 \%$ as seen in Figure 2.12. Shortly thereafter it was discovered that using a strained GaAs lattice could provide even a higher polarization of around $86 \%$ [34]. The reason for the higher polarization is that the uniaxial strain to a crystal of GaAs removes the band degeneracy by breaking the symmetry of the crystalline structure[35]. This technique for production of a polarized electron beam has been used at JLab since 2004 [36].

\subsection{Mott Polarimetry}

Mott polarimeters, as the name suggests, use Mott scattering to measure the electron beam's transverse polarization by scattering electrons off a high $\mathrm{Z}$ nucleus. The single spin asymmetry is measured and converted into a beam polarization by combining it with the theoretical Sherman functions that determine the analyzing power. The analyzing power is large at $\mathrm{MeV}$-scale energies and for this reason Mott polarimeters are found at the injector region of an electron accelerator, before the beam is accelerated to high $(\mathrm{GeV})$ energies. The relationship between the measured asymmetry, $\varepsilon$, the transverse beam polarization $P_{T}$ and the Sherman function $S(\theta)$ is

$$
\varepsilon=P_{T} S(\theta)
$$

The Sherman function is a theoretically calculated value for single-atom scattering and depends on the electron's kinetic energy, the atomic number of the target nucleus, and the scattering angle of the electron. [6]. The precision to which the Sherman function is known has been improved over the years by using more accurate models of the electronic and nuclear charge distributions which now include the finite size of the nucleus and the 
latest nuclear and atomic structure data. The largest uncertainties associated with the Sherman function are due to radiative corrections and experimental evidence suggest that their contribution is considerably smaller than $1 \%$. [5, 37]

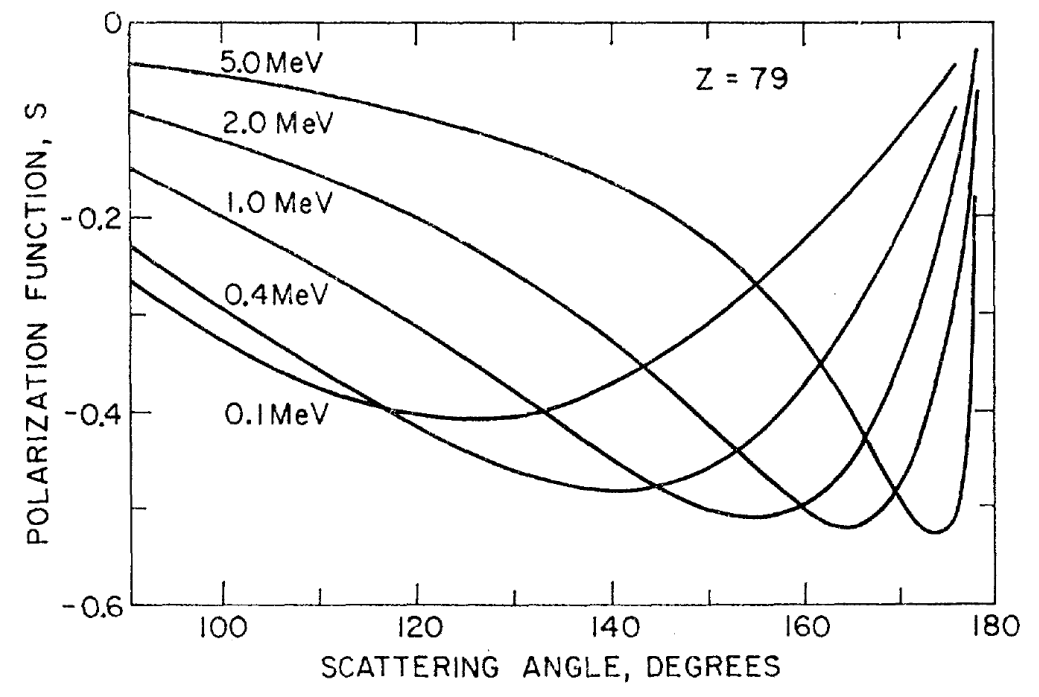

Figure 2.1: Sherman Functions for Gold nucleus. These function show the asymmetry in a Coulomb field as a function of scattering angle when assuming a point like nucleus and no screening. [4]

One of the biggest challenges with Mott polarimeters is overcoming the large background from multiple scattering. This is handled by taking measurements with different thickness targets and extrapolating the measured asymmetries to a zero thickness target where the theoretical Sherman function described above is valid. Once the dependence of target thickness is known an effective Sherman function is used to calculate the polarization for the different foil thicknesses. An example of this treatment is shown in Fig. 2.2

The Mott polarimeter at Jefferson Lab [38] is optimized to run at a beam energy of $\sim 5 \mathrm{MeV}$. A target ladder with $14 \mathrm{Au}, \mathrm{Ag}$ or $\mathrm{Cu}$ foils of varying thicknesses are used as targets. Four detectors are used to measure the horizontal and vertical transverse polarizations. The setup is shown in Fig. 2.3. The electron beam comes in from the right and scatters from one of the foil targets. The detectors are arranged to detect electrons which scatter at $172.7^{\circ}$. An asymmetry is constructed by measuring the rates while flipping the helicity of the incident beam. The combined systematic errors on polarization measurements has been reported to be $1.1 \%$ [5]. 


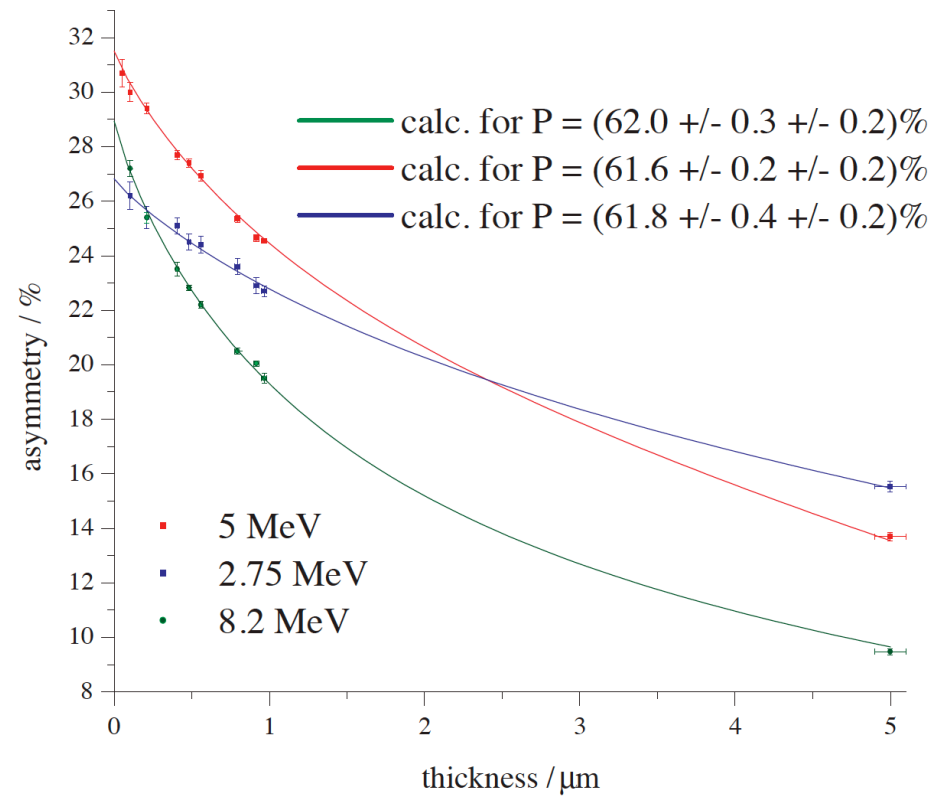

Figure 2.2: Foil - thickness extrapolation for $2.75,5$ and $8.2 \mathrm{MeV}$. Lines symbolize calculation and dots the measurement. [5]

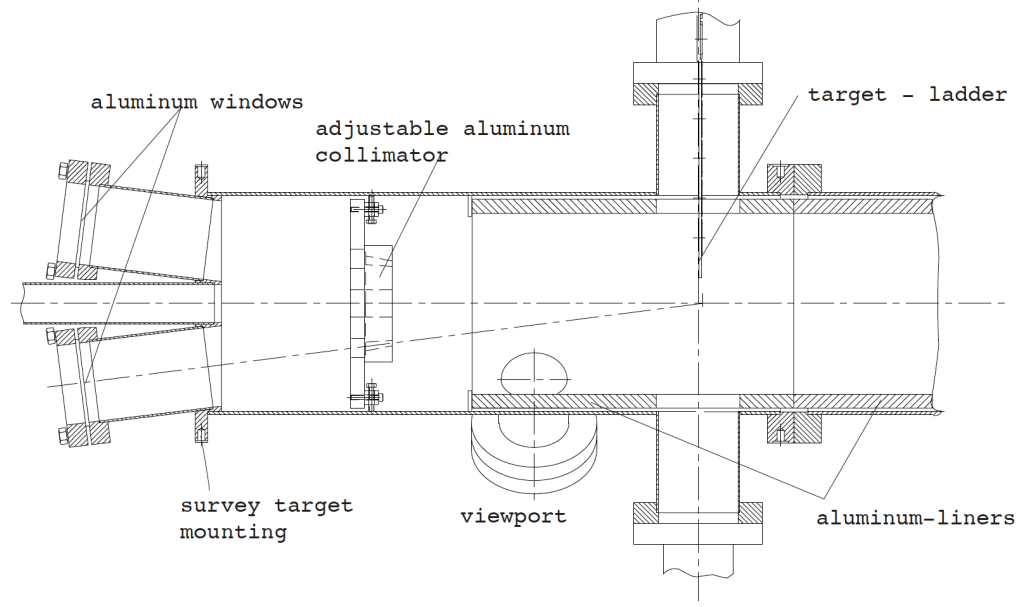

Figure 2.3: MeV Mott Polarimeter at Jefferson Lab. Electron is incident from right, scatters off target foil, and is detected after passing through the aluminum windows 
Even though we have a fairly well known polarization measurement at the electron source that does not necessarily translate to knowing the polarization of the beam where the main experiments are taking place. After leaving the injector site, the electrons are accelerated and bent around the racetrack design of the accelerator. As the electrons are bent their spins are rotated as a result of spin precession [39]. Therefore it is important to have additional Compton or Møller polarimeters located in the experimental halls.

\subsection{Compton Polarimetry}

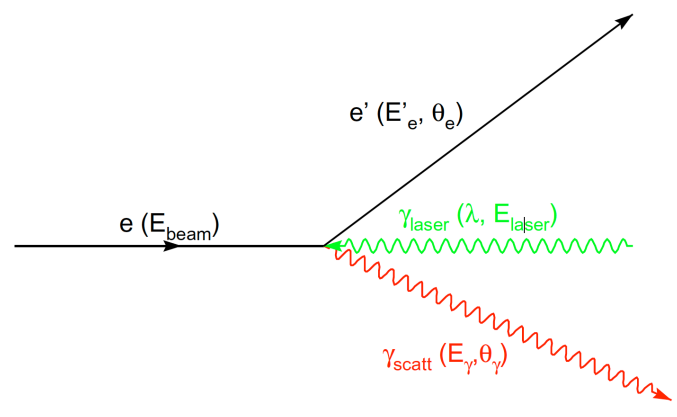

Figure 2.4: Compton Scattering [6]

Compton scattering can be used as another powerful polarimetry method. A Compton polarimeter scatters a circularly polarized photon from a laser off an electron. The backscattered photon and/or scattered electron is detected and an asymmetry in scattering rates is measured by flipping the helicity of the primary beam. One large advantage of a Compton polarimeter is that it is non-invasive to the main experiment which allows polarization measurements to be performed while the experiment is running. Since they are non-invasive they are often the only choice of polarimetry at storage rings and colliders facilities. Unlike Mott polarimeters, Compton polarimeters can be located in the experimental hall so spin precession in the arcs does not have to be taken into account. Compton polarimeters also have an advantage over Moller polarimeters because they are able to operate at high current $(\sim 100 \mathrm{muA}$ at Jlab). Primary disadvantages are they are not reliable at low currents $>1 \mu \mathrm{A}$, the analyzing power is small at the GeV level (see Figure 2.5) and at higher beam energies large synchrotron radiation can lead to high backgrounds. 
Figure 2.4 depicts the process of scattering a circularly polarized photon off a longitudinally polarized electron. The energy of the scattered photon $E_{\gamma}$ is

$$
E_{\gamma} \approx E_{\text {laser }} \frac{4 a \gamma^{2}}{1+a \theta_{\gamma}^{2} \gamma^{2}}
$$

where $\gamma=E_{e} / m_{e}, \theta_{\gamma}$ is the angle of the scattered photon relative to the incident electron, and $a$ is defined as

$$
a=\frac{1}{1+4 \gamma E_{\text {laser }} / m_{e}}
$$

It is useful to define the quantity $\rho=E_{\gamma} / E_{\gamma}^{\max }$ where $E_{\gamma}^{\max }=4 a E_{\text {laser }} \gamma^{2}$. Then we can write the unpolarized differential cross section as

$$
\frac{d \sigma}{d \rho}=2 \pi r_{0}^{2} a\left[\frac{\rho^{2}(1-a)^{2}}{1-p(1-a)}+1+\left(\frac{1-p(1+a)}{1-p(1-a)}\right)^{2}\right]
$$

where $r_{0}$ is the classical electron radius. The analyzing power for circularly polarized photons and longitudinal polarized electrons is

$$
A_{\text {long }}=\frac{\sigma^{++}-\sigma^{-+}}{\sigma^{++}+\sigma^{-+}}=\frac{2 \pi r_{0}^{2} a}{(d \sigma / d \rho)}(1-\rho(1+a))\left[1-\frac{1}{1-\rho(1-a))^{2}}\right]
$$

Figure 2.5 shows the unpolarized differential cross section and longitudinal analyzing power for three different beam energies and also how the analyzing power is strongly dependent on both the beam energy and the energy of the scatter photon.

The main elements of the Compton polarimeter in Hall A is shown in figure 2.6. The first two dipoles bend the main electron beam to the interaction region of the polarimeter. Here a $\sim 532 \mathrm{~nm}$ laser is coupled to a Fabry-Perot optical cavity which allows several $\mathrm{kW}$ of laser power to be stored which is necessary to obtain the required rates for the currents available at Jefferson Lab. The main beam crosses at a very small angle in the center of the cavity. The back scattered photon is detected as a gamma ray in the photon detector. Depending on the energy of the photon either a GSO (low energy) or $\mathrm{PbWO}_{4}$ crystal is used to produce scintillation light that can be detected by a PMT. The scattered electron is bent by a third dipole to a segmented silicon strip detector. The third dipole momentum analyzes the scattered electrons allowing their energies to be obtained from their detected positions in the silicon detector. The main electron beam is returned to the beam line by the third and fourth dipoles and continues along to the main experimental target. 

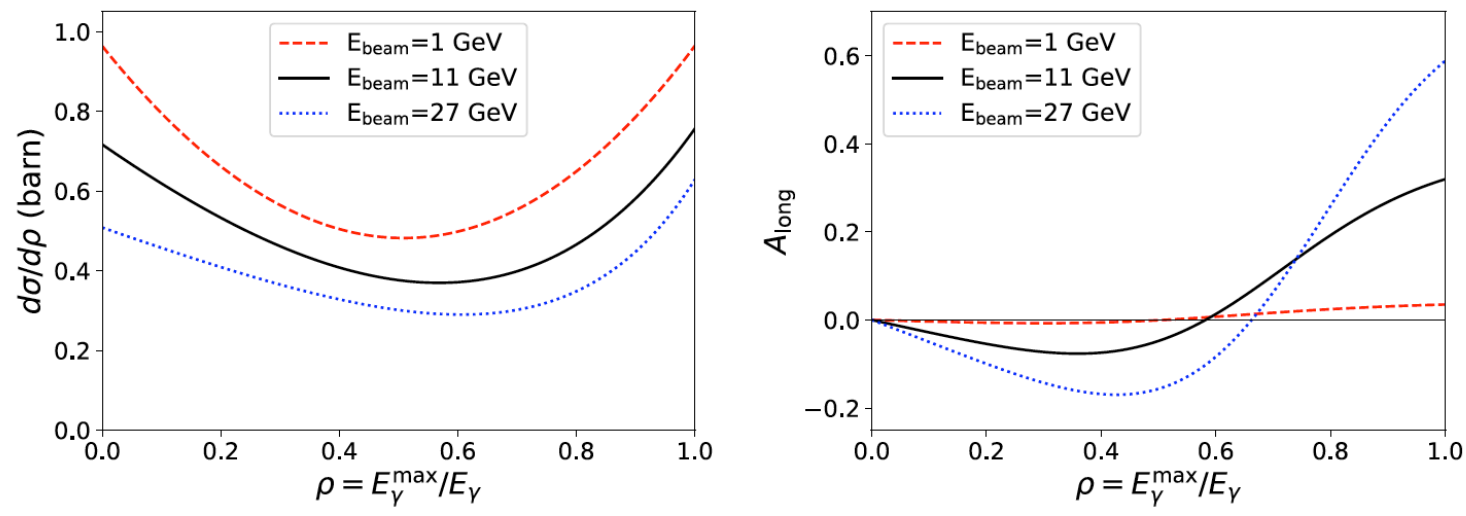

Figure 2.5: Compton Scattering Cross Section (left) and longitudinal analyzing power (right) assuming a $532 \mathrm{~nm}$ laser colliding with an electron beam at $1 \mathrm{GeV}, 11 \mathrm{GeV}$ and $27 \mathrm{GeV}$.[6]

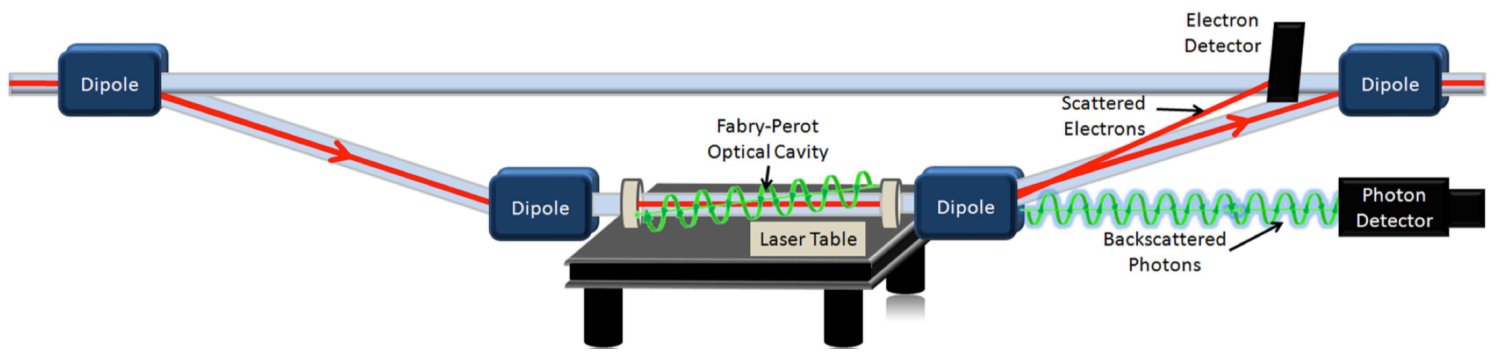

Figure 2.6: Compton Polarimeter in Hall A [7] 
The photon and electron detectors can be used to provide somewhat independent measurements of the beam polarization. Also, the electron detector can be used in coincidence with the photon detector to reduce backgrounds in the photon detector. If the polarimeter is operated in "counting mode" then for each helicity cycle an asymmetry vs. energy spectrum can be constructed and this result is then fit to the theoretical analyzing power and the polarization is extracted. An example of this analysis is shown in figure 2.7.
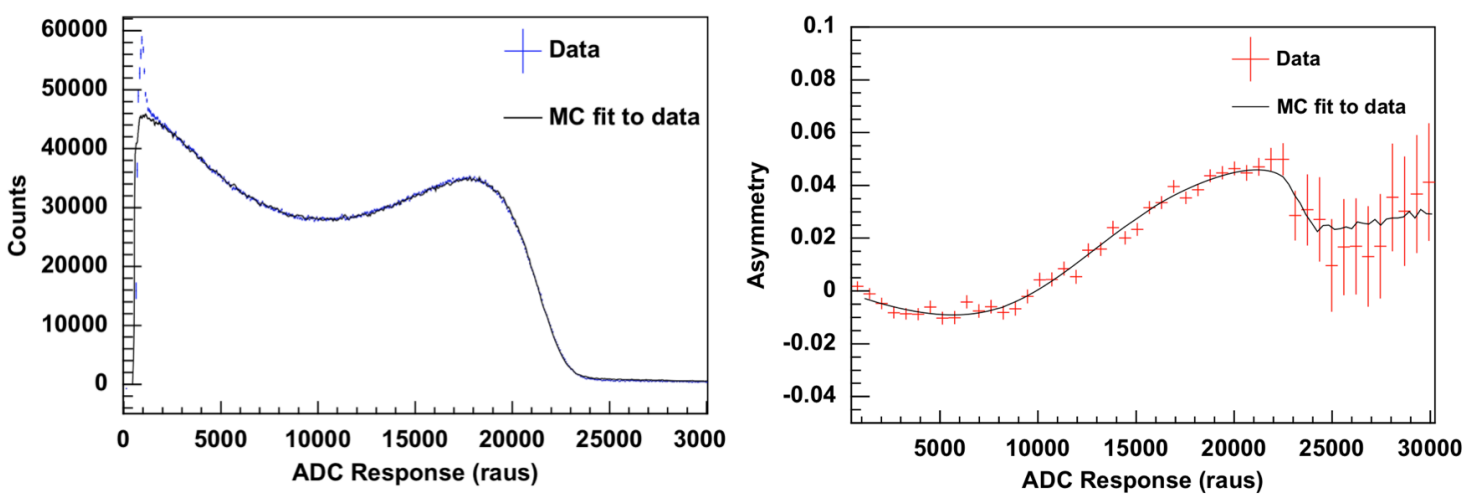

Figure 2.7: (Left)Compton photon energy spectrum (Right)Asymmetry vs photon energy spectrum. Both plots show data fit to Monte Carlo simualtion [8]

If the polarimeter is operated in "integrating mode", an energy weighted asymmetry is constructed by integrating the total energy deposited in the photon detector for each helicity state. This has the advantage of measuring a larger asymmetry since the analyzing power is largest at the higher end of the scattered photon energy spectrum. An example of this analysis is shown in figure 2.8. The results shown are from the HAPPEX-III experiment which ran in 2009. Here the systematic uncertainty was quoted as $0.94 \%$ for a beam energy of 3.4 $\mathrm{GeV}$ which was dominated by the uncertainty in the laser polarization. More recently, the Hall C Compton polarimeter reported a systematic error of $0.59 \%$ [40] at a beam energy of $1.16 \mathrm{GeV}$ used for the QWEAK experiment which ran from 2010 to 2012 . 


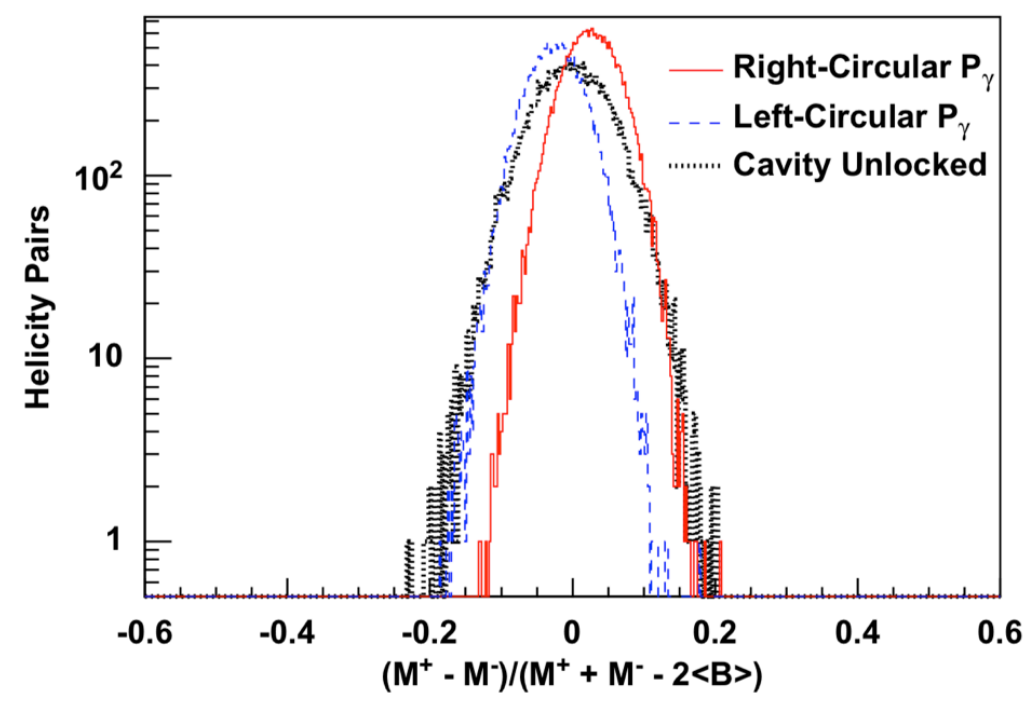

Figure 2.8: Integrating mode asymmetry for different circular polarization laser states and with the laser cavity unlocked (for background subtraction)Here $M^{+(-)}$is the measured integrated signal plus background for positive (negative) helicity electrons. [8]

\subsection{Møller Polarimetry with Polarized Foil Target}

Møller polarimeters measure the electron beam polarization by measuring the difference in Møller scattering rates off a polarized target as the helicity of the beam is flipped. Møller scattering is the elastic scattering of two electrons and since it is a pure QED process, its cross-section is calculated to very high precision[41]. Møller polarimeters have an advantage over Compton and Mott polarimeters because they can make measurements over a wide range of beam energies. Disadvantages include that they must be operated at low currents to avoid target depolarization and measurements are invasive so dedicated Møller runs must be taken. The dominant systematic error in Møller polarimeters is the precision to which the target polarization is known.

There are two different target polarization methods used in Møller polarimeters 2.9. The first approach is a tilted target where the foils are positioned in the beam line at an angle, typically at $\sim 20^{\circ}$. This allows a relatively low field $(\sim .02 T)$ to saturate or polarize the target "in plane". The holding field is typically reversed between data runs to eliminate systematic errors[42]. The polarization of the target is measured using pick-up coils and experiments have claimed target polarization uncertainties of $1.5 \%-3.0 \%$ relative[6]. The 
original Møller polarimeter in Hall A used this method which lead to overall precision on beam polarization measurements of 3\%[10]. With over a decade of experience and further improvements upon the tilted-foil apparatus an uncertainty of $1.7 \%$ was claimed[9]. The second method is the "brute-force" target which is the current design in Hall A. Here the foils are positioned in the beam line normal to the incoming beam. The foils are polarized out-of-plane using a 3-4T magnetic field and and knowledge of the target polarization relies on existing bulk measurements of the target material (typically iron). This method reduces the uncertainty of the target polarization. For the Hall A polarimeter, the target polarization uncertainty went from $1.5 \%$ for the tilted target to $0.35 \%$ using the "brute-force" method[9].
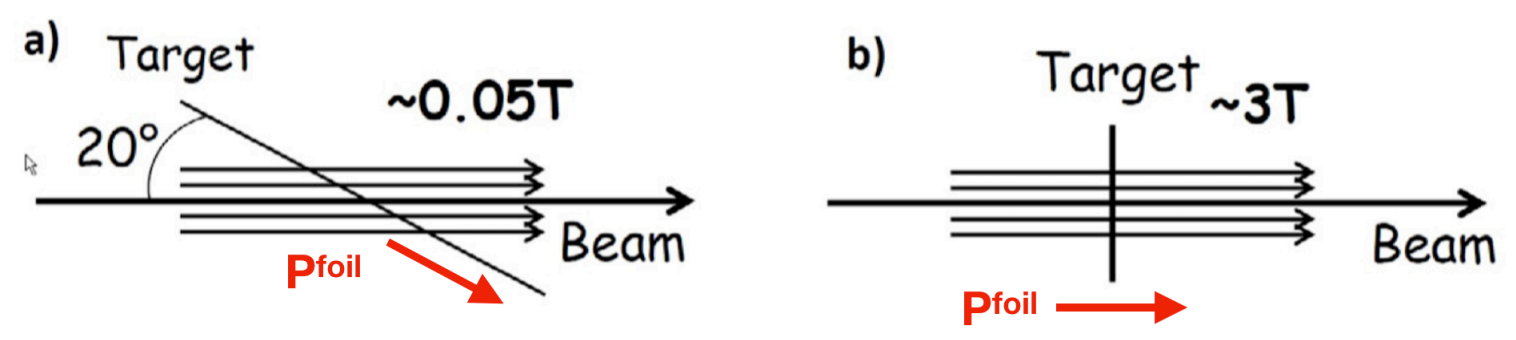

Figure 2.9: a) Scheme of "classic" technique of ferromagnetic foil polarization in the foil plane. b) Scheme of "brute force" technique of ferromagnetic foil polarization in out-of-thefoil plane.[9]

\subsubsection{Polarized Møller Scattering}

Electron-electron scattering, with arbitrary spin orientation for the beam and target, has been calculated in lowest order QED by many authors [43-46], and the basic formulas for (non parity-violating) polarized Møller scattering are given in many places. Following the convention in [6], the Møller cross-section in the center-of-mass (CM) frame is:

$$
\frac{d \sigma}{d \Omega^{*}}=\frac{d \sigma_{\circ}}{d \Omega^{*}}\left[1+\sum_{i, j=x, y, z} P_{i}^{\mathrm{b}} A_{i j} P_{\mathrm{j}}^{t}\right]
$$


where $P_{i}^{\mathrm{b}(\mathrm{t})}$ is the beam(target) polarization projected on the $i$-th axis, $A_{i j}$ is known as the analyzing power, and the unpolarized cross-section, $\frac{d \sigma_{\circ}}{d \Omega^{*}}$, is

$$
\frac{d \sigma_{\circ}}{d \Omega^{*}}=\frac{\alpha^{2}}{s} \frac{\left(3+\cos ^{2} \theta_{C M}\right)^{2}}{\sin ^{4} \theta_{C M}},
$$

where $s=2 m e_{e}\left(E_{0}+m_{e}\right)$ is the Mandelstam variable, $\theta_{C M}$ is the CM scattering angle, and $E_{0}$ is the beam energy in the lab frame. Assuming that the beam direction is along the $\mathrm{Z}$-axis and that the scattering happens in the $\mathrm{ZX}$ plane, in the ultra-relativistic limit, $A_{i j}$ is:

$$
\begin{gathered}
A_{z z}=-\frac{\sin ^{2} \theta_{C M}\left(7+\cos ^{2} \theta_{C M}\right)}{\left(3+\cos ^{2} \theta_{C M}\right)^{2}}, \quad A_{x x}=\frac{\sin ^{4} \theta_{C M}}{\left(3+\cos ^{2} \theta_{C M}\right)^{2}}, \quad A_{y y}=-A_{x x}, \\
A_{z x}=A_{x z}=\frac{2 \sin ^{4} \theta_{C M} \cos \theta_{C M}}{\gamma\left(3+\cos ^{2} \theta_{C M}\right)^{2}}, \quad A_{x y}=A_{y x}=A_{z y}=A_{y z}=0
\end{gathered}
$$

where $\gamma=\sqrt{s} / 2 m_{e}$. For a brute-force Møller Polarimeter, where the target polarization is along the z-axis (beam axis) Equation 2.9 simplifies to

$$
\frac{d \sigma}{d \Omega^{*}}=\frac{d \sigma_{\circ}}{d \Omega^{*}}\left[1+P_{z}^{\mathrm{b}} A_{z z} P_{z}^{\mathrm{t}}\right]
$$

The cross-section asymmetry for beam and target spins aligned parallel and anti-parallel can be written now as

$$
A=\frac{\left(\frac{d \sigma}{d \Omega^{*}}\right)_{\uparrow \uparrow}-\left(\frac{d \sigma}{d \Omega^{*}}\right)_{\uparrow \downarrow}}{\left(\frac{d \sigma}{d \Omega^{*}}\right)_{\uparrow \uparrow}+\left(\frac{d \sigma}{d \Omega^{*}}\right)_{\uparrow \downarrow}}=P_{z}^{\mathrm{b}} P_{z}^{\mathrm{t}} A_{z z}\left(\theta_{C M}\right)
$$

In practice, a Møller polarimeter measures the rates, $N_{+/-}$, as the beam helicity is flipped. The detector acceptance is in a range of $\theta_{C M}$ and an average analyzing power is used, $\left\langle A_{z z}\right\rangle$. The acceptance is chosen to be centered about $\theta_{C M}=90^{\circ}$ where the magnitude of $A_{z z}$ reaches a maximum of $7 / 9$. To summarize, the relationship between the measured asymmetry, rates, analyzing power, beam polarization, and target polarization for a bruteforce Møller Polarimeter is

$$
A=\frac{N_{+}-N_{-}}{N_{+}+N_{-}}=P^{\text {beam }} \cdot P^{\text {foil }} \cdot\left\langle A_{z z}\right\rangle
$$

where $\left\langle A_{z z}\right\rangle$ is the mean analyzing power and I have abandoned the polarization subscripts with the understanding that they are both in the z-direction. Using equation 2.11 we 
can calculate the beam polarization if we know the foil polarization and the mean analyzing power by measuring the asymmetry.

\subsubsection{Key Elements}

From Equation 2.8 we can see that the magnitude of $\left\langle A_{z z}\right\rangle$ reaches a maximum of $7 / 9$ at $\theta_{C M}=90^{\circ}$ which is an important consideration when designing a Møller polarimeter. Choosing a design which has its acceptance centered around $\theta_{C M}=90^{\circ}$ allows the largest possible asymmetry to be measured. A unique challenge for this kinematic range is that the lab scattering angles are small, $\theta_{L a b}<3^{\circ}$ (see Figure 2.10), so a typical setup uses magnets to separate the scattered electrons from the undeflected beam. Systems at SLAC, MAMI, BATES and ELSA [47-53] use one magnet, either a quadrupole or dipole, which separate the Møller scatterers from the undeflected beam but also act as energy/momentum analyzers. The magnets function as a spectrometer by sorting the electrons according to their momentum via the Lorentz force, e.g. electrons with lower momentum bend more. The systems at JLab have more complex optics reach allow operation at a wide range of beam energies, Halls B and C uses multiple quadrupoles[14, 40, 41, 54, 55] while the newly upgraded Hall A system utilizes four quadrupoles and one dipole.

Before the scattered electron reach the detector the acceptance is generally defined at some collimation region. This is required because in order to precisely know the mean analyzing power the acceptance has to be well understood. The systems in Hall A and Hall $\mathrm{C}$ achieve this by using movable collimators, the system for Hall $\mathrm{C}$ is shown in Figure 2.11.

The detection system can also be classified into two basic types, single or double arm. In a single arm system only one of the scattered electrons are detected. These type of system were initially used at SLAC and BATES and achieved total uncertainties of $2.7 \%$ and $6.0 \%$ respectively. In the double arm system, both electrons are detected in coincidence which suppresses the background, mostly due to Mott scattering. Therefore for high precision polarimetry the double arm system is preferred.

The detectors must operate at a relatively high rate, therefore fast detectors such as plastic scintillators are typically used. Electromagnetic calorimeters are also used, allowing suppression of the low energy background [6]. If the detectors are segmented they can also be used to determine the energy of the scattered electron as a function of the position on the detector. The Hall $\mathrm{A}$ and $\mathrm{C}$ polarimeters are equipped with a calorimeters and plastic scintillator apertures, where the main asymmetry measurement is performed with the calorimeter. 


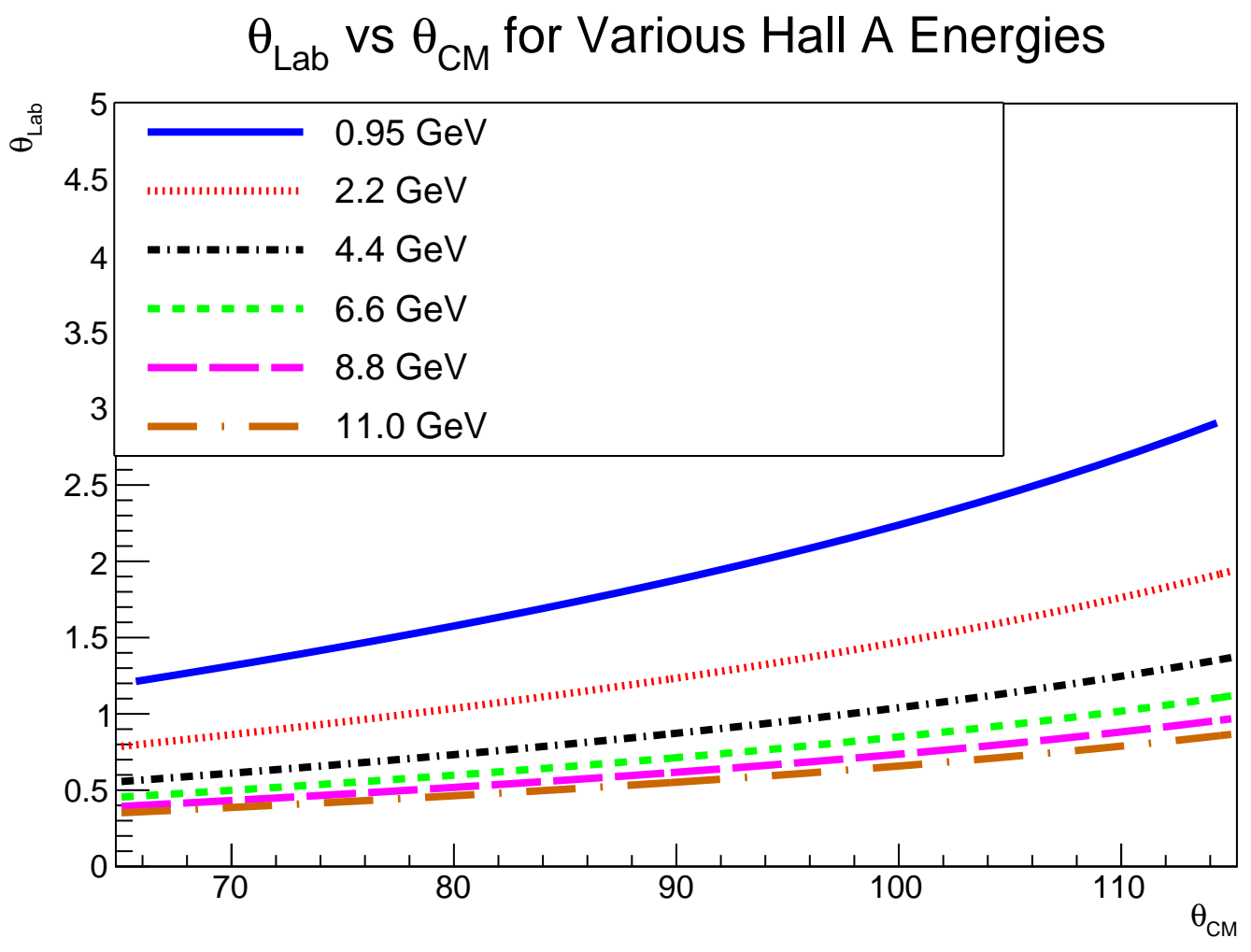

Figure 2.10: The Møller scattering angle in degrees of the lab frame vs the CM frame 

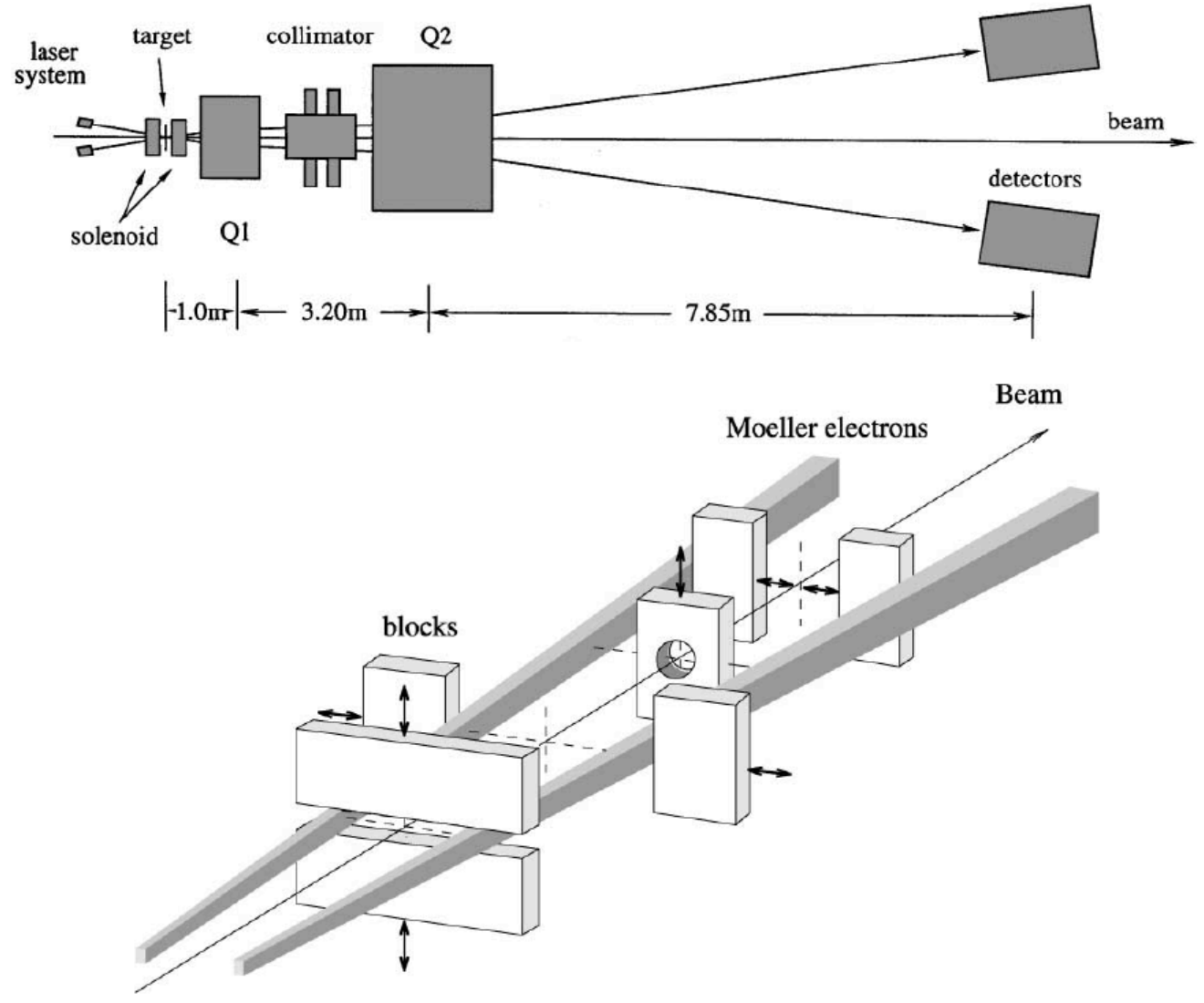

Figure 2.11: The Møller polarimeter in Hall C(top). Collimation region (bottom) 
The uncertainties associated with Møller polarimetry will be discussed in Chapter 4. The largest systematic include uncertainties in target polarization, analyzing power, and the Levchuk Effect [56]. The Levchuk effect is due to Møller scattering off of the (unpolarized) inner shell electrons in the target. These electrons have large binding energies, and therefore large momentum which can change the angle that the scattered electrons emerge from the target, which changes the acceptance of the detector. The result is that the effective target polarization is diluted, resulting in a larger measured asymmetry, and therefore a larger measured beam polarization. The Levchuk effect has been shown to cause a $14 \%$ effect to early polarimeters at SLAC [57] but since has been addressed in new polarimeter designs. It will be shown that the correction is at the $<1 \%$ level for the polarimeter in Hall A. Other systematic errors that will be addressed in Chapter 4 are dead time corrections, false asymmetries, target heating, and other uncertainties that can change the mean analyzing power like beam position, beam energy, and ill-defined geometries.

Table 2.1 is a list of Møller polarimeters at various accelerator facilitates. Much progress has been made over the past decades at controlling the systematic errors and constructing polarimeters that address the Levchuk effect. Technological advances in superconducting magnets allow for brute-force targets to be more practical which reduces the uncertainties of target polarization. With precise experimental modeling and well understood simulations the analyzing power can be accurately determined. In the next chapter I will discuss the polarimeter in Hall A and Monte Carlo simulation used.

The Hall A Møller polarimeter was first commissioned and used for experiment E93027, the results are shown in Figure 2.12. At the time $\delta p / p$ was $3 \%$. In 2015, the new target magnet and motion system was upgraded. The results for the DVCS experiment are shown in Figure 2.13 which shows the uncertainty, $\delta p / p$, drop from $2.2 \%$ with the tilted target to $1.1 \%$ with the "brute-force" target. 


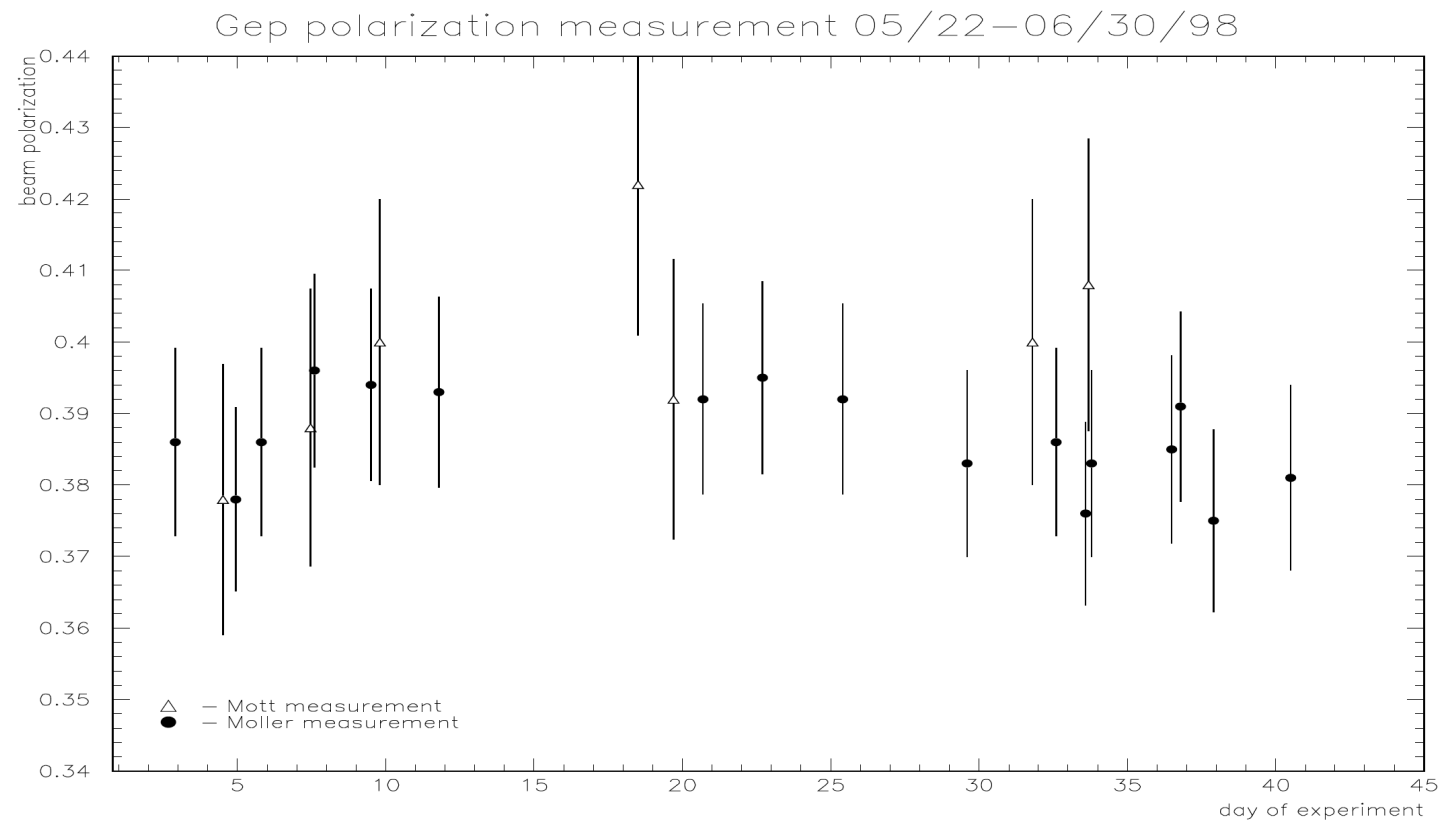

Figure 2.12: Result of the Hall A electron beam polarization measurements with Møller and Mott polarimeter for experiment E-93-027. This was the first reported results for the Møller polarimeter in Hall A. The Møller polarimeter initially reported the uncertainty (statistical and systematic) in a polarization measurement at $\leq 3 \%$ [10] 


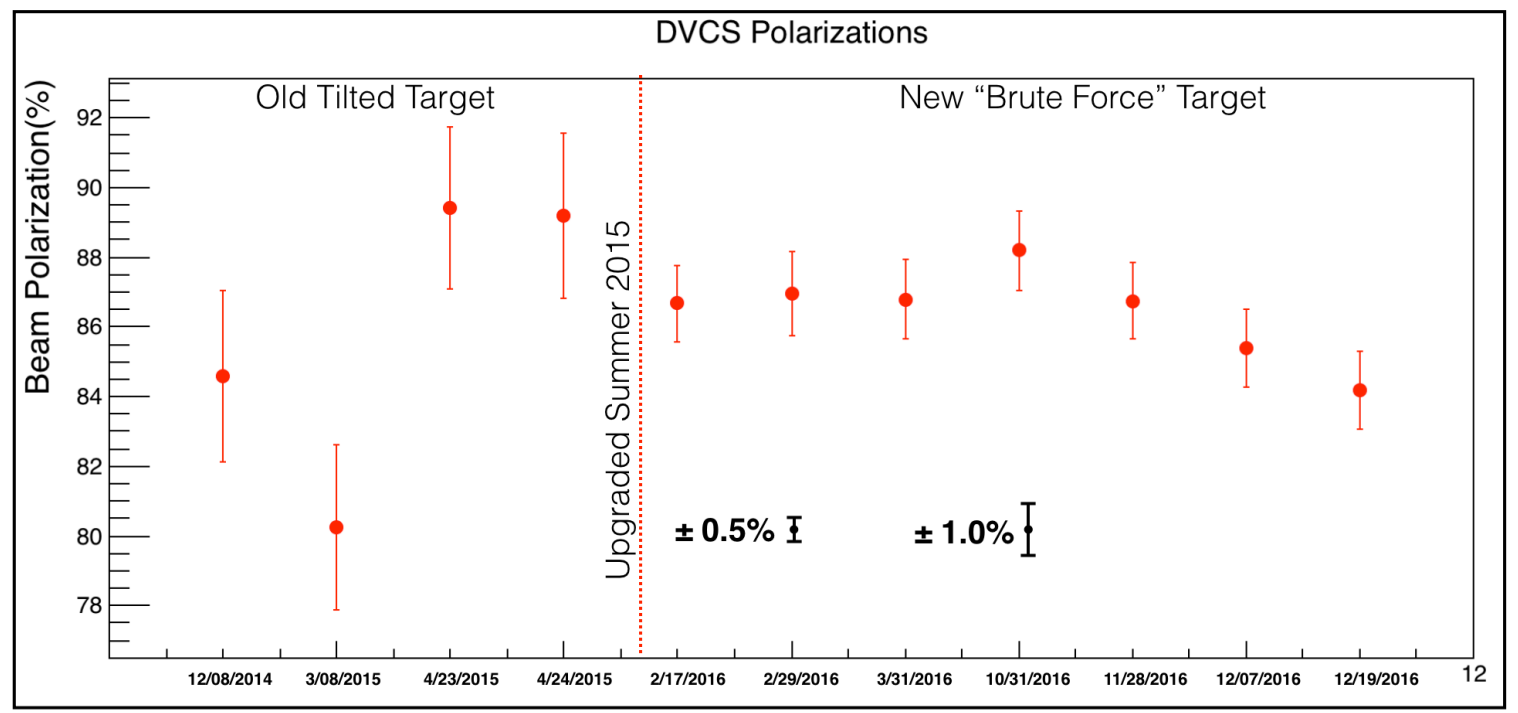

Figure 2.13: Polarization results before and after the upgrade. Measurements taken in Hall A of Jefferson Lab for The DVCS experiment 
Table 2.1: Møller Polarimeters at various laboratories and their associated errors

\begin{tabular}{|c|c|c|c|c|c|}
\hline \multirow{2}{*}{ Polarimeter } & \multirow{2}{*}{$\begin{array}{l}\text { Beam energy } \\
(\mathrm{GeV})\end{array}$} & \multirow{2}{*}{ Arms } & \multirow{2}{*}{ Optics } & \multicolumn{2}{|c|}{$(\delta P / P)_{\text {syst }}$} \\
\hline & & & & Target & Full \\
\hline SLAC[50] & 48 & 1 & $\mathrm{D}$ & $1.7 \%$ & $2.7 \%$ \\
\hline SLAC[51] & 16,29 & 2 & $\mathrm{D}$ & $2.3 \%$ & $2.4 \%$ \\
\hline MAMI[47] & 0.85 & 2 & Q & $2.0 \%$ & $9.0 \%$ \\
\hline MAMI[52] & 0. at $85-1.5$ & 2 & $\mathrm{D}$ & $0.6 \%$ & $1.6 \%$ \\
\hline Bates[48] & $0.25,0.57$ & 1 & Q & $1.25 \%$ & $6.0 \%$ \\
\hline Bates[49] & 0.87 & 2 & $\mathrm{Q}$ & $1.5 \%$ & $2.9 \%$ \\
\hline ELSA[53] & $1.0-3.3$ & 2 & $\mathrm{D}$ & $1.9 \%$ & $2.0 \%$ \\
\hline JLab, Hall A[9] & $0.85-6$ & 2 & QQD & $1.5 \%$ & $1.7 \%$ \\
\hline JLab, Hall A[9] & $0.85-6$ & 2 & QQD & $0.35 \%$ & $0.9 \%$ \\
\hline JLab, Hall B[54] & $0.85-6$ & 2 & QQ & $1.4 \%$ & $3.0 \%$ \\
\hline JLab, Hall C[41] (ideal) & $0.85-6$ & 2 & QQ & $0.3 \%$ & $0.5 \%$ \\
\hline JLab, Hall C[40] (Q-Weak) & $0.85-6$ & 2 & QQ & $0.3 \%$ & $0.8 \%$ \\
\hline JLab, Hall A & $0.85-11$ & 2 & QQQD & $0.3 \%$ & $1.0 \%$ \\
\hline
\end{tabular}




\section{CHAPTER 3}

\section{THE HALL A MØLLER \\ POLARIMETER APPARATUS AND MODELING}

This Hall A Møller polarimeter at Jefferson Laboratory was built by an international collaboration between University of Kentucky and the Kharkov Institute of Physics and Technology in Ukraine and was commissioned from 1997 to 1998. First measurements were performed for experiment E-93-027 which measured the electric form factor of the proton. At the time the beam polarization was measured to be $\sim 40 \%$ and a $\sim 3 \%$ uncertainty was reported. Over the last 30 years many upgrades have been made in order to reduce systematic errors and keep up with changing conditions in the hall. The latest upgrade was a new target motion system built by Temple University and new superconducting target holding field magnet purchased by Jefferson Lab. The motivation for the new system was to reduced the systematic error associated with target polarization. The upgrade was completed in 2015 and polarization measurements were made in 2015-2016 for The Deeply Virtual Compton Scattering (DVCS) experiment. Further improvements on the target system were made in 2016-2017 and installed in 2019. The polarimeter will be commissioned in March of 2019 and aims to provide 1\% polarimetry for PREX/CREX in the following months. The current apparatus will be described in the following section.

The key elements of the polarimeter (Figure 3.1), from target to detector are

- Superconducting split coil magnet and target motion system

- Four steering quadrupoles

- Lead collimators 
- Dipole

- Detector Assembly

\section{Hall A Møller Polarimeter}

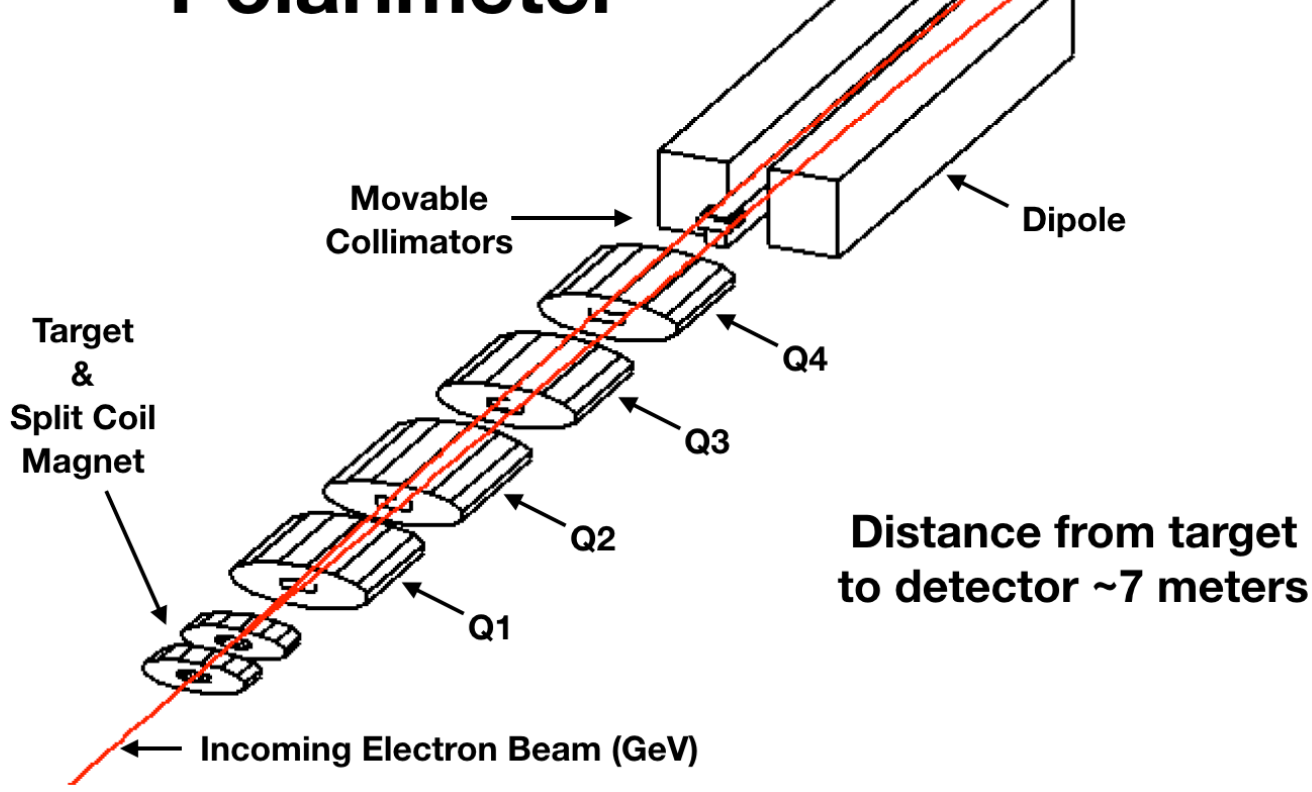

Figure 3.1: Hall A Møller Polarimeter at Jefferson Laboratory as modeled in Geant3. To give the reader an idea of the aspect ratio and scale, the quadrupoles have a circular cross-section with an outer diameter of $10 \mathrm{~cm}$ and the entire setup from target to detector is about $7 \mathrm{~m}$ long

The superconducting 5T split coil magnet is used to polarize the ferromagnetic target foils. The magnet provides a nearly uniform field in the same direction as the incident beam. The foils are oriented perpendicular to the field and are polarized out of plane so that the spins of the target electrons are aligned parallel or anti-parallel to the spins of the longitudinally polarized beam. The motion system has two degrees of freedom, it enables users to insert one of four foils in the beamline while also providing rotational control of the target ladder. The rotation is used to ensure that the foils are normal to the beamline and for performing systematic tests. The angular precision is $<0.1^{\circ}$. 
The polarimeter is designed to accept Møller scattered electrons centered about the center of mass angle $\theta_{C M}=90^{\circ}$ or equivalently electrons with energies equal to half that of the incident electron beam. Both final state electrons are detected in coincidence thereby reducing backgrounds primarily from Mott scattering off the target nucleus. The Møller scattered electrons leave the target area and are steered by four quadrupole magnets. Typical Møller electrons of interest are scattered at an angle of less than $3^{\circ}$ from the incident beam so the magnets are required in order to steer the particles through the remainder of the system. The optical configuration for the polarimeter is unique for each beam energy and needs to be optimized from simulation in advance. The term "optical configuration" refers to the set current in each of the four quadrupole magnets as well as the dipole magnet. It is also worth noting that the configuration changes slightly depending upon the magnitude of the target holding field.

The limiting aperture for the scattered events in the horizontal direction turns out to be the beam pipe upstream of the dipole and the thin apertures through the dipole. A precision vertical lead collimator just upstream of the dipole limits the acceptance in the vertical direction.

By the time the electrons have entered the dipole a majority of the final acceptance has been determined. The dipole field is in the horizontal direction so that the electrons are bent downwards by the Lorentz force. The electrons are momentum analyzed by the dipole and then exit via two thin titanium windows.

At this point the electrons have left vacuum and drift through open air until they reach the detector box assembly. The outer layer of the assembly is a protective shielding box made of thick lead walls with an entrance for the electrons to pass in the upstream face. The detector package comprises two identical symmetrically placed detectors. Each side or arm of the detector consists of two spaghetti-lead calorimeter blocks with two photomultiplier tubes (PMT's) on each block for a total of 8 PMT's. In 2010, plastic scintillator paddles were added in front of the left and right arm detectors. Coincidence events can be determined with and without using the paddles.

The entire polarimeter has been modeled with Monte Carlo simulations. The simulation is required to determine the magnet settings and the acceptance of the detector. Knowledge of the acceptance is required to determine the analyzing power which is then used in the final polarization calculation. The Monte Carlo used is COMGEANT, which is an interface to the Geant3 simulation package developed at CERN. It allows users to add custom geometry files that are read at run time which eliminates the need for recompiling the code each 
time a change is made. The package is written with FORTAN and analysis is done with PAW style kumac files. COMGEANT has been used for the Møller polarimeter in Hall A since 1998 and is currently being phased out in favor of a GEANT4 simulation which utilizes the more modern $\mathrm{C}++$ computer language and the ROOT analysis framework.

In the remainder of this chapter, each component will be described in detail and its implementation in Geant3/4.

\subsection{Superconducting Split Coil Magnet}

The iron target is placed in a super conducting split coil magnet with the magnetic field perpendicular to the foil's surface and parallel to the beam line. The configuration of the split coil magnet is approximately a Helmholtz configuration where both coils are a circle and the spacing between then is equal to their radius. In this configuration the second derivative of $B_{z}(z)$ equals 0 , therefore $B_{z}$ is especially flat in the center where the target is found. The purpose of the magnet is to magnetize/polarize the foil (out-of-plane) thereby aligning the spins of the polarizable electrons parallel or anti-parallel to the incident beam electron's spin. The Geant 3 simulation can implement the field by either using a field map or a uniform field along the beam line direction. Generally a field map is used and the uniform field option is only implemented for testing and debugging the code. The field map is preferred because it includes the small the radial component of the field and more accurately describes the longitudinal field. This is important because both the radial field and the longitudinal field have an impact on the final acceptance of the detector.

The split coil magnet was mapped at JLab before it was installed in the beam line. The purpose of the mapping was to determine if the mechanical and magnetic axes of the magnet were the same. The longitudinal magnetic field was measured along the bore of the magnet from $\mathrm{z}=-26 \mathrm{~cm}$ to $\mathrm{z}=26 \mathrm{~cm}$ in $0.5 \mathrm{~cm}$ steps. At each $\mathrm{z}$ location the field was measured at 7 points along the $x$ (horizontal) and $y$ (vertical) axes. The magnetic center $(\mathrm{x}, \mathrm{y})$ was then calculated at each $\mathrm{z}$ location by plotting $B_{z}$ vs $x$ or $y$, fitting the points to a second order polynomial, and finding the location of the maximum or minimum (See Figure 3.2 to see how the $\mathrm{z}$ location determines if a maximum or minimum is found). If the magnetic axis was perfectly aligned with the mechanical axis the magnet the points would fall on a straight line at $x=0$ and $y=0$. The data here can be explained by adding a tilt and offset to each coil which is shown by the red squares in figure 3.4. The data along the $\mathrm{z}$-axis was fit to the theoretical equations for a split coil magnet from [58]. These equations 

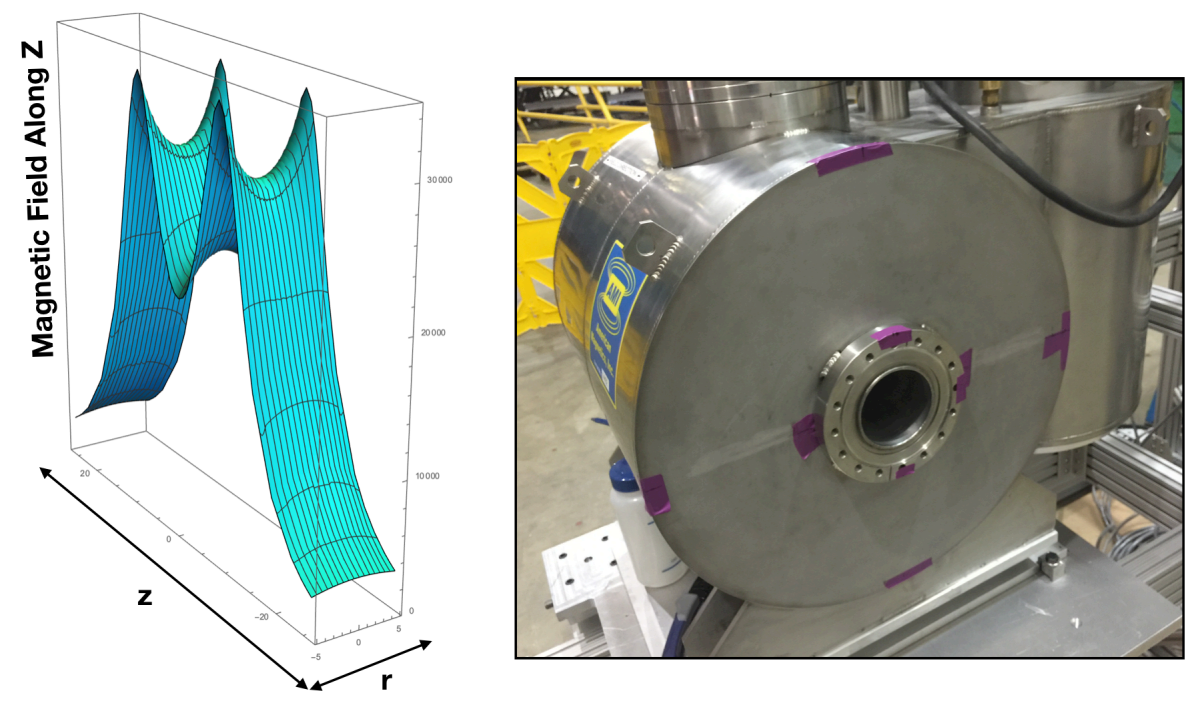

Figure 3.2: (Left) The magnetic field strength inside the bore of the split coil magnet(right). To determine the magnetic axis of the magnet the transverse minimum or maximum at different $\mathrm{z}$ locations were determined from magnetic field mapping measurements.

required fitting the data to determine the three parameters which were the current in the coils, the radius of the coils and the spacing between the coils. The fit and data is shown in figure 3.3. Once the three parameters of the fit were determined, it could be used to create field maps that included the radial components of the field. The fit is plotted in figure 3.4 where the data is represented by the green triangles. The conclusions of this study were that the mechanical and magnetic axes were aligned at the $100 \mu \mathrm{m}$ and $0.1^{\circ}$ level.

The target field also produces some undesirable effects in the polarimeter due to the Lorentz force acting on the scattered electrons which twists the scattered electrons about the beam axis. Note that the contribution to this effect is both from $\vec{v}_{r} \times \vec{B}_{z}$ and $\vec{v}_{z} \times \vec{B}_{r}$ which twist in the same direction in the central region of the magnet after the target and in opposite directions in the outer regions where the $B_{r}$ component changes signs. This twisting effect can be seen on the detector plane in Figure 3.5 after the electrons are bent downward by the dipole magnet. Here the electrons leave the target area with an additional counter-clockwise twist about the beam axis. Looking down the beam line from the target area, the electrons on the left have an additional mostly downward momentum which stretches the area on the detector which the electrons hit. On the right the twist gives and additional momentum in mostly the upward direction which compresses the area on the detector where the electrons 


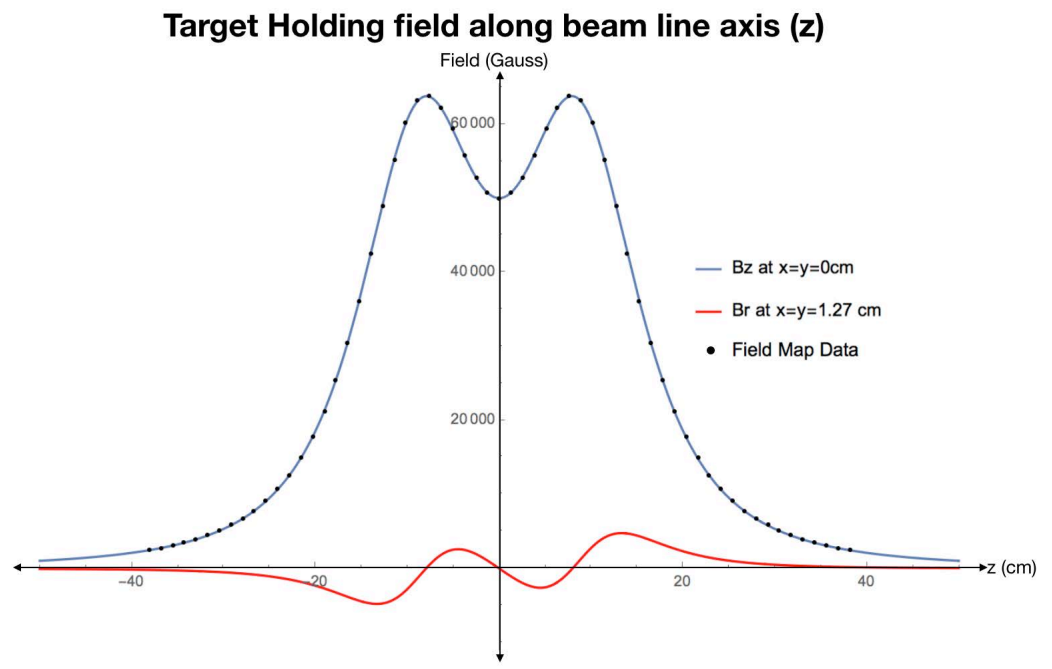

Figure 3.3: Measured data and fit of holding field magnet. Once the fit was determined the radial component could be computed. The red line shows the radial component at $1.27 \mathrm{~cm}$ off from the $\mathrm{z}$-axis. The target foils are located at $\mathrm{z}=0 \mathrm{~cm}$.

Field Mapping Data and Calculated Results for Misaligned Coils

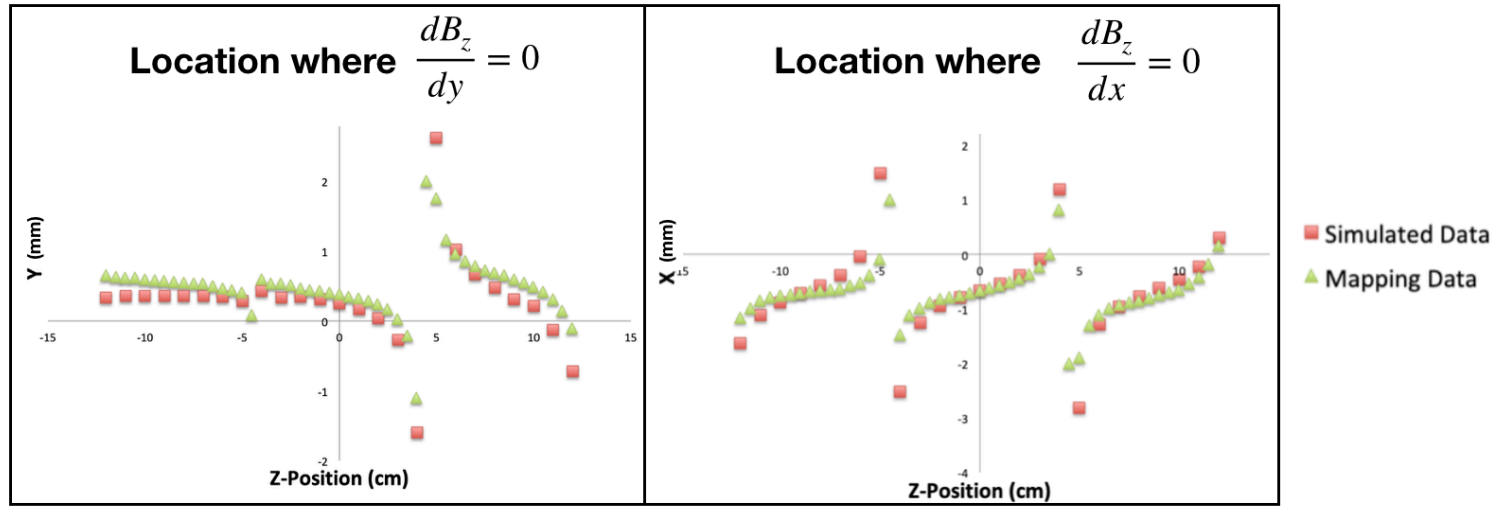

Figure 3.4: By including offsets and rotations to each of the coils, the magnet mapping data could be explained. The figure on the left had one coil shifted $0.4 \mathrm{~mm}$ and rotated $0.25^{\circ}$ and the figure on the right has both coils shifted $0.67 \mathrm{~mm}$ and rotated $0.23^{\circ}$. Ideal coils that were perfectly aligned would have a line at $x=0$ and $y=0$. The discontinuities are at the points where the saddle point changes from negative to positive curvature and have essentially 0 slope everywhere so there is no sensitivity to find a precise location in $\mathrm{x}$ and $\mathrm{y}$. 
hit.

Singles

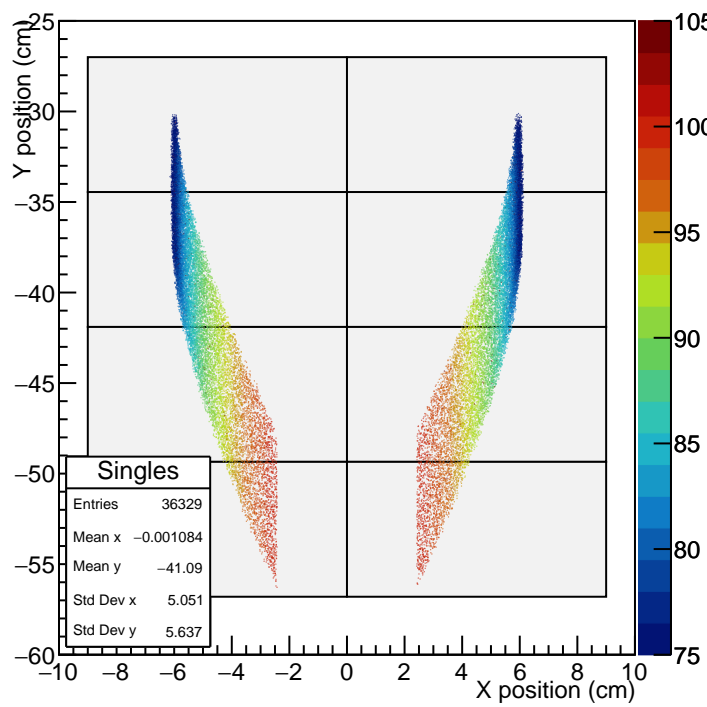

Coincidence $\mathrm{x} / \mathrm{y}$

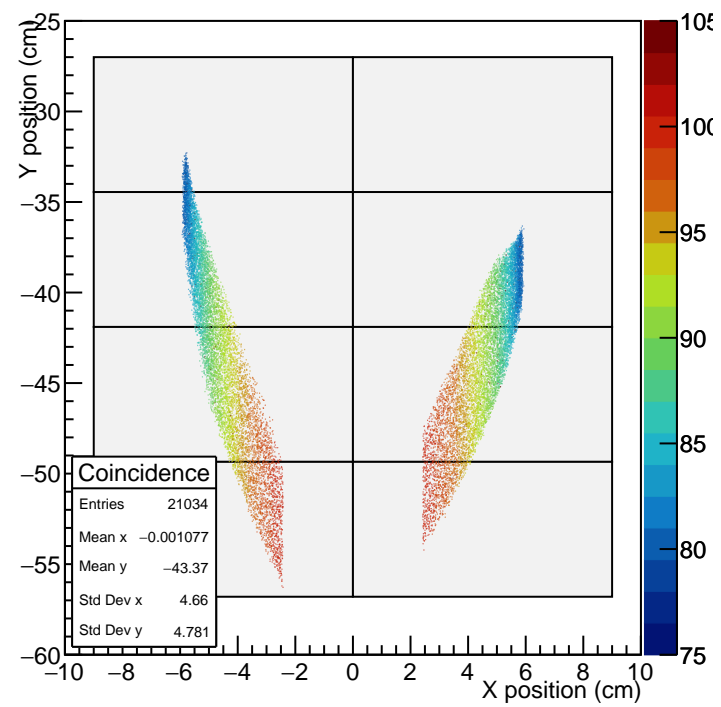

Figure 3.5: Coincidence and single electrons on detector at $0.95 \mathrm{GeV}$

\subsection{Target Motion System}

A new target motion system was required for the new split coil magnet. Two systems were built at Temple University. The first system was installed in 2015 and took polarization measurements through 2016 for the DVCS experiment. During a period of unpolarized experiments in Hall A the magnet was removed from the hall and a more robust target motion system was developed and built at Temple University. The new system is currently installed in the Hall and will be used for future polarization measurements.

One of the challenges in designing a beam line motion system is creating motion in the vacuum of the beam line while having the motors outside the beam line. The linear motion is achieved by using a bellow which expands and contracts as the targets are inserted and removed from the beam. Rotation of the target ladder was made possible by a rotational feed thru which uses a system of replaceable o-rings to maintain vacuum. Due to the high torques associated with inserting an iron target into a 4T field the new motion system (Figures 3.6 and 3.9) features some additional improvements. A power-off brake was implemented to lock the rotation when not in use, thereby removing any unnecessary strain 
to the motor. The stepping motor also features a 50:1 gear box which further decreases the load on the motor while increasing its lifetime.

The position and angle of the ladder is determined by a pair of string potentiometers. As the target ladder is translated or rotated the end of the string is moved changing the resistance of the potentiometer. This corresponds to a voltage change across the wiper of the potentiometer which is then converted into an angle or position. The linear resolution of the system is $<0.5 \mathrm{~mm}$ and the angular resolution is $\sim 0.1^{\circ}$.

The target ladder is shown in figure 3.7 with 1,4 , and $10 \mu \mathrm{m} \mathrm{Fe} \mathrm{foils} \mathrm{installed.} \mathrm{The}$ foil holders are removable to allow for foils of varying thicknesses and material (nickel or supermendur) to be used. Two tapped holes in the main shaft where the ladder attaches allow for removal and replacement of the ladder while not losing its absolute position within the beamline.

The iron foils are purchased commercially, e.g. Goodfellow Corp, with a purity of $99.85 \%$ to $99.99 \%$. The fractional uncertainty of the thickness is $\sim 10 \%$. The foils may be annealed, for instance the $12.5 \mu \mathrm{m}$ Fe foil used for the DVCS polarization measurements was annealed for 1 hour in vacuum at $930^{\circ} \mathrm{C}$. The foils are rolled to the desired thickness and therefore have randomly oriented crystal domains. It is assumed that a single magnetic domain is formed when the foils are saturated out-of-plane using a 3-4 T magnetic field. The rational behind this can be explained by a quote from An Introduction to Magnetic Materials by Cullity and Graham[59].

Crystal anisotropy is due mainly to spin-orbit coupling. By coupling is meant a kind of interaction. Thus we can speak of the exchange interaction between two neighboring spins as a spin-spin coupling. This coupling can be very strong, and acts to keep neighboring spins parallel or antiparallel to one another. But the associated exchange energy is isotropic; it depends only on the angle between adjacent spins, as stated by Equation 4.29, and not at all on the direction of the spin axis relative to the crystal lattice. The spin-spin coupling therefore cannot contribute to the crystal anisotropy.

The orbit-lattice coupling is also strong. This follows from the fact that orbital magnetic moments are almost entirely quenched, as discussed in Section 3.7. This means, in effect, that the orientations of the orbits are fixed very strongly to the lattice, because even large fields cannot change them.

There is also a coupling between the spin and the orbital motion of each electron. When an external field tries to reorient the spin of an electron, the orbit 
of that electron also tends to be reoriented. But the orbit is strongly coupled to the lattice and therefore resists the attempt to rotate the spin axis. The energy required to rotate the spin system of a domain away from the easy direction, which we call the anisotropy energy, is just the energy required to overcome the spin-orbit coupling. This coupling is relatively weak, because fields of a few hundred oersteds or a few tens of kilamps per meter are usually strong enough to rotate the spins. Inasmuch as the lattice consists of a number of atomic nuclei arranged in space, each with its surrounding cloud of orbital electrons, we can also speak of a spin-lattice coupling and conclude that it too is weak.

Relative magnetization measurements have been performed on the target foils using a Kerr effect apparatus. The setup measured the amount the polarization Future measurements are being considered such as measuring the magnetization of single crystal Fe disks relative to a polycystalline foil to verify that saturation that the magnetization is independent of crystal structure.
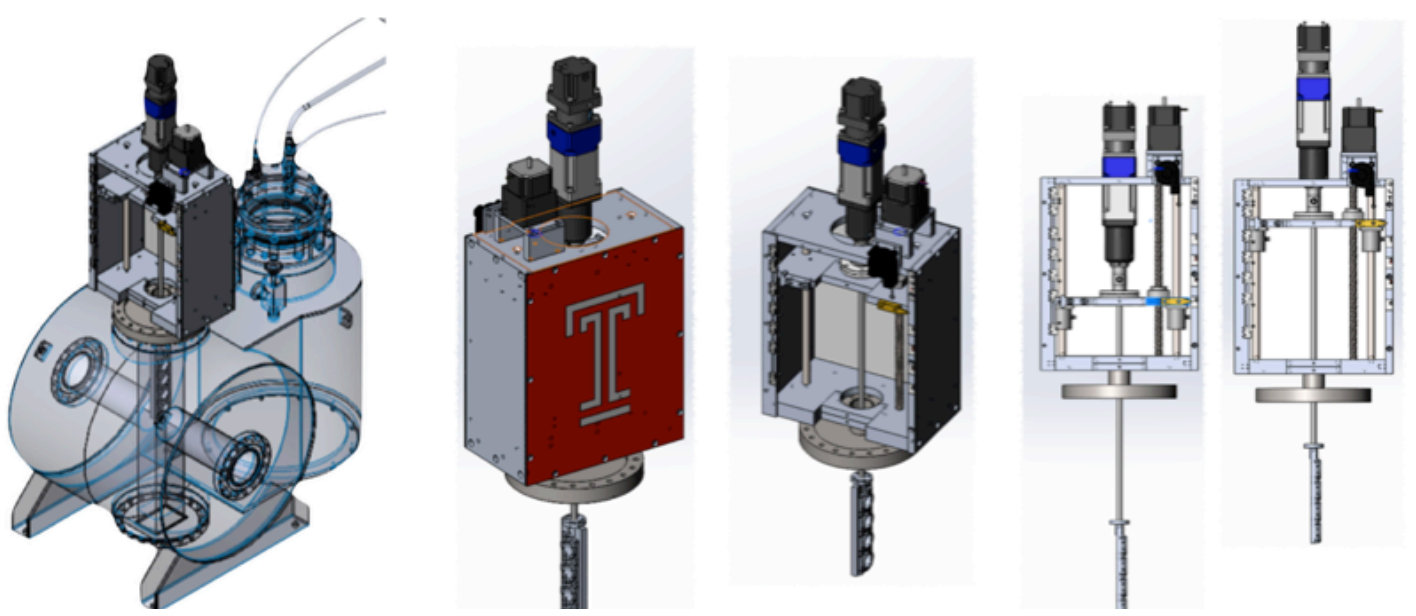

Figure 3.6: Temple University Møller Target Motion System in the design phase (2016). Engineered and drawn by James Wilhemi 


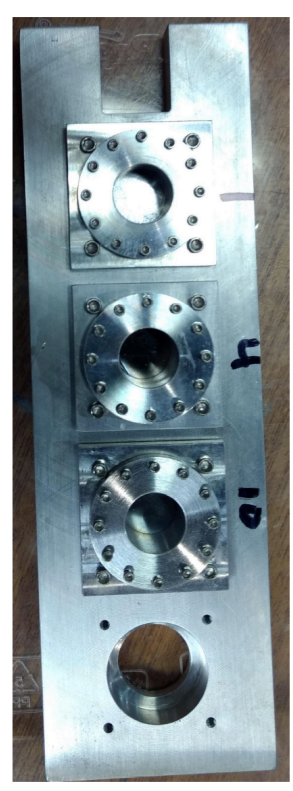

Figure 3.7: Møller target ladder with 1, 4, and $10 \mu \mathrm{m}$ foils (2018)

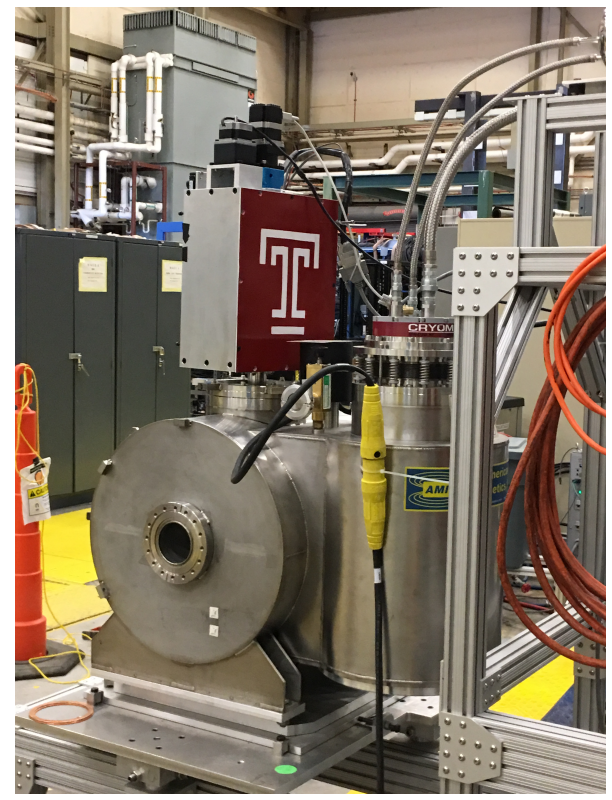

Figure 3.8: New Møller target system installed on the superconducting split coil magnet(2017)

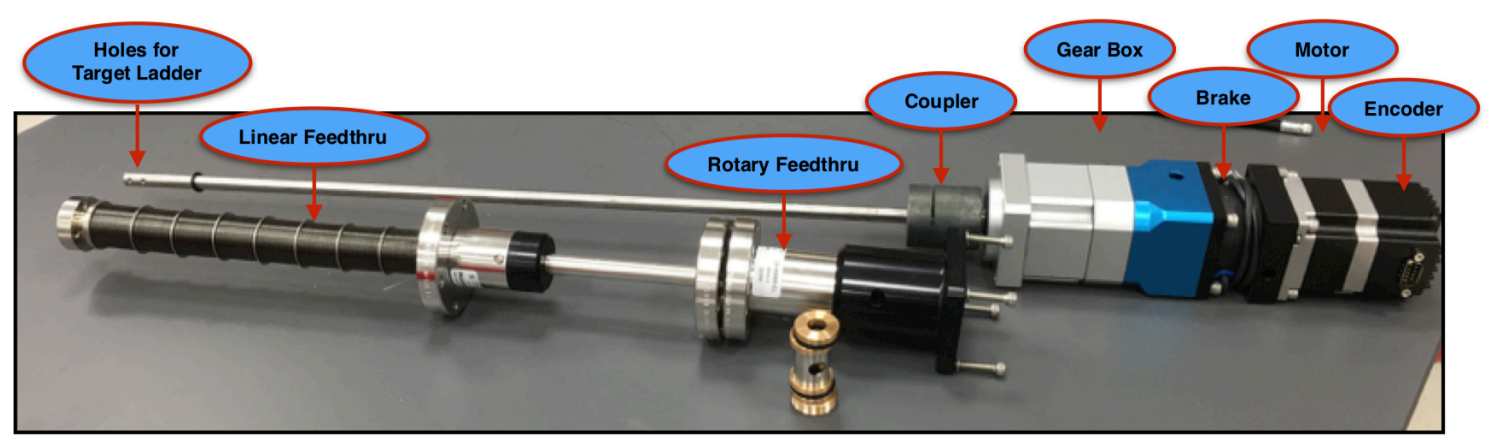

Figure 3.9: Main components of target motion system which allow rotation and translation of the target foils. Two feed-thru's are required since the target is under vacuum and the motors are located outside vacuum. An additional rotational encoder was added and not shown here. 


\subsection{Quadrupoles}

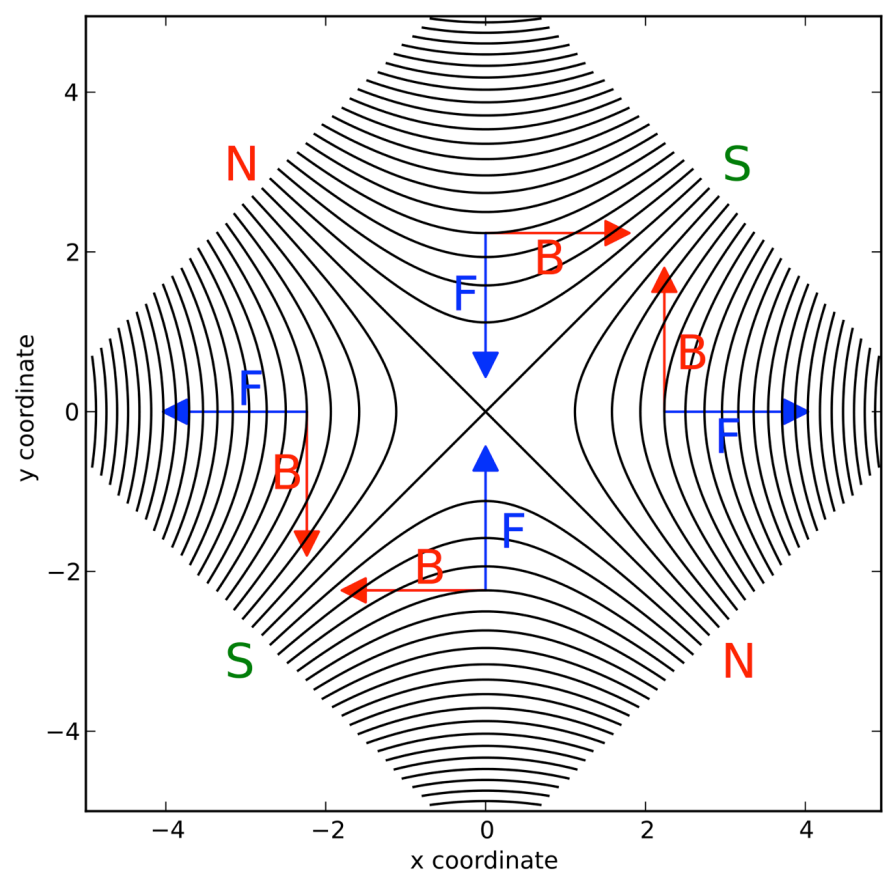

Figure 3.10: The magnet field of an ideal quadrupole and force it exerts on a moving charged particle[11]

Four quadrupole magnets are used to steer the Møller scattered electrons from the target into the dipole box. Depending on the direction of the current through a quadrupole, they can either be focusing in the horizontal direction (defocusing in the vertical direction) or focusing in the vertical direction (defocusing in the horizontal direction). Figure 3.10 shows an ideal quadrupole field and the force exerted on a positive charged particle traveling into the page. At low beam energies the quadrupoles are set to focus in the horizontal direction. At higher beam energies, where $\theta_{C M}=90^{\circ}$ becomes small, at least one of the quadrupoles must be defocusing in the horizontal direction.

Initially three quadrupoles from Los Alamos National Lab were used and a custom 
made fourth quadrupole was added for the $11 \mathrm{GeV}$ era upgrade. The new quadrupole, Q1, is furthest upstream followed by Q2, Q3 and Q4. The original quads now called Q2, Q3, and Q4, were mapped years ago at Los Alamos Laboratory. The new magnet, Q1, was mapped at JLab along with additional limited measurements of Q2. With this information the effective lengths of the magnets were determined along with the fields' current dependence. The current settings for these magnets must be optimized for each beam energy. Prior studies for the $11 \mathrm{GeV}$ era have produced the results shown in 3.11. These settings were calculated for the $11 \mathrm{GeV}$ upgrade at JLab and although they work well at higher energies figure 3.12 shows they are not optimized for lower beam energies (see setting at $1.1 \mathrm{GeV})$.

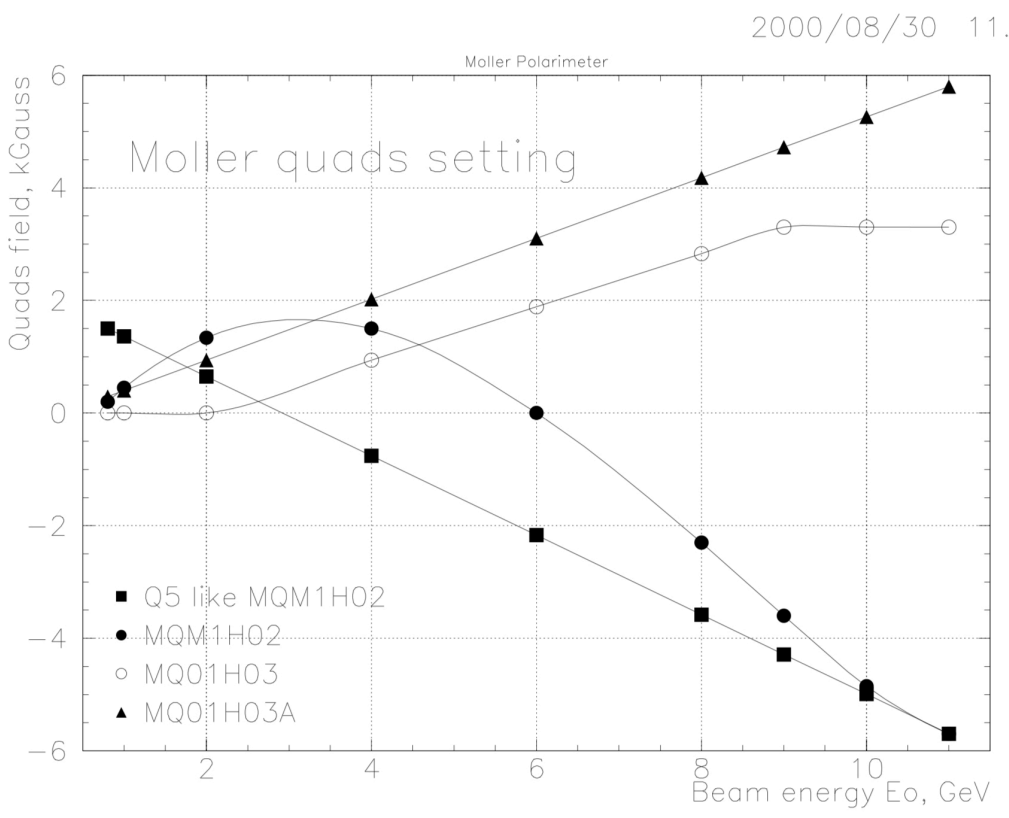

Figure 3.11: Suggested quadrapole settings as a function of electron beam energy https: //hallaweb.jlab.org/equipment/moller/docs/upgrade_llgev.pdf

In the Geant 3 simulation the quadrupoles are implemented as ideal quadrupoles. The field for an ideal quadrupole is

$$
\begin{aligned}
& B_{y}=K x \\
& B_{x}=K y
\end{aligned}
$$

where $K$ is the field gradient and considered to be a constant. The effective length and field 


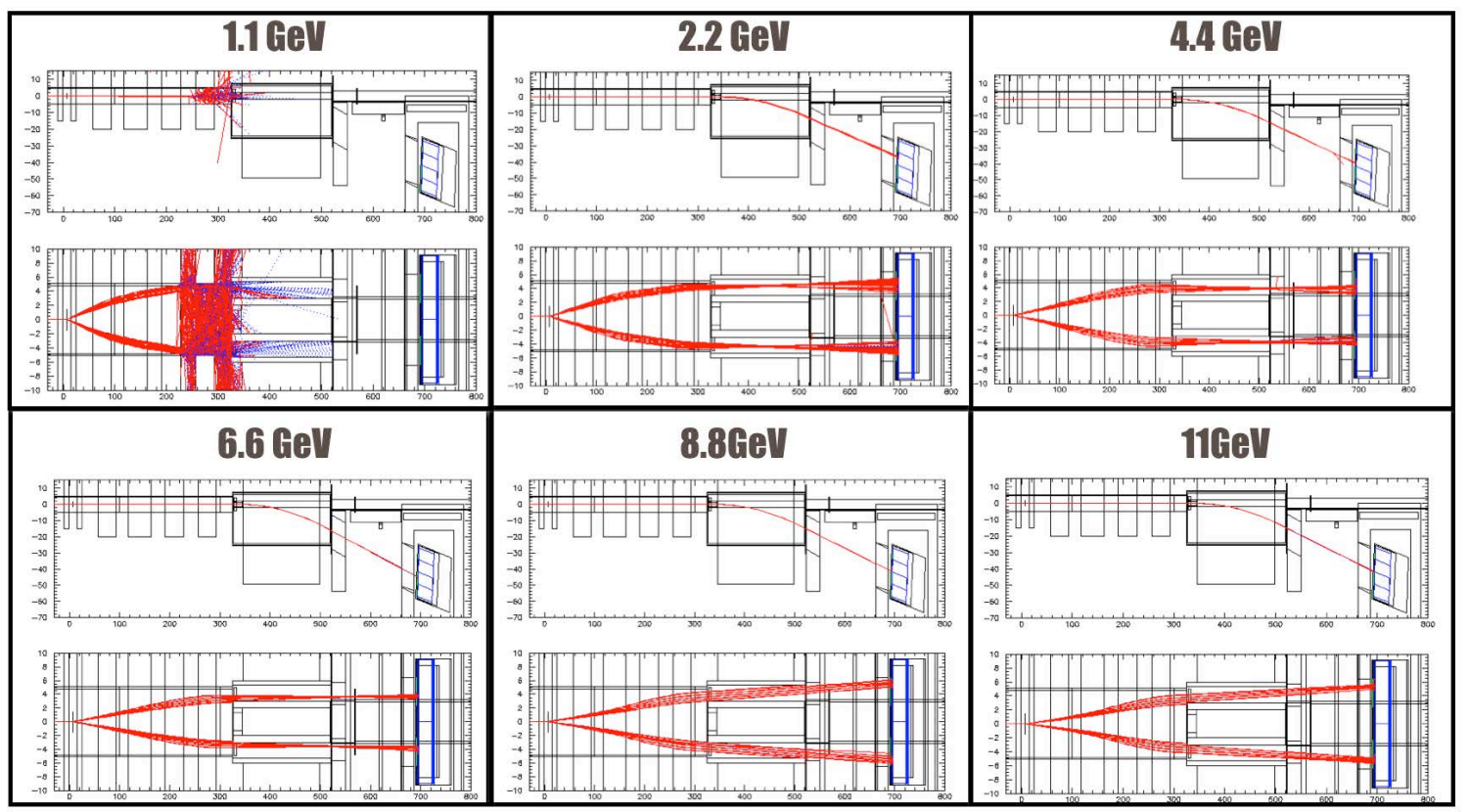

Figure 3.12: Results when using the solution in Figure 3.11 to determine quadrupole settings at various nominal beam energy settings

at the pole tip of the magnet are the only parameters required by the simulation. Figure 3.13 shows mapping data taken with a Hall probe for the new Q1 magnet. The effective length was calculated by integrating the field along the length of the magnet and dividing it by the field at the center $\left(Z_{e f f}=\int B d l / B_{\text {center }}\right)$. Similar measurements were performed for the other quadrupoles. 


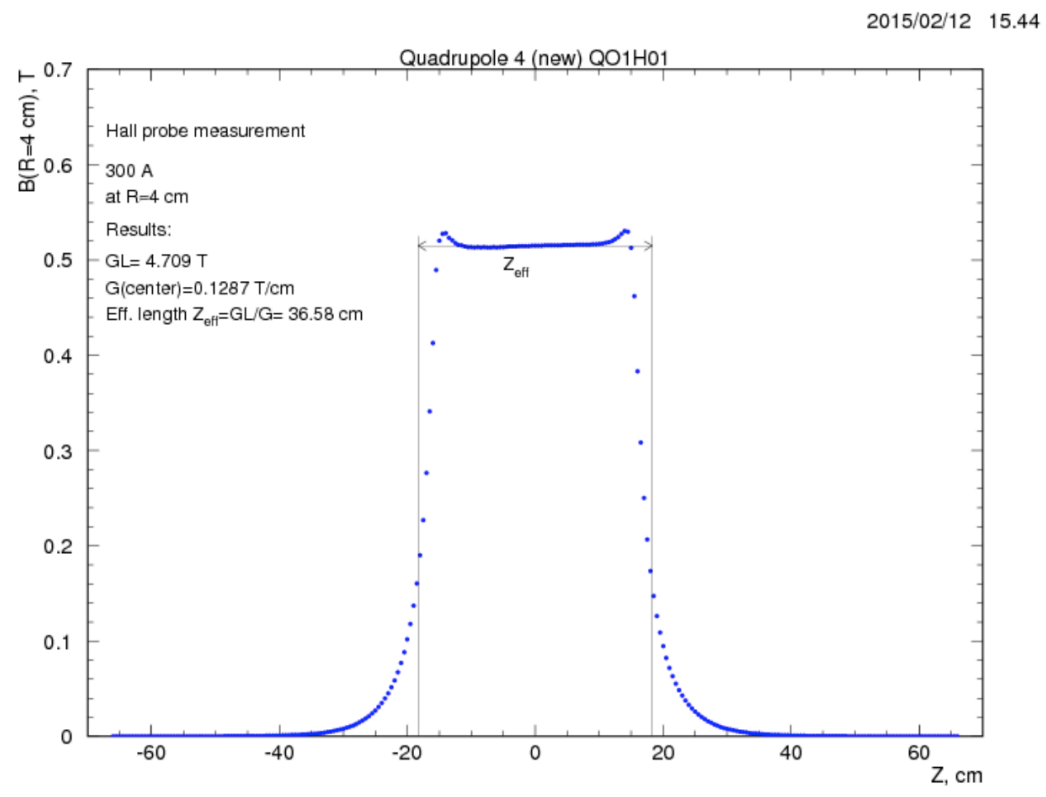

Figure 3.13: Determination of the effective length used in the simulation from mapping measurements [12]

\subsection{Dipole Box}

The next region in the spectrometer is the dipole. The Møller electrons enter the dipole region through two openings $\sim 2.3 \mathrm{~cm}$ wide. Two vertically adjustable collimators determine the height of the openings which can be set from $0.0 \mathrm{~cm}$ to $4.0 \mathrm{~cm}$ The settings of the quadrupoles kinematically select which electrons will pass through the two openings. The entrance to the dipole vacuum box (with beam pipe removed) is shown in Figure 3.14 where you can see the ConFlat flange, movable collimators and magnetically shielded beam pipe for the undeflected beam. The dipole vacuum box is $11 \mathrm{~cm}$ wide and contains an additional steel box $6 \mathrm{~cm}$ wide and centered in the vacuum box. There is a $2.295 \mathrm{~cm}$ wide gap on either side of the center box where the electrons travel through the magnetic field which is in the horizontal direction. The electrons are bent downwards and leave the dipole box through two $16 \mathrm{~cm} \times 2.36 \mathrm{~cm} 100 \mu \mathrm{m}$ thick titanium windows. Immediately after the dipole exit there is lead shielding (Figure 3.15) containing two slits for the electrons to travel through. 

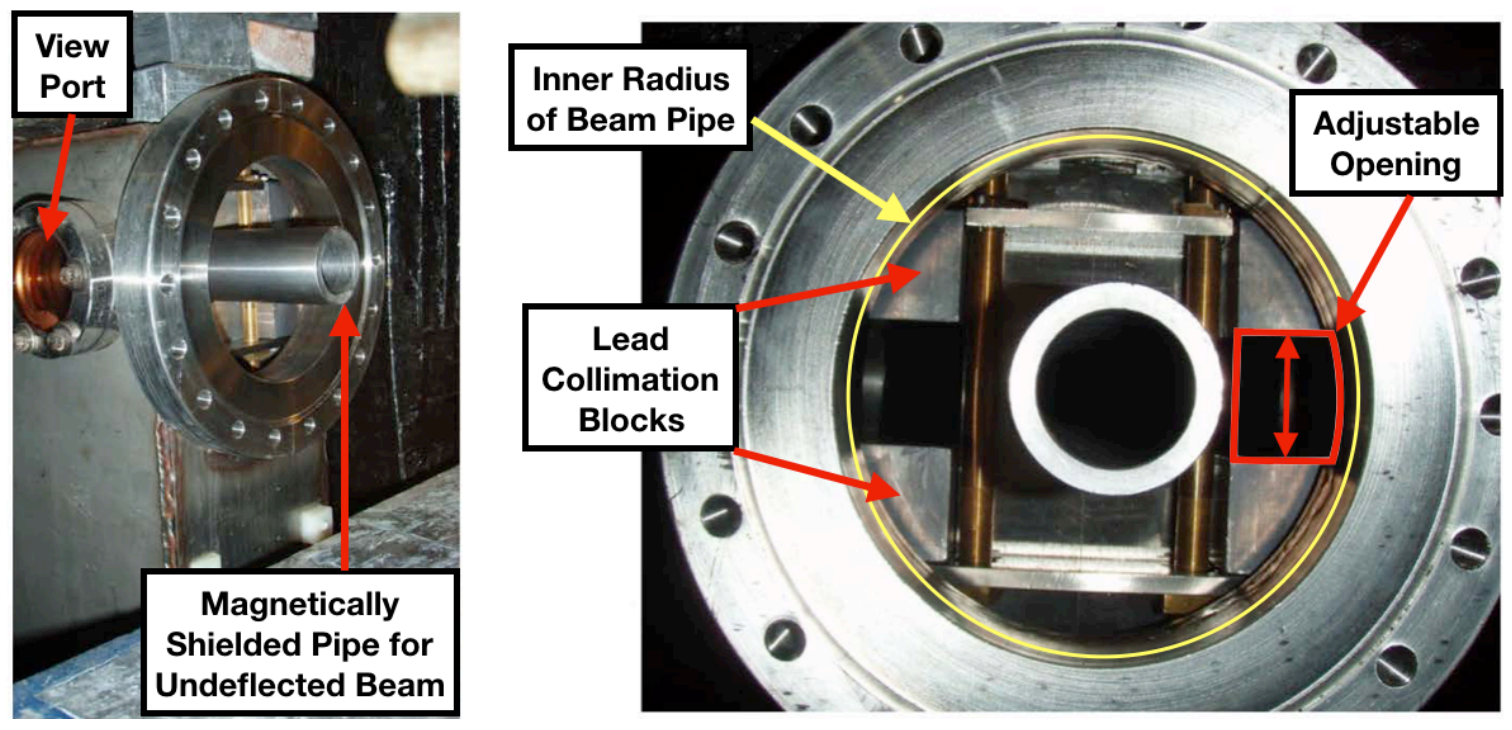

Figure 3.14: Dipole entrance and collimators 

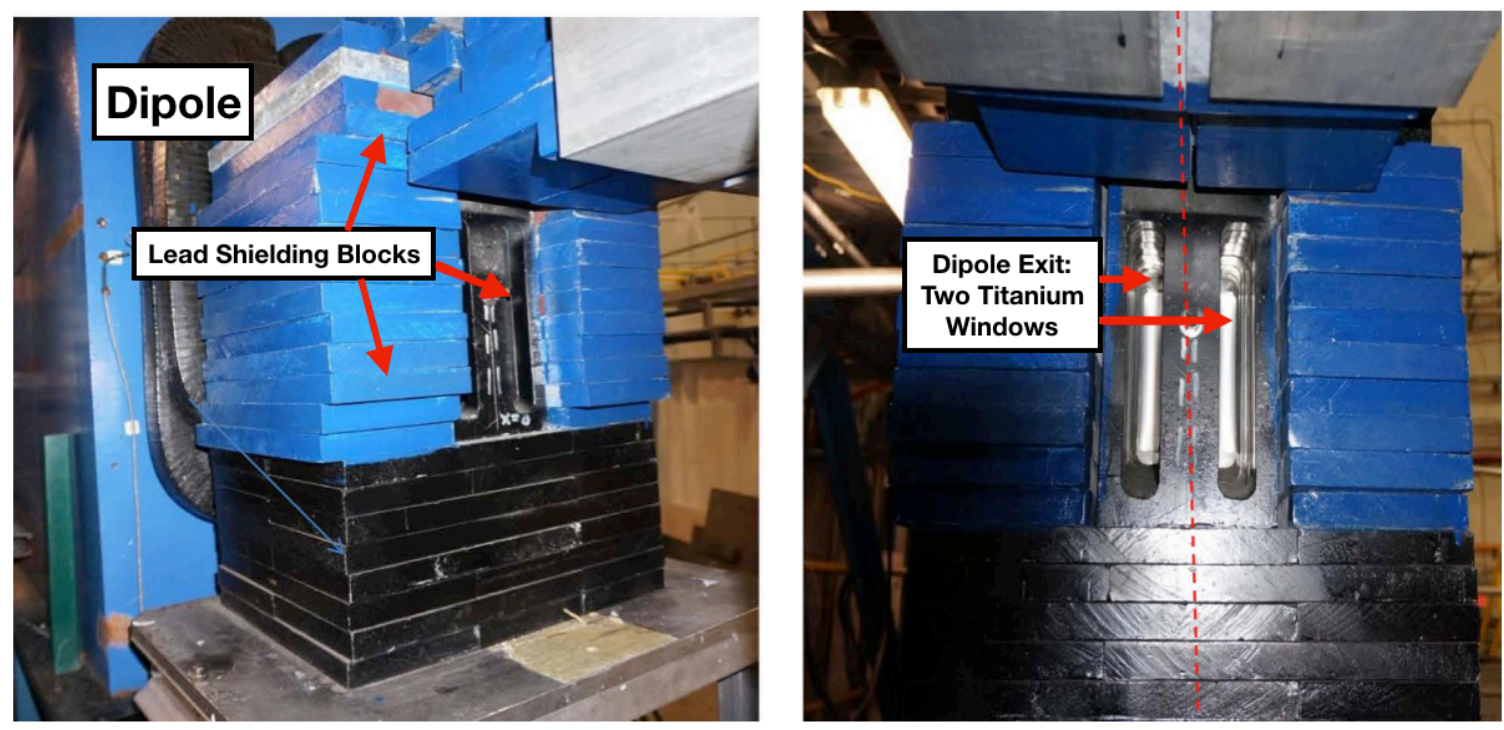

Figure 3.15: Lead shielding at dipole exit 


\subsection{Detector Box}

The main detectors are located inside a box made of lead shielding. The shielding box is modeled in the simulation as a $74 \mathrm{~cm} \times 94.2 \mathrm{~cm} \times 122.4 \mathrm{~cm}$ lead box with a wall thickness of $22 \mathrm{~cm}$. On the upstream face of the box there is a $13 \mathrm{~cm} \mathrm{x} 28 \mathrm{~cm}$ entrance window for the electrons to travel to the detector. The calorimeter is located inside the lead box and is modeled as eight blocks with each face measuring $9 \mathrm{~cm} \times 7.5 \mathrm{~cm}$ and $30 \mathrm{~cm}$ long. In front of the calorimeter, on beam right and beam left, are two scintillator paddles each $4 \mathrm{~cm}$ wide and $31 \mathrm{~cm}$ high. Geant 3 simulates the signal (energy deposited) in the calorimeter. Hits on the detector may be determined in the simulation by looking at the end of the tracks, the signal deposited in the calorimeter, or hits in the calorimeter.

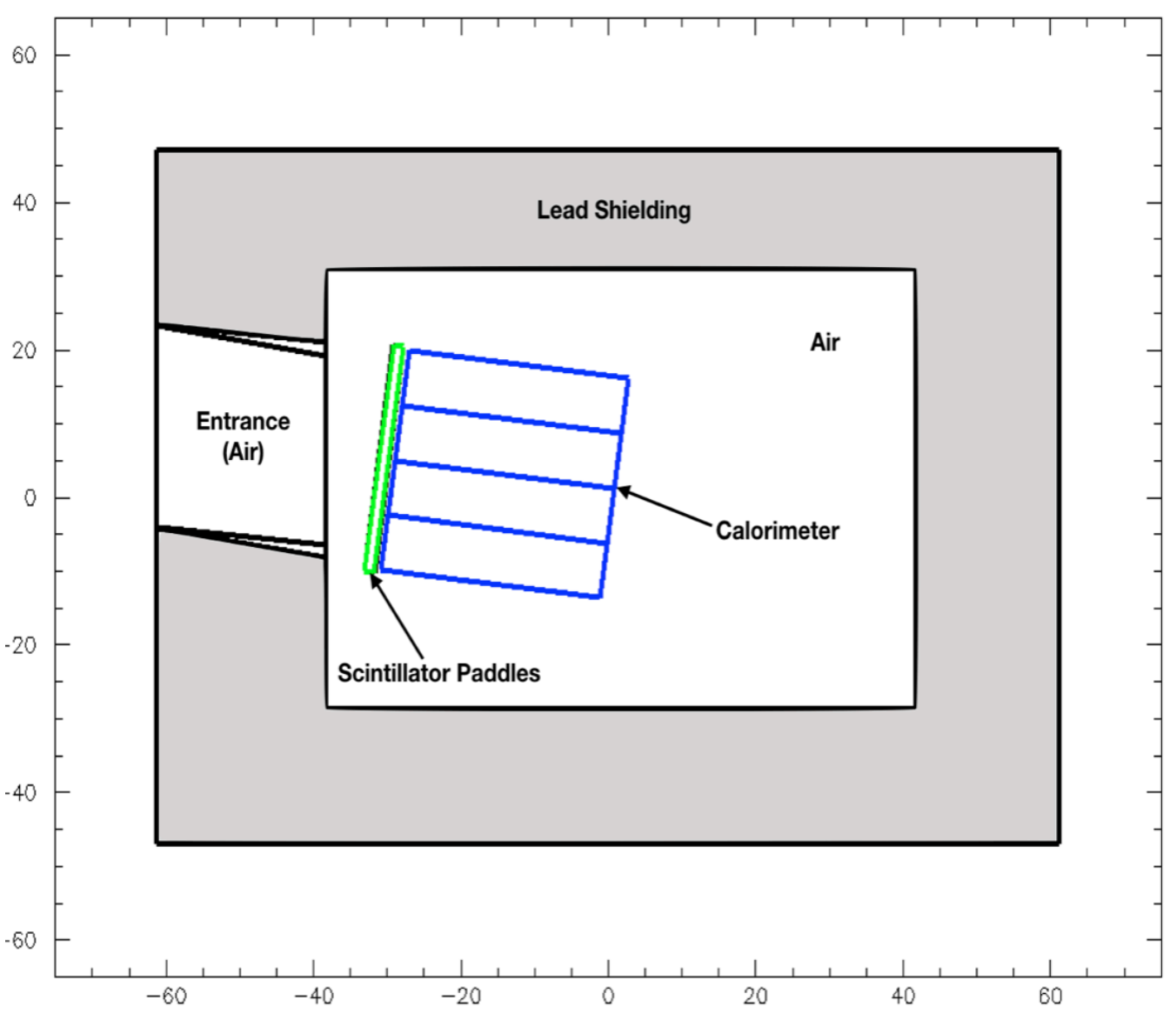

Figure 3.16: Detector Box in Geant 3 


\subsection{Geant3 Simulation}

\subsubsection{Physics Processes}

Various process can be turned on or off in the Geant 3 package. They include

- Decay in flight

- Multiple Scattering

- Nuclear Fission induced by a photon

- Muon-nucleus interactions

- Continuous Energy Loss

- Photoelectric Effect

- Compton Scattering

- Pair Production
- Bremsstrahlung

- Rayleigh Effect

- $\delta$-ray Production

- Positron Annihilation

- Hadronic Interactions

- Light Absorption

- Synchrotron Radiation

Simulations of the Møller polarimeter have all processes turned on but contributions from multiple scattering, energy loss, and synchrotron radiation dominate over the other processes.

In order to save computational time only Møller scattering events are generated. The user specifies the beam energy and the $\theta$ and $\phi$ range to generate events. Events in $\theta$ are generated by weighting them according to the Møller cross section as seen in Figure 3.17. Events are generated wide enough to cover the entire acceptance of the detector.

Other important parameters that may be entered into the simulation are beam position, beam spot size, collimator opening, electron beam momentum uncertainty $(\delta p / p)$, target thickness, and the target electron momentum. The effects of these parameters on rates and analyzing power will be discussed in section 4 .

\subsubsection{Statistical Error}

The simulation outputs the number of coincidence pairs of electrons that reached the detector and the mean analyzing power, $\left\langle A_{z z}\right\rangle$. The statistical error associated with each of these quantities is approximated by running the simulation many times with a different random seed each time. Figure 3.18 is a histogram of the number of coincidence pairs and $\left\langle A_{z z}\right\rangle$ which were calculated each time the simulation ran for 100k generated Møller pairs. For 100k events, the coincidence pairs were $17,670 \pm 120$ and $\left\langle A_{z z}\right\rangle=0.7386 \pm 0.0003$ approximately following counting statistics. This is a fractional uncertainty in $\left\langle A_{z z}\right\rangle$ of $0.04 \%$. For 


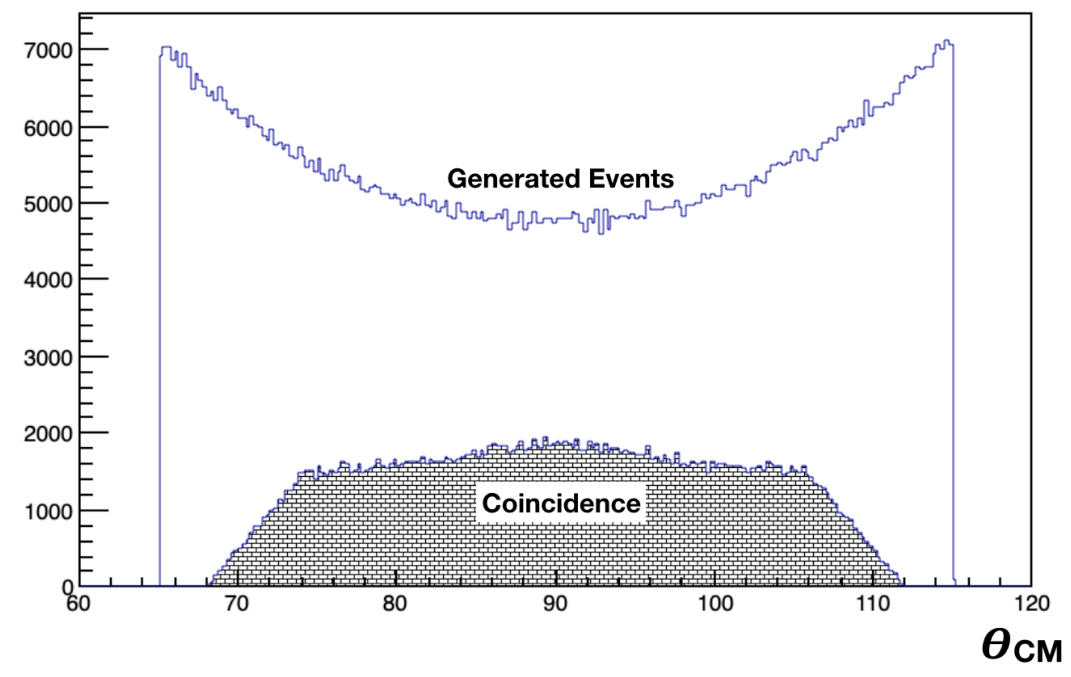

Figure 3.17: Geant3 Møller weighted generator for $65^{\circ} \leq \theta_{C M} \leq 115^{\circ}$. Electron pairs that were accepted by the detector are in the filled area labeled coincidence.

the systematic studies in Chapter 4, where high precision was required, 500k events were generated at each setting. 500k events gives a fractional uncertainty in $\left\langle A_{z z}\right\rangle$ of $\sim 0.015 \%$ as expected from counting statistics which is reflected in the error bar on the plots to follow. The error of the coincidence pairs, which are proportional to the rates in the detector, are $<1 \%$.

\subsubsection{Data Comparison}

In April of 2015 an optics scan of the Møller polarimeter was performed at a beam energy of $2.056 \mathrm{GeV}$. The current in three quadrupoles and the dipole were varied and data runs were taken at each setting. The data collected provided an opportunity to compare the results with simulation and the comparison can be seen in Figure 3.19. The general trend in rates between simulation and data agree. The spectrometer is tuned at $\mathrm{Q} 1=0.38 \mathrm{kG}, \mathrm{Q} 2=1.68 \mathrm{kG}$, $\mathrm{Q} 3=0.54 \mathrm{kG}$ and dipole $=3.03 \mathrm{kG}$. One can understand the fall off at lower quad current as events are no longer properly focused and fail to make it though the dipole aperture. These events are either clipped at the dipole entrance or hit the walls of the dipole vacuum box inside the dipole. The disagreement between the data and simulation at these lower fields is still being investigated. One attempt to explain the differences was to incorporate 

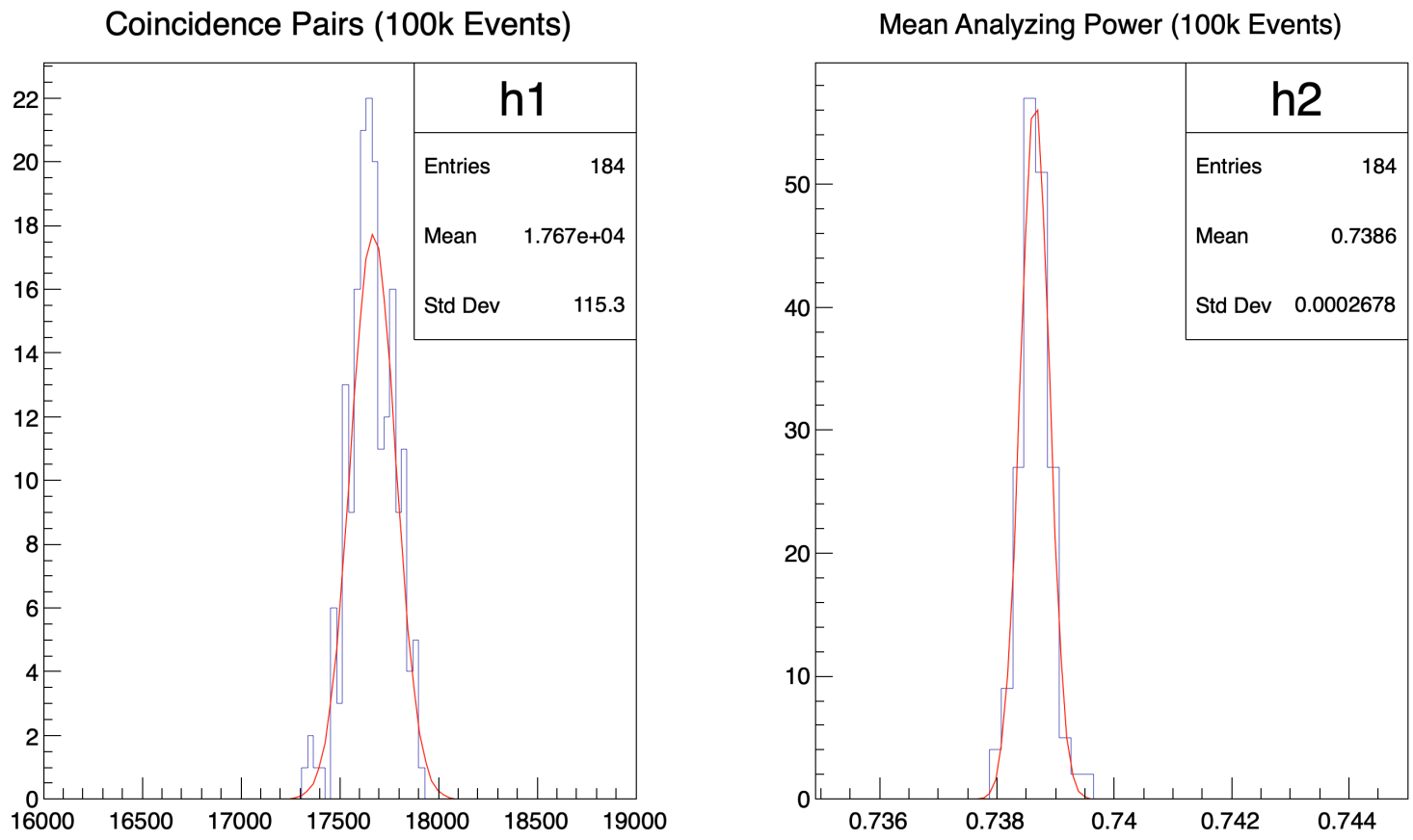

Figure 3.18: Coincidence pairs detected and $A_{z z}$ from simulation for 100k generated events. Each run of the simulation was done with a different random seed in order to approximate the statistical error. 
quadrupole field maps in GEANT4 which included higher multipole contributions to the field however this did not resolve the disagreement. The geometry in simulation was also carefully checked and when the possible the individual elements were measured to ensure an accurate model of the experimental setup.
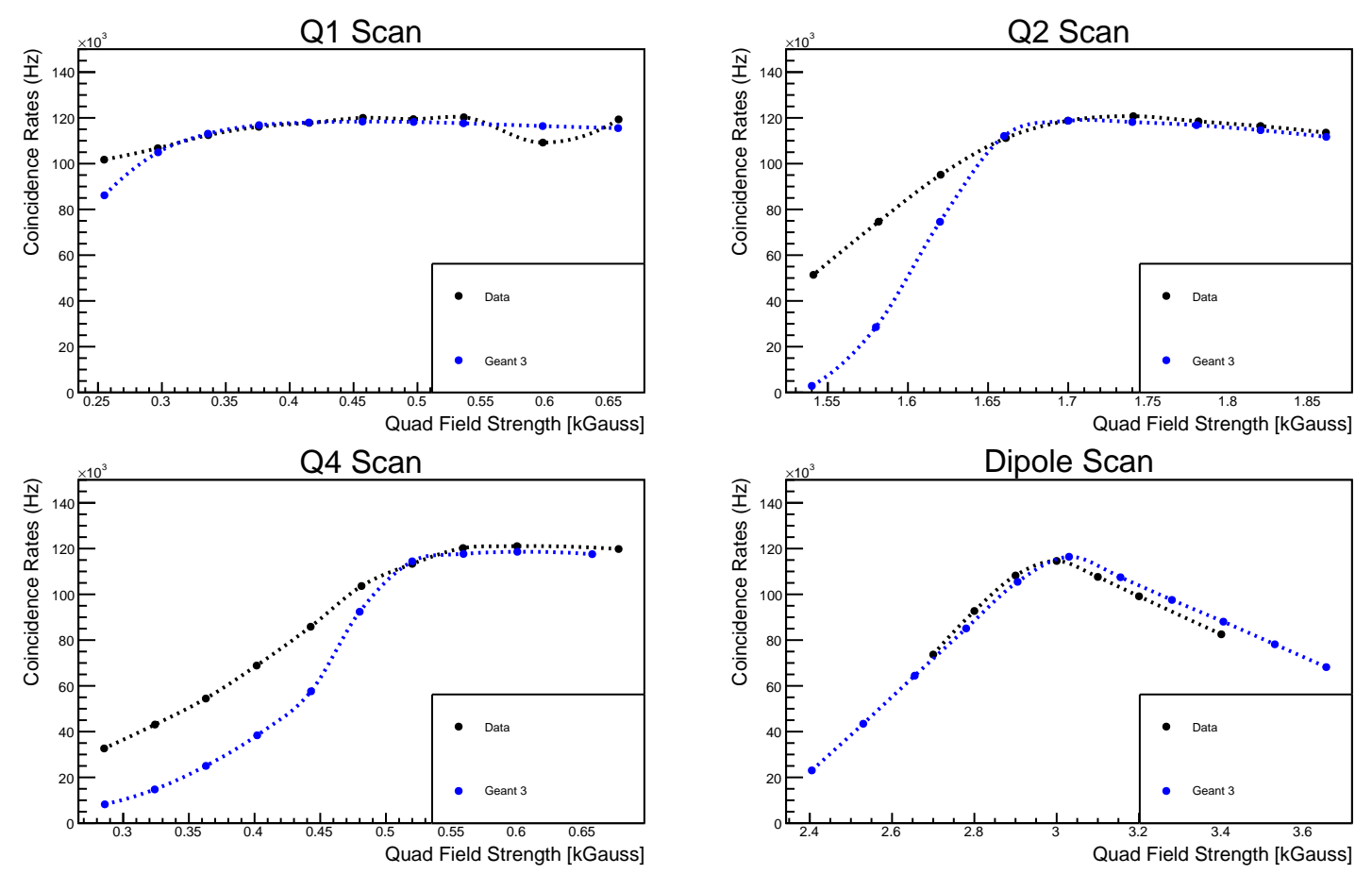

Figure 3.19: 2015 optics scan at $2.056 \mathrm{GeV}$ and Geant3 comparison (Q3 is turned off). Note that the single point in the $\mathrm{Q} 1$ scan at $0.6 \mathrm{kG}$ is suspect.

\subsubsection{Geant4 Comparison}

A Geant 4 simulation is currently under development in a collaboration with Syracuse University, Temple University and Jefferson Lab. Extensive comparisions between the everimproving Geant 4 simulation and Geant 3 simulations have been made which have allowed the Geant4 package to reach a mature state.

Initial comparisons made use of virtual planes placed at numerous places in the simulated polarimeter apparatus. This allowed the electrons to be tracked as they moved through the polarimeter. Figure 3.20 shows the electrons as they travel down the beam pipe after leaving the solenoid. Here we were concerned with how the target magnet twists the electrons as they leave the target area.. The upper image is from Geant 4 and the lower image 
is Geant3. Here the color scale is $\theta_{C M}$ (energy) and applies to the right hand side electrons (the left side is reversed so the "red" electrons on the left pair with a "red" on the right). Since Møller scattering involves two identical electrons, the simulation generates left and right pairs. In this simulation the tagged angle and energy belong to the right hand electron which always has a left-hand partner whose energy and angle are a precise function of the right hand electron. After good visual agreement was made (which was not the case at first), we did a numerical study. This was accomplished by ray tracing where we generated events at a specific $\theta$ and $\phi$ angle and recorded the (x,y) as they crossed the virtual planes. Figure 3.21 shows the positions of ten rays compared after the target magnet. The differences between the positions in the two simulations were found to be $<50 \mu \mathrm{m}$ in the quadrupole region and at $100 \mu \mathrm{m}$ at the dipole exit. These results are shown in 3.22. The spatial agreement between the simulations is smaller than the uncertainty in position of any component (magnet, detector, collimator) therefore at the level we are concerned with.

The most important requirement for the simulation is to provide a precise value for $\left\langle A_{z z}\right\rangle$. A comparison between Geant 4 and Geant 3 was made for a beam energy of $2.2 \mathrm{GeV}$ and an optics tune developed for CREX. Figure 3.23 shows the difference in $\left\langle A_{z z}\right\rangle$ between the simulation is $0.03 \%$. 


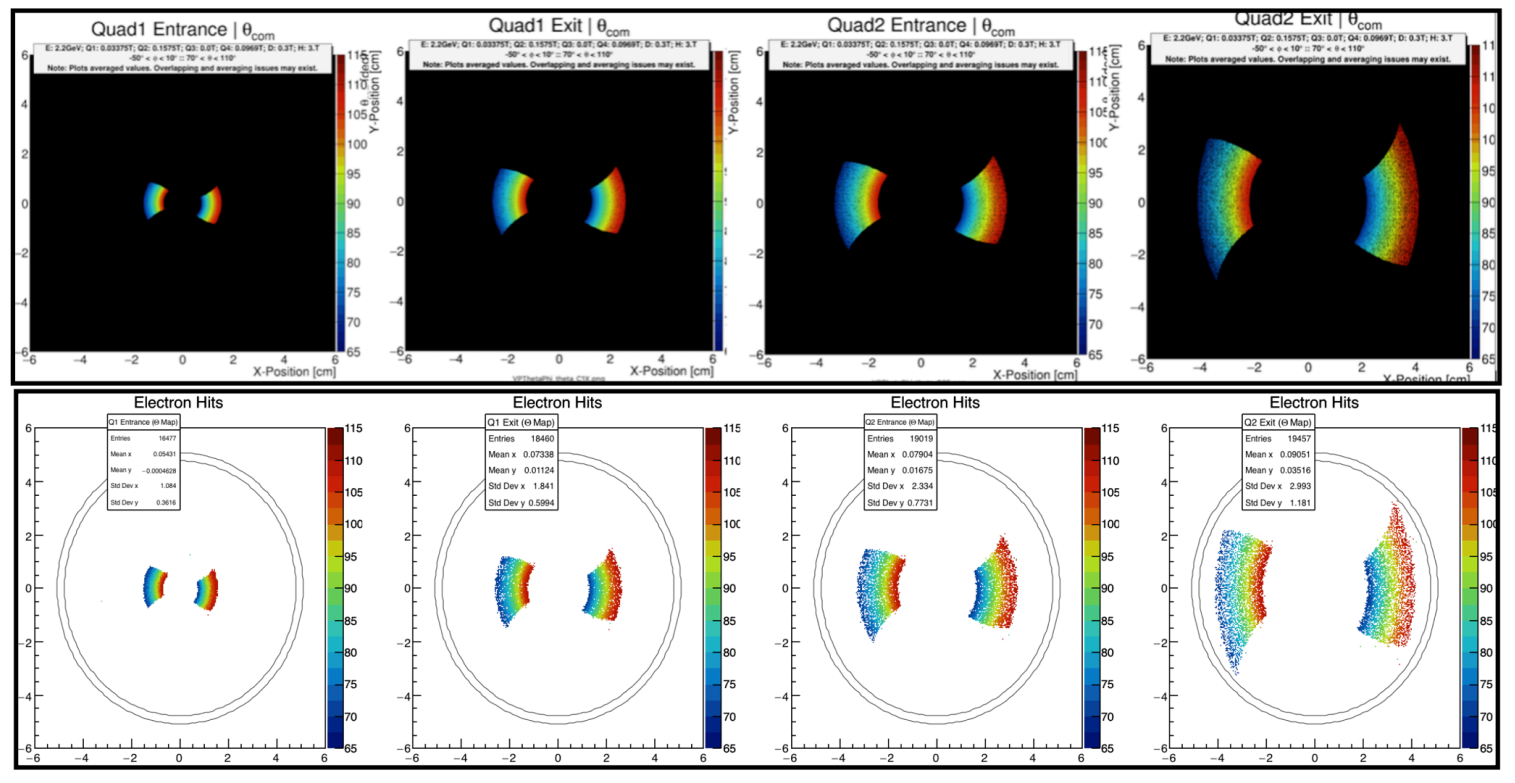

Figure 3.20: Geant3(bottom)/Geant4(top) Comparison. Tracking the electrons through the quadrupole region of the beam pipe. Color scale is $\theta_{C M}$ for RHS electrons 
Position at solenoid exit with ideal 3T holding field

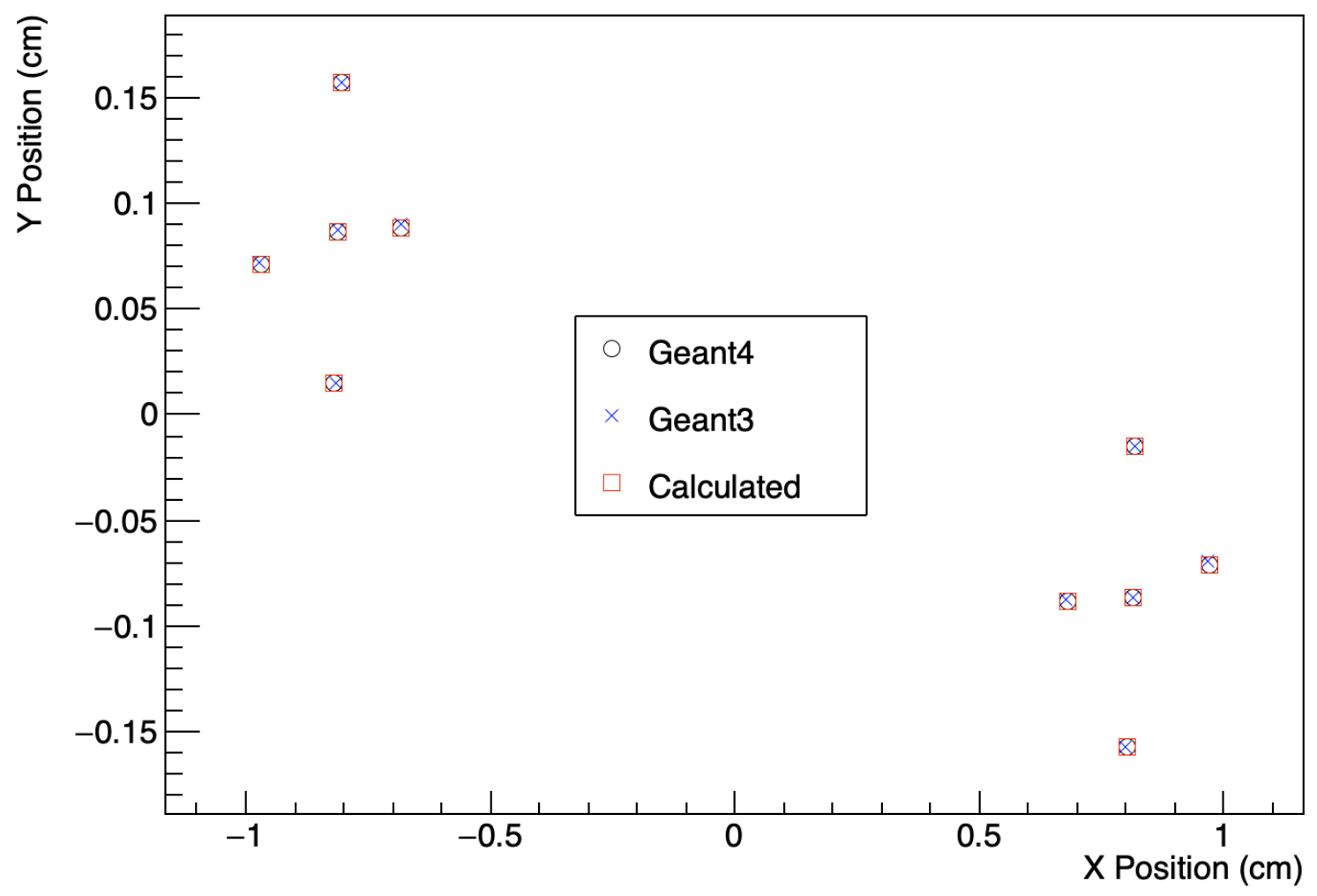

Figure 3.21: GEANT3 vs GEANT4 ray tracing comparison at split coil target magnet exit. 


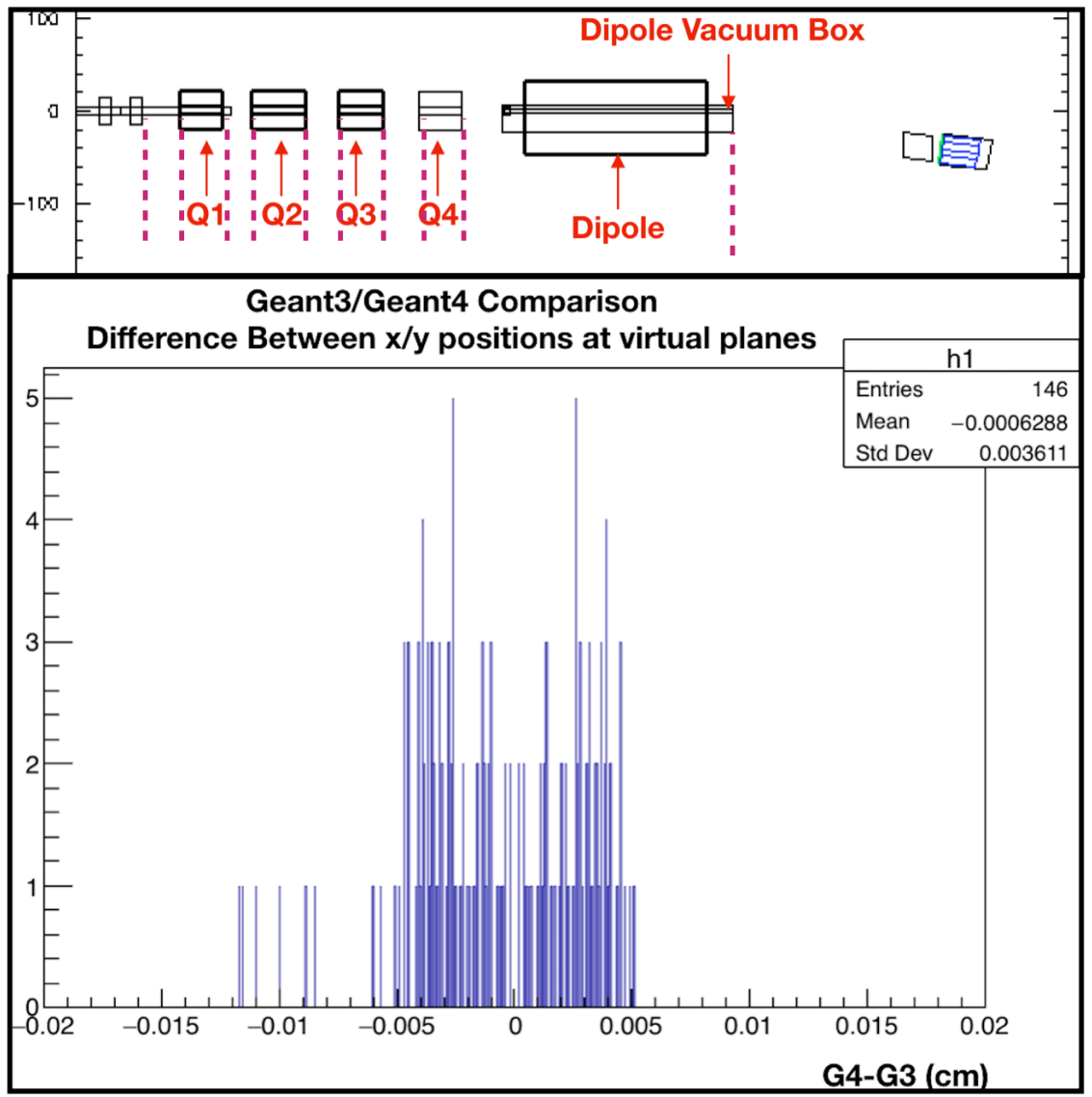

Figure 3.22: Geant3(bottom)/Geant4(top) ray tracing comparison. (Top)Magneta dashed lines indicate locations of virtual planes. (Bottom) Difference in position at planes between two simulations 


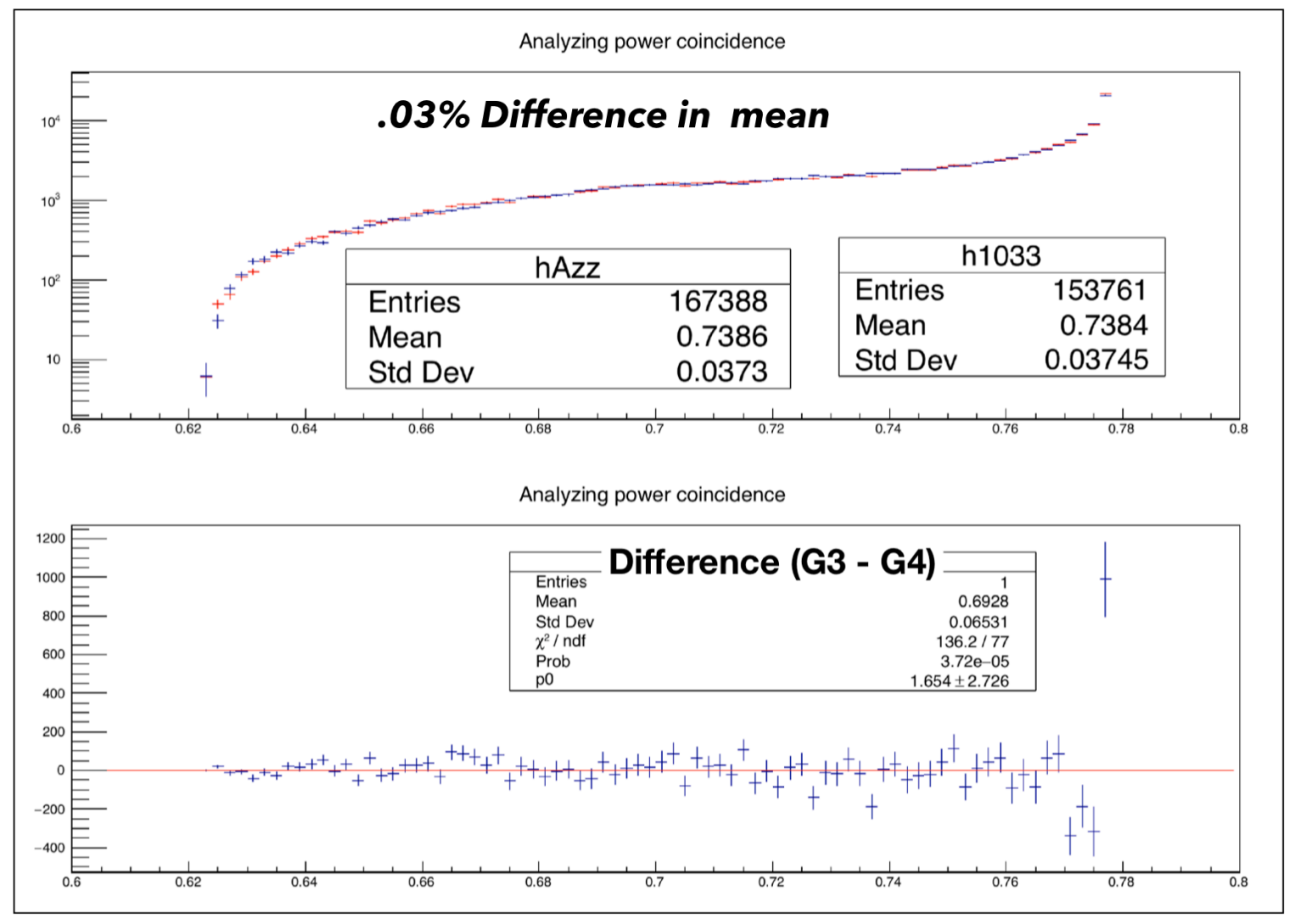

Figure 3.23: Geant3(bottom)/Geant4(top) Comparison. (Top) $A_{z z}$ at beam energy of $2.2 \mathrm{GeV}$. (Bottom) Difference in number of electrons in each $A_{z z}$ bin. 


\section{CHAPTER 4}

\section{UNCERTAINITIES IN MØLLER POLARIMETRY}

The systematic and statistical errors that contribute to the overall uncertainty in the final result of Hall A Møller polarimeter will be the focus of this chapter. Since the beam polarization we are measuring is

$$
P^{\text {beam }}=\frac{A}{P^{\text {foil }} \cdot\left\langle A_{z z}\right\rangle}
$$

the uncertainties in the measured asymmetry, $A$, the target polarization, $P^{f o i l}$ and the mean analyzing power $\left\langle A_{z} z\right\rangle$ can be found individually and then added in quadrature to obtain our final result $\frac{\delta P}{P}$. The error budget table for the MOLLER experiment, which is the most challenging near-term goal, is shown in Table 4.1. The statistical error in the measured asymmetry is the easiest to reduce by taking long enough data runs. Dead time and false asymmetry corrections will also effect the measured asymmetry and will be described and evaluated. However, the target polarization and analyzing power ultimately drive the magnitude of the final overall uncertainty. The precision to which we know the target polarization will be discussed and factors that contribute to it, such as target heating, angular momentum versus spin contributions, target angle and high field corrections. The systematic error in the analyzing power will be analyzed with Geant simulations. To determine $\frac{\delta A_{z z}}{A_{z z}}$, the sensitivities of all factors that affect the acceptance of the detector must be studied. These include contributions from beam position, quadrupole field strengths, and any uncertainty in the geometry of the experimental set-up that could contribute to a change in the analyzing power. Finally the Levchuck correction, a correction stemming from the 
Table 4.1: Error budget from MIE proposal to DOE for MOLLER experiment [17]

\begin{tabular}{||lcc||}
\hline Systematic Effect & Proposed Error & Strategic Approach \\
\hline \hline Target Polarization & $0.25 \%$ & Demonstrate saturation vs B and tilt angle \\
\hline Analyzing Power & $0.20 \%$ & Accurate spectrometer simulation \\
\hline Levchuk Effect & $0.20 \%$ & Simulation with atomic modeling \\
\hline Target Heating & $0.05 \%$ & Match data to heating calculation \\
\hline Deadtime & $0.10 \%$ & Confirm “zero dead time" w/ FADC \\
\hline Background & $0.10 \%$ & Measurements with beam \\
\hline Others & $0.10 \%$ & see text \\
\hline \hline Total & $0.42 \%$ & \\
\hline \hline
\end{tabular}

non-neglible energies of unpolarized bound inner shell electrons, which will also contribute to $\frac{\delta P}{P}$ the will be presented and the methods used to calculate it.

\subsection{Statistical Errors}

Although Møller polarimetry uncertainties are dominated by systematic errors, I will start by addressing the statistical errors in an asymmetry measurement.

The measured Møller scattering asymmetry, $A$, is defined as

$$
A=\frac{N_{+}-N_{-}}{N_{+}+N_{-}}
$$

where $N_{+}$and $N_{-}$are the number of events for the + and - helicity states respectively in one asymmetry measurement. The beam helicity is flipped at a fixed rate and the total number of events in a given state is summed. That is N. Typically an asymmetry is measured in what is termed a "quartet pattern" where two + and two - states are included in either a -++- or a +-+ pattern. This pattern guarantees the cancellation of any first order drifts in time. The uncertainty, $\delta A$, in one measurement is

$$
\delta A^{2}=\left(\frac{\partial A}{\partial N_{+}} \delta N_{+}\right)^{2}+\left(\frac{\partial A}{\partial N_{-}} \delta N_{-}\right)^{2}
$$

Since

$$
\frac{\partial A}{\partial N_{+}}=\frac{2 N_{-}}{\left(N_{+}+N_{-}\right)^{2}} \text { and } \frac{\partial A}{\partial N_{-}}=\frac{-2 N_{+}}{\left(N_{+}+N_{-}\right)^{2}}
$$


Equation 4.3 becomes

$$
\delta A^{2}=\frac{4}{\left(N_{+}+N_{-}\right)^{4}}\left(N_{-}^{2} \delta N_{+}^{2}+N_{+}^{2} \delta N_{-}^{2}\right)
$$

If we let $N_{s}$ be the total number of events in a single asymmetry measurement

$$
N_{s}=N_{+}+N_{-}
$$

and assume $\delta N_{ \pm}^{2}=N_{ \pm}$then the uncertainty becomes

$$
\delta A^{2}=\frac{4}{N_{s}^{4}}\left(N_{-}^{2} N_{+}+N_{+}^{2} N_{-}\right)
$$

It should be noted that the assumption $\delta N_{ \pm}^{2}=N_{ \pm}$only holds if the variance of $N_{ \pm}$ follows ordinary Poisson statistics. To test this assumption I will show that the outcome of this statistical approximation agrees with experimental data.

We can eliminate $N_{+}$and $N_{-}$using equations 4.2 and 4.6 and deriving the relations

$$
\begin{gathered}
N_{-}=\left(\frac{1-A}{1+A}\right) N_{+}=\alpha N_{+} \\
N_{+}=\frac{N_{s}}{1+\alpha}
\end{gathered}
$$

where $\alpha=\frac{1-A}{1+A}$. Equation 4.7 can be rewritten as

$$
\delta A^{2}=\frac{4}{N_{s}^{4}}\left(\alpha N_{+}^{3}+\alpha^{2} N_{+}^{3}\right)=\frac{4}{N_{s}^{4}}\left(\frac{\alpha}{(1+\alpha)^{3}} N_{s}^{3}+\frac{\alpha^{2}}{(1+\alpha)^{3}} N_{s}^{3}\right)=\frac{4 \alpha}{(1+\alpha)^{2}} \frac{1}{N_{s}}
$$

For small asymmetries $\alpha \approx 1$. A typical value of $A$ for a polarimetry run is 0.055 which corresponds to $\alpha=0.896$ and $\frac{4 \alpha}{(1+\alpha)^{2}}=0.997$. Therefore we can neglect the factor with $\alpha$ and write the uncertainty simply as

$$
\delta A \approx \frac{1}{\sqrt{N_{S}}}
$$

To check this result we can apply equation 4.11 to data. Figure 4.1 plots the measured asymmetry for run \#16159 and figure 4.2 is a tabulated analysis of the same run. The run was 3 minutes and 55 seconds long. Inside the red boxed area are the coincidence and accidental rates measured in rate/cycle where cycle refers to a single helicity state window. 
The accidental rate accounts for coincidences that occur that are not from a single Møller scattering event. The accidental rate is determined by delaying the signal in one arm of the detector and comparing the delayed signal to the original non-delayed signal to see how many coincidences are found. Since one asymmetry measurement consists of four cycles (two $N_{+}$and two $N_{-}$) we should multiply those numbers by four to get the total number of events in one helicity quartet. Putting this into equation 4.11 gives

$$
\delta A=\frac{1}{\sqrt{4(3099.7)-4(33.1)}}=0.009029
$$

The $\sigma$ of the gaussian fit in figure 4.1 is 0.009095 . Equation 4.11 gives a better than $1.0 \%$ agreement with data and therefore we can conclude that the statistical uncertainties are dominated by normal counting statistics.

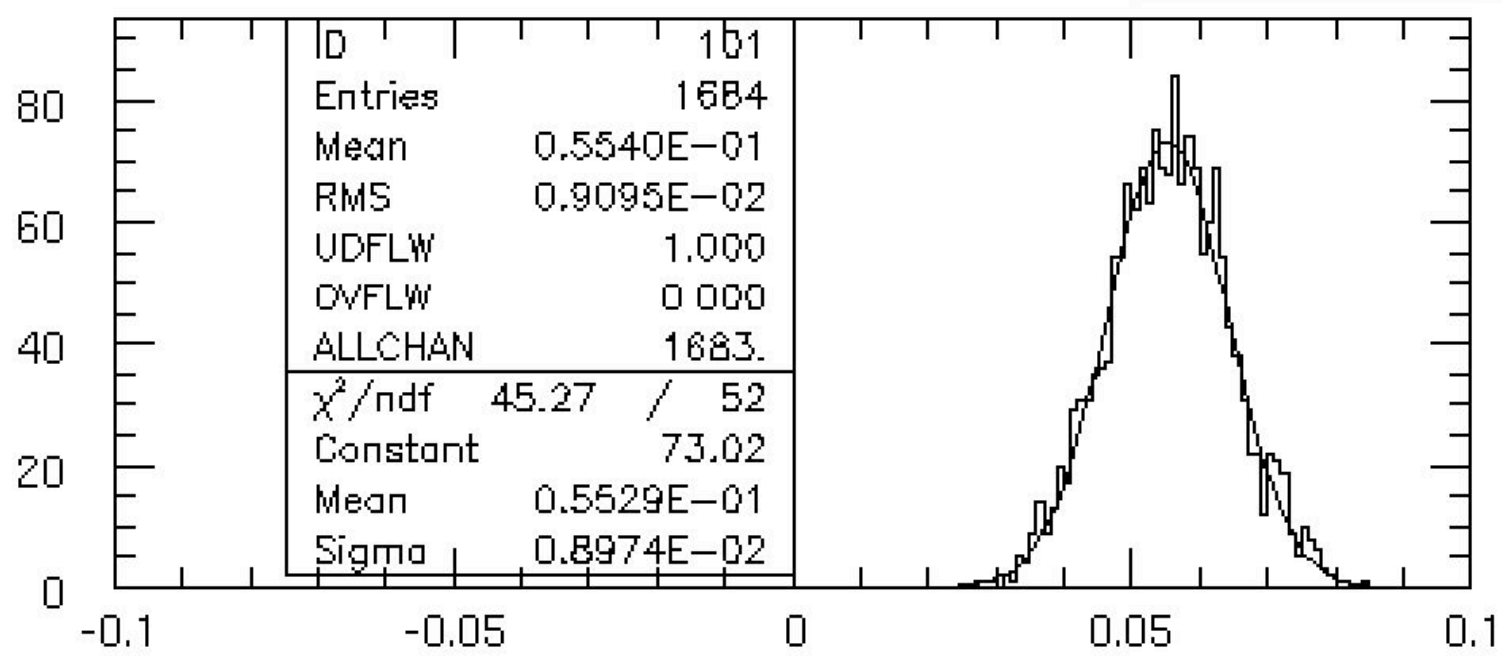

Figure 4.1: Asymmetry measurement for run 16159. Each entry corresponds to one quartet or two $N_{+}$and two $N_{-}$cycles

The uncertainty after $N_{c}$ measurements, or the standard deviation of the mean, $\sigma_{M}$ is

$$
\sigma_{M}=\frac{\delta A}{\sqrt{N_{c}}}=\frac{1}{\sqrt{N_{c} N_{s}}}=\frac{1}{\sqrt{N}}
$$

Where we let $N$ be the total number of events in the run. Run \#16159 consisted of 1684 measurements, applying equation 4.13

$$
\sigma_{M}=\frac{\delta A}{\sqrt{N_{c}}}=\frac{0.009}{\sqrt{1684}}=2.2 \times 10^{-4} ;
$$




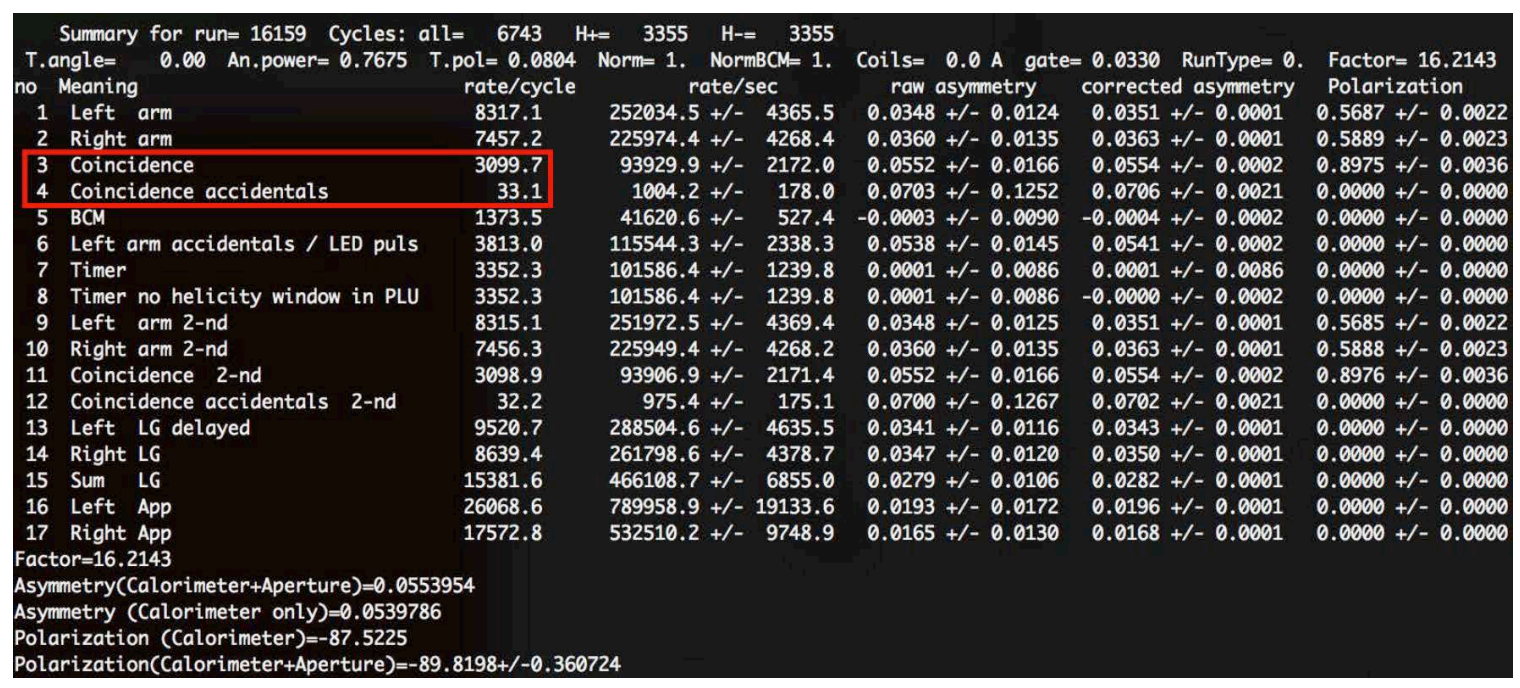

Figure 4.2: Output of analysis script for run 16159

The fractional uncertainty in the final result is

$$
\frac{\sigma_{M}}{\langle A\rangle}=\frac{2.2 \times 10^{-4}}{5.5 \times 10-2}=0.40 \%
$$

The coincidence rate $r$, the number of events $N$ and time $t$ are simply related by

$$
N=r t
$$

Using equations 4.13 and 4.16 we can determine the time required to reach a certain precision as a function of rate.

$$
t=\frac{1}{\langle A\rangle^{2} r\left(\frac{\sigma_{M}}{\langle A\rangle}\right)^{2}}
$$

Figure 4.3 shows equation 4.17 plotted for three different values of the relative uncertainty. Rearranging equation 4.17 allows us to plot the statistical error as a function of time and this is shown in figure 4.4 for three different coincidence rates.

From figures 4.3 and 4.4 we see that a statistical fractional uncertainty of $0.5 \%$ can be reached in less than five minutes for rates greater than $40 \mathrm{kHz}$. To reach $0.2 \%$, a data run could be from 15 - 30 minutes depending on the coincidence rates. For experiments requiring a statistical uncertainty of less than $0.1 \%$ the data runs may be as long as 90 minutes ( $60 \mathrm{~Hz}$ rate) but with a higher rate could be as short as 60 minutes ( $90 \mathrm{~Hz}$ rate). To give the reader an idea of the typical rates during a Møller run, figure 4.5 shows the 
Time to reach desired precision as a function of coincidence rate

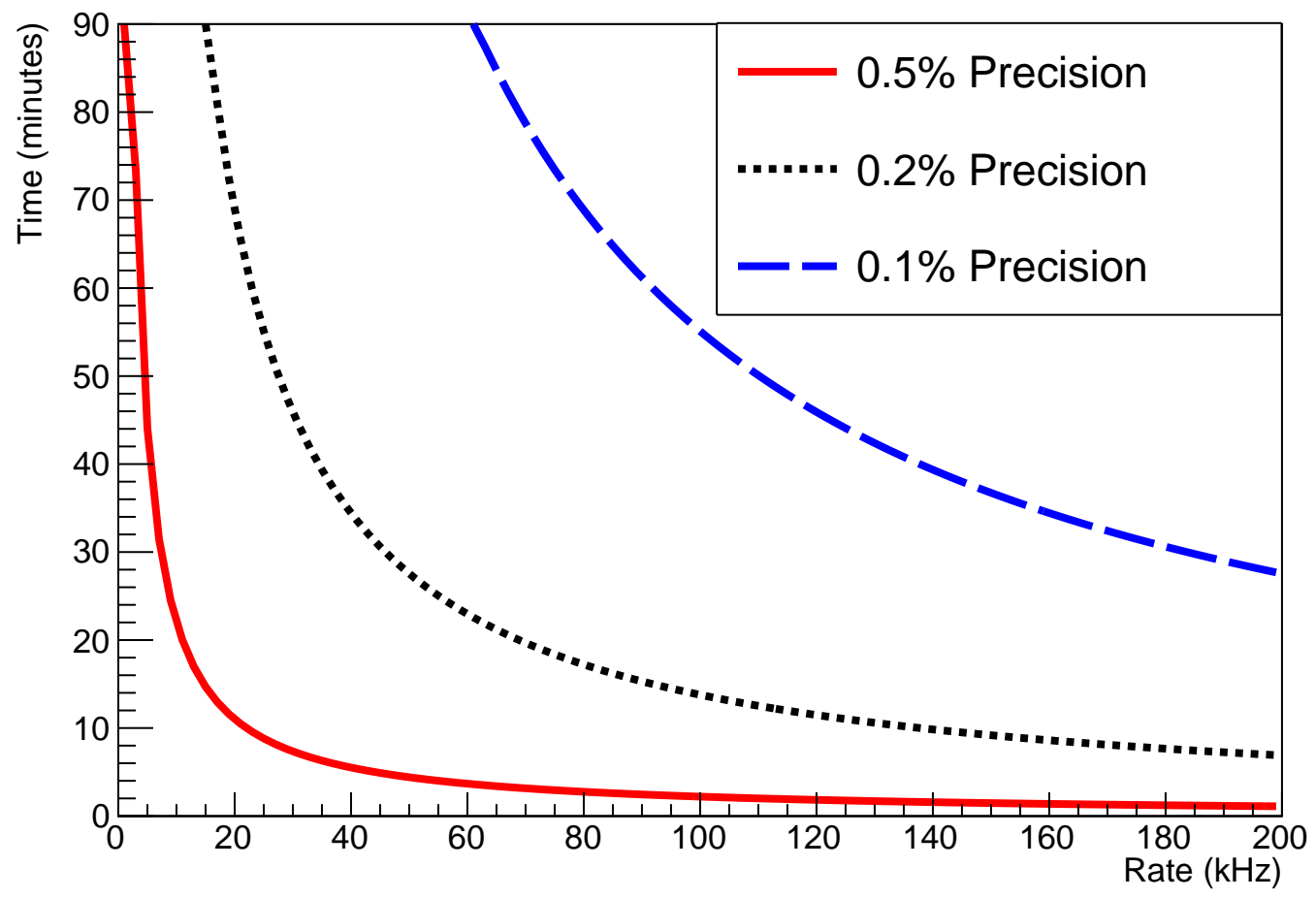

Figure 4.3: Time required to reach level of relative uncertainty versus coincidence rate. 
Time required to reach desired precision for various coincidence rates

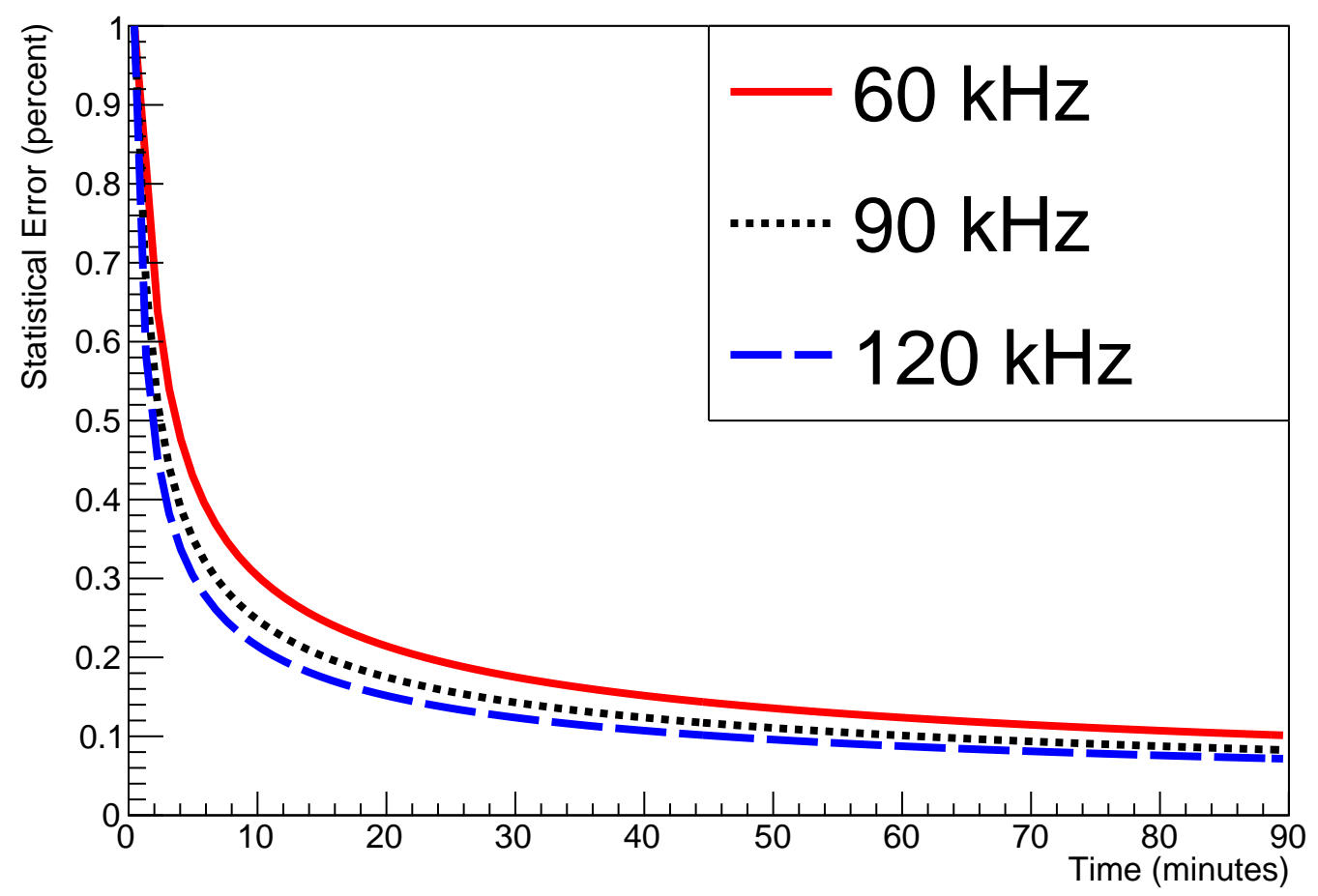

Figure 4.4: Relative statistical uncertainty versus length of run for various coincidence rates. 


\section{Coincidence rates in 2016}

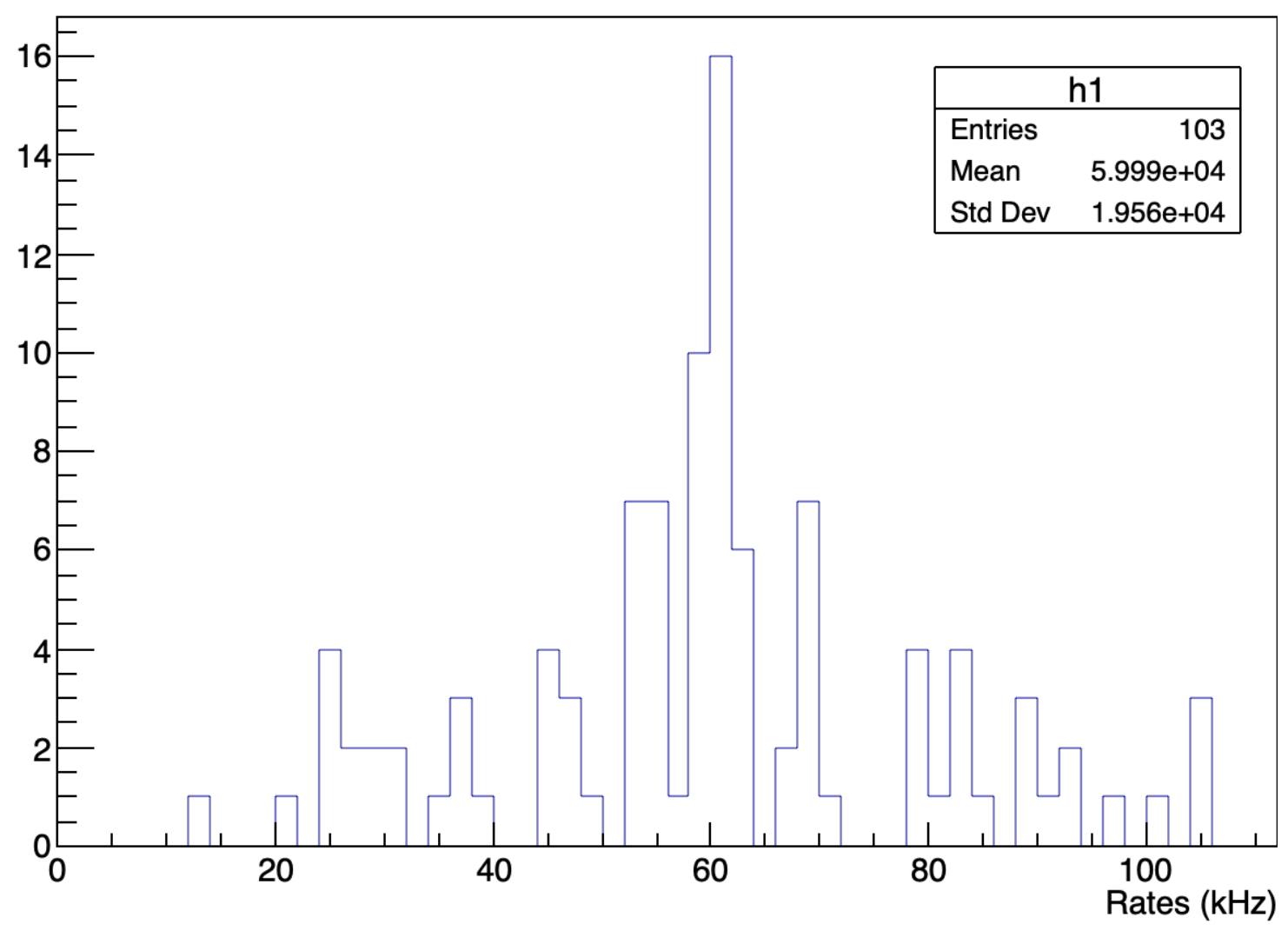

Figure 4.5: Coincidence rates for various runs in 2016 during DVCS 
coincidence rates for a number different runs in 2016 for beam energies from $4.4 \mathrm{GeV}$ to $11 \mathrm{GeV}$.

\subsection{Dead Time Corrections}

After a data acquisition (DAQ) system registers an event, it is incapable of recording another event for a short period of time. This is referred to as the dead time, $\tau$. As a result of the dead time, the measured rates are smaller than the actual rates and a correction needs to be applied. The probability that an event will occur during the dead time, assuming Poisson statistics, is $1-e^{-R \tau}$ for a given rate, $R$. Therefore the measured rate is [60]

$$
R_{M}=R\left(1-\left(1-e^{-R \tau}\right)\right)
$$

In the limit of $R \tau \ll 1$, which is true for these measurements as we will see, Equation 4.18 becomes

$$
R_{M}=R(1-R \tau)
$$

This result allows us to write the rates for the two different helicity states as

$$
R_{M}^{ \pm}=R^{ \pm}\left(1-R^{ \pm} \tau\right)
$$

The fact that the dead time correction factor $R \tau$ depends on rates is essential, since it results in different correction factors for different helicity states. Therefore, when we form the asymmetry by taking the ratio of rates, this factor doesn't cancel out:

$$
A_{M}=\frac{R_{m}^{+}-R_{m}^{-}}{R_{m}^{+}+R_{m}^{-}} \simeq A(1-R \tau),
$$

where $A_{m}$ and $A$ are the measured and the physical asymmetries respectively. As we can see, dead time goes into the asymmetry directly as a first order correction, so it is an important systematic effect and needs to be understood thoroughly. [60]

In 2010 , the dead time of the discriminator was measured to be $\sim 20 \mathrm{~ns}$ [61]. The measurement was made by sending a train of two pulses from a pulse generator into the discriminator. The time between the two pulses was reduced until the discriminator only output one pulse. The dead time of the programable logic unit (PLU) was measured in a similar fashion and was found to have a negligible dead time. These measurements give us 
insight to the approximate size of the dead time correction we can expect in a typical measurement, $(20 \mathrm{~ns})(100 \mathrm{kHz})=0.2 \%$. However dedicated runs are usually taken to measure the dead time correction using LEDs that are installed in both arms of the calorimeter. The LED's emit light at a known frequency and the pulses are counted as the beam current is varied. As the current, therefore the rates, are increased the dead time correction also increases. This can be seen in Figure 4.6. The slope of the fit gives the dead time of the system, here $16.8 \mathrm{~ns}$. The uncertainty in the correction can be found by the error in the fit of the slope, for this run it was $0.03 \%$. Therefore a reasonable uncertainty to assign in the dead time correction is $0.05 \%$.

\section{Dead time correction vs Rates}

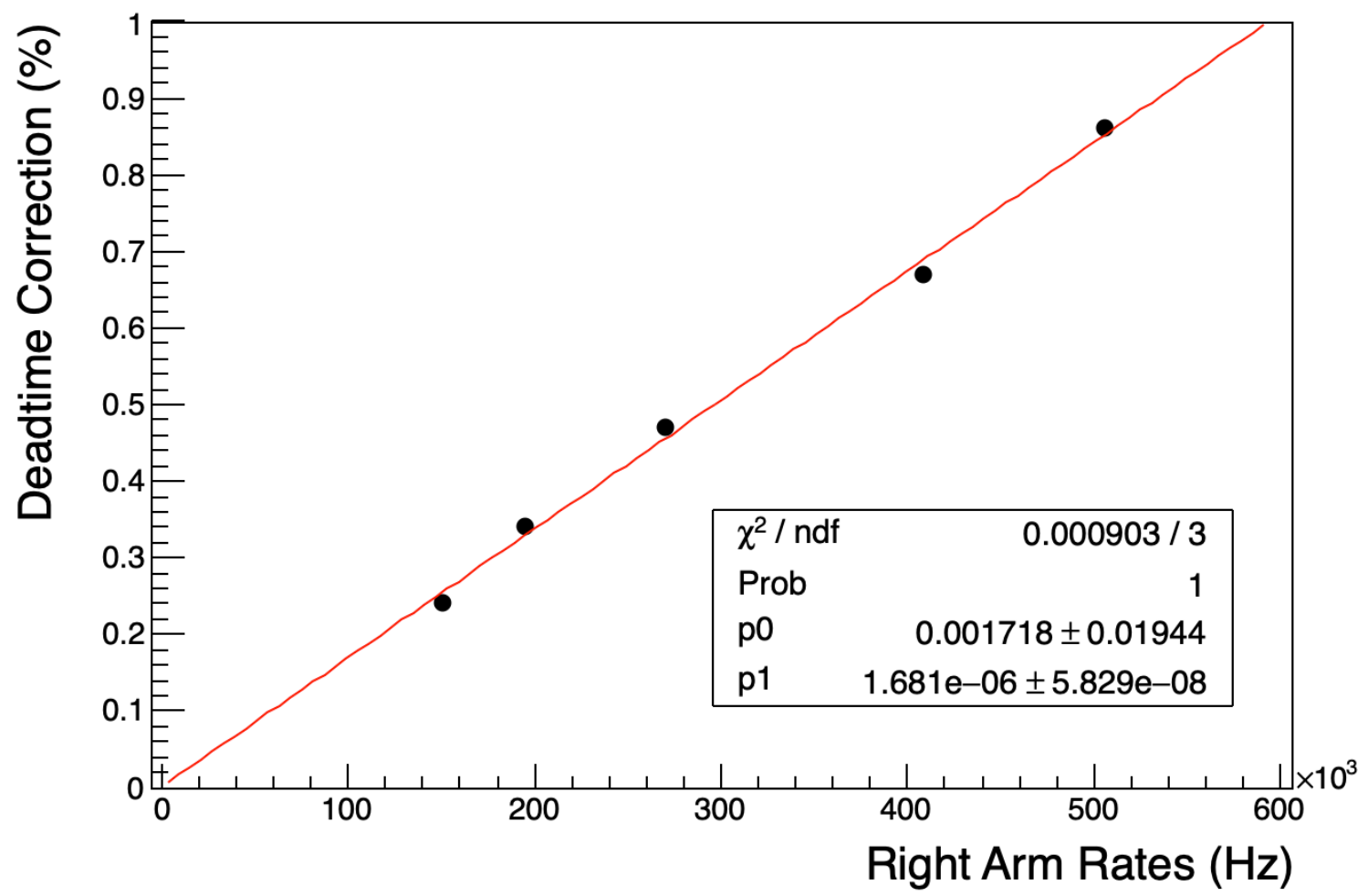

Figure 4.6: Data from dedicated LED pulser runs to determine dead time correction 


\subsection{False Asymmetries}

When performing an asymmetry measurement an experimentalist must be aware of how to treat false asymmetries. In experiments where asymmetries are constructed from helicity flipping in a polarized beam one has to be certain that it is only the helicity that is changing but in practice this can be very difficult to control. For instance the beam's position, angle, energy and current could change when the helicity is flipped which would lead to a helicity correlated beam asymmetry. For precision parity violation experiments, even the presence of the electronic logic signal that indicates the helicity state of the beam into the DAQ can introduce a large false asymmetry and for this reason the signal is often delayed several cycles to cancel out the effect.

Suppose you have two identical experiments for which you are performing an asymmetry measurement. In the first experiment there are no helicity correlated differences in the beam and you measure an asymmetry $A$. In the second experiment suppose the beam position's or current is changing between helicity states which cause an additional difference in the measured rates and you measure an asymmetry $A^{\prime}$. Let $N_{ \pm}$be the actual difference in counting rates from the physics process being measured and $\delta$ be the difference in rates due to the helicity correlated beam position or current. We can write $A^{\prime}$ as

$$
A^{\prime}=\frac{\left(N_{+}+\frac{\delta}{2}\right)-\left(N_{-}-\frac{\delta}{2}\right)}{\left(N_{+}+\frac{\delta}{2}\right)+\left(N_{-}-\frac{\delta}{2}\right)}=A+\frac{\delta}{N_{+}+N_{-}}=A+\frac{\delta}{\bar{N}}
$$

Where $\bar{N}$ is the average rate. As an example suppose the beam position varied by $0.2 \mathrm{~mm}$ between helicity states. From Figure 4.27 we can see that a $0.2 \mathrm{~mm}$ shift in beam position can lead to a change of in rates of $1 \%$. A helicity correlated beam position difference of $0.2 \mathrm{~mm}$ would lead to a $1.0 \%$ false asymmetry correction that would need to be accounted for in the final result. A helicity correlated beam position of this size is not likely, because the position differences for parity experiments are expected to be controlled at the submicron level near the target in the Hall (see pages 241-242 of [7]) but one can imagine how multiple smaller helicity correlated variables could effect the overall measured asymmetry. The current plan to address false asymmetries is to use a copper foil in the target ladder to explicitly measure the "null asymmetry" and bound all sources of false asymmetries. 
Table 4.2: Foil polarization from [14]

\begin{tabular}{||lccc||}
\hline Effect & $M_{S}\left[\mu_{B}\right]$ & Error & Ref. \\
\hline \hline Spontaneous Magnetization $(T \rightarrow 0, \mathrm{~B} \rightarrow 0)$ & 2.2160 & 0.0008 & {$[62]$} \\
\hline Saturation Magnetization $(T=294 \mathrm{~K}, \mathrm{~B}=1 \mathrm{~T})$ & 2.177 & 0.002 & {$[63]$} \\
\hline High Field Corrections & 0.0059 & 0.002 & {$[15]$} \\
\hline Orbital contribution (using g') & 0.0918 & 0.0033 & {$[64]$} \\
\hline
\end{tabular}

\subsection{Target Polarization}

Future demands of Moller polarimetry at JLab requires beam polarization measurements with a precision of better than $0.5 \%$ and the precision that which we know the target polarization plays a crucial role. We strive to know this value, which is the fraction of electrons that contribute to the asymmetry, to better than $0.25 \%$. The value frequently cited in the polarimetry literature is $0.08043 \pm 0.00015( \pm 0.19 \%)$. This meets our requirements on precision, however the value is taken from a 1997 NIM paper by deBever et al.[14], which includes references and sources dating as far back as the 1920's and overlooks some approximations that add an additional uncertainty to the final result. To follow is a summary of the path taken to reach the cited value while bringing attention to some concerns that exist. I will also examine newer methods and more recent findings.

The foil polarization value is the fraction of electrons contributing to the observed asymmetry in Moller scattering. The polarization is found from the magnetization which changes with different applied fields and temperatures and the final polarization is also determined by splitting the total magnetization into its spin and orbital contributions. In Bever's 1997 NIM paper[14], there is a section which explains how and where the different values are found. Here they are tabulated:

The first line of the table is the saturation magnetization in the limit of temperature and external field going to 0 , also referred as the spontaneous saturation. The value is from a 1968 paper[62] which calculates the mean value from five other works: Weiss and Forrer(1929), Peschard(1925), Pauthenet(1952), Danan(1958), and Meyer and Herr(1966). In all these works the magnetization was measured at temperatures exceeding $0 \mathrm{~K}$ and extrapolated to $0 \mathrm{~K}$. The experimental method used was the Weiss and Forrer axial extraction, also called the ballistic method [65], which places small bulk samples in a known applied 
magnetic field (up to 3T) and then the sample is suddenly extracted and the change in flux is measured.

In 1971 Crangle and Goodman published The Magnetization of Pure Iron and Nickel[13] which included results from their own measurements of magnetization on small bulk iron samples. Instead of using the axial extraction method they used a force method. They placed the samples in stong magnetic field gradients and measured the force exerted by the sample. Their results support the accuracy of the previous works for the magnetization at $0 \mathrm{~K}$ and $293 \mathrm{~K}$. The paper also gives table of values for the spontaneous magnetization as a function of temperature from $0 \mathrm{~K}$ to $1023 \mathrm{~K}$. Crangle's and Goodman's results agree very well, but with much less precision with the Weiss and Forrer axial extraction method experiments (see Figure 4.8).

\begin{tabular}{|c|c|c|c|}
\hline \multicolumn{4}{|c|}{ Magnetization of pure iron and nickel } \\
\hline \multirow{2}{*}{\multicolumn{4}{|c|}{$\begin{array}{l}\text { TABLE 2. SUMMARY OF EXISTING ABSOLUTE MEASUREMENTS } \\
\text { ON THE MAGNETIZATION OF NICKEL AND IRON } \\
\text { Data for nickel below } 300 \mathrm{~K} \text { are derived from Kaul \& Thompson (i969). } \\
\text { Magnetization/emu g }{ }^{-1} \text {. }\end{array}$}} \\
\hline & & & \\
\hline & \multicolumn{3}{|c|}{ temperature/K } \\
\hline authors & 293 & 77 & 0 (or 4) \\
\hline \multicolumn{4}{|l|}{ nickel } \\
\hline Weiss \& Forrer (1929) & 54.3 & - & $\begin{array}{c}57.6 \\
\text { (extrapolated) }\end{array}$ \\
\hline Danan (1958) & 55.2 & - & - \\
\hline $\begin{array}{l}\text { Danan, Herr \& Meyer } \\
\text { (Ig68) }\end{array}$ & - & - & $58.57 \pm 0.03$ \\
\hline present work & $55.1 \pm 0.1$ & $58.4 \pm 0.1$ & $58.6 \pm 0.1$ \\
\hline \multicolumn{4}{|l|}{ Tron } \\
\hline Weiss \& Forrer (1929) & 217.61 & - & $\begin{array}{c}221.74 \\
\text { (extrapolated) }\end{array}$ \\
\hline $\begin{array}{l}\text { Danan (1958) (same } \\
\text { specimen as above) }\end{array}$ & 217.35 & - & - \\
\hline $\begin{array}{l}\text { Danan, Herr \& Meyer } \\
\text { (I968) }\end{array}$ & - & - & $221.71 \pm 0.08$ \\
\hline present work & $217.6 \pm 0.1$ & $221.4 \pm 0.1$ & $221.7 \pm 0.1$ \\
\hline
\end{tabular}

Figure 4.7: Magnetization of Fe from [13] using force method

Once the spontaneous magnetization is determined, it needs it to be corrected for higher temperatures which lowers the magnetization. The temperature dependence can be approximated by Bloch's Law [66]:

$$
M(T)=M(T=0)\left(1-a_{\frac{3}{2}} T^{\frac{3}{2}}\right)
$$


This equation is valid for temperatures far below the Curie temperature. For better agreement with experimental data, terms with higher orders of T may also be included and whose coefficients have been determined[67].

The temperature corrected saturation magnetization is given in the second line of the table, which is cited from a 1982 paper by C.D. Graham[63]. Graham states This paper collects and summarizes the available literature values for the room temperature saturation magnetization of nickel and iron in an attempt to decide the best values to use for calibrating equipment for magnetic measurements. His magentization data is shown in Figure 4.8.

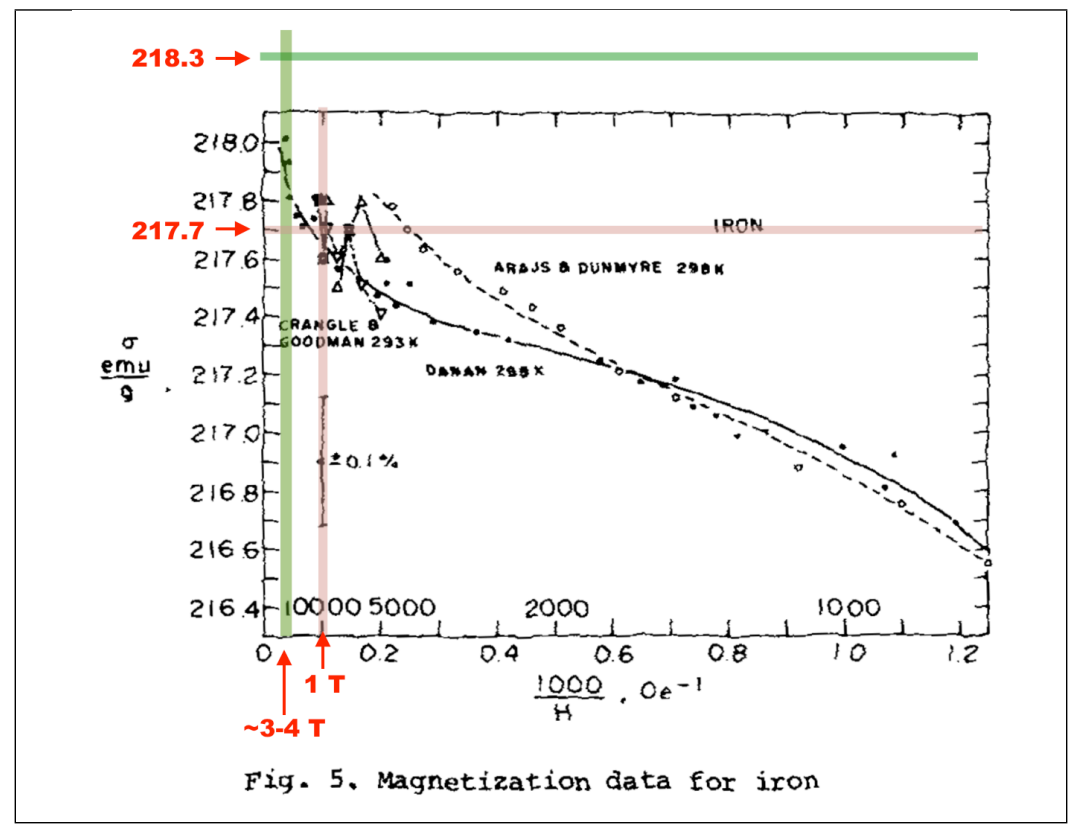

Figure 4.8: Iron magnetizations from Graham. Colored bars represent the values used in [14]

In addition to a temperature correction, the saturation magnetization needs to be adjusted for relatively high applied magnetic fields. The high field correction is listed in the third line of Table 4.2. At higher fields two correction terms are needed:

$$
M_{s}(B, T)=M_{s}(0, T)+a(T) \sqrt{B}+b(T) B .
$$

The term proportional to $\sqrt{B}$ accounts for spin waves while the term proportional to $\mathrm{B}$ describes the response of conduction electrons. The corrections are at the $0.5 \%$ level at 4 Tesla which translates to a $<0.01 \%$ uncertainity in the final target polarization value. The 
work cited is from Pauthenet's 1982 paper which studied spin waves along specific crystal axes[15]. His fit to experimental data is shown in Figure 4.9.

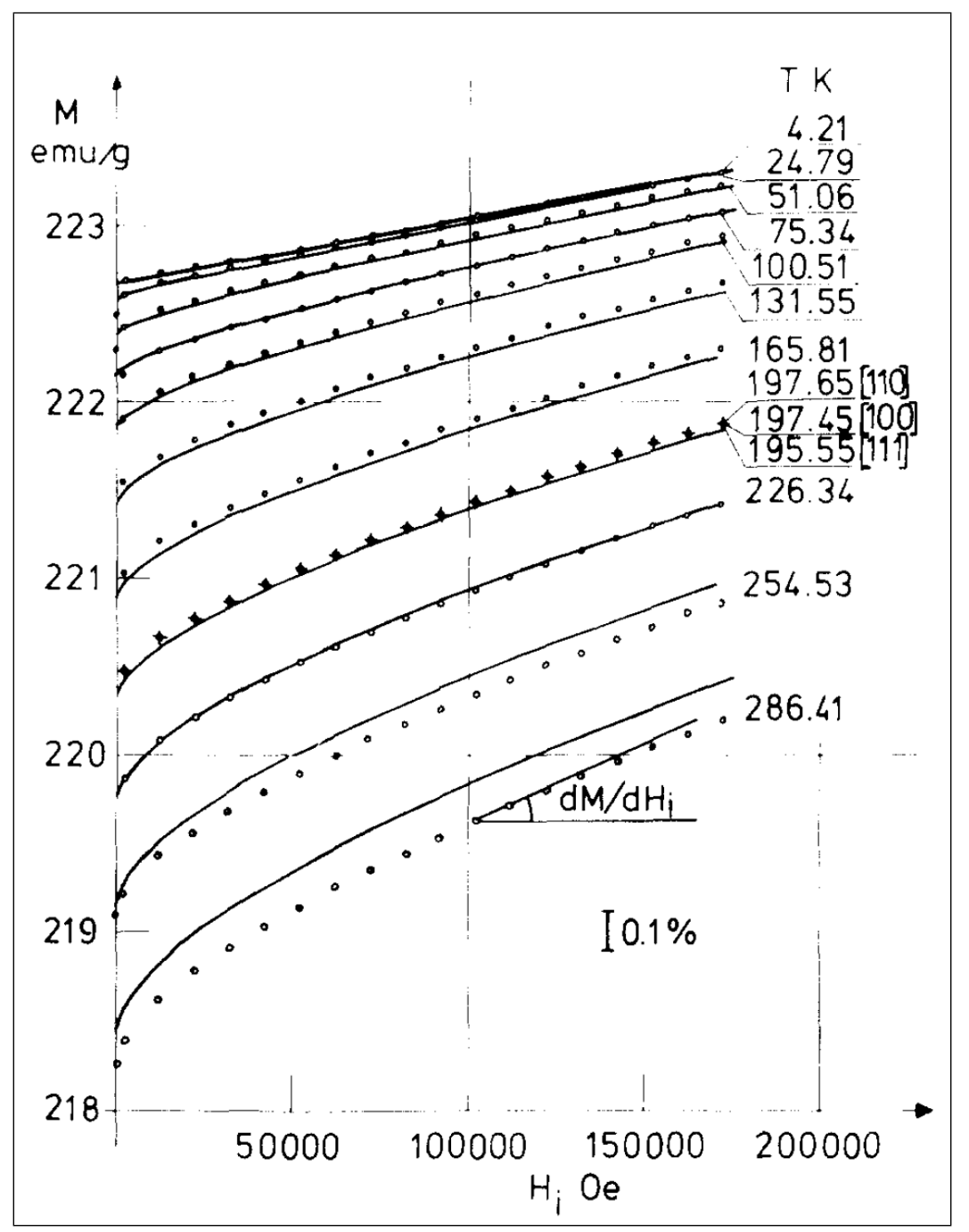

Figure 4.9: High field dependence of magnetization. The $x$-axis is the internal field which is related to the applied field according to Equation 4.29 [15]

Once the saturation magnetization is calculated for high fields and temperature, the spin and orbital contributions to the magnetization need to be separated. A good reference to the procedure was done by Reck and Fry of General Motors in 1969[64]. They used the magnetomechanical ratio $g^{\prime}$ from G.G. Scott (1962)[68] and a total magnetization value of $2.175 \pm 0.01$ from Weiss and Forrer, and Peschard. The approximate expression for the 
total magnetization $M_{t}$, spin magnetization $M_{s}$, and orbital magnetization $M_{o}$ is

$$
\begin{gathered}
\frac{M_{o}}{M_{t}}=\frac{\left(2-g^{\prime}\right)}{g^{\prime}} \\
\frac{M_{s}}{M_{t}}=\frac{2\left(g^{\prime}-1\right)}{g^{\prime}}
\end{gathered}
$$

\subsubsection{Compilation of World Data on Magnetization and g'}

In a new study (2017) at Temple University by Don Jones [16], the world data on iron and nickel was collected in an attempt to obtain the most precise value for the magnetization of the Møller target foils. The study also addressed some concerns with previous works. One concern was the approximation of the electron spin factor, $g_{s p}=2.00231930436182(52)$ being exactly equal to 2 in Equations 4.25 and 4.26. The equations in the more precise form are

$$
\begin{aligned}
& \frac{M_{o}}{M_{t}}=\frac{\left(g_{s p}-g^{\prime}\right)}{g^{\prime}\left(g_{s p}-1\right)} \\
& \frac{M_{s}}{M_{t}}=\frac{g_{s p}\left(g^{\prime}-1\right)}{g^{\prime}\left(g_{s p}-1\right)}
\end{aligned}
$$

which is a $0.11 \%$ decrease in the spin contribution to the magnetization. A similar approximation was made when the magnetization was converted into units of the Bohr magneton, $\mu_{B}$. Here a factor of $g_{s p} / 2$ led to another error of $0.1 \%$.

Another concern was the high field correction done in [14] which used Pauthenet's correction but with the applied field, $H$ instead of the internal field, $H_{i}$. The two are related by

$$
H=H_{i}+\frac{4 \pi M}{\rho}
$$

where $\rho$ is the demagnetization factor which is determined by the shape of the material. For a foil oriented normal to the magnetic field direction, the demagnetization factor is approximately 1 and the demagnetizing field $4 \pi M$ (in cgs units) is equal to the saturation magnetization or about $2.2 \mathrm{~T}$ for Fe. This results in a relation between $H$ and $H_{i}$ for an Fe foil normal to the magnetic field and above saturation as $H_{i}=H-2.2 \mathrm{~T}$. At $H=4 \mathrm{~T}$, well above the saturation for $\mathrm{Fe}, H_{i} \approx 1.8 \mathrm{~T}$. The high field correction in Table 4.2 over estimates the correction by using the applied field. 
The compilation of the world data on Fe is shown in Figure 4.10 along with theoretical fit from [15] in Figure 4.11. The compiled data on nickel, another possible target material is shown in Figure 4.12. The data compiled was for magnetization measurements done at near room temperature conditions thereby eliminating the need to extrapolate data to $0 \mathrm{~K}$ then back up to room temperature. The data was also carefully selected taking into consideration the quality of the data, the different demagnetization factors of the samples used, and the purity of the sample.

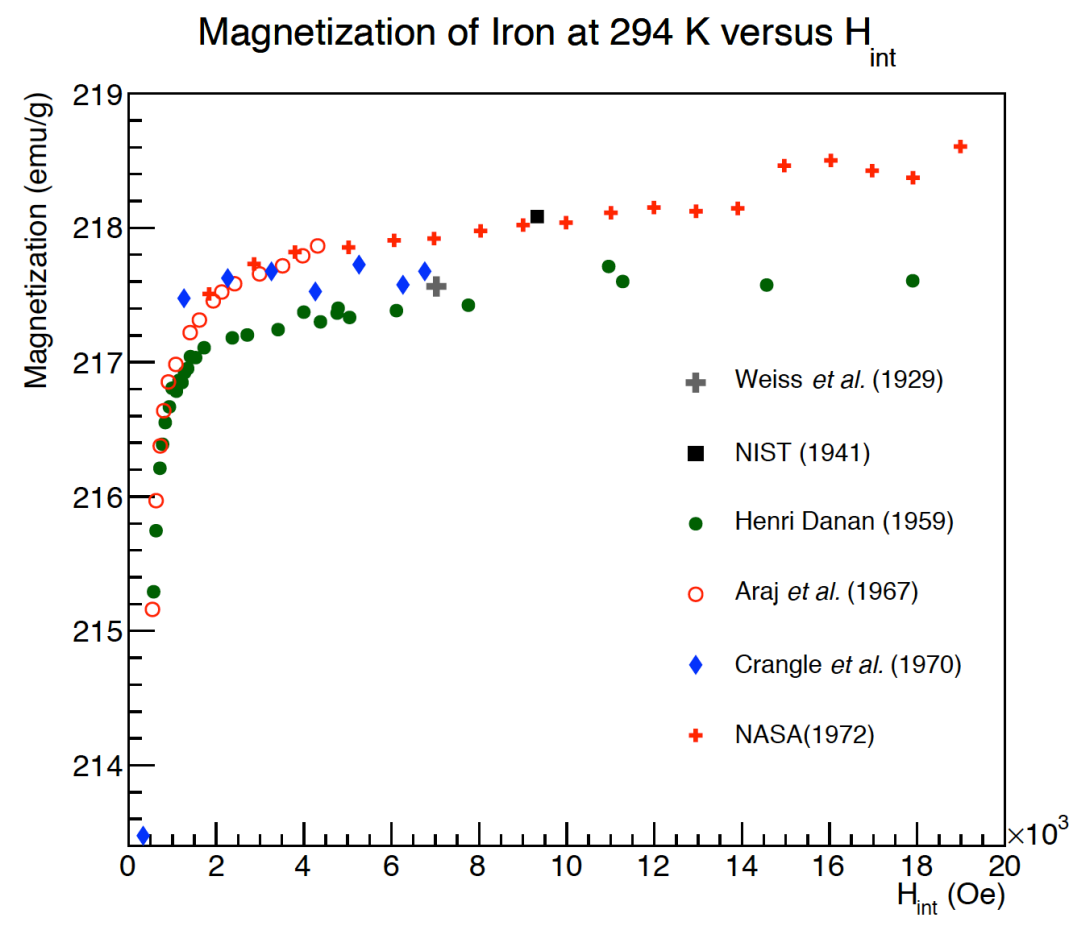

Figure 4.10: Work data on Fe magnetization [16]

The study also compiled the available data on $g^{\prime}$ and the the spectroscopic g-factor, $g$. The two are related by Kittle-Van Vleck equation,

$$
\frac{1}{g^{\prime}}+\frac{1}{g}=1
$$

and provides a nice cross check. An error-weighted fit of the two quantities provide a precise agreement when using Equation 4.30. The compiled data is shown in Figure 4.14 and 4.13.

The results of the Don Jones' study are summarized in Figure 4.15. 


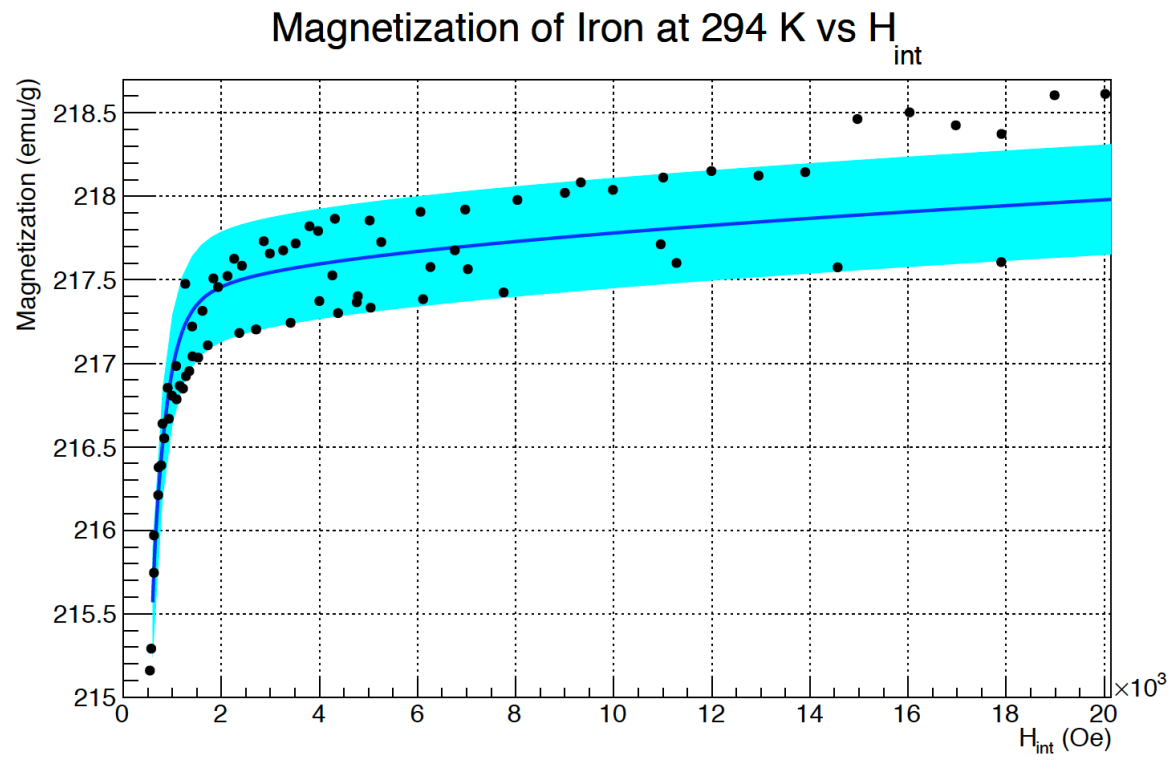

Figure 4.11: Fit of world data for Fe [16]

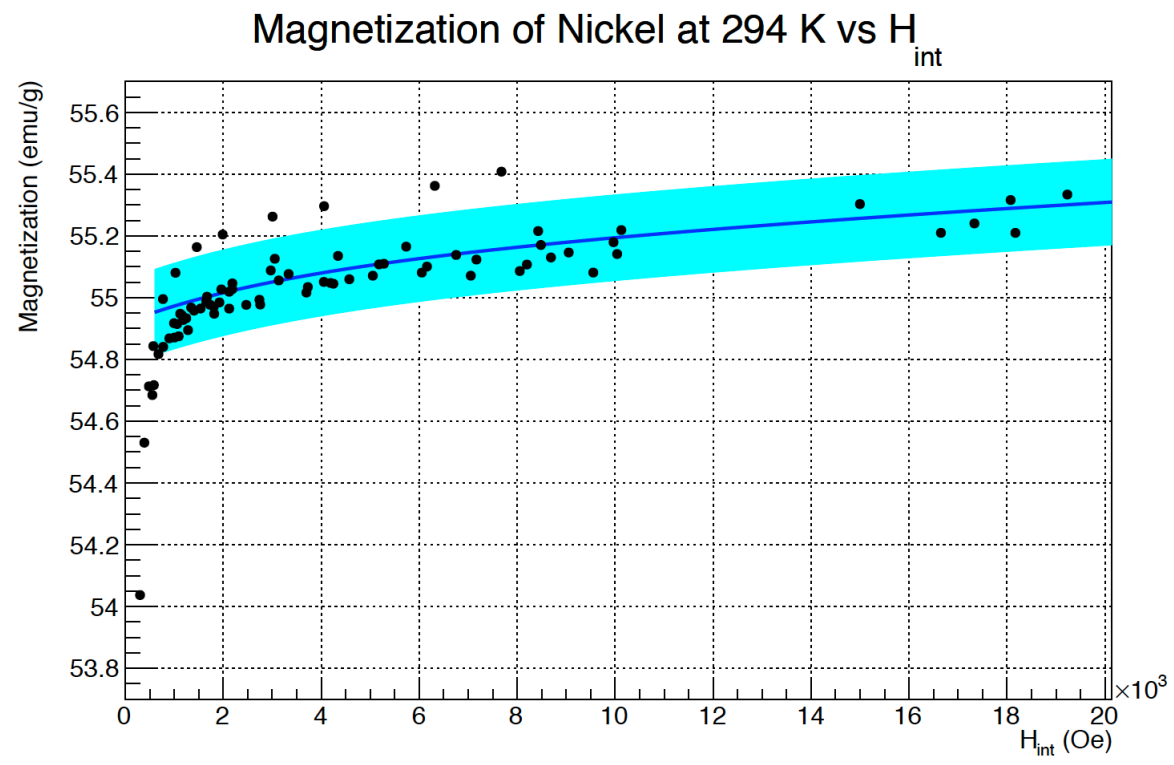

Figure 4.12: Fit of world data for Ni [16] 
Compiled Measurements of Spectroscopic g-factor for Fe

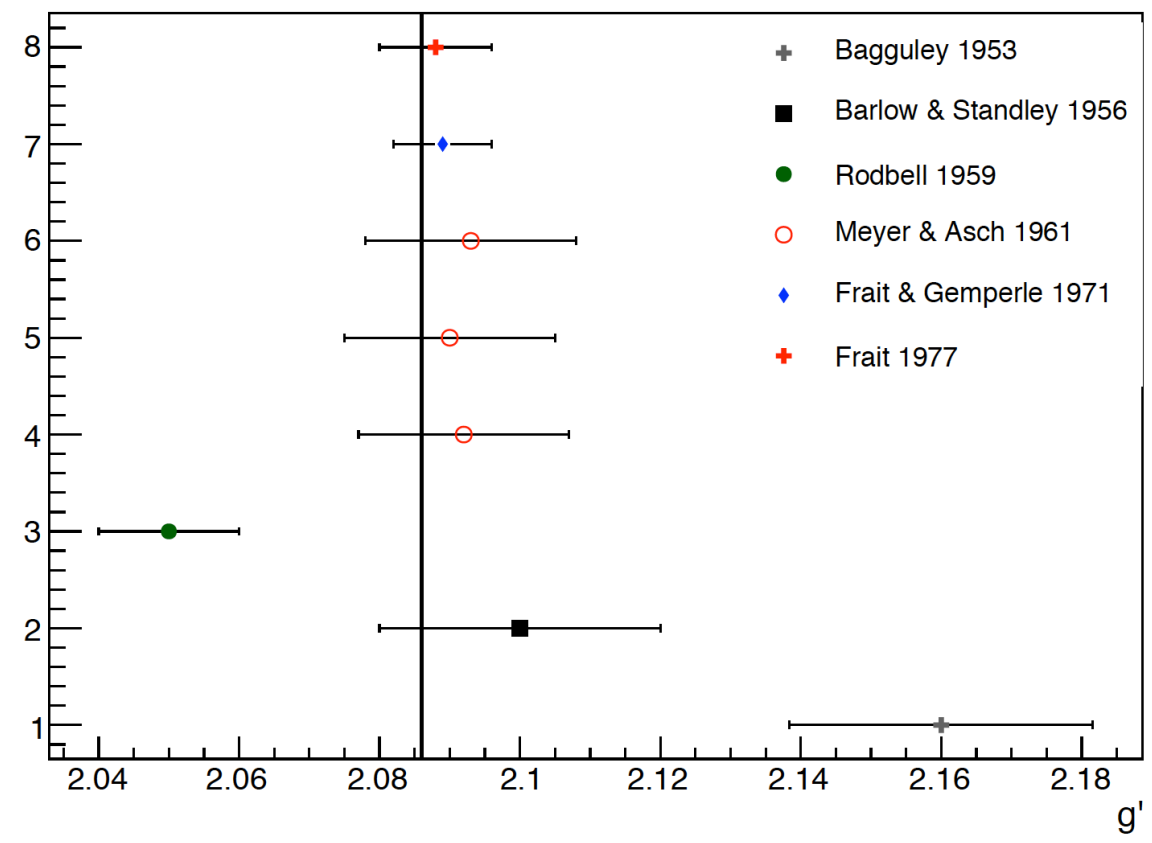

Figure 4.13: Fit of world data for $g$ of Fe [16]

\section{Compiled Measurements of $\mathrm{g}^{\prime}$ for Fe}

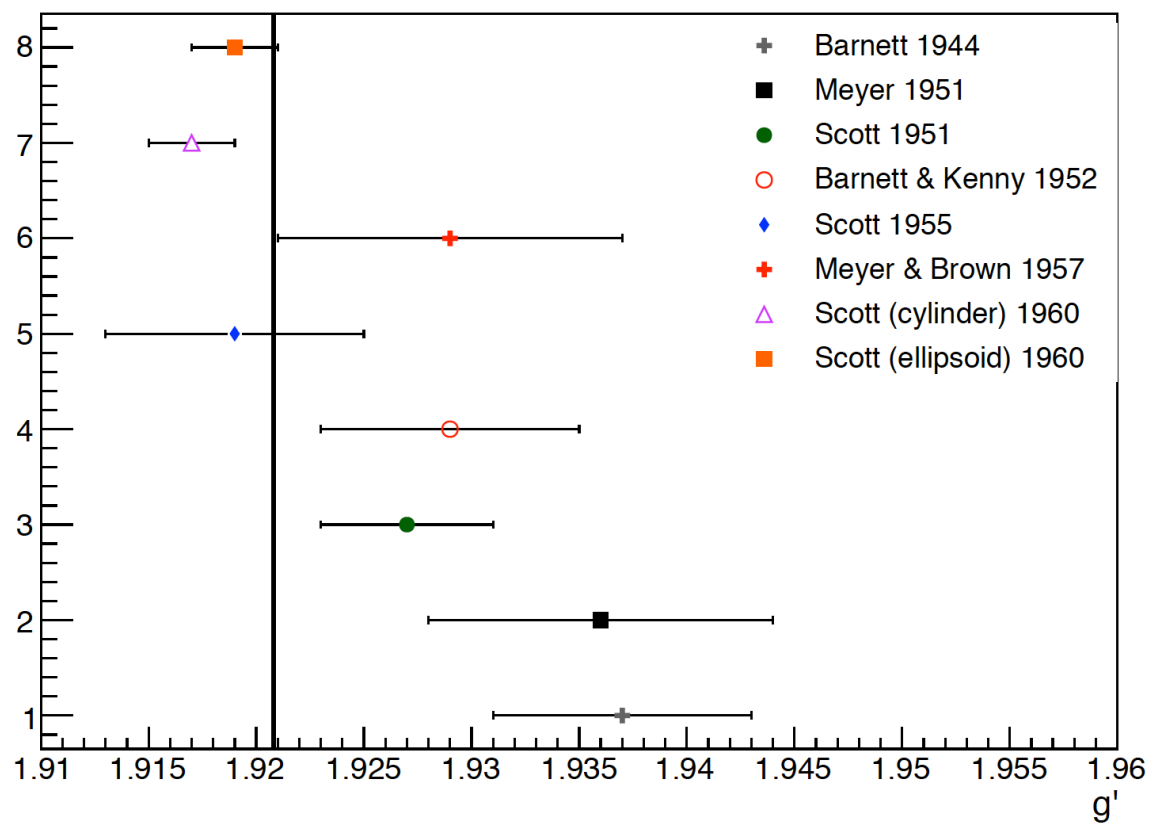

Figure 4.14: Fit of world data for $g^{\prime}$ of Fe [16] 


\begin{tabular}{|l|l|l|c|}
\hline Quantity & Value & Error & Unit \\
\hline Saturation Magnetization $M_{s}$ & 217.95 & 0.33 & $\mathrm{emu} / \mathrm{g}$ \\
Saturation Magnetization $M_{s}$ & 2.1793 & 0.0033 & $\mu_{B} /$ atom \\
$g^{\prime}$ & 1.920 & 0.004 & - \\
$\frac{M_{\text {orb }}}{M_{\text {to }}}=\frac{g_{s p}-g^{\prime}}{g^{\prime}\left(g_{s p}-1\right)}$ & 0.0428 & 0.0022 & - \\
Magnetization from orbital motion $M_{\text {orb }}$ & 0.0932 & 0.0048 & $\mu_{B}$ \\
Magnetization from spin $\left(M_{s}-M_{\text {orb }}\right)$ & 2.0861 & 0.0058 & $\mu_{B}$ \\
Average electron magnetization $(\mathrm{T}=294 \mathrm{~K}, \mathrm{~B}=4 \mathrm{~T})$ & 0.08023 & 0.00022 & $\mu_{B} /$ atom \\
Average electron magnetization $(\mathrm{T}=313 \mathrm{~K}, \mathrm{~B}=4 \mathrm{~T})$ & 0.08008 & 0.00024 & $\mu_{B} /$ atom \\
Average electron polarization $(\mathrm{T}=294 \mathrm{~K}, \mathrm{~B}=4 \mathrm{~T})$ & 0.08014 & 0.00022 & - \\
Average electron polarization $(\mathrm{T}=313 \mathrm{~K}, \mathrm{~B}=4 \mathrm{~T})$ & 0.07999 & 0.00024 & - \\
\hline
\end{tabular}

Figure 4.15: Recommended vales for Fe Target Polarization values for Møller Polarimetry [16]

\subsubsection{Target Angle Dependence}

In 1948 Stoner and Wohlfarth published A Mechanism of Magnetic Hystersis in Heterogeneous Alloys. Their calculations were for prolate spheroids with different demagnetization factors for the equitorial and radial directions. This is the behavior we expect the target polarization to follow as the angle of the foil changes with respect to the magnetic field direction. Figure 4.16 shows how the hysteresis curves change for different foil angles close to $90^{\circ}$. With an applied field of $3 \mathrm{~T}$, a $3^{\circ}$ misalignment of the target foil would lower the magnetization of the foil by $1 \%$, well above our desired uncertainty . Therefore, having the ability to rotate the target foil allows for both precise positioning of the foils and the ability to study the dependence of the target foil angle on the magnetization of the target.

\subsubsection{Target Heating}

The thermal energy deposited from the electron beam can raise the temperature of the target considerably which in turn lowers the magnetization. In order to determine the rise in temperature a CFD calculation was done and shown in Figure 4.17. The calculation was for a $10 \mu \mathrm{m}$ Fe foil with a 10 inch diameter under a $2 \mu \mathrm{A}$ heat load. It was assumed that the target ladder was an infinite heat sink. Here the foil temperature increased $35 \mathrm{~K}$. The calculation also showed that the temperature increase is approximately independent of target thickness and linear with beam current. Using the temperature corrections described in [67], a correction is determined and shown in Figure 4.18 for Fe and Ni foils. 


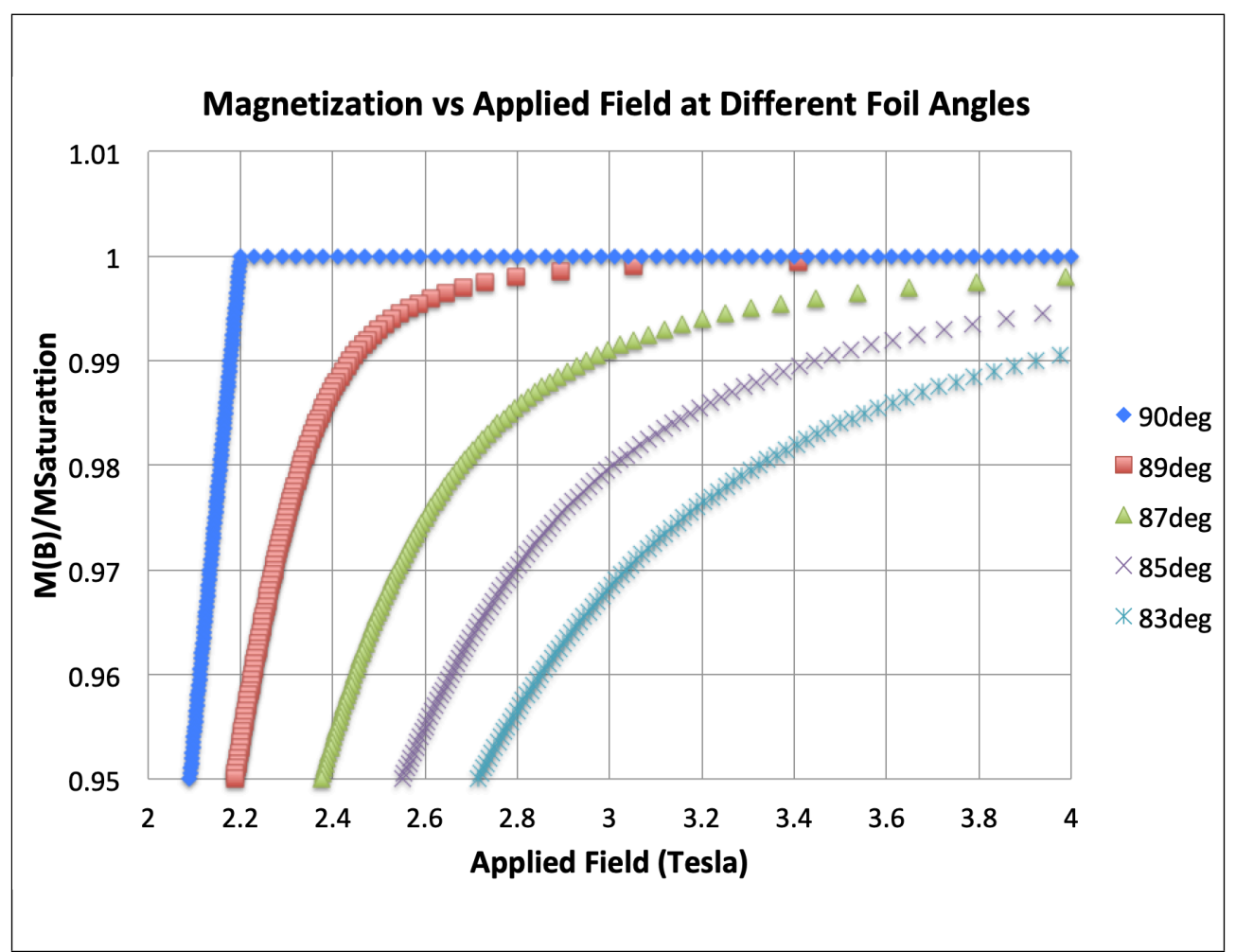

Figure 4.16: Relative foil saturation vs applied field for various angle relative to the applied field.

\subsubsection{XMCD analysis}

X-ray Magnetic Circular Dichroism (XMCD), together with the associated sum rules, allows for an element specific determination of the spin and orbital magnetic moments of various compounds[69]. By taking the difference between two X-ray absorption spectra from a magnetized sample when using left or right circularly polarized photons an XMCD spectrum is constructed. In a collaboration with Alexander Gray's group at Temple University, we performed an XMCD analysis on an amorphous pure iron foil and a permendur foil ( $49 \% \mathrm{Fe}, 49 \% \mathrm{Co}, 2 \% \mathrm{~V}$ ), each foil was $20 \mu \mathrm{m}$ thick. Both of these foils are potential targets for a Moller Polarimeter. The study was performed in order to investigate the possibility of performing precision magnetization measurements with X-rays. The XMCD data was taken at the Advanced Light Source, Lawrence Berkley National Lab. The analysis, results and future opportunities are described in this section.

The data consisted of an X-ray Absorption Spectrum(XAS) and an asymmetry spectrum for each element in each sample (Co and $\mathrm{Fe}$ in permendur and $\mathrm{Fe}$ in iron foil). The XAS 


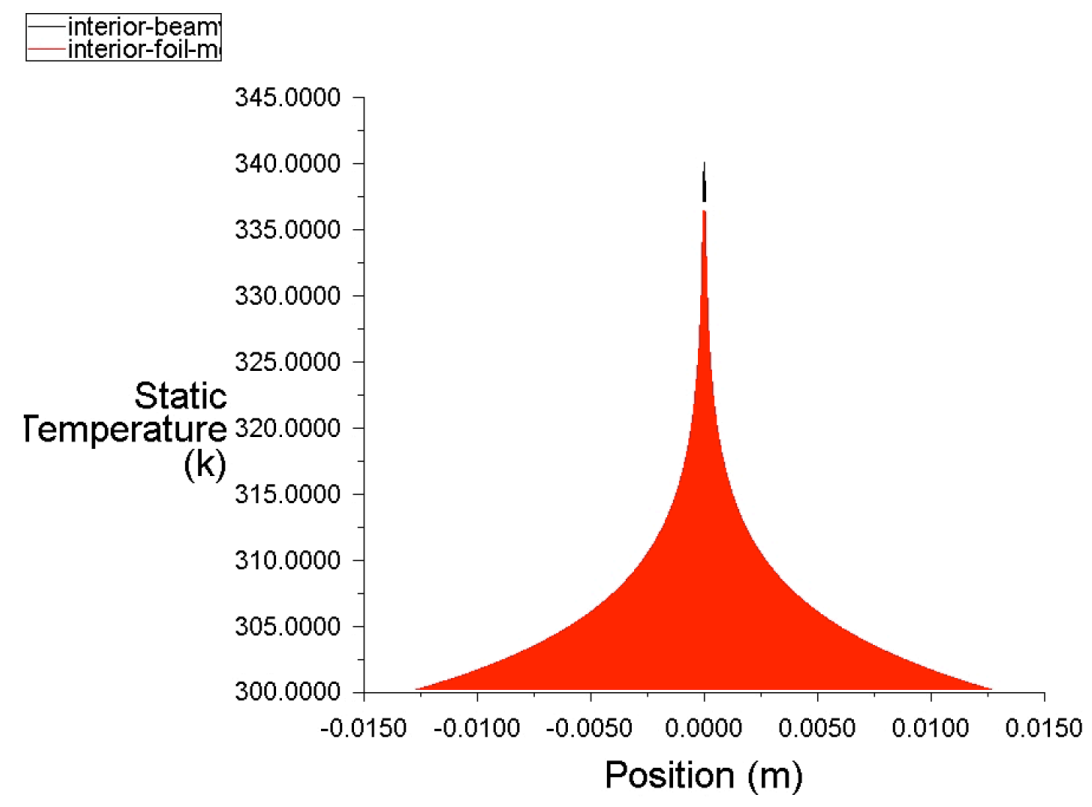

Figure 4.17: CFD calculation of target heating by Silviu Covrig. Assumes $2 \mu \mathrm{A}$ beam, $90 \mu \mathrm{m}$ spot size, on a $10 \mu \mathrm{m}$ Fe foil with a 10 inch diameter which results in a $35 \mathrm{~K}$ increase in temperature.

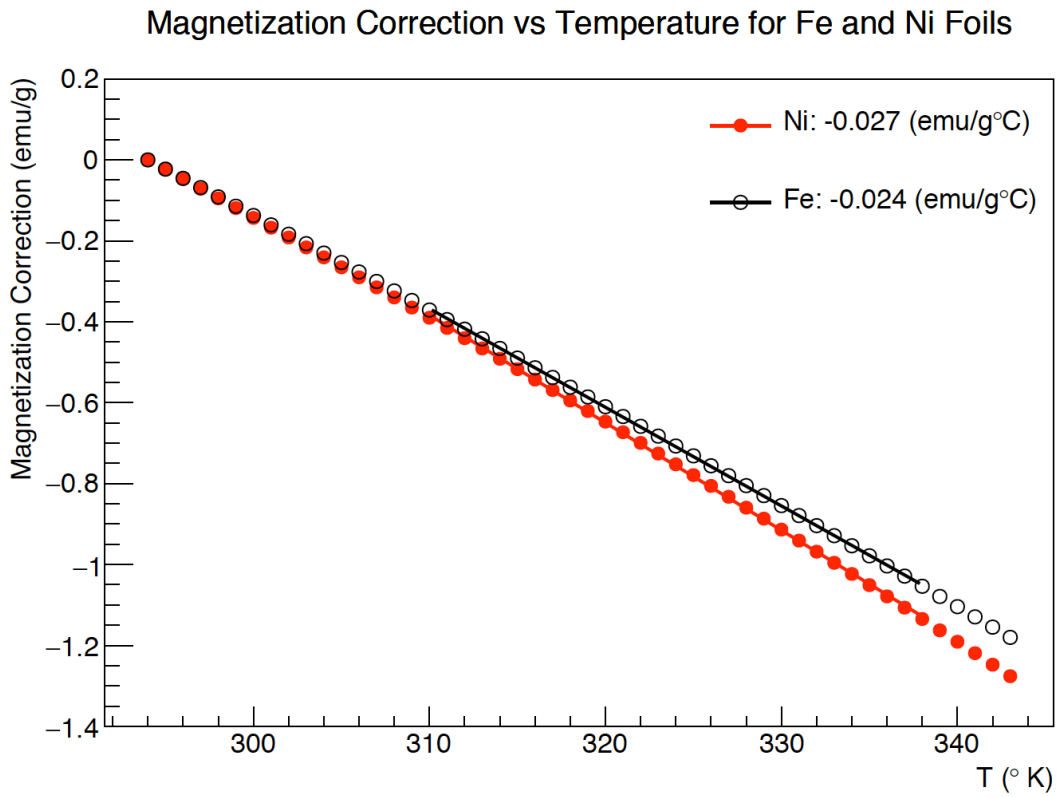

Figure 4.18: Correction to apply to magnetization as a function of beam current 
was the average intensity of both left and right circularly polarized X-rays(lcp and rcp). The asymmetry $(a s y m)$ was defined as $a s y m=\frac{r c p-l c p}{2 * A v g} * 100$. From these two spectra, an XMCD spectrum was created which was the difference between the two circular X-ray polarization absorption spectrums. The sum rules, which provide access to the orbital and spin components of the magnetization, were applied to the XAS and XMCD to obtain the orbital magnetic moment, the spin magnetic moment and the ratio $\frac{m_{\text {orb }}}{m_{s p i n}}$. The sum rules are as follows[70]:

$$
\begin{gathered}
m_{\text {orb }}=-\frac{4 q}{3 r}\left(10-n_{3 d}\right) \\
m_{\text {spin }}=\frac{(6 p-4 q)}{r}\left(10-n_{3 d}\right)\left(1+\frac{7\left\langle T_{z}\right\rangle}{2\left\langle S_{z}\right\rangle}\right)^{-1}
\end{gathered}
$$

with $r$ as the integral of the XAS, $p$ as the integral of the first peak $\left(L_{3}\right)$ in the XMCD, and $q$ as the total integral of both peaks $\left(L_{3}+L_{2}\right)$ in the XMCD. $n_{3 d}$ refers to the number of $3 \mathrm{~d}$ electrons $\left(10-n_{3 d}\right.$ is the number of $3 \mathrm{~d}$ holes). The values used are 6.61 for Fe and 7.51 for Co which are from theoretical calculations[70]. In this analysis we will neglect the $\frac{7\left\langle T_{z}\right\rangle}{2\left\langle S_{z}\right\rangle}$ term for the present time which is small, $-0.4 \%$ for bcc iron and $-.26 \%$ for hcp Co[70] according to first principle band calculations. The term has also been shown to average to 0 in "powder" samples[71]. Dividing the above two equations allows the calculation of the ratio $\frac{m_{\text {orb }}}{m_{s p i n}}$ which does not depend on $n_{3 d}$ and requires only the XMCD spectrum to be integrated.

$$
\frac{m_{\text {orb }}}{m_{\text {spin }}}=\frac{2 q}{9 p-6 q}
$$

The integrated spectra and the application of the sum rules are shown in Figures 4.19.

In order to integrate the XAS, a step function was created to act as a lower integration value (black lines in Figure 4.19). The height of the upper and lower flat portions were chosen to coincide with the spectra beginning and ending values. The middle flat portion was chosen to be $2 / 3$ of the difference between the upper and lower flats in agreement with the quantum degeneracy of the states. The flats were connected by a quarter sine wave. Further analysis is required to determine the dependence of the step function parameters on the sum rule results. The entire XMCD data was shifted vertically before applying the sum rules. This was done in order to guarantee that the integral would settle to a constant value. This is depicted in Figure 4.20. The location of where to take the $\mathrm{p}$ and $q$ values was somewhat arbitrary. The q value (the integral of both peaks) at first appeared to level off after the shift was applied however even small changes in q significantly effect the results. 

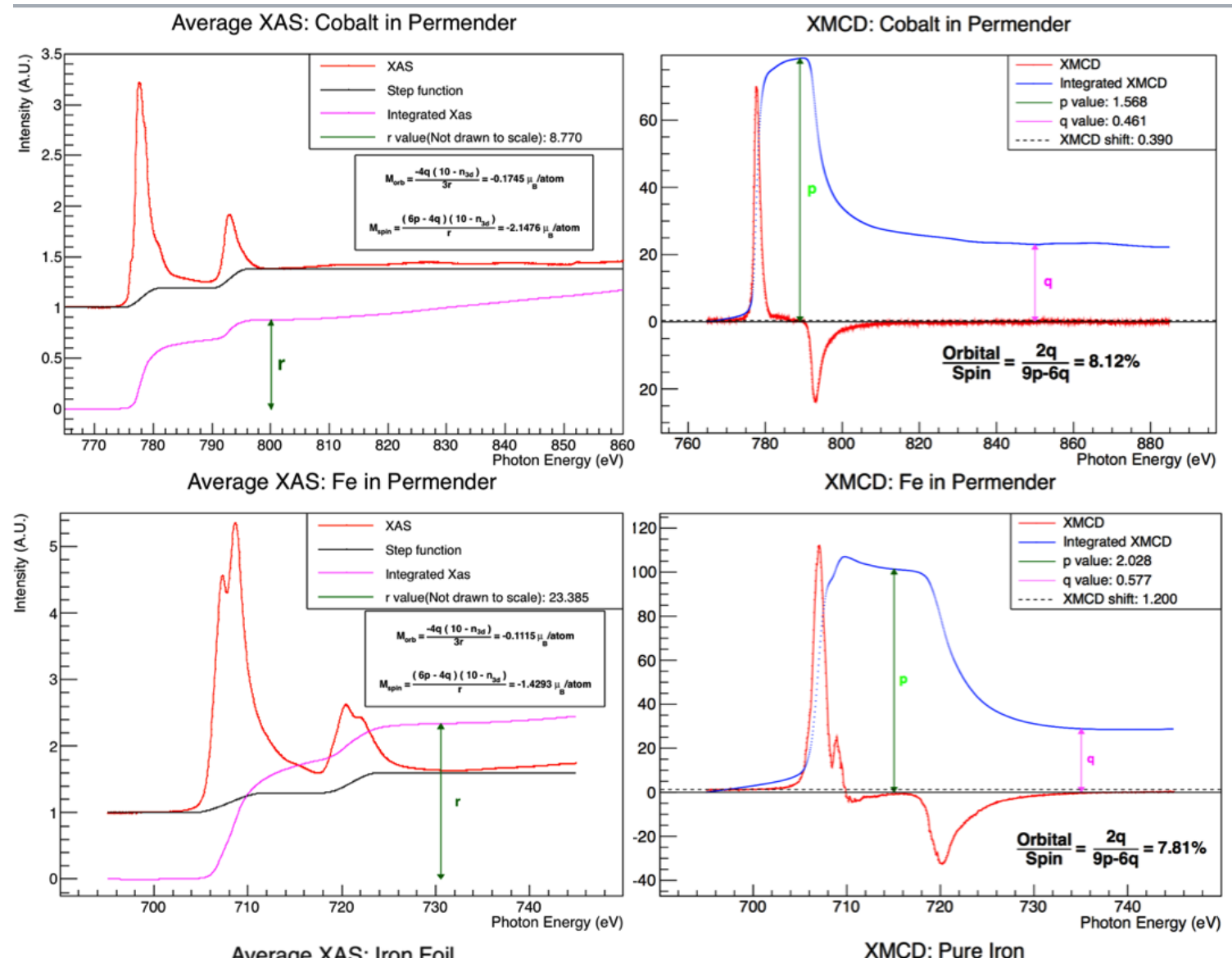

Average XAS: Iron Foil
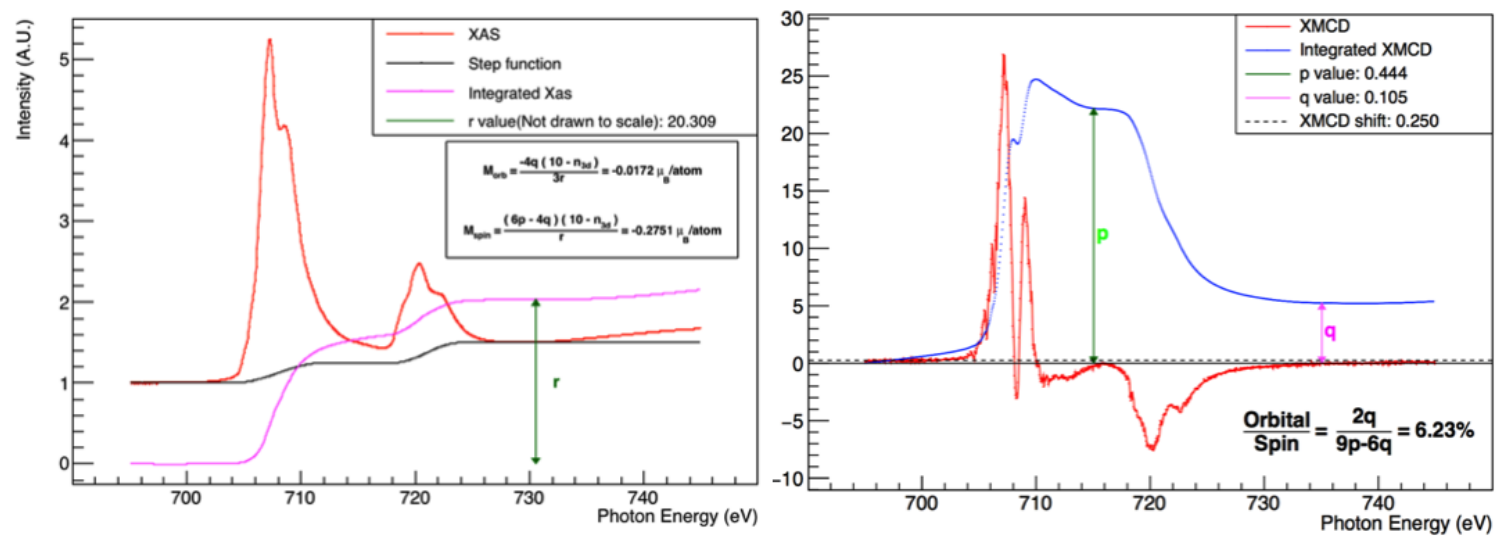

Figure 4.19: Analysis of $\mathrm{XAS}$ (left) and $\mathrm{XMCD}$ (right) spectrum using the sum rules 
XMCD: Pure Iron

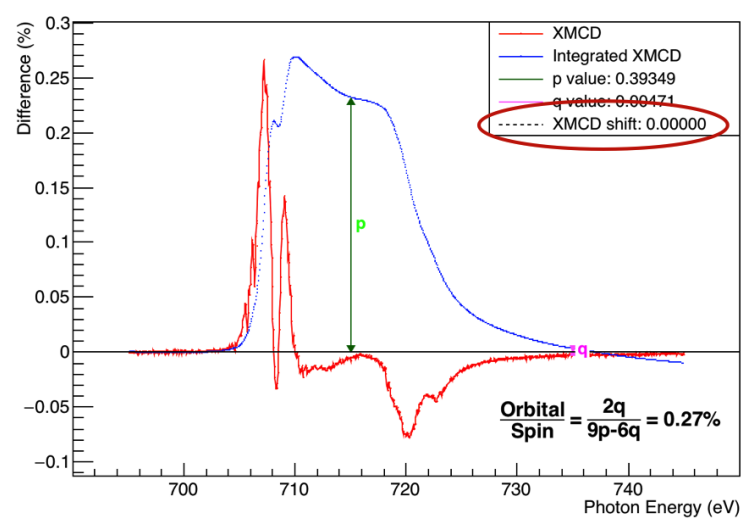

XMCD: Pure Iron

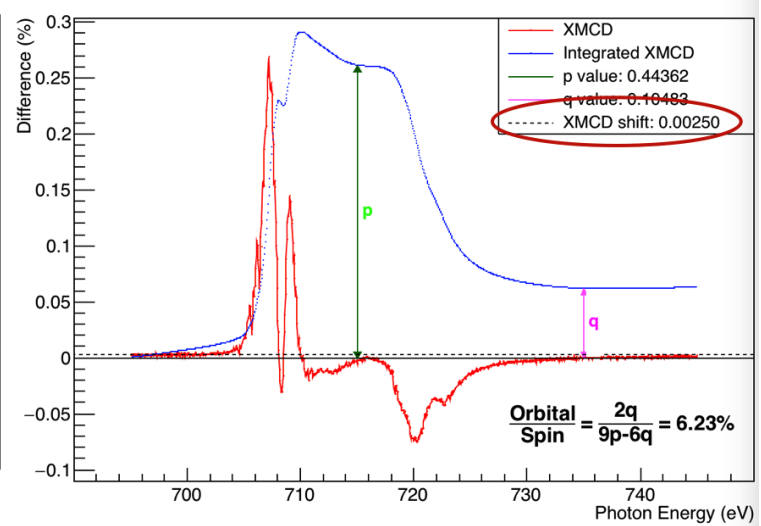

Figure 4.20: Left: Integration(blue curve) without shift Right:Integration with spectrum shifted vertically

The $\mathrm{p}$ value is to be taken after the $L_{3}$ peak which is also not well defined. Figure 4.21 shows how the ratio changes with the $\mathrm{p}$ and $\mathrm{q}$ locations.

The iron spectra lacked a single pronounced $L_{2}$ and $L_{3}$ peak. This was due to the presence of iron oxides on the surface. Since the electron yield XMCD technique probes only the first several nanometers of the foil the oxide on the surface contributed to a large part of the signal. Several approaches were taken to analyze the oxide. In the first, the spectra were fit to two Voigt functions. Figure 4 shows the fit which did not improve our final results. For the second approach, data was digitized of existing iron oxide spectra.[72] Then the individual oxide spectra were fit to the iron foil spectrum. The fit is shown in Figure 4.23 and indicates that the signal had a relatively large oxide component.

The table below summarizes the results from Figure 4.19 and the magnetization values that are currently being used to determine the polarization at JLab. These reference values are also frequently cited in other papers by the solid state community. It should be noted that the magnetization values are for saturated samples, which is not the case for our samples at 0.5T. Figure 4.24 shows our results compared with a recent (2016) paper[73] in which the sample was a $110 \mathrm{Fe}$ film $(20 \mu \mathrm{m})$ at various field strengths.

It is still to be determined if the sum rules can provide the precision required to verify or better the current values of spin and orbital magnetic moment in iron and other $3 \mathrm{~d}$ transition metals. Since the ratio, $m_{\text {orb }} / m_{\text {spin }}$ does not depend on $n_{3 d}$ this seems the most promising. Knowing this ratio, gives us $g^{\prime}$ which can be compared to earlier works. Several experimental factors can be made to improve results. 

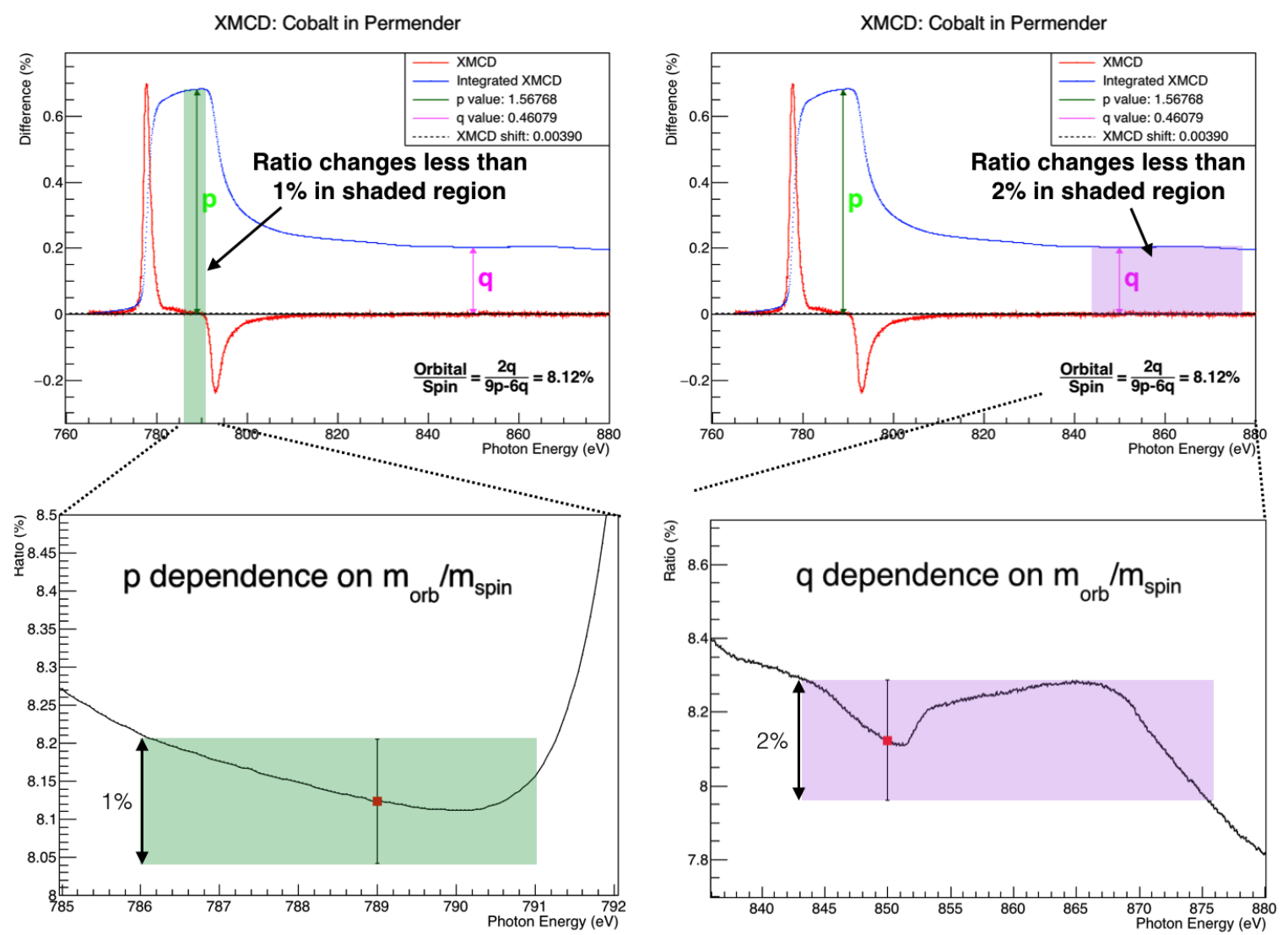

Figure 4.21: $\frac{m_{o}}{m_{s}}$ dependence on the location of $\mathrm{p}$ and $\mathrm{q}$

\begin{tabular}{||ccccc||}
\hline Material & $M_{o}\left(\frac{\mu_{B}}{\text { atom }}\right)$ & $M_{s}\left(\frac{\mu_{B}}{\text { atom }}\right)$ & $M_{T}\left(\frac{\mu_{B}}{\text { atom }}\right)$ & $\frac{M_{o}}{M_{s}}$ \\
\hline \hline Co in Permendur & 0.1745 & 2.1476 & 2.3221 & $8.12 \%$ \\
\hline Co reference(Saturated) & 0.147 & 1.52 & 1.66 & $9.7 \%$ \\
\hline \hline Fe in Permendur & 0.1115 & 1.4293 & 1.5408 & $7.81 \%$ \\
\hline Fe Foil & 0.0234 & 0.3746 & 0.3980 & $6.23 \%$ \\
\hline Fe reference(Saturated) & 0.092 & 2.08 & 2.17 & $4.4 \%$ \\
\hline
\end{tabular}

Table 4.3: Summary of results with referenced values coming from [18] 

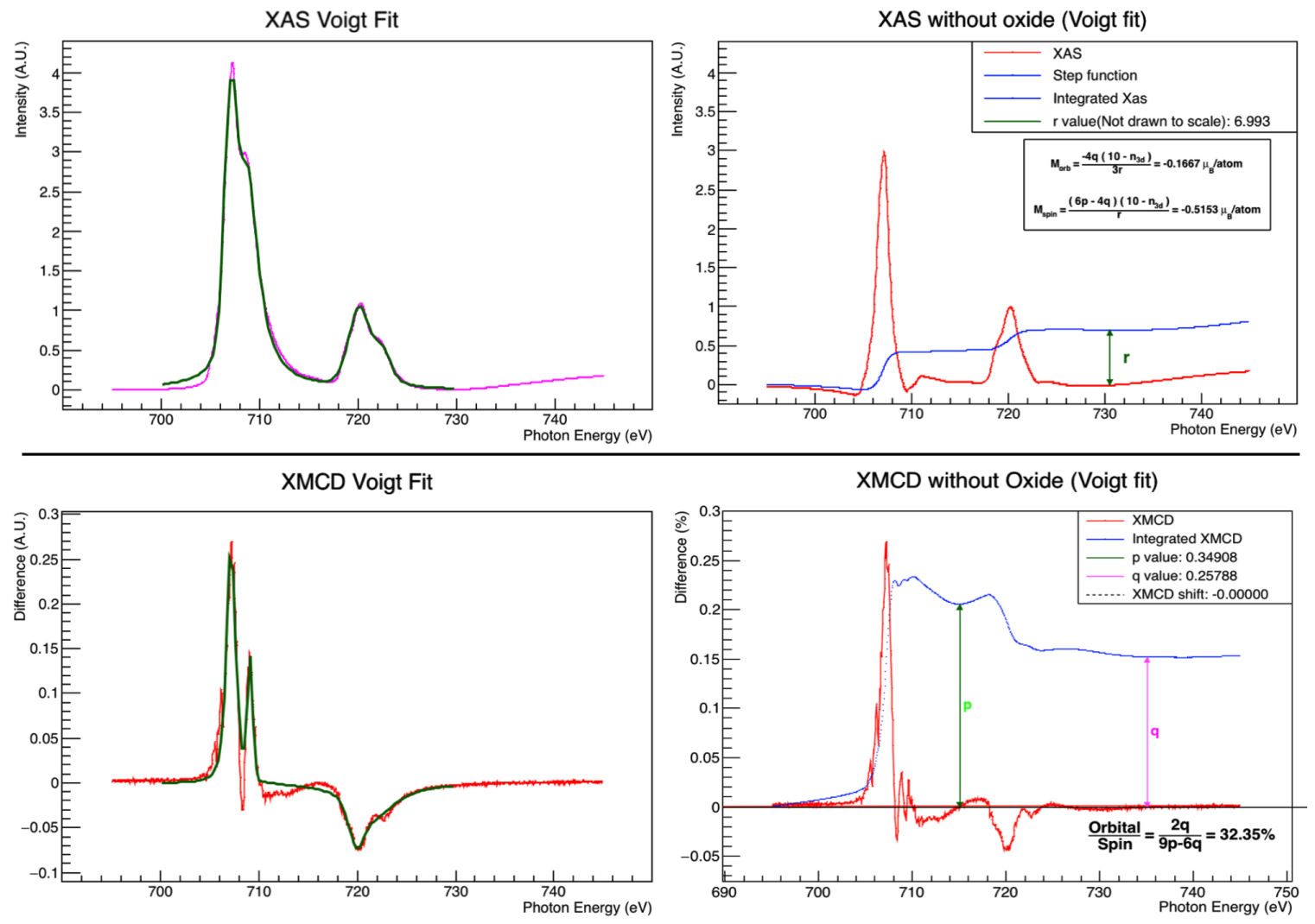

Figure 4.22: Data fit to Voigt functions
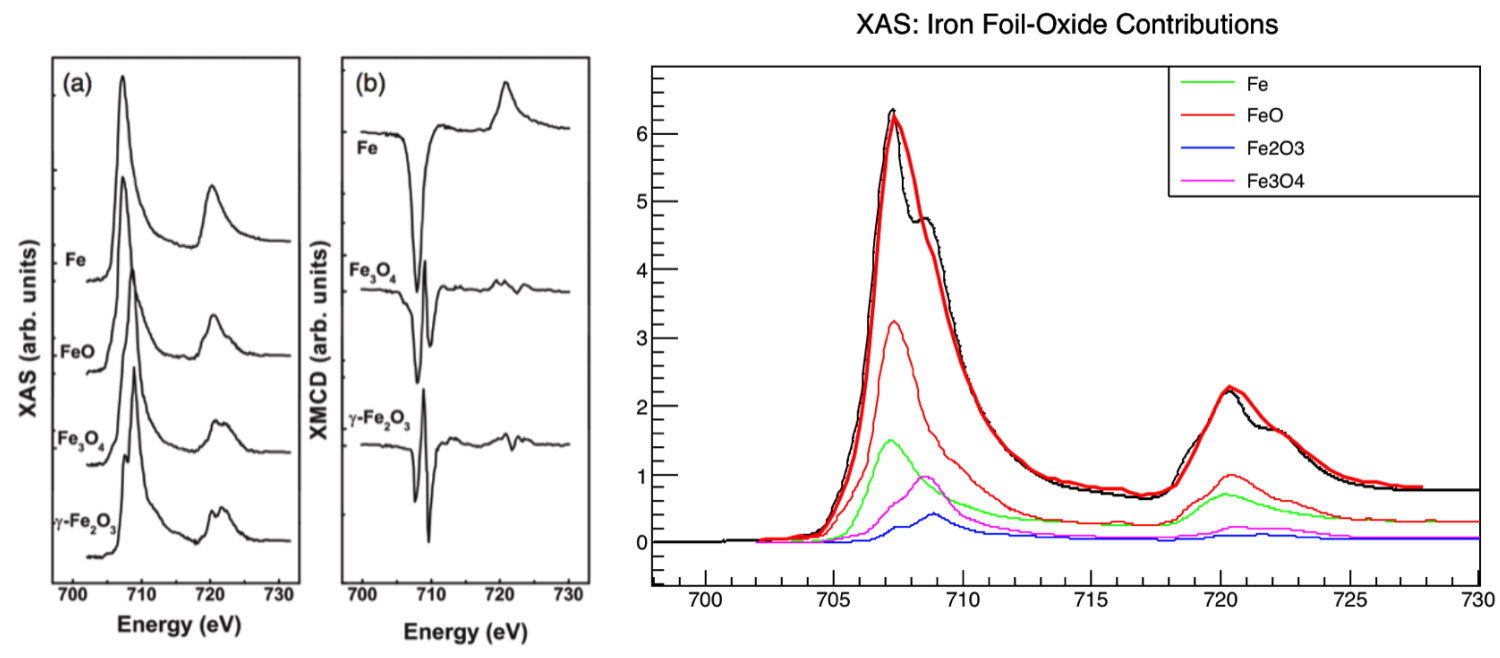

Figure 4.23: Various oxide contributions to iron foil spectrum 

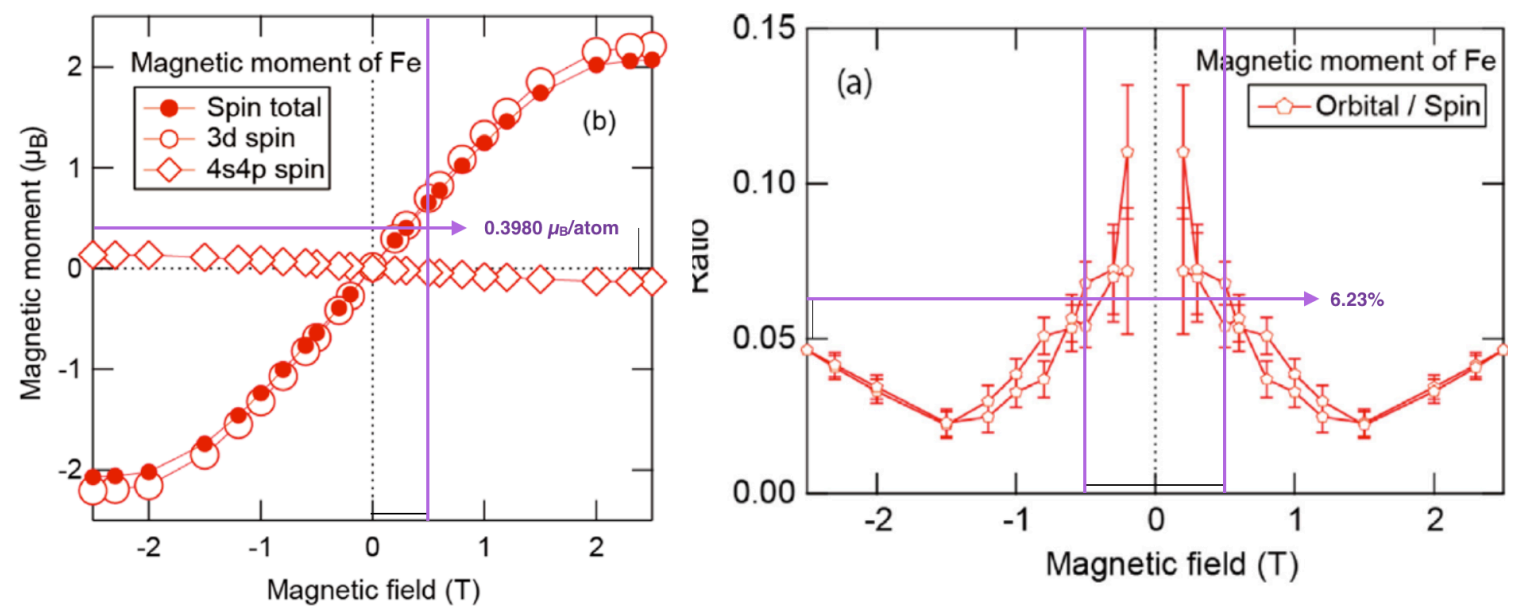

Figure 4.24: The magnetization and orbital to spin magnetic moment ratio from Mössbaurer spectroscopy and a magnetic compton profile.Cite The purple lines show our values calculated from the sum rules at $.5 \mathrm{~T}$.

- Increasing the field to greater than $2.2 \mathrm{~T}$ would allow comparison with saturation data

- Treat the sample with an acid to remove the oxide layer

- XMCD measurements on pure Co and Ni samples

- XMCD measurements in the transmission configuration would allow the full depth of the foil to be probed but this would require a foil thickness on the nanometer scale, the thinnest available from Goodfellows is $1 \mu \mathrm{m}$. Alternately an iron foil could be deposited on a substrate.

\subsection{Analyzing Power}

The size of the asymmetry in a polarization measurement is directly proportional to the mean analyzing power, $\left\langle A_{z z}\right\rangle$. If the detector only accepted $\theta_{C M}=90^{\circ}$ electrons then the analyzing power would be exactly 7/9. In practice however the acceptance is in a range of $\theta_{C M}$, usually centered at $90^{\circ}$. Figure 4.25 shows the acceptance and $\left\langle A_{z z}\right\rangle$ for a particular tune at $2.2 \mathrm{GeV}$, the beam energy for CREX. Here $\left\langle A_{z z}\right\rangle=0.7362, \sim 5 \%$ lower than $7 / 9$, and the $\theta_{C M}$ acceptance is $\pm 20^{\circ}$. There are many factors that effect the analyzing power and they must be accurately simulated. In the following sections I will study the sensitivities of the various factors including beam position, magnetic field strengths and ill-defined geometries. 
Acceptance (Coincidence)

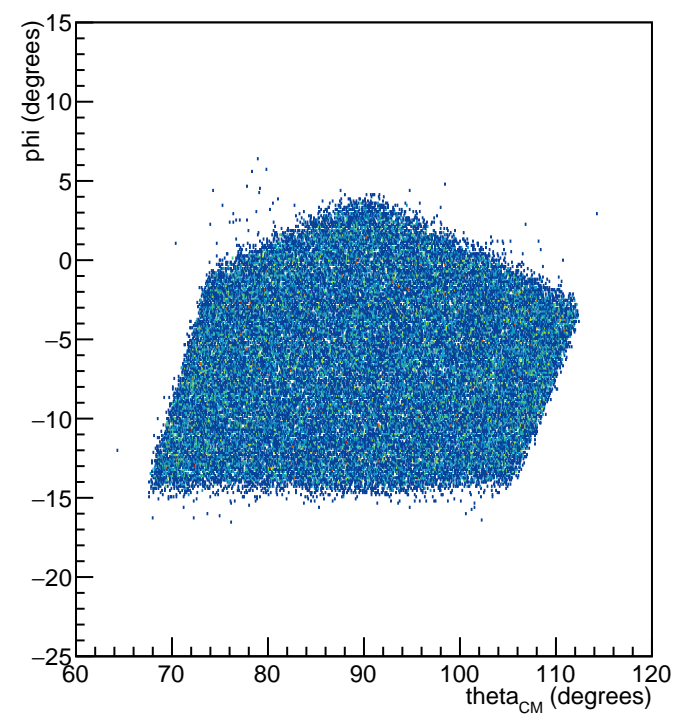

Analyzing Power (Coincidence)

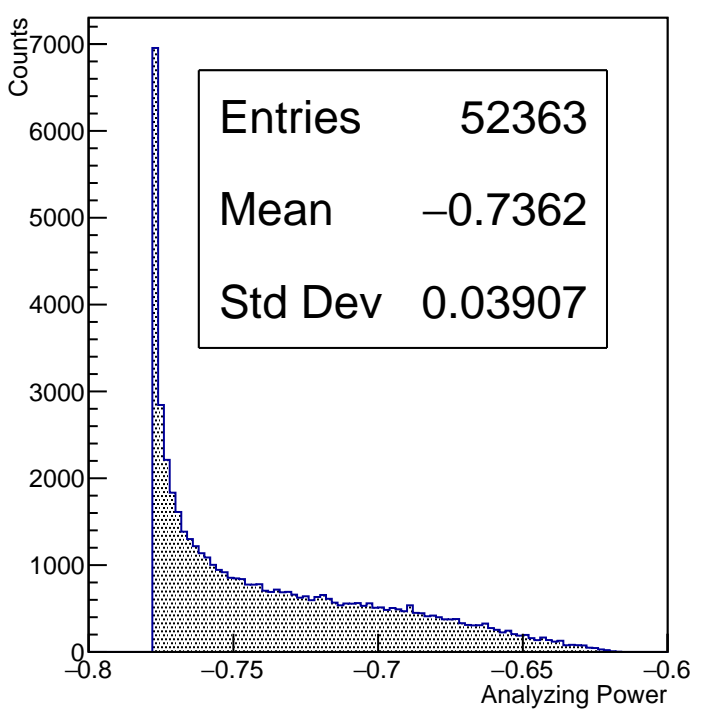

Figure 4.25: Acceptance and analyzing power for $E_{B E A M}=2.22 \mathrm{GeV}$. The color scale on the left represents the number of electrons in each bin with blue being the fewest and the redder regions being the most.

\subsubsection{Beam Position}

The position of the incoming beam is generally stable at the $100 \mu \mathrm{m}$ level. Adjusting the beam position in the simulation and plotting $\left\langle A_{z z}\right\rangle$ as a function of position allows the sensitivity to be quantified. Figures 4.26 and 4.27 show the dependence of the beam position on the rates and $\left\langle A_{z z}\right\rangle$. Assuming a generous uncertainty of $0.2 \mathrm{~mm}$ in the $\mathrm{x}$ and $\mathrm{y}$ position of the beam leads to a uncertainity in $\left\langle A_{z z}\right\rangle$ of $0.05 \%$ and $0.08 \%$ respectively.

\subsubsection{Beam Energy}

Beam energy stability can also affect the acceptance of the detector thus $\left\langle A_{z z}\right\rangle$. Variations in the beam energy/momentum are expected to be at the $10^{-4}$ level. Figure 4.28 shows the sensitivities as a $2.2 \mathrm{GeV}$ beam is varied by \pm 1 and $2 \mathrm{MeV}$. An uncertainty of $\frac{\delta p}{p}=$ $2.5 \times 10^{-4}$ corresponds to an uncertainty in $\left\langle A_{z z}\right\rangle$ of $0.01 \%$. 

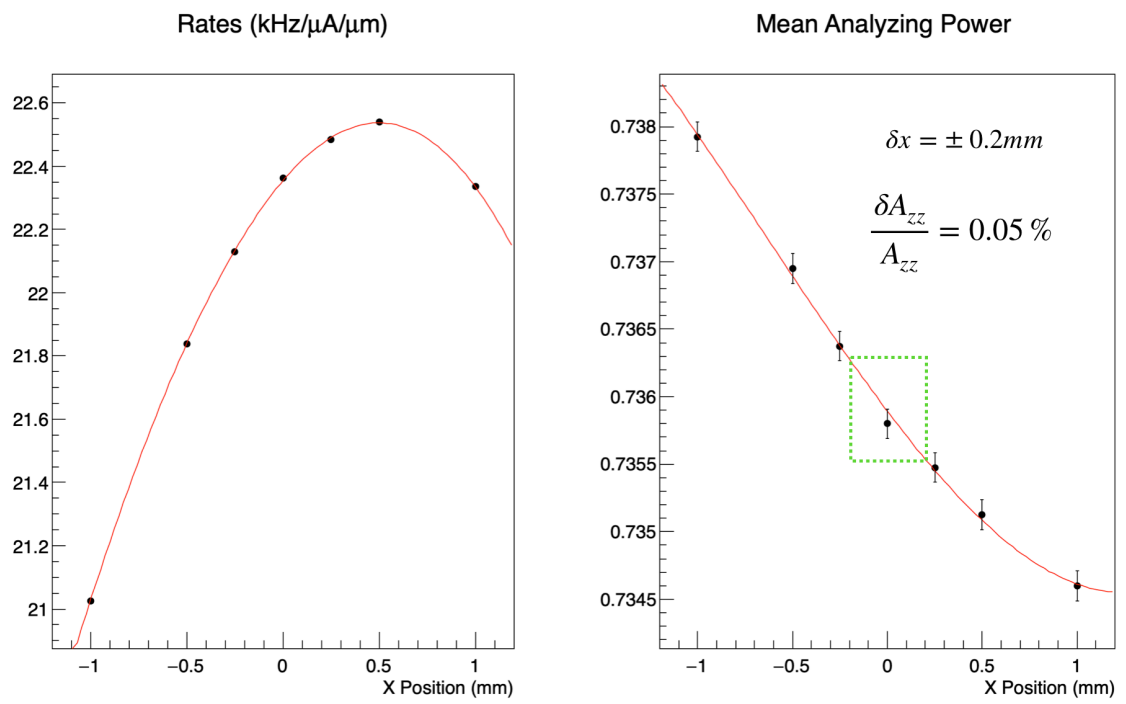

Figure 4.26: $\mathrm{x}$ beam position dependence on $\left\langle A_{z z}\right\rangle$ and rates. The width of the green box represents the uncertainty of the $\mathrm{x}$-axis and the height the corresponding uncertainty of $\left\langle A_{z z}\right\rangle$.
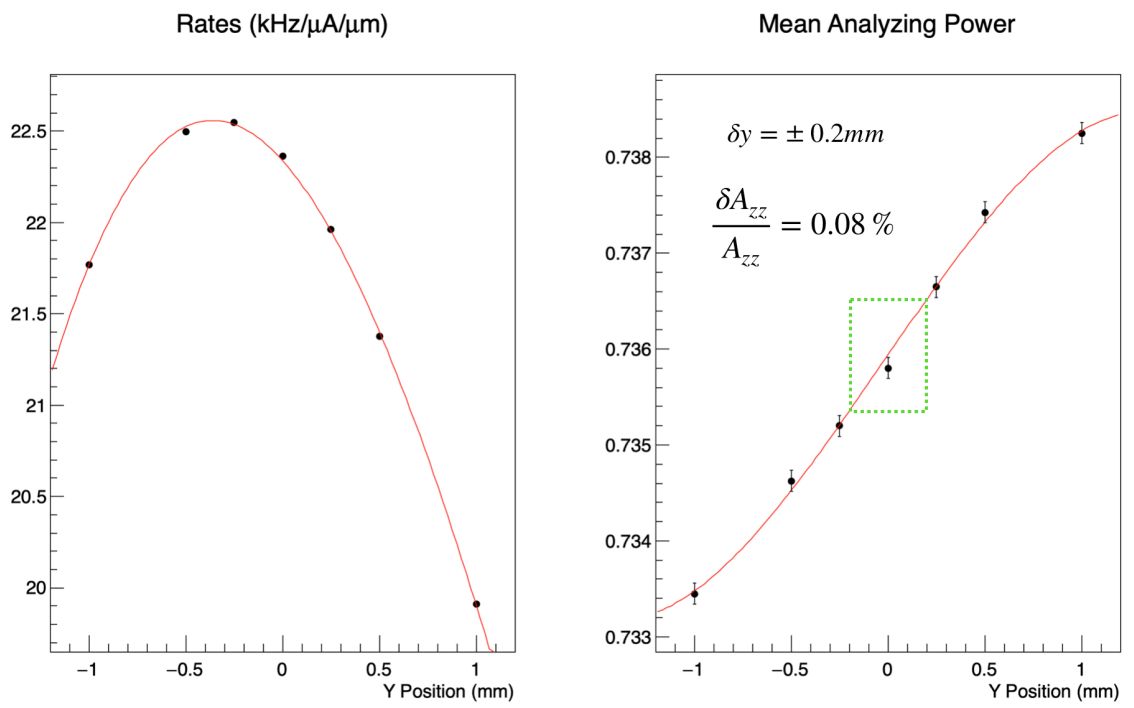

Figure 4.27: y beam position dependence on $\left\langle A_{z z}\right\rangle$ and rates. The width of the green box represents the uncertainty of the $\mathrm{x}$-axis and the height the corresponding uncertainty of $\left\langle A_{z z}\right\rangle$. 

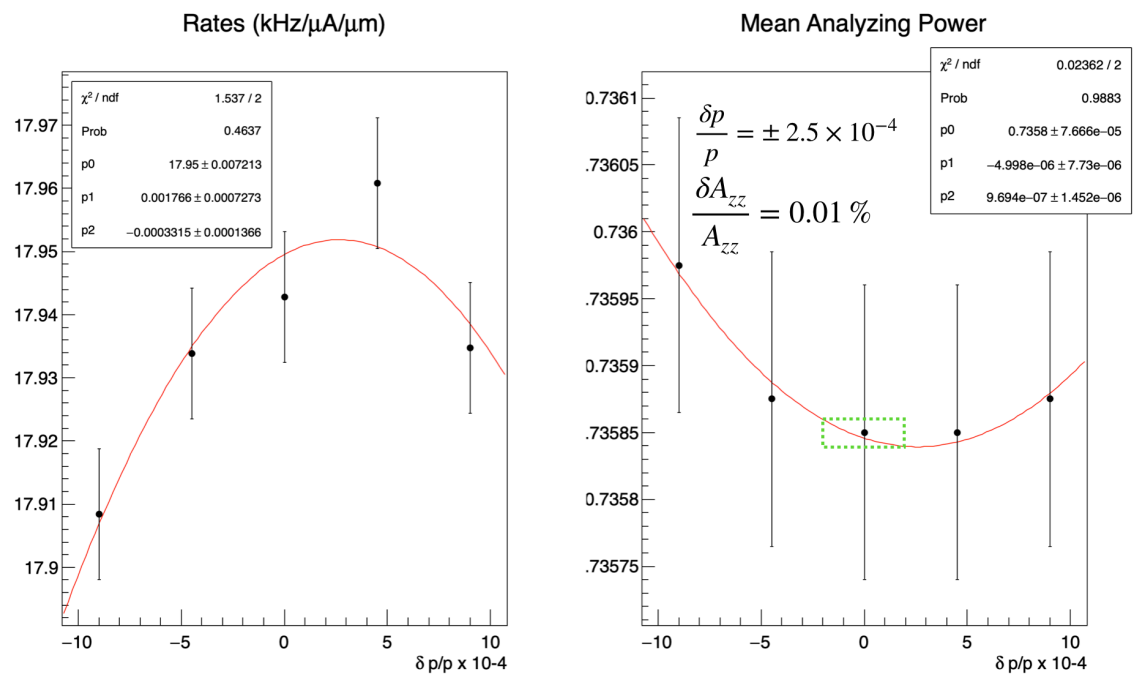

Figure 4.28: Beam energy/momentum dependence on $\left\langle A_{z z}\right\rangle$ and rates. The width of the green box represents the uncertainty of the x-axis and the height the corresponding uncertainty of $\left\langle A_{z z}\right\rangle$. 


\subsubsection{Detector Geometry}

The position of the detector elements contribute to the acceptance of the detector and their precise location should be well known. JLab routinely has beam line components surveyed by their alignment group which provides the location of various elements relative to a fixed coordinate system. The precision of these measurements are at the $100 \mu \mathrm{m}$ level. The position of the detector/calorimeter was measured in 2019, however most of the measurements were made at the back of the assembly and limited measurements were done at the face of the detector. The uncertainty of the final position of the face of the detector is estimated to be $\pm 2 \mathrm{~mm}$. Figures 4.30 and 4.31 shows the effect of moving the detector in $\mathrm{x}$ (horizontally) and y (vertically). An uncertainty in $\mathrm{x}$ of $\pm 2 \mathrm{~mm}$ corresponds to an uncertainty in $\left\langle A_{z z}\right\rangle$ of $0.01 \%$ and an uncertainty in y of $\pm 2 \mathrm{~mm}$ corresponds to an uncertainty in $\left\langle A_{z z}\right\rangle$ of $0.05 \%$. The horizontal position is less sensitive because the detector is approximately $3 \mathrm{~cm}$ wider than the opening in the shielding box that the electrons pass through. However the y position is more sensitive to changes because the bottom edge of the calorimeter is defining part of the acceptance. The shielding box, detector and entrance are illustrated in Figure 4.29.

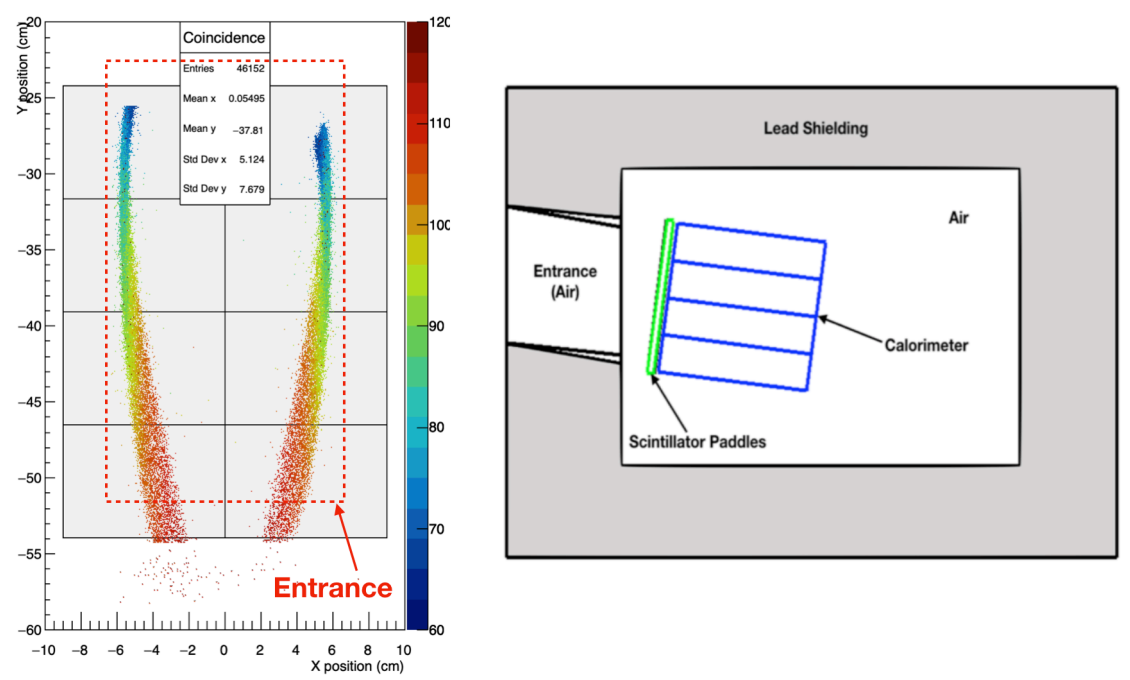

Figure 4.29: (Left) Coincidence electron locations on the calorimeter blocks(grey) color coded by energy $\left(\theta_{C M}\right)$. The blue electrons are the highest energy while the red are the lowest. The red dashed line is the outline of the opening in the lead shielding that surrounds the detector.(Right) Side view of the calorimeter, entrance opening, and lead shielding box. The position of the detector and entrance were separately shifted in y (up and down in figure) and $\mathrm{x}$ (in and out of page) and the change in the analyzing power and rates were found. 
The location of the entrance in the lead shielding box also can effect the acceptance. Figures 4.32 and 4.33 shows the effects of moving the location of the entrance in $\mathrm{x}$ and $\mathrm{y}$. An uncertainty in $\mathrm{x}$ of $\pm 1 \mathrm{~mm}$ corresponds to an uncertainty in $\left\langle A_{z z}\right\rangle$ of $0.02 \%$ and an uncertainty in y of $\pm 1 \mathrm{~mm}$ corresponds to an uncertainty in $\left\langle A_{z z}\right\rangle$ of $0.03 \%$.
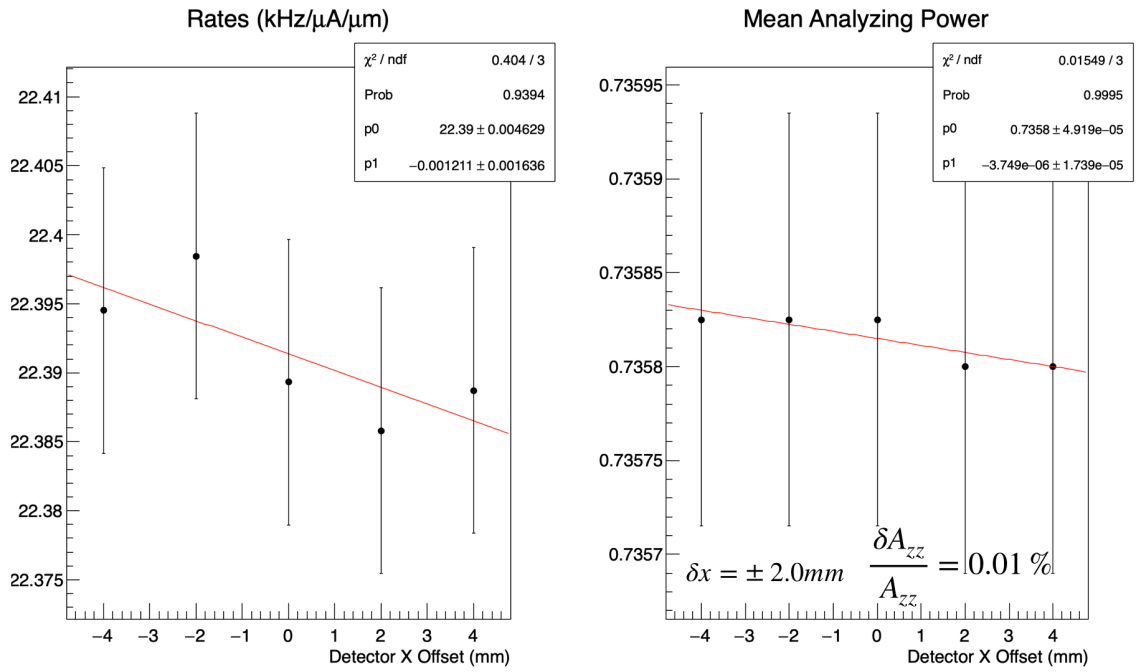

Figure 4.30: $\mathrm{x}$ detector position dependence on $\left\langle A_{z z}\right\rangle$ and rates. 

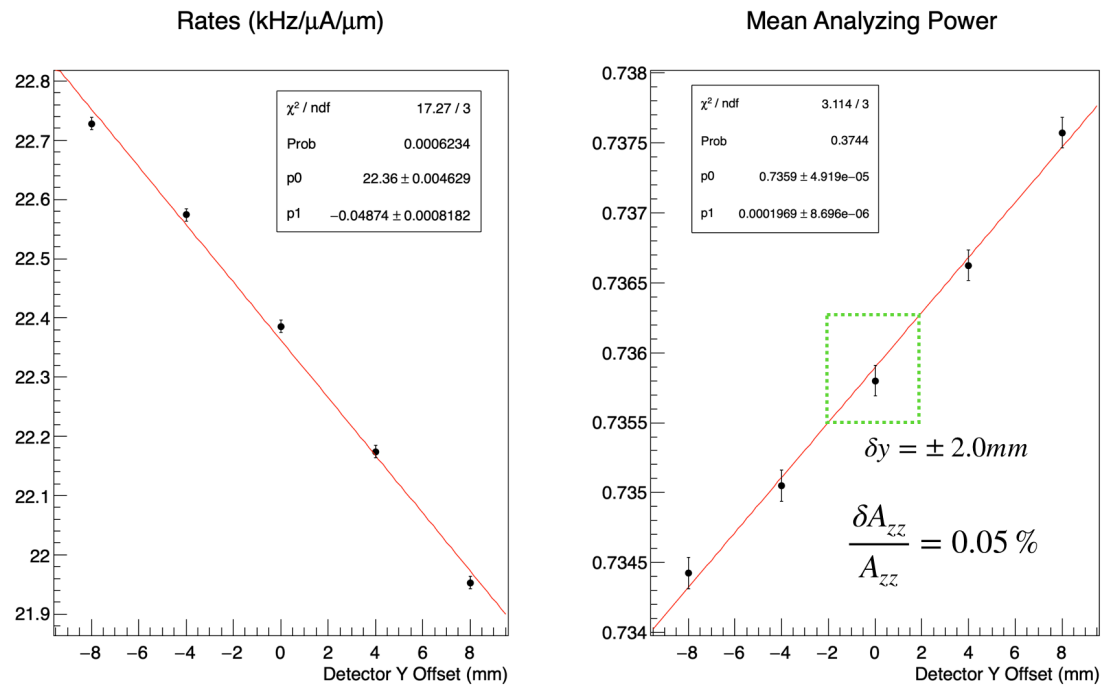

Figure 4.31: y detector position dependence on $\left\langle A_{z z}\right\rangle$ and rates. The width of the green box represents the uncertainty of the $\mathrm{x}$-axis and the height the corresponding uncertainty of $\left\langle A_{z z}\right\rangle$.
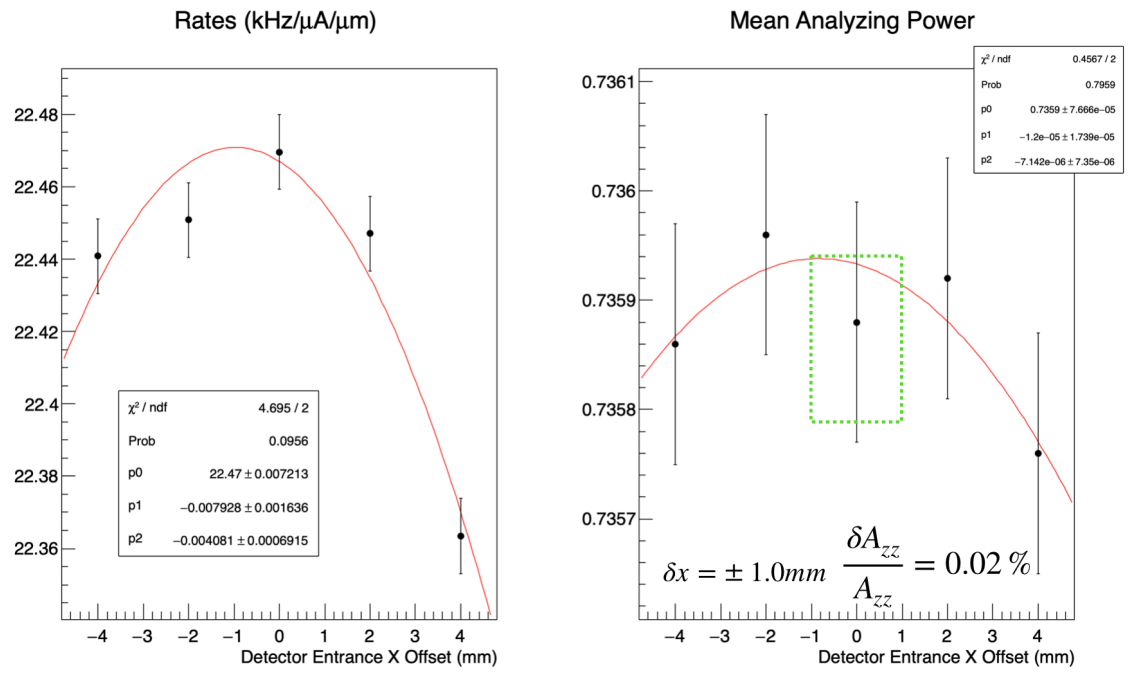

Figure 4.32: $x$ detector entrance position dependence on $\left\langle A_{z z}\right\rangle$ and rates. The width of the green box represents the uncertainty of the $\mathrm{x}$-axis and the height the corresponding uncertainty of $\left\langle A_{z z}\right\rangle$. 

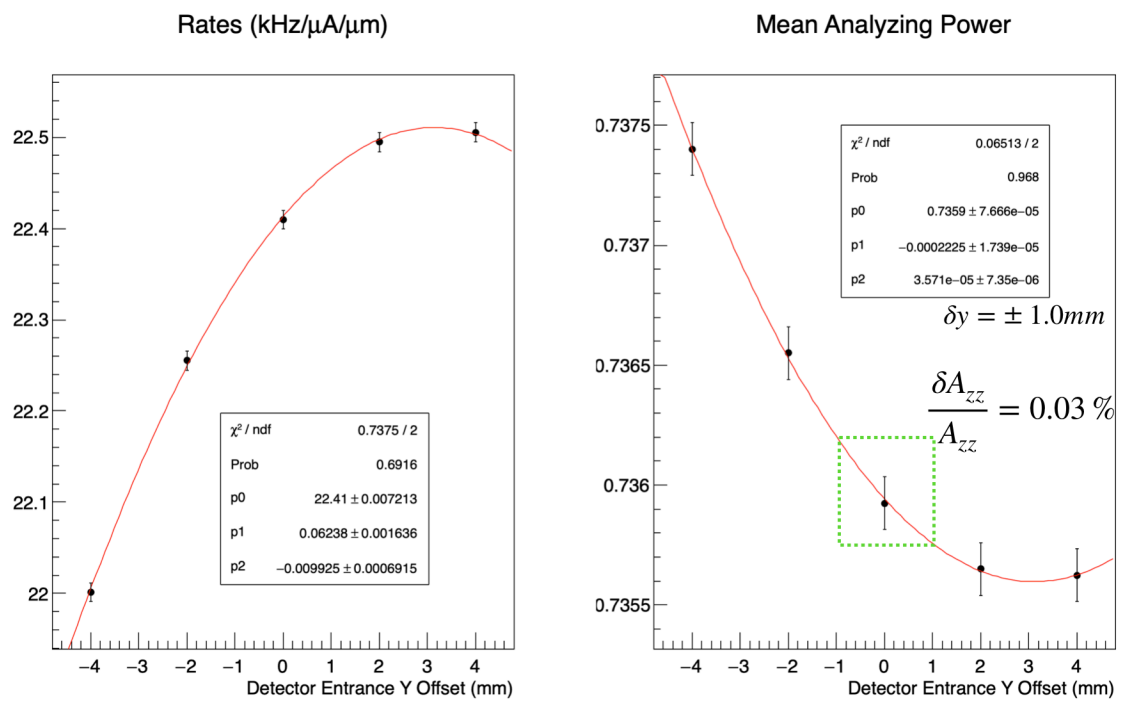

Figure 4.33: y detector entrance position dependence on $\left\langle A_{z z}\right\rangle$ and rates. The width of the green box represents the uncertainty of the $\mathrm{x}$-axis and the height the corresponding uncertainty of $\left\langle A_{z z}\right\rangle$. 


\subsubsection{Collimator}

The movable collimators located at the dipole entrance are sealed within the dipole vacuum box. Two viewports enable the collimator position to be visually inspected. The vertical gap of the collimator is adjusted by means of a manual operated dial (initially a stepping motor controlled the motion but currently it is not in use). The opening of the collimator now is estimated to be $3.6 \mathrm{~cm}$ which was estimated from photos and the known inner radius of the viewport. The sensitivity of $\left\langle A_{z z}\right\rangle$ is shown in Figure 4.34. An uncertainty in the opening of $2 \mathrm{~mm}$ corresponds to an uncertainty in $\left\langle A_{z z}\right\rangle$ of $0.01 \%$.
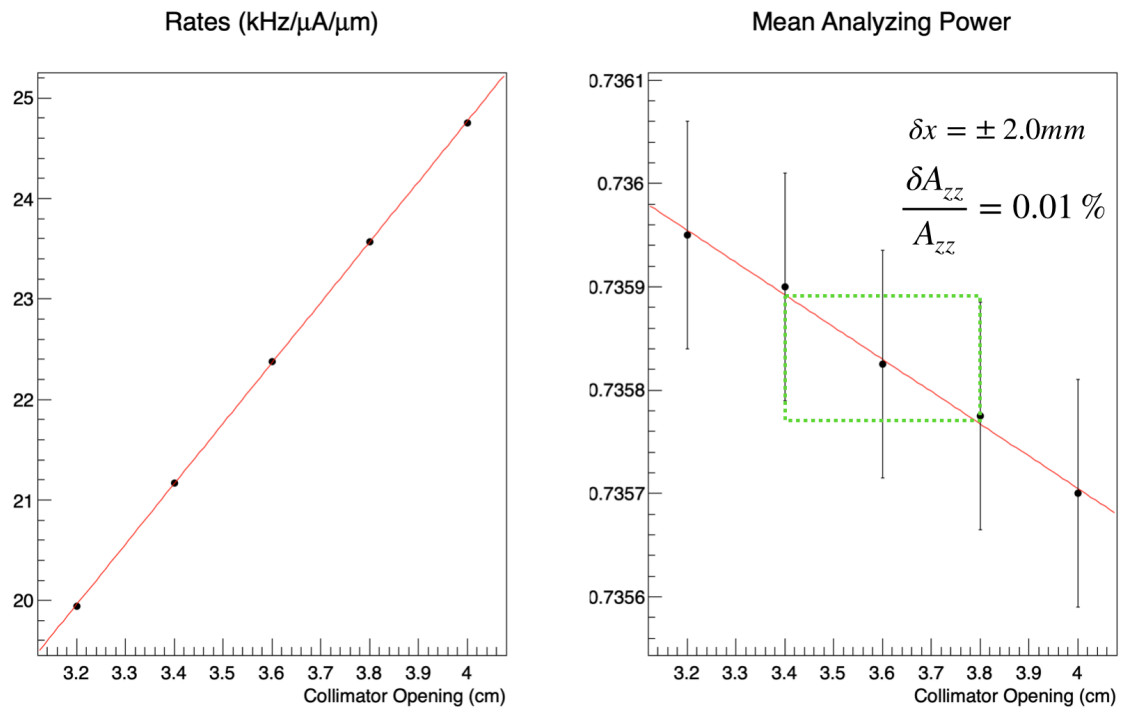

Figure 4.34: Collimator opening dependence on $\left\langle A_{z z}\right\rangle$ and rates. The width of the green box represents the uncertainty of the $\mathrm{x}$-axis and the height the corresponding uncertainty of $\left\langle A_{z z}\right\rangle$.

\subsubsection{Multiple Scattering}

Multiple scattering in the target foil can alter the angle which the electrons exit. This effect was studied by altering the foil thicknesses and by turning on and off the effect in the simulation. Figure 4.35 shows only slight differences in $\left\langle A_{z z}\right\rangle$ for different foil thicknesses and therefore the effect can be neglected.

\subsubsection{Magnetic Fields and Alignment}

Geant 4 studies of the split coil magnet alignment were performed by offsetting the magnet $1 \mathrm{~mm}$ or 1 milliradian $(\mathrm{mr})$ in the $\mathrm{x}$ or $\mathrm{y}$ directions at $2.2 \mathrm{GeV}$ and $0.95 \mathrm{GeV}$. The expected 

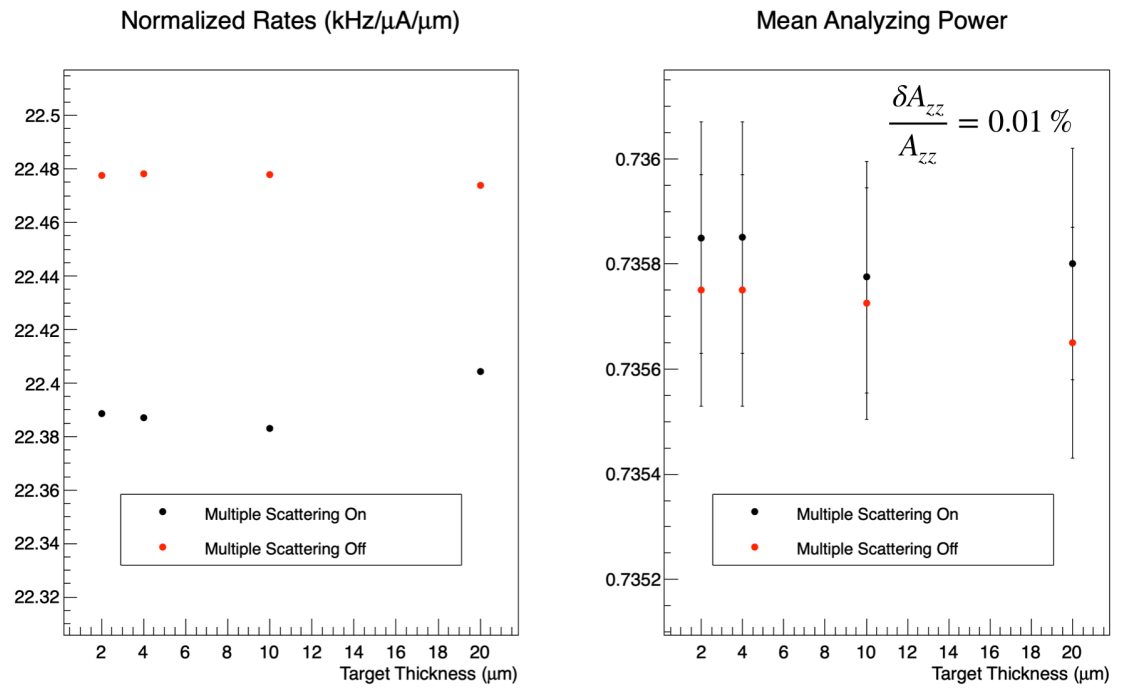

Figure 4.35: The effects of multiple scattering for different foil thickness on $\left\langle A_{z z}\right\rangle$ and rates.

tolerance for the $\mathrm{x} / \mathrm{y}$ offsets are at the $0.1 \mathrm{~mm}$ level and for the angular allignment a few mr. For both beam energies the combined change in $\left\langle A_{z z}\right\rangle$ for a $1 \mathrm{~mm} \mathrm{x}$ and y shift was $\sim 0.15 \%$, here the individual $\mathrm{x}$ and $\mathrm{y}$ shifts are added in quadrature. Similarly for both beam energies the angular misalignment resulted in a change in $\left\langle A_{z z}\right\rangle$ of $0.02 \%$.

The current in each magnet is known within $2 \%$. The accuracy of the model that translates these currents into actual fields is the subject of an ongoing study. In another Geant4 study the fields of the split coil magnet, quadrupoles and dipole were adjusted by $2 \%$ and the change in $\left\langle A_{z z}\right\rangle$ was reported. There was a $0.02 \%$ effect for all the magnets with the exception of the dipole. A $2 \%$ change in the field of the dipole leads to a $0.2 \%$ change in $\left\langle A_{z z}\right\rangle$. 


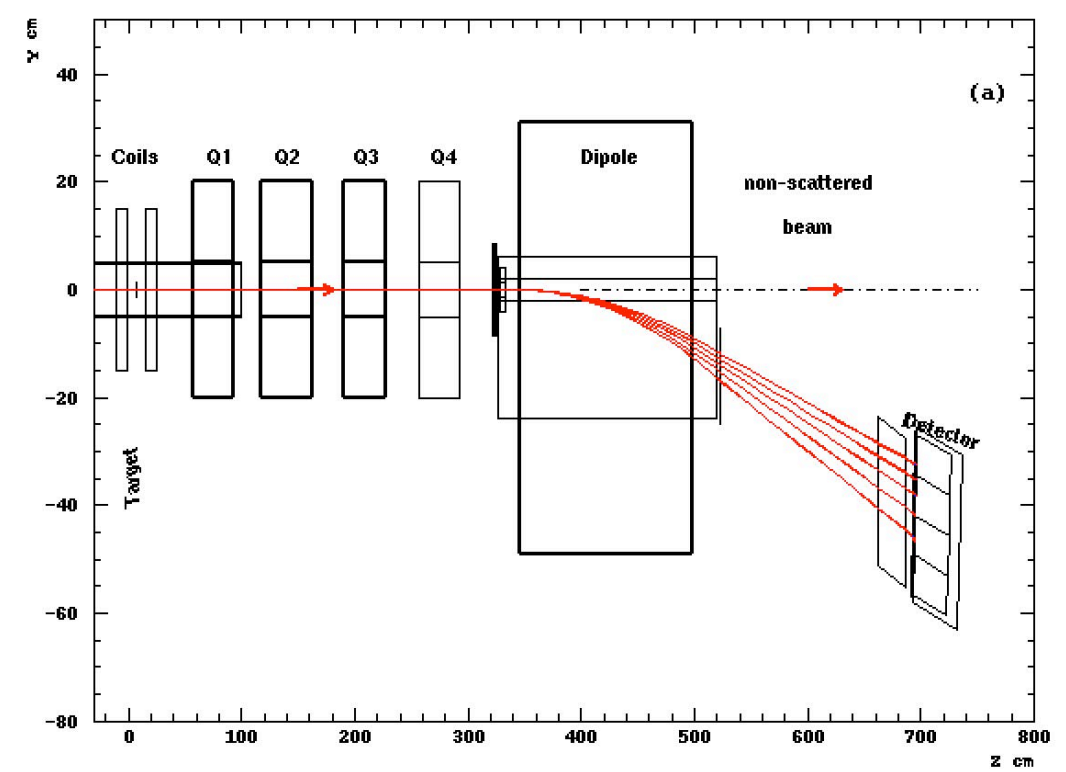

Figure 4.36: Key elements in Geant3 simulation of Møller Polarimeter

\subsection{Radiative Corrections}

Radiative corrections to the analyzing power have been calculated[74, 75], and are expected to reduce the analyzing power in the JLab and SLAC (45GeV) Møller polarimeters by $0.2 \%$ to $1.0 \%$ [6]. The Hall A polarimeter has reported systematic errors of $0.3 \%$ due to radiative corrections; however in the past Geant 3 did not have the corrections included in the simulations. The new Geant 4 simulation includes radiative effects and the correction at $2.2 \mathrm{GeV}$ is $\sim 0.3 \%$. Therefore assuming the simulation is accurate to within $33 \%$ we can assign an uncertainty to the correction of $0.1 \%$. This uncertainty can be reduced as the new simulation package is validated and further investigations are made.

\subsection{Levchuk Effect}

When determining the systematic errors associated with Møller polarimetry it is important to considerer the motion of the target electrons. The target electrons being free and motionless is an idealization, and therefore a theory is needed in order to account for the effects of scattering off of a moving target. The importance of this effect was discovered over 25 years ago by L.G. Levchuk and is known as the Levchuk effect. He showed that the 
effect led to a $\sim 10 \%$ correction to the MIT-Bates Møller polarimeter measurements[56]. In his paper he detailed the kinematics of Møller scattering when taking into account the intra-atomic Fermi motion of the target electrons. Shorty afterwards, Swartz et al. applied the Levchuk effect to the Møller polarimeter at the SLAC linear collider and showed it was a $14 \%$ effect[57]. It was determined that the Levchuk Effect could be minimized by engineering polarimeters which featured a large $\theta_{C M}$ acceptance, which was one of the goals behind the design of the Hall A Moller polarimeter at JLab[76].

To appreciate the significance of the Levchuk effect let us first approximate the Møller laboratory scattering angle, $\theta_{L a b}$, as was shown in [57]. The exact relationship between $\theta_{L a b}$ and the center-of-mass scattering angle $\theta_{C M}$ is

$$
\tan \theta_{L a b}=\sqrt{\frac{2 m_{e}}{E_{0}+m_{e}}} \frac{\sin \theta_{C M}}{1+\cos \theta_{C M}}
$$

where $E_{0}$ is the energy of the incident (beam) electron and $m_{e}$ is the mass of the electron. The scattered electron's momentum, $p^{\prime}$ is

$$
p^{\prime}=\frac{p_{B}}{2}\left(1+\cos \theta_{C M}\right)
$$

where $p_{B}$ is the momentum of the incident electron. Using the small angle approximation for $\theta_{L a b}$, and assuming the target is at rest, such that the center-of-mass energy is $s_{0}=2 p_{B} m_{e}$ then Equation 4.35 can be written as

$$
\theta_{\text {Lab }}^{2}=\frac{1}{p^{\prime} p_{B}} \frac{s_{0}}{2}\left(1-\cos \theta_{C M}\right)=2 m_{e}\left(\frac{1}{p^{\prime}}-\frac{1}{p_{B}}\right)
$$

However, if the motion of the target electron is considered then the center-of-mass energy, $s_{1}$, is

$$
s_{1}=s_{0}\left(1-\frac{\mathbf{p}_{\mathbf{t}} \cdot \hat{\mathbf{n}}}{m_{e}}\right)
$$

where $\mathbf{p}_{\mathbf{t}}$ is the target momentum and $\hat{\mathbf{n}}$ is the direction of the incident beam momentum. Equation 4.35 is still valid for non-zero target momentum but Equation 4.36 becomes

$$
\theta_{\text {Lab }}^{2}=\frac{1}{p^{\prime} p_{B}} \frac{s_{1}}{2}\left(1-\cos \theta_{C M}\right)=2 m_{e}\left(\frac{1}{p^{\prime}}-\frac{1}{p_{B}}\right)\left(1-\frac{\mathbf{p}_{\mathbf{t}} \cdot \hat{\mathbf{n}}}{m_{e}}\right)
$$

This derivation follows the analysis in [57]. Equation 4.38 is the basis of the Levchuk effect. The intra-atomic Fermi motion of the target electrons causes the laboratory scatter- 
ing angle to be smeared by the factor $\left(1-\frac{\mathbf{p}_{\mathbf{t}} \cdot \hat{\mathbf{n}}}{m_{e}}\right)$. Of the 26 electrons found in Fe, two are in the K-shell, eight in the L-shell, and 16 in the outer $\mathrm{M}$ and $\mathrm{N}$ shells with mean momenta $\sim 90 \mathrm{keV} / \mathrm{c}, \sim 30 \mathrm{keV} / \mathrm{c}, \sim 10 \mathrm{keV} / \mathrm{c}$, and $\sim 2 \mathrm{keV} / \mathrm{c}$ respectively. For inner most K-shell electrons, the angular smearing can be as large as an $18 \%$ effect $\left(1-\frac{90}{511}\right)$ when the target momentum is parallel or anti-parallel to the beam direction.

The Levchuk effect can be greatly reduced by increasing the acceptance or decreasing the energy granularity of the detector. The apparatus in Hall A features a larger acceptance $\left(> \pm 10^{\circ}\right)$ than the early polarimeters at SLAC and MIT-BATES. Those early polarimeters were also single arm meaning they only detected one of the two scattered electrons for each Møller event. This technique requires having a segmented strip detectors to provide energy resolution (the strip number is correlated to the energy of the electron) which allows for the background from Mott (electron-nucleus) scattering to be supressed. This is because Mott electrons have an energy of $\sim E_{\text {Beam }}$ while Møller electrons have an energy of $\sim E_{\text {Beam }} / 2$. High granularity detectors like this are more sensitive to the smearing of the Levchuk effect. The Hall A polarimeter uses two arms and detects both Møller electron pairs in coincidence which allows for a low granularity detector since the timing information allows for background suppression.

Figure 4.37 shows a top view of the Hall A polarimeter and the path of $\theta_{C M}=90^{\circ}$ Møller scattered electrons for the K, L, and M-shell. From the figure one can see how the trajectory (red lines) of the particles are smeared by the Levchuk effect. The detector acceptance for outer shell electrons is larger than the inner shells. This leads to an increase of the effective target polarization since polarized outer shell electrons have a better chance of reaching the detector.

To calculate the correction in Geant3, the simulation is run three times, once for the $\mathrm{K}$, L, and M-shells. The outer M-shell electrons are treated as being free electrons (no target momentum), while the inner shells have the randomly-oriented momentum distributions of the target electrons included. Only the outer shell electrons are polarized, so a relative difference in the acceptance between inner and outer shell scattering events requires a correction to the theoretical total polarization of the atom. If the inner shell electrons, for example are more likely to scatter out of the acceptance due to the energy smearing, their absence will increase the effective target polarization. As an example, suppose the simulation was run for each shell and 100,000 events were generated each time. Of those 100,000 electron pairs, suppose 25,000 M-Shell coincidence pairs reached the detector while the $\mathrm{L}$ 


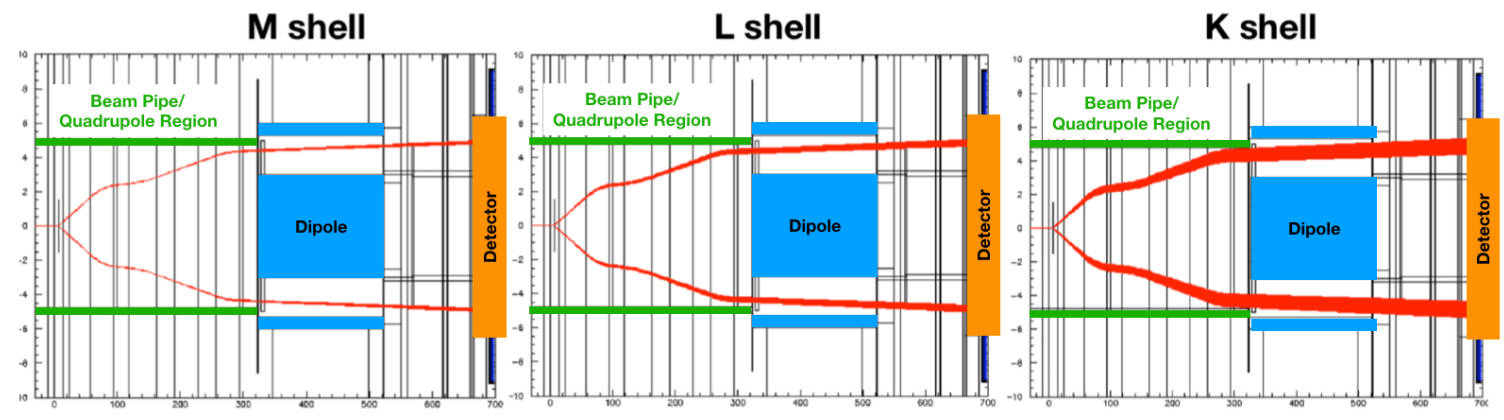

Figure 4.37: $\theta_{C M}=90^{\circ}$ Møller scattered electron trajectories when scattered from different atomic shells in the Hall A polarimeter. The inner shells tend to smear the angular distribution resulting in an increase of the effective target polarization.

and K-shells had 24,000 and 22,000 pairs respectively. The size of the correction can be approximated as

$$
\frac{26 N_{M}}{2 N_{K}+8 N_{L}+10 N_{M}}=\frac{26(2500)}{2(22,000)+8(24,000)+16(25,000)}=1.02
$$

or a $2 \%$ correction.

The Hall A polarimeter typically has Levchuk corrections at the few percent level. The correction depends on the beam energy and the acceptance of the detector which is determined from the magnet settings. The correction for one $2.2 \mathrm{GeV}$ optical configuration is $0.37 \% \pm 0.15 \%$ (stat.) when the system is tuned. The sign of the correction is usually positive, which means it increases the effective target polarization thereby increasing the measured asymmetry. However at certain magnet settings, when the system is not tuned so that the rates are not optimized, the inner shell electrons can have a higher probability of reaching the detector which has the opposite effect (decreases the effective target polarization). This behavior provides a unique method to test the size of the correction and should be considered in future studies. 
Table 4.4: Error Budget Table for Møller Polarimetry at $2.2 \mathrm{GeV}$

\begin{tabular}{||lcc||}
\hline Source & Uncertainity & Effect on $\frac{\delta P_{\text {Beam }}}{P_{\text {Beam }}}$ \\
\hline \hline Target Polarization & $0.30 \%$ & $0.30 \%$ \\
\hline Beam Position (x) & $0.2 \mathrm{~mm}$ & $0.05 \%$ \\
\hline Beam Position (y) & $0.2 \mathrm{~mm}$ & $0.08 \%$ \\
\hline Beam Energy & $2.5 \times 10^{-4}$ & $0.01 \%$ \\
\hline Detector Position (x) & $2 \mathrm{~mm}$ & $0.01 \%$ \\
\hline Detector Position (y) & $2 \mathrm{~mm}$ & $0.05 \%$ \\
\hline Detector Entrance Position (x) & $1 \mathrm{~mm}$ & $0.02 \%$ \\
\hline Detector Entrance Position (y) & $1 \mathrm{~mm}$ & $0.03 \%$ \\
\hline Collimator Opening & $2 \mathrm{~mm}$ & $0.01 \%$ \\
\hline Split Coil Magnet Position (x/y) & $1 \mathrm{~mm}$ & $0.15 \%$ \\
\hline Split Coil Field & $2 \%$ & $0.02 \%$ \\
\hline Q1 Field & $2 \%$ & $0.02 \%$ \\
\hline Q2 Field & $2 \%$ & $0.02 \%$ \\
\hline Q4 Field & $2 \%$ & $0.02 \%$ \\
\hline Dipole Field & $2 \%$ & $0.20 \%$ \\
\hline Levchuk Effect & $50 \%$ & $0.20 \%$ \\
\hline Deadtime & $33 \%$ & $0.10 \%$ \\
\hline Radiative Corrections & $33 \%$ & $0.10 \%$ \\
\hline Total & & $0.47 \%$ \\
\hline
\end{tabular}




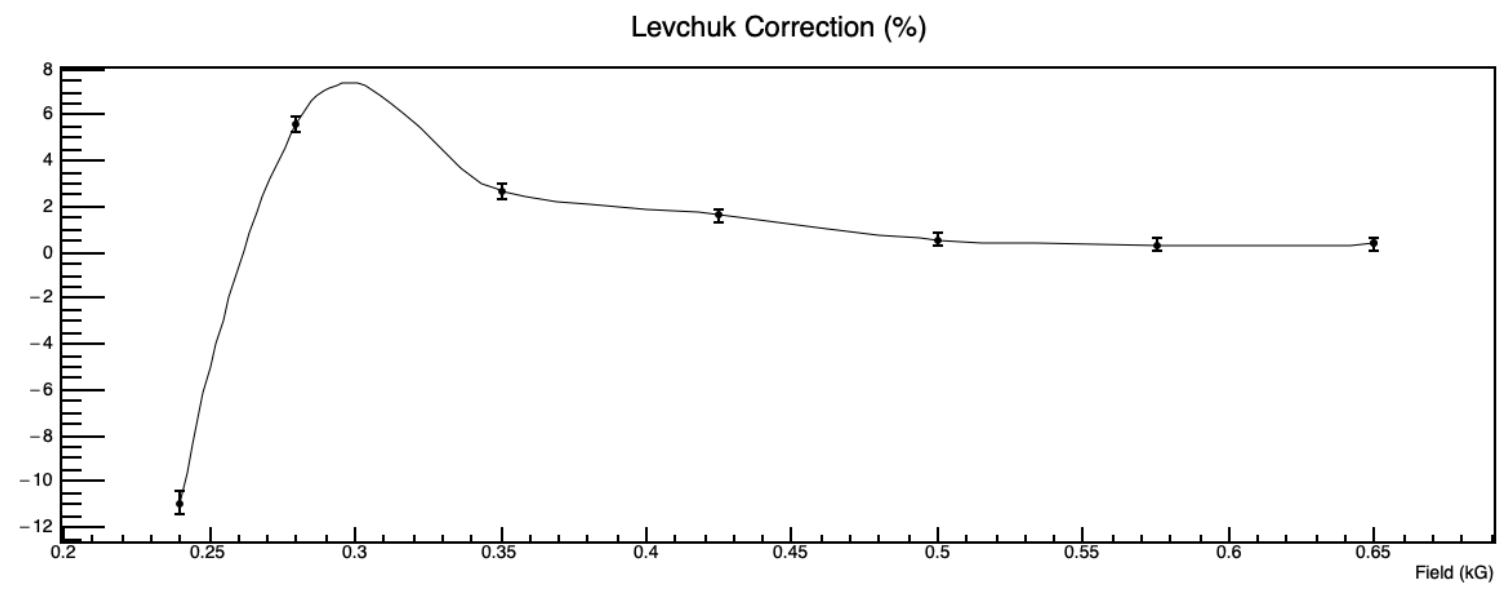

Figure 4.38: The Levchuk correction as a function of the magnetic field of Q2. This particular tune is for a $2.1 \mathrm{GeV}$ beam. The structure of the correction at the lower fields can provide a check for our understanding of the effect. 


\section{CHAPTER 5}

\section{POLARIZATION MEASUREMENTS AFTER THE UPGRADE}

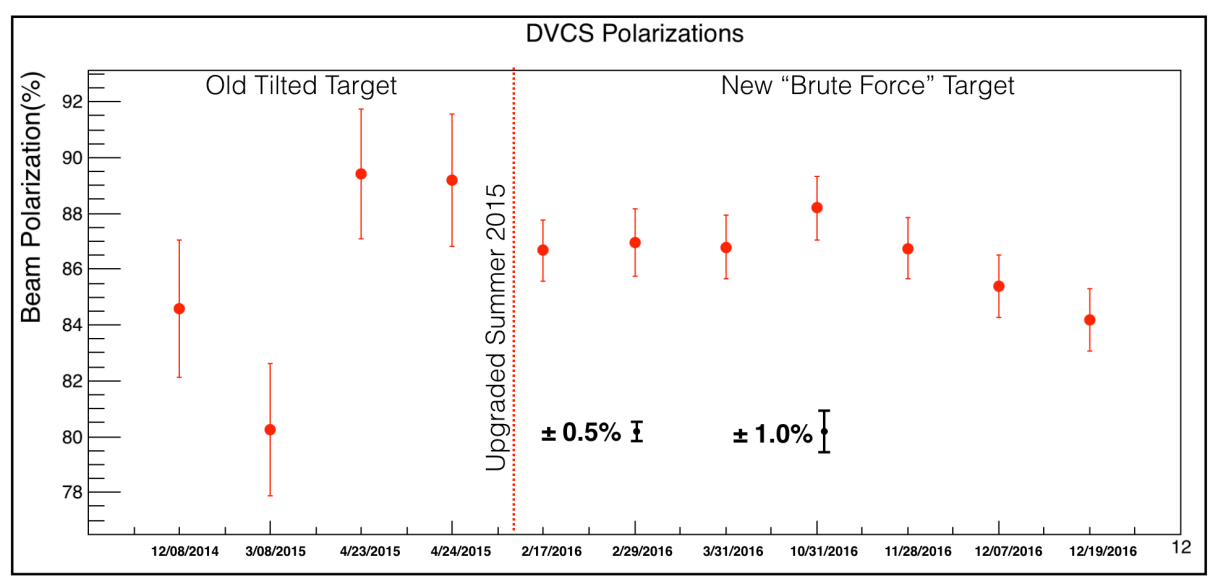

Figure 5.1: Beam polarization measurements before and after the upgrade of the Hall A.

\subsection{DVCS Results}

The DVCS experiment (E12-06-114), which ran in Hall A from 2014 to 2016, required percent level polarimetry. The experiment began with the Møller polarimeter using the low-field tilted target. In the summer of 2015, the system was upgraded with the new superconducting split coil magnet and Temple University target motion system. The high 
field target was used for the remainder of the experiment which allowed the overall systematic error to be reduce by more than a factor of 2 (See Figure 5.2). In addition to providing numerous polarization measurements, a limited number of systematic test were performed during each measurement.

\begin{tabular}{|l|c|c|}
\hline \multicolumn{1}{|c|}{ Variable } & Tilted & Brute force \\
\hline Target polarization & $2.0 \%$ & $0.5 \%$ \\
\hline GEANT & $0.5 \%$ & $0.5 \%$ \\
\hline Target temperature & $0.2 \%$ & $0.2 \%$ \\
\hline Dead time & $0.05 \%$ & $0.05 \%$ \\
\hline Background & $0.3 \%$ & $0.3 \%$ \\
\hline High beam current & $0.2 \%$ & $0.2 \%$ \\
\hline Stability & $0.5 \%$ & $0.5 \%$ \\
\hline Total: & $2.2 \%$ & $1.0 \%$ \\
\hline
\end{tabular}

Figure 5.2: The Møller polarimetry systematic errors during E12-06-114 which used the tilted and brute-force target.

The final beam polarization results for the DVCS experiment are shown in Figure 5.1. The systematic errors, reflected in the size of the error bars, were reduced after the upgrade as a result of the smaller target polarization uncertainty with the brute-force target. Polarization measurements with the new target were done at beam energies of 4.4, 8.8, and $11 \mathrm{GeV}$. The results shown include a Levchuk effect and dead time correction. The rates were normalized to beam current and accidental coincidence subtraction was applied.

One of the additional systematic tests performed during DVCS was a "mini-spin dance". The orientation of the beam polarization is set at the source by a device called a Wein filter. Here the Wein angle is set, which corresponds to the direction that the spins are oriented before they are accelerated. As the electron's are bent around the racetrack design of the facility they undergo spin precession. The size of the precession depends on the beam energy and the number of times that they go around the track, it is on the order of thousands of a degree. Therefore the Wein angle at the source must be carefully set in order to maximize the longitudinal polarization delivered to the experimental halls. This test is called a "mini-spin dance" because a normal "spin dance" involves multiple experimental halls doing independent beam polarization measurements simultaneously as a cross check 
of the individual polarimeters. Figure 5.3 are the results from the "Mini-spin dance". The magnitude of the longitudinal polarization is a maximum for a Wein angle of $\sim\left(-92.2^{\circ}\right)$.

Beam Polarization vs Wein Angle at $8.824 \mathrm{GeV}$ on Feb. 29th, 2016

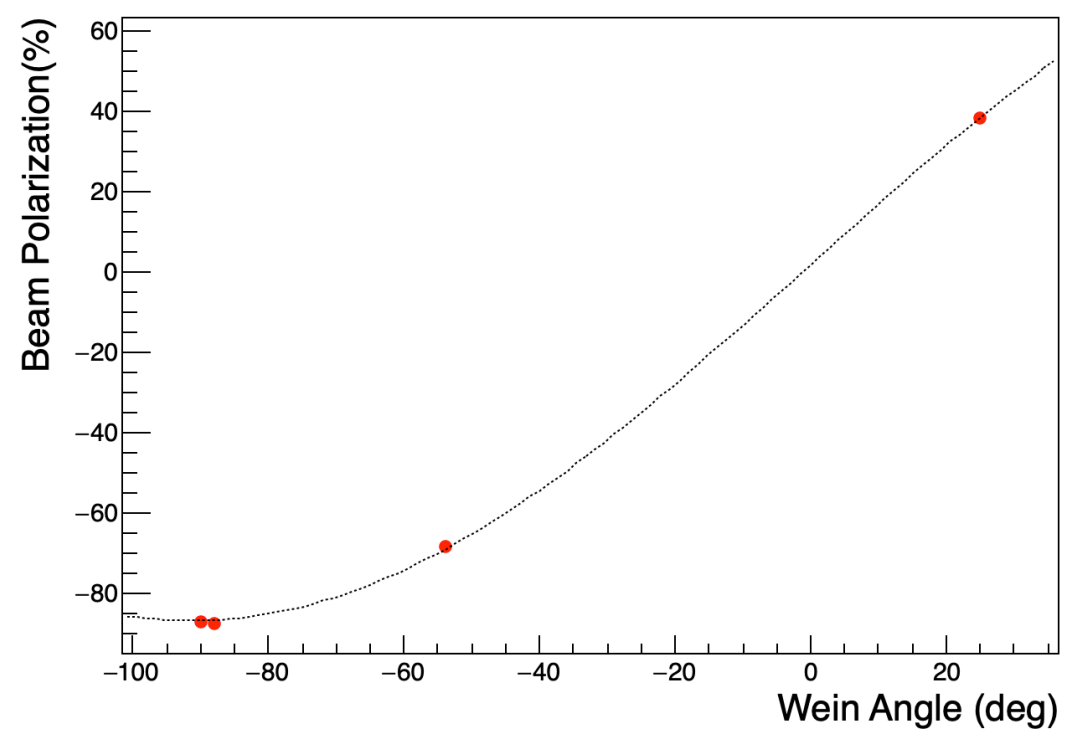

Figure 5.3: Measured longitudinal polarization delivered to Hall $A$ as a function of Wein angle set at source

In order to verify target alignment, a saturation test was performed by varying the split coil magnetic field. If the target is close to $90^{\circ}$ then according to the Stoner Wolfarth model, fields from $3 \mathrm{~T}$ to $4 \mathrm{~T}$ should yield the same result. Figure 5.4 shows the results from the saturation test. The error bars only include statistical uncertainties, and are relatively large because the data runs were short due to limited beam time. Therefore it is difficult to extract a target angle but the result does indicate that the target was at or close to saturation since, within statistics, the measured polarization remains constant.

Quadrupole scans were performed on six separate occasions in 2016. The scans are used as a crosscheck of the simulations since different optical configurations yield different analyzing powers and Levchuk corrections. Ideally the different configurations would provide the same beam polarization result when the correct analyzing power and Levchuk corrections are applied. The results of the scans are shown in Figure 5.5. The scans in March, April and November, within statistics, yield approximately the same result. However the remaining scans present some concerns because the point to point differences are as large as $1.6 \%$. 
Beam Polarization vs Field Strength at $4.47 \mathrm{GeV}$ on Feb. 17, 2016

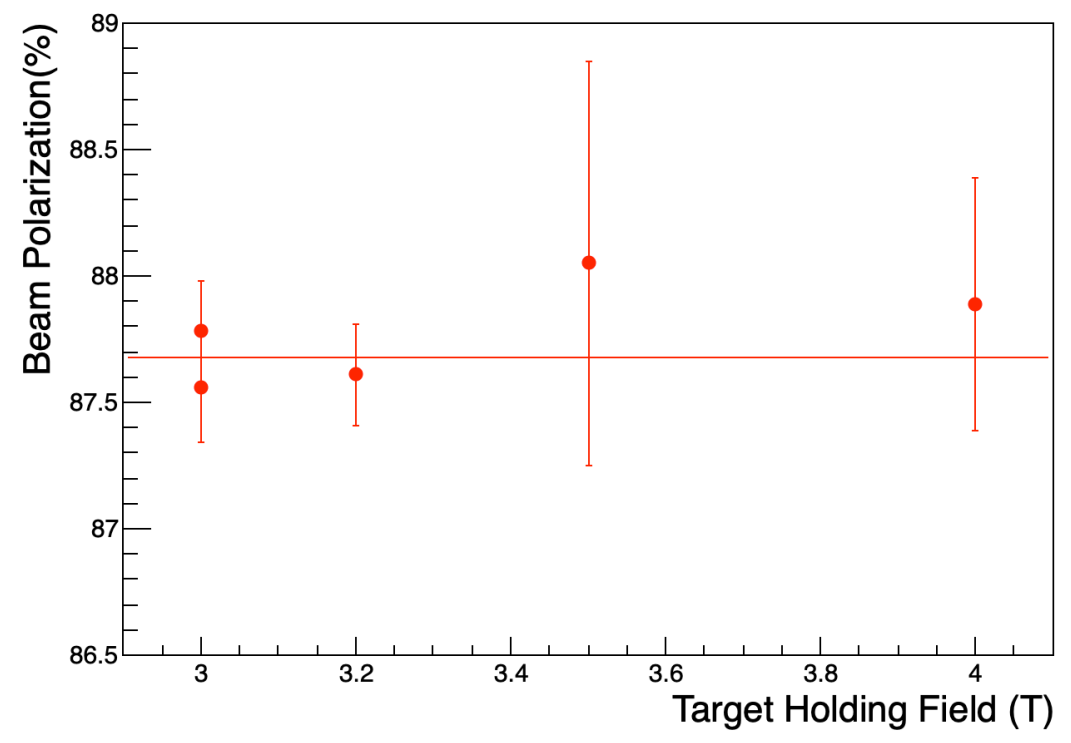

Figure 5.4: Measured polarization as a function of the target holding field. Ideally the target foil would be at exactly $90^{\circ}$ and the foil would reach maximum polarization at $2.2 \mathrm{~T}$. 


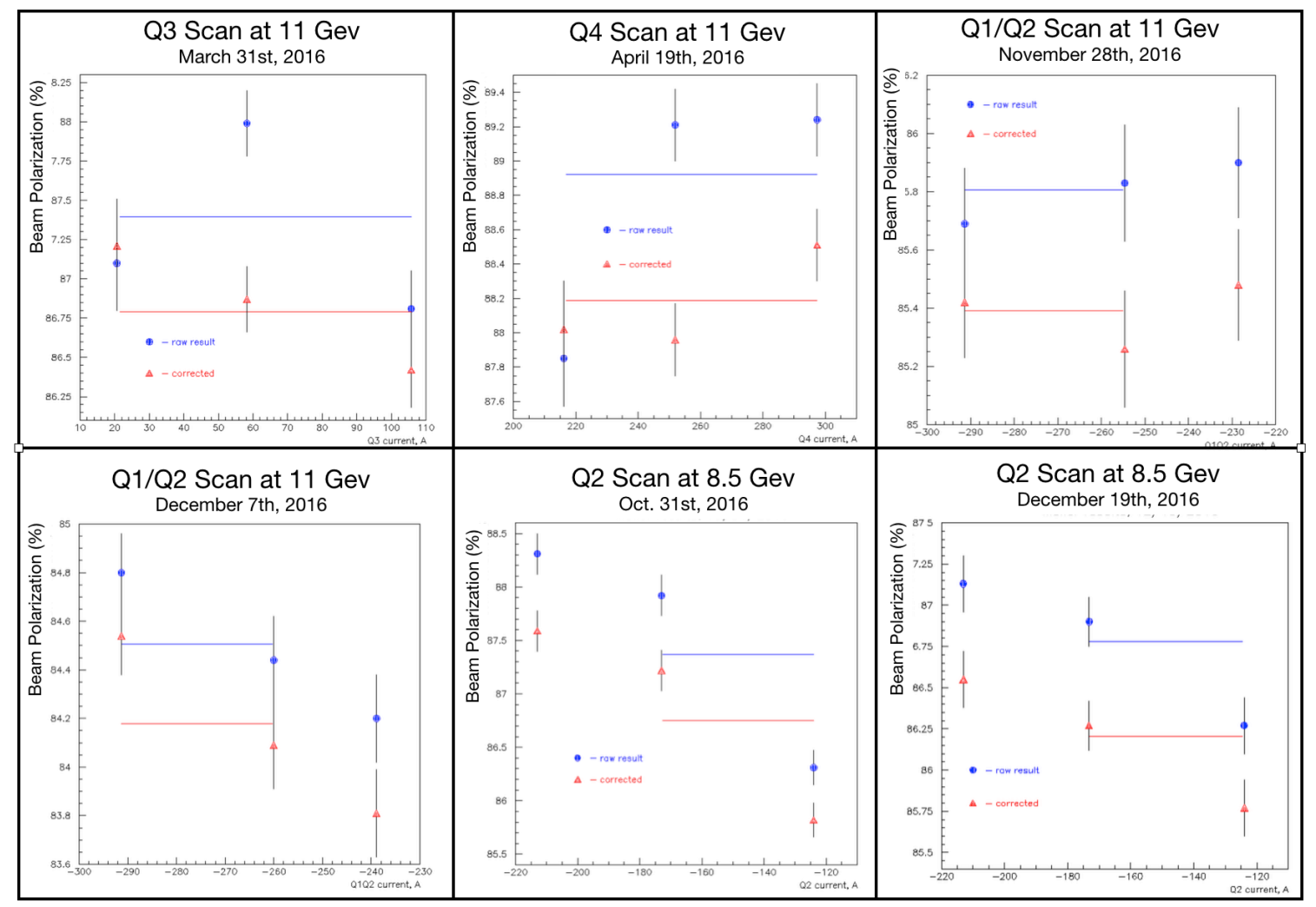

Figure 5.5: Quad scans performed in 2016. The red triangles have been corrected for the Levchuk effect. 


\subsection{Commisioning Run}

The new Møller polarimeter was commissioned March 18-19, 2019 with a beam energy of $2.14 \mathrm{GeV}$. During the two days of dedicated beam time several tests were performed which included target centering tests, target angle scans, polarization measurements with two different CREX optics solutions, and quadrupole field rate scans. The commission was done in anticipation of the PREX/CREX experimental program which is scheduled to commence in the summer of 2019. The preliminary results are presented here.

A summary of the polarization and asymmetry measurements are shown in Figure 5.6. Since all of the measurements were taken with the same magnet settings the asymmetries and polarization are directly proportional to each other. The data taken with the $1 \mu \mathrm{m}$ foil is statistically lower than the data taken with the thicker foils. The likely explanation for this is because of known wrinkles in the $1 \mu \mathrm{m}$ which could cause the target angle to be misaligned by several degrees. Additional tests to validate this explanation could include increasing the target magnetic field to overcome the misalignment or moving the position of the target so that the beam would be incident on a wrinkle free section of the foil. The spread of the measurements with the $1 \mu \mathrm{m}$ foil are also a concern. Specifically the first three polarization measurements which were taken consecutively with no changes to the apparatus being made. A closer look at the data is shown in Figure 5.7. The plot on the top show the rates for the two different beam helicity states averaged every 4 seconds, after they have been normalized for beam current fluctuations and accidental subtraction (therefore the units are somewhat arbitrary). The bottom plot is the asymmetry, also averaged every 4 seconds. Current analysis efforts are searching for a correlation between the drifting seen in the data and other beam parameters such as the beam position, current or energy stability. The polarization measurements for the $4 \mu \mathrm{m}$ and $10 \mu \mathrm{m}$ are also shown in Figure 5.6. The point to point differences here are also somewhat concerning but not as dramatic as the $1 \mu \mathrm{m}$ foil. The measurements made at $2.5 \mathrm{~T}$ were part of the target angle scans so the target was rotated between each measurement so there is no guarantee that the foil was at exactly the same target angle for each run. After the analysis it was learned that the target angle read back values were off by more than $1^{\circ}$. This came as a surprise since the system had been extensively tested and shown to have sub $0.1^{\circ}$ precision when controlled with an Arduino micro controller. Currently the system is being troubleshooted in an attempt to restore the desired angular precision. 


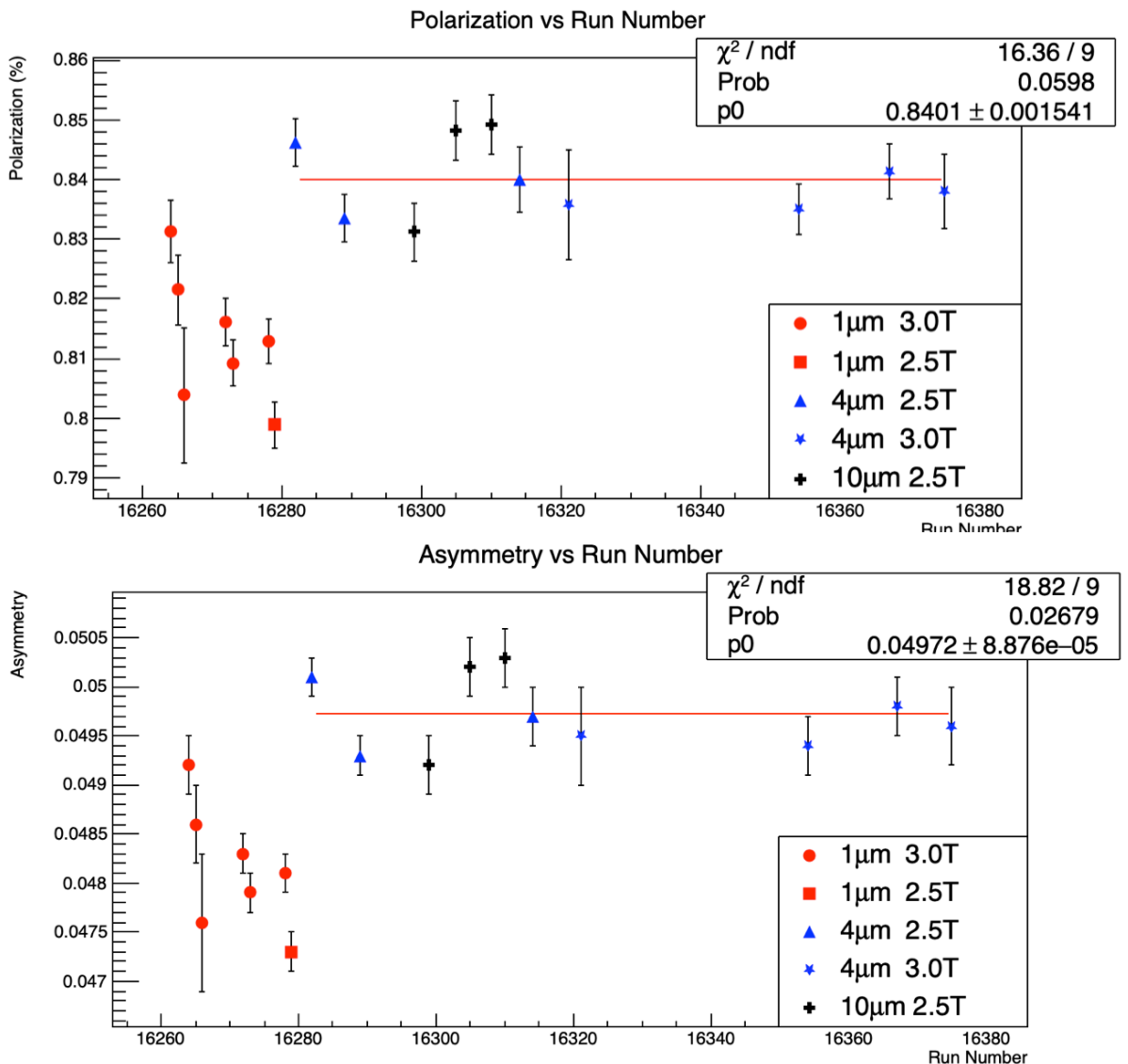

Figure 5.6: Polarization measurements during 2019 commissioning. The statistically lower polarization measured with the $1 \mu \mathrm{m}$ foil could be a result of wrinkles or deformations in the foil. 


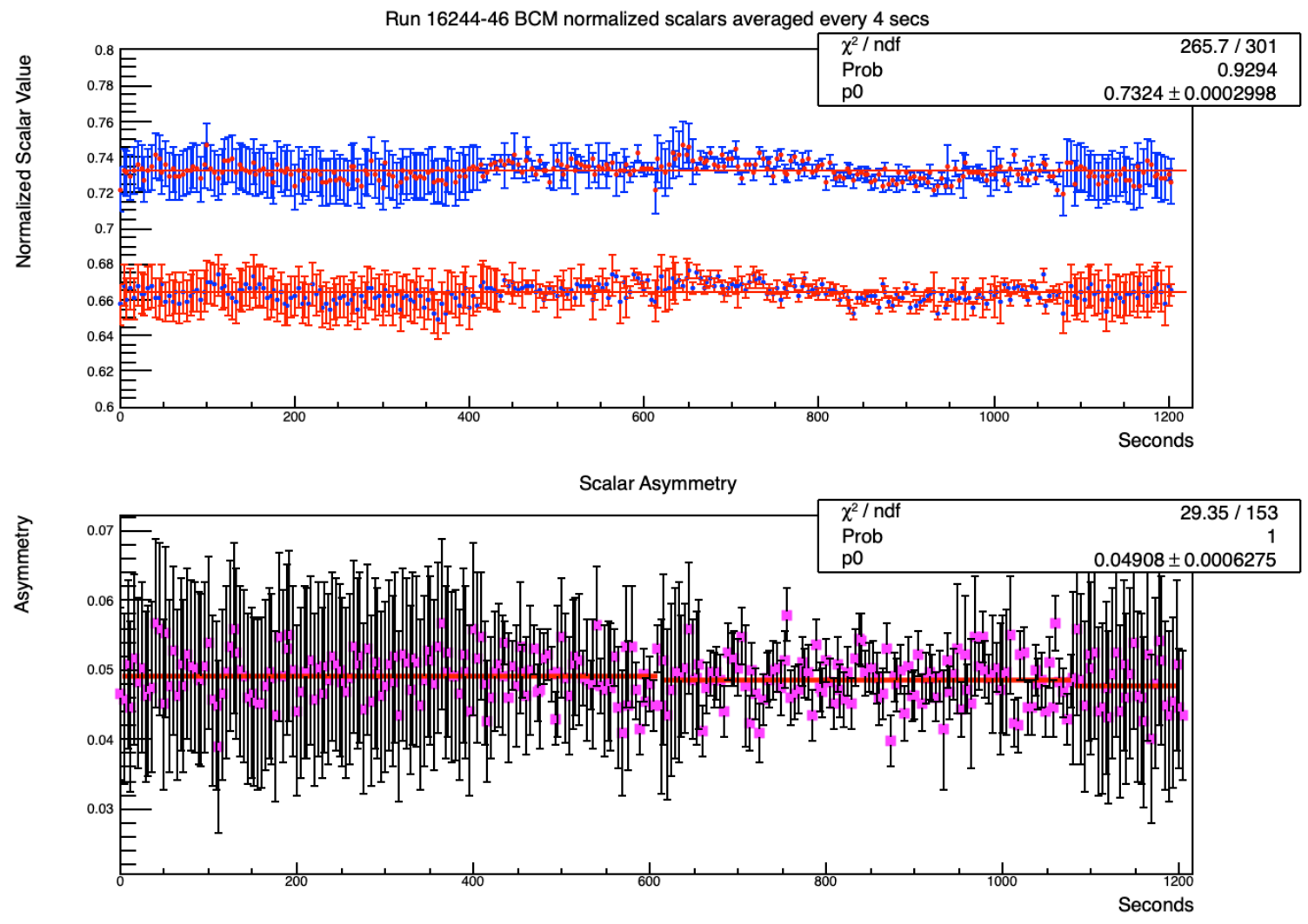

Figure 5.7: (Top)Current normalized scalars averaged every 4 seconds for both helicity cycles. (Bottom) Asymmetry averaged every 4 seconds ( $30 \mathrm{~Hz}$ helicity flip rate) 

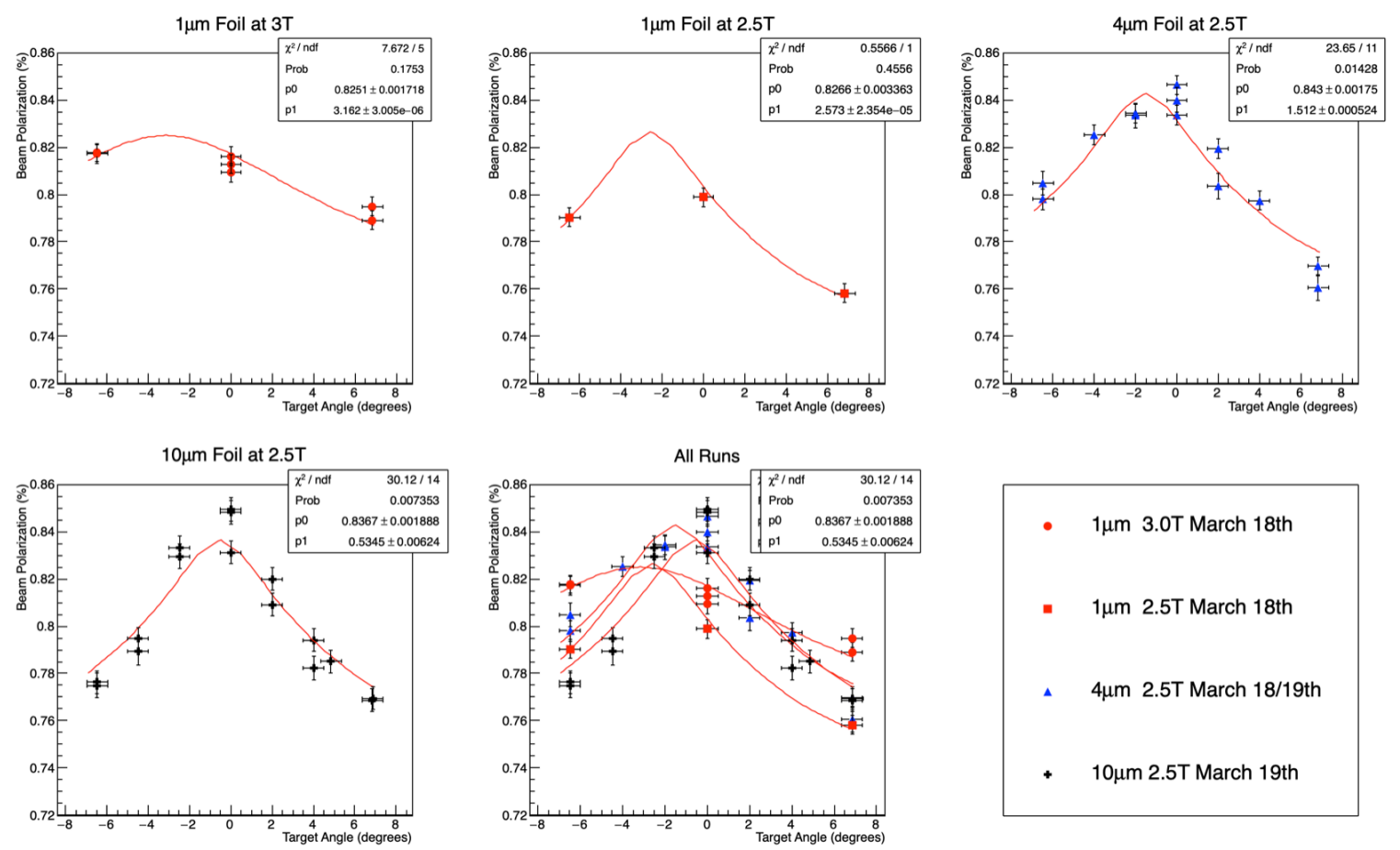

Figure 5.8: Polarization measurements while changing the target foil angle 


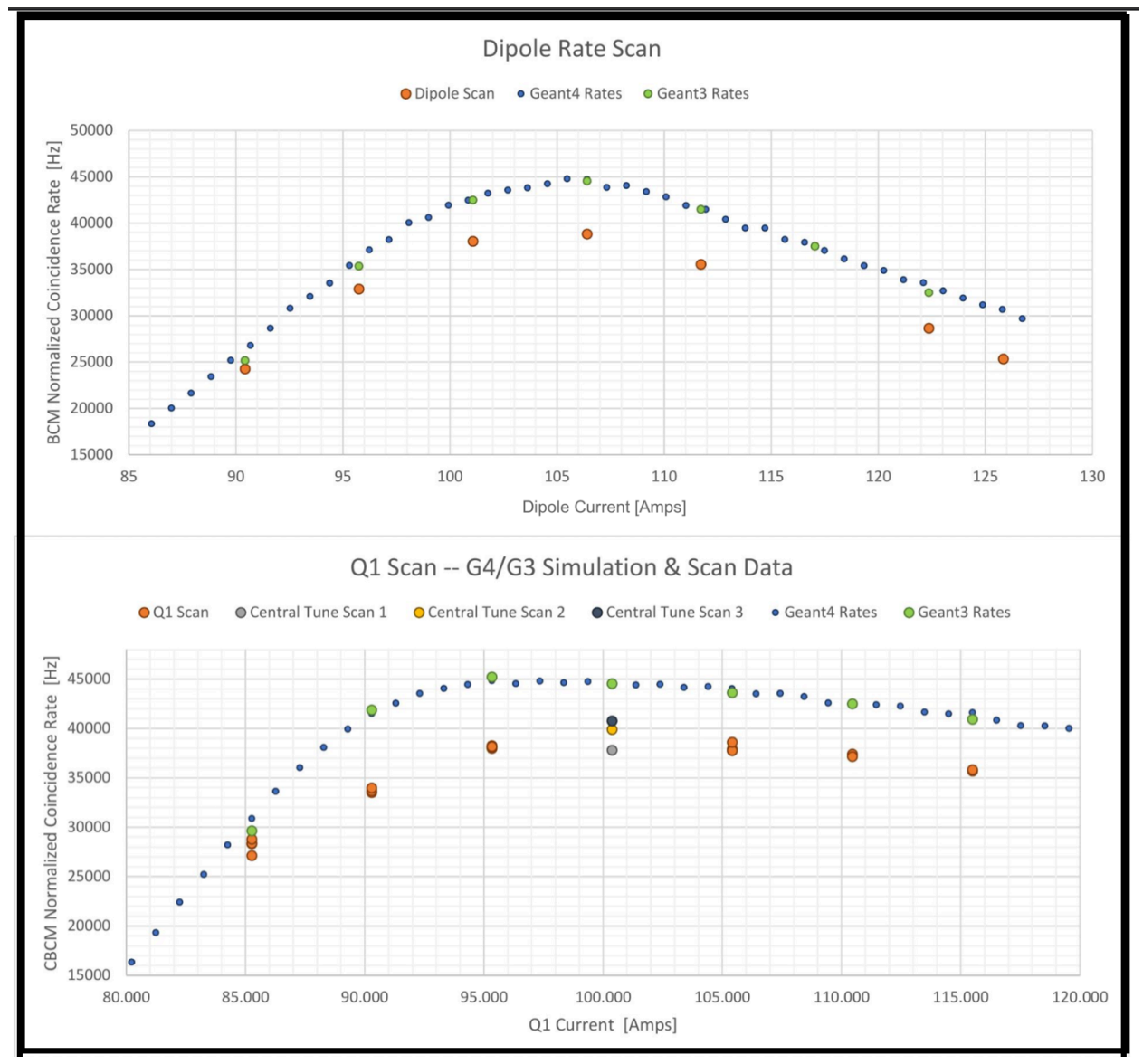

Figure 5.9: 2019 Dipole and Q1 Scan at $2.139 \mathrm{GeV}$. Q3 is off. Plots courtesy of Eric King. 


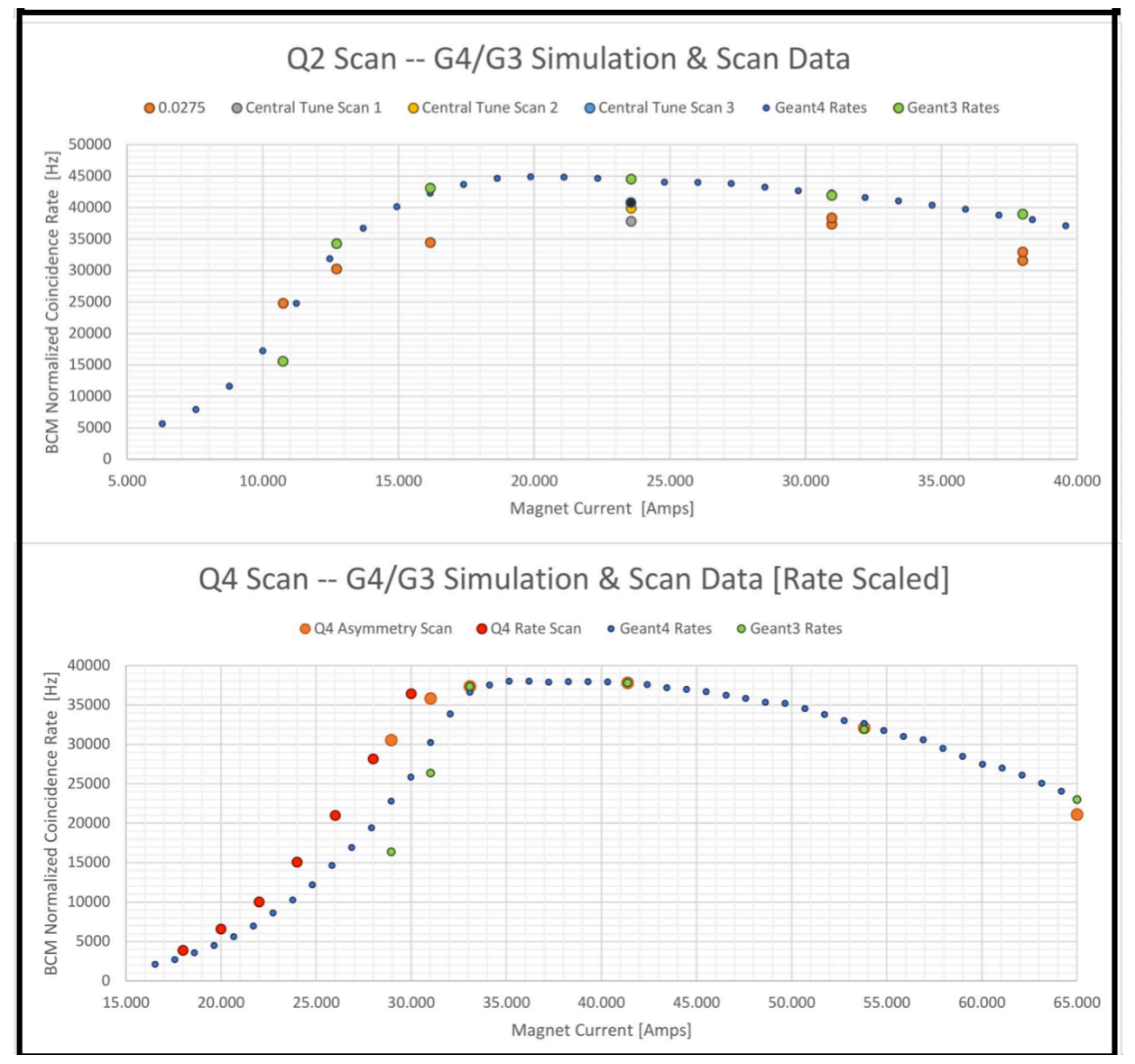

Figure 5.10: 2019 Q2 and Q4 Scan at 2.139 GeV. Q3 is off. Plots courtesy of Eric King. 


\section{CHAPTER 6}

\section{FUTURE PLANNED EXPERIMENTS IN HALL A}

\subsection{PREX-II and CREX}

\subsubsection{Operation at $02.2 \mathrm{GeV}$}

The CREX experiment and the recently completed Møller polarimeter commissioning run both use a beam energy of $2.2 \mathrm{GeV}$. Ths section will focus on the the quadrupole settings that have been developed using Geant3. Optics settings for past Møller measurements at similar energies were used as a starting point. Phase space scans utilizing three quadrupoles were developed as is shown in figure 6.1. Here Q3 is turned off and the other three quadrupoles were varied in 200 gauss steps for all possible combinations of Q1, Q2 and Q4 field strengths. The color scale of the plots shows how many coincidence pairs are detected per 1500 simulated events. Figure 6.1 shows that for any value of Q1, a suitable value of Q2 and Q4 may be chosen as a possible optics solutions at 2.2 GeV. Several of the more interesting solutions were studied and two of them are presented.

First I present the simplest solution, one that only uses Q2 and has the other three quadrupoles turned off. By looking at the plot where Q1 is turned off and reading the value of Q2 when Q4 is off and the coincidences are a maximum we can see Q2 $=2.5 \mathrm{kG}$ is a possible one quad solution. By varying the current in Q2 we can see the effect that is has on the rates, analyzing power, and the Levchuk correction. This simple solution has the advantage of reduced systematic errors due to uncertainities in quadruple alignment, current, and field being limited to only one quadrupole. Additionally, rate scans comparison 


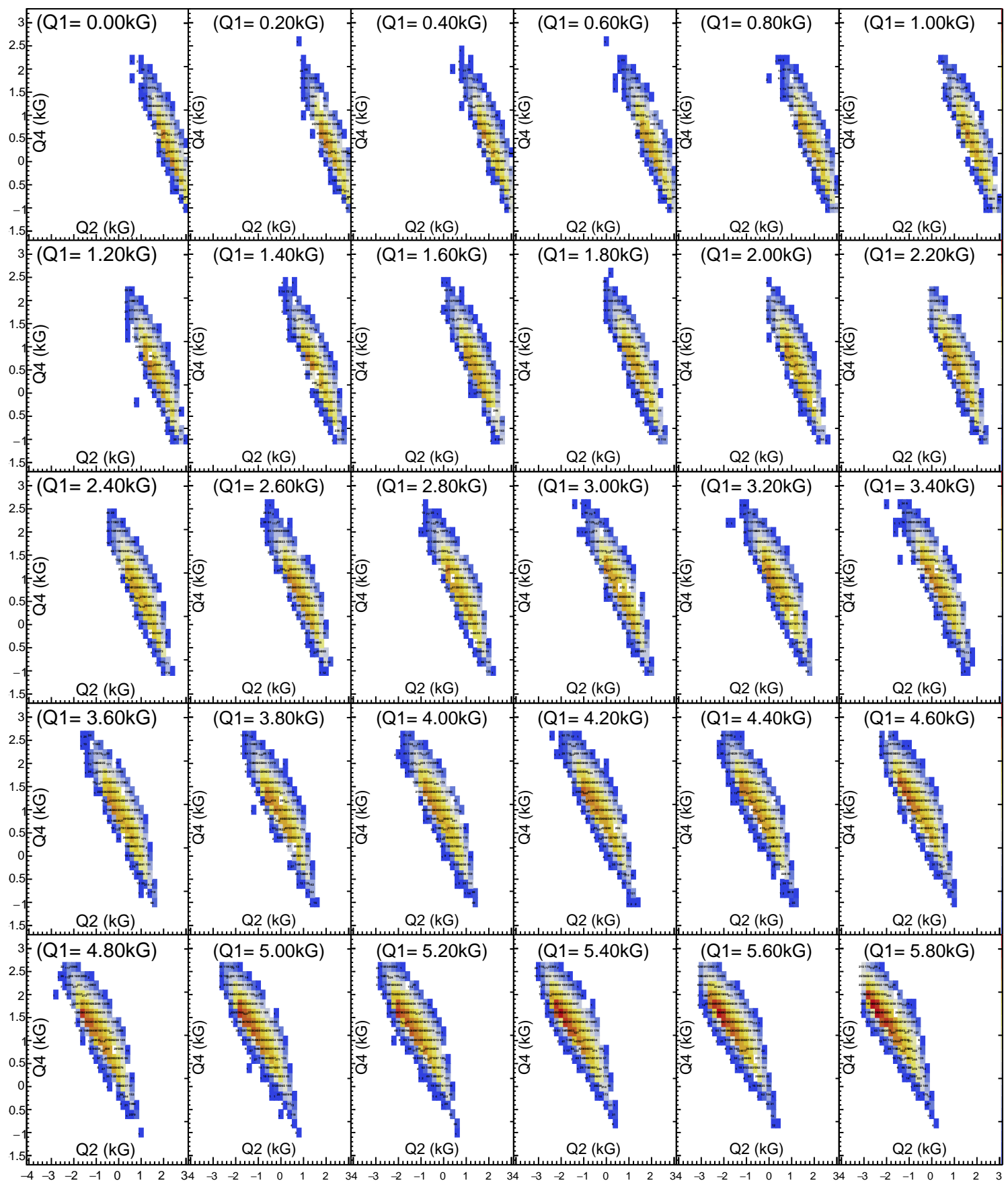

Figure 6.1: Coincidence pairs plotted as Q4 field vs Q2 field for various values of Q1. The redder regions indicate higher rates and possible solutions for a beam energy of $2.2 \mathrm{GeV}$. 
with simulation are less entangled since three of the four quadruple fields are off.

A three quadrupole solution is being proposed for CREX and has already been tested during commissioning. The solution features all three quadrupoles set to focus in the horizontal direction. Q1 does most of the focusing and it set to $2.5 \mathrm{kG}$, while Q2 and Q4 are set to $0.5 \mathrm{kG}$ and $1.0 \mathrm{kG}$ respectively. Q3 is off. This solution was chosen because $\left\langle A_{z z}\right\rangle$ was the least sensitive to changes in the magnet settings. It also features relatively high rates and a small Levchuk correction when compared with other solutions. The effect of varying the field in each of the quadrupoles and dipoles are shown in Figure 6.3 at $2.139 \mathrm{GeV}$ which was the beam energy used during commissioning. The same solution is shown in Figure 6.4 at $2.2 \mathrm{GeV}$, the beam energy for CREX. At $2.2 \mathrm{GeV}$, even with $5 \%$ changes of Q2 and $\mathrm{Q} 4,\left\langle A_{z z}\right\rangle$ is stable within $\sim 0.1 \%$. Q1 is more sensitive however becomes fairly stable as the field is increased in the positive direction.
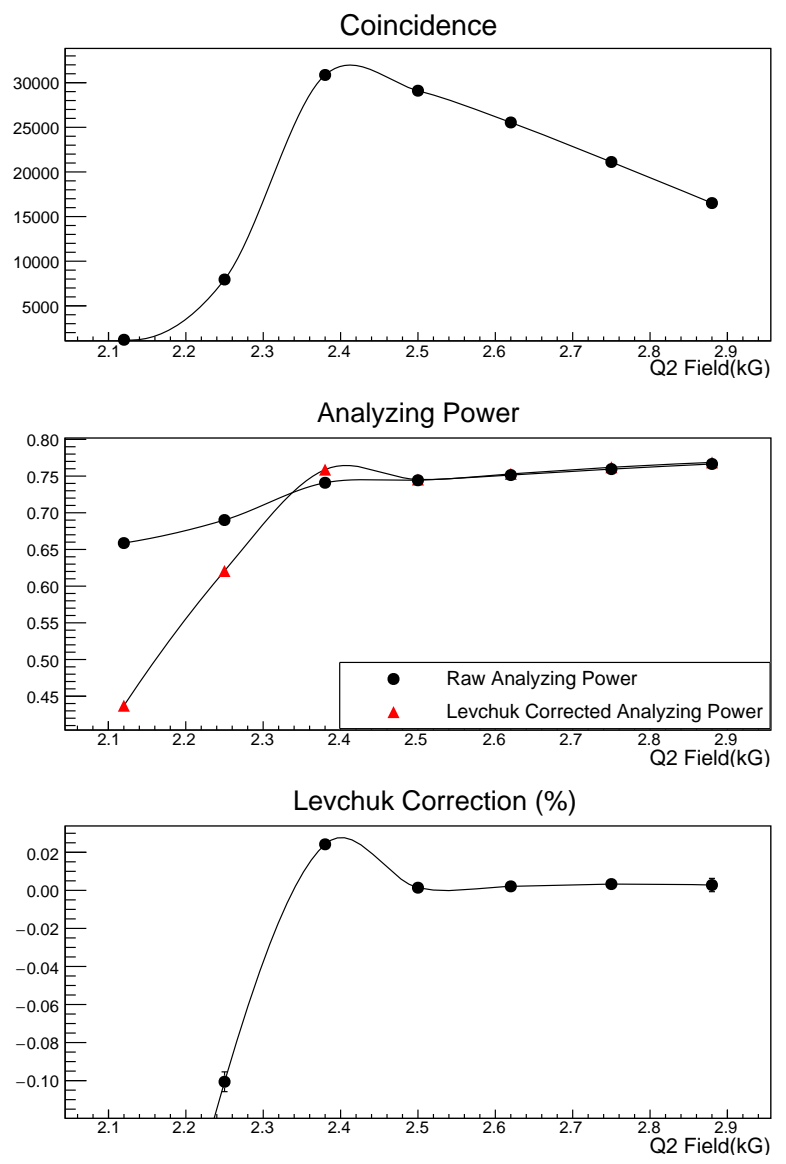

Figure 6.2: Q2 Quad scan at $2.2 \mathrm{GeV}$. This is a one quadrupole solution with the other three quadrupoles turned off. Coincidence (Top), Analyzing Power(Middle), Levchuk Correction(Bottom) 


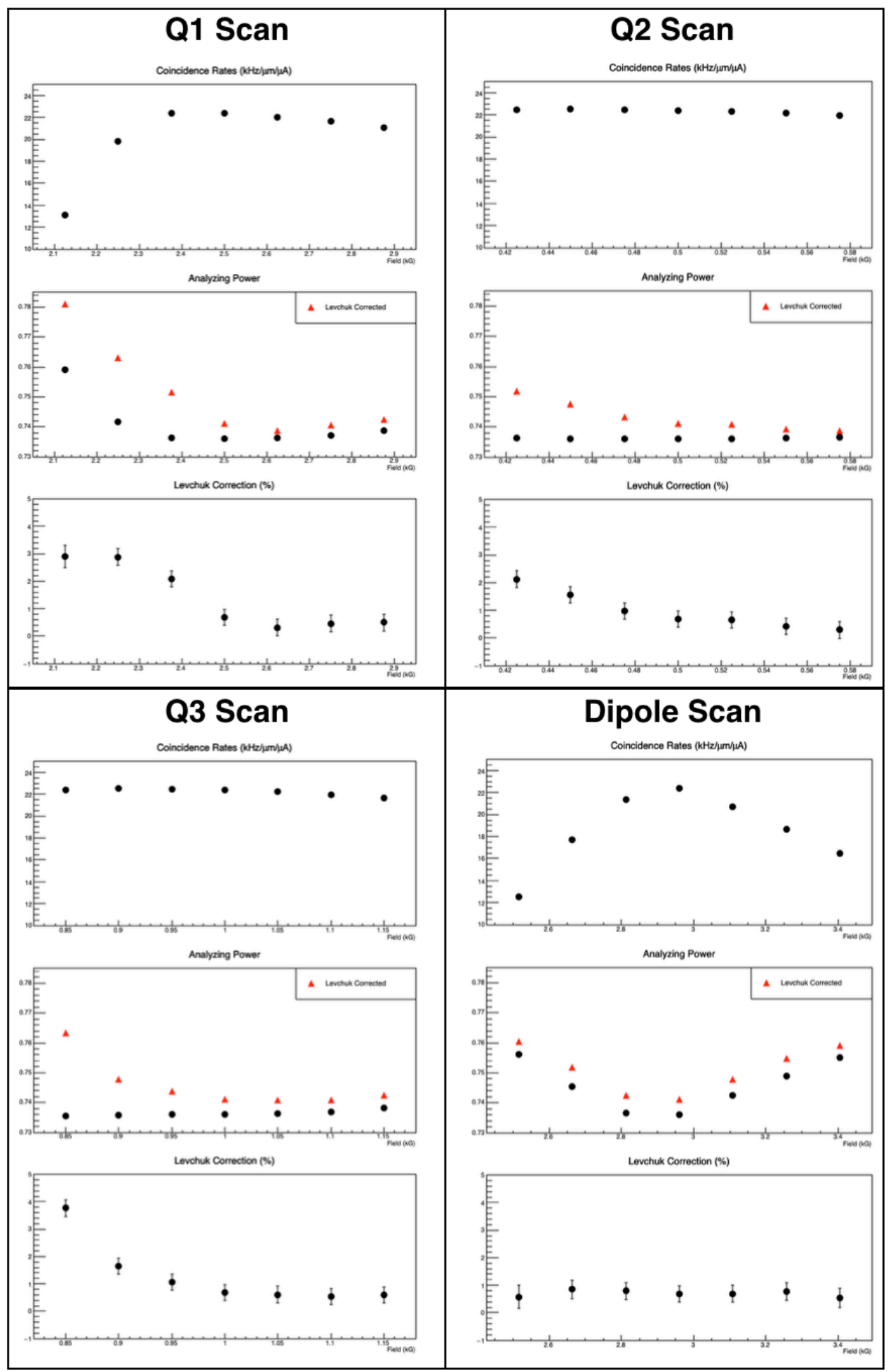

Figure 6.3: Three quadrupole $2.139 \mathrm{GeV}$ solution. This solution also can be used for $2.2 \mathrm{GeV}$ (CREX). Q1=2.5 kG, Q2=0.5 kG, Q3=0 kG and Q4=1.0kG 

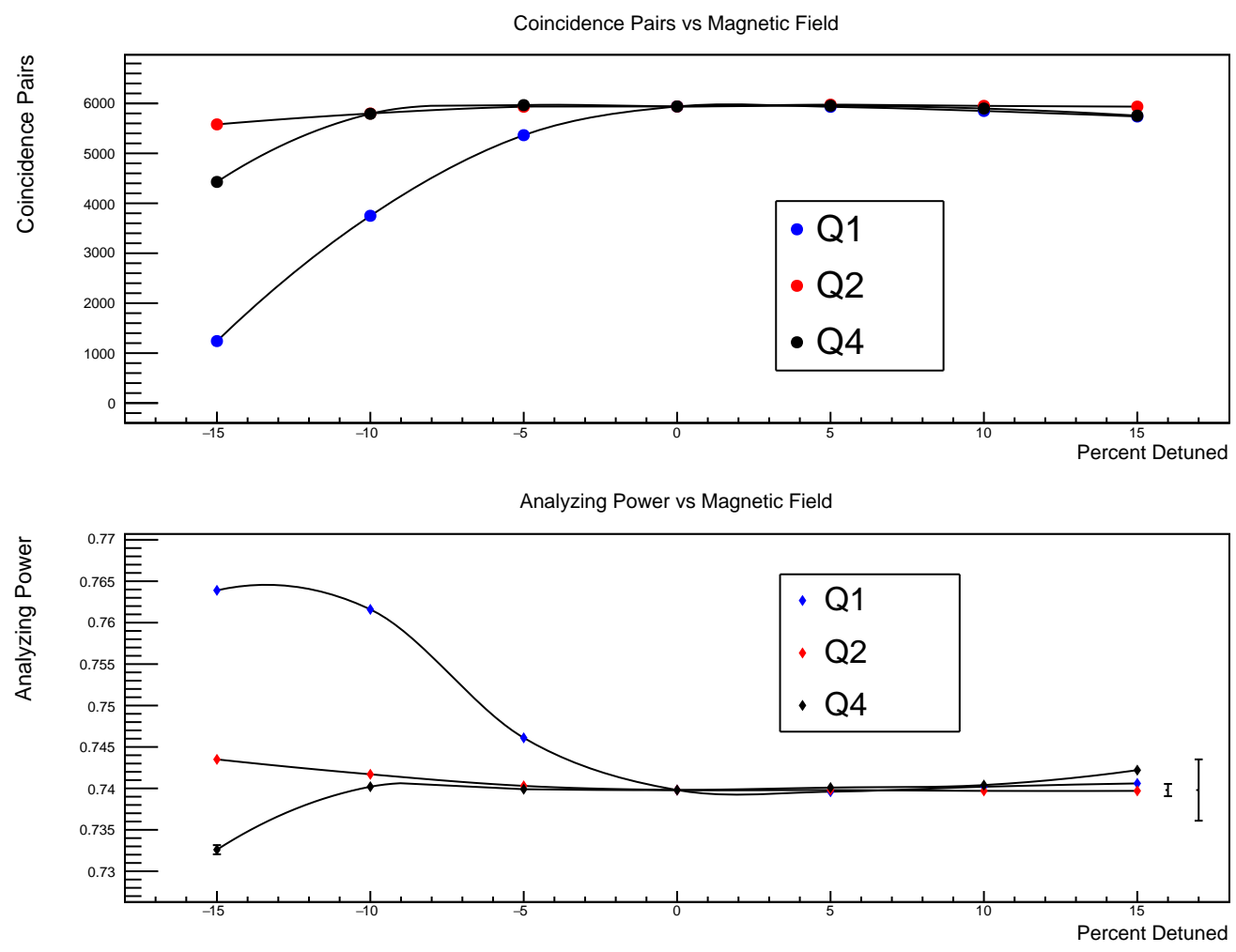

Figure 6.4: Effect of detuning optics components on analyzing power and coincidence rates at $2.2 \mathrm{GeV}$ (CREX). The $\pm 0.5 \%$ and $\pm 0.1 \%$ error bars on right are shown for comparison. 


\subsubsection{Operation at $0.95 \mathrm{GeV}$}
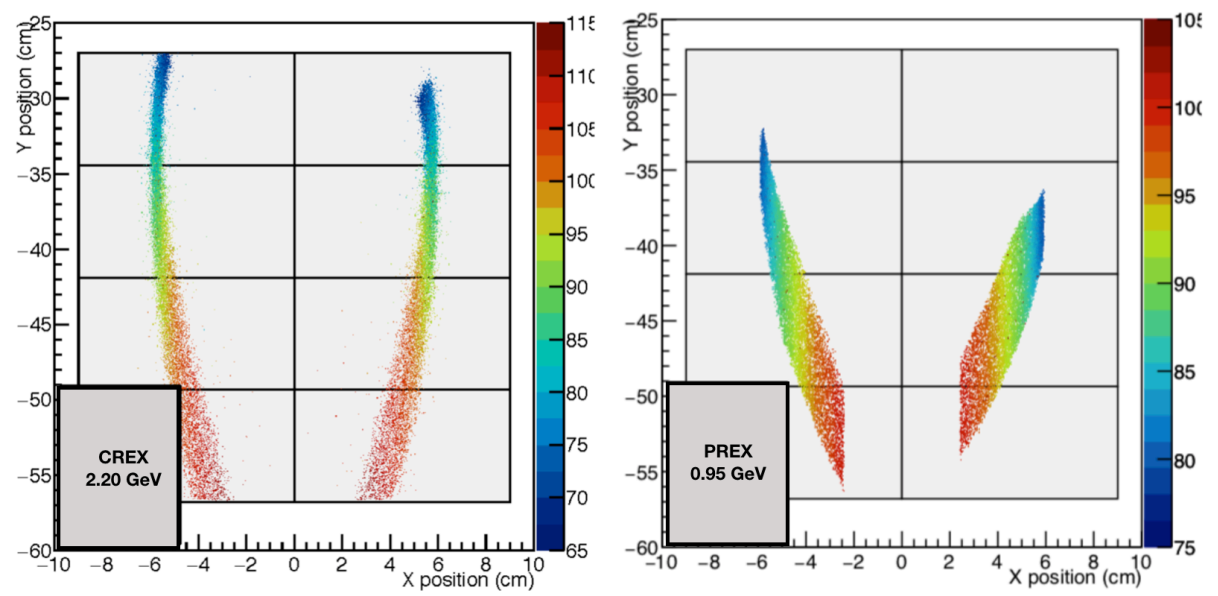

Figure 6.5: Coincidence electrons on detector plane for CREX and PREX spectrometer settings.

The PREX optics solution at $0.95 \mathrm{GeV}$ was found using the same method as described for the CREX solution. The phase space scan is shown in Figure 6.7. The "islands" that make up the solutions are narrower than the "islands" of the $2.2 \mathrm{GeV}$ scans because the electrons have smaller energies and are more sensitive to variations of the fields. A finer phase space scan is shown in Figure 6.8 and was performed in the regions where the coincidence pairs are largest from Q1=2.75 kG to $3.25 \mathrm{kG}$. Here the magnets were varied in 50 Gauss steps. Note that all possible settings are not plotted as is evident from the "holes" that appear in the scans. A solution was chosen with Q1=3.0 kG, Q2=-0.6 kG and Q4=0.6 kG. Again Q3 is turned off. It should also be noted that here Q2 is defocusing in the horizontal direction. The sensitivity of the rates and mean analyzing power as a function of magnet current is shown in Figures 6.9 and 6.10. 5\% changes to the field of Q2 and Q4 result in $<0.1 \%$ changes of $\left\langle A_{z z}\right\rangle$, similar to the CREX solution. Percent level variations of the Q1 field has a much larger effect on $\left\langle A_{z z}\right\rangle$ which is expected since the magnitude of Q1 is five times larger than that of Q2 and Q4. The location of the coincidence electrons are shown in Figure 6.5. The CREX solution has the electrons hitting on a smaller area of the detector. This is favorable because $\left\langle A_{z z}\right\rangle$ is less sensitive to the location of the detector and surrounding geometries and 


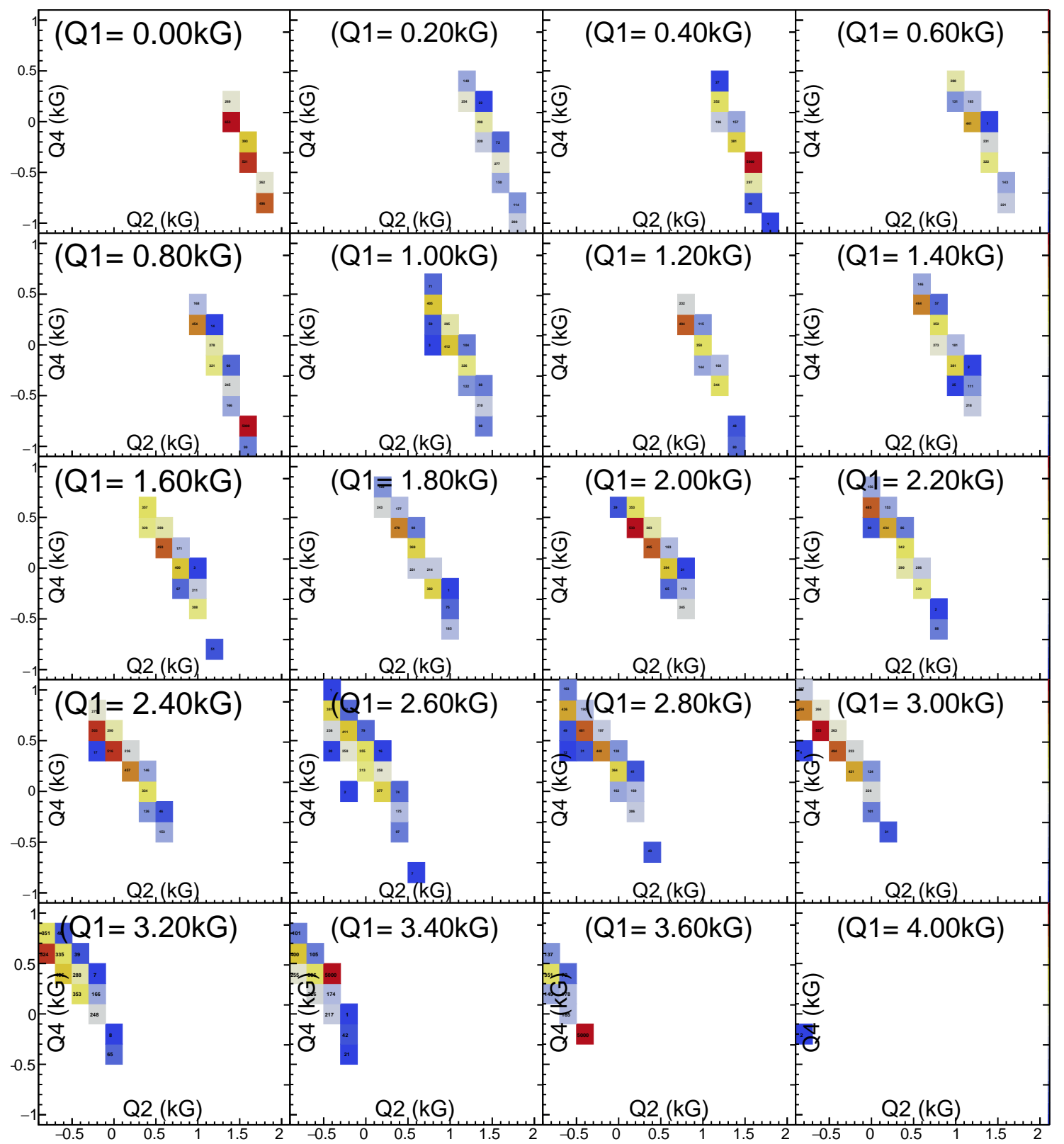

Figure 6.6: Phase Space Scan of Q1, Q2, and Q4 at $0.95 \mathrm{GeV}$ 


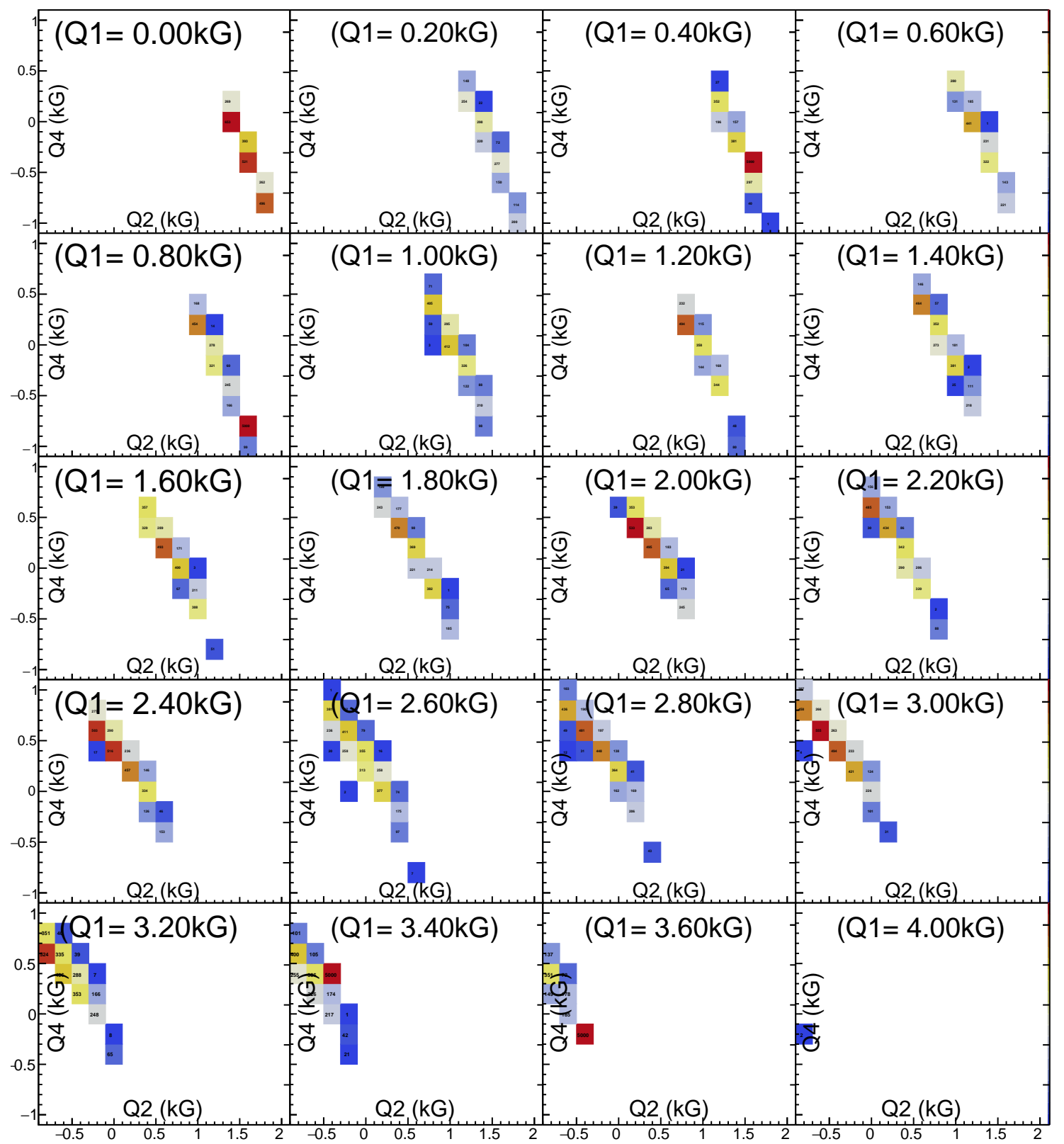

Figure 6.7: Phase Space Scan of Q1, Q2, and Q4 at $0.95 \mathrm{GeV}$ 

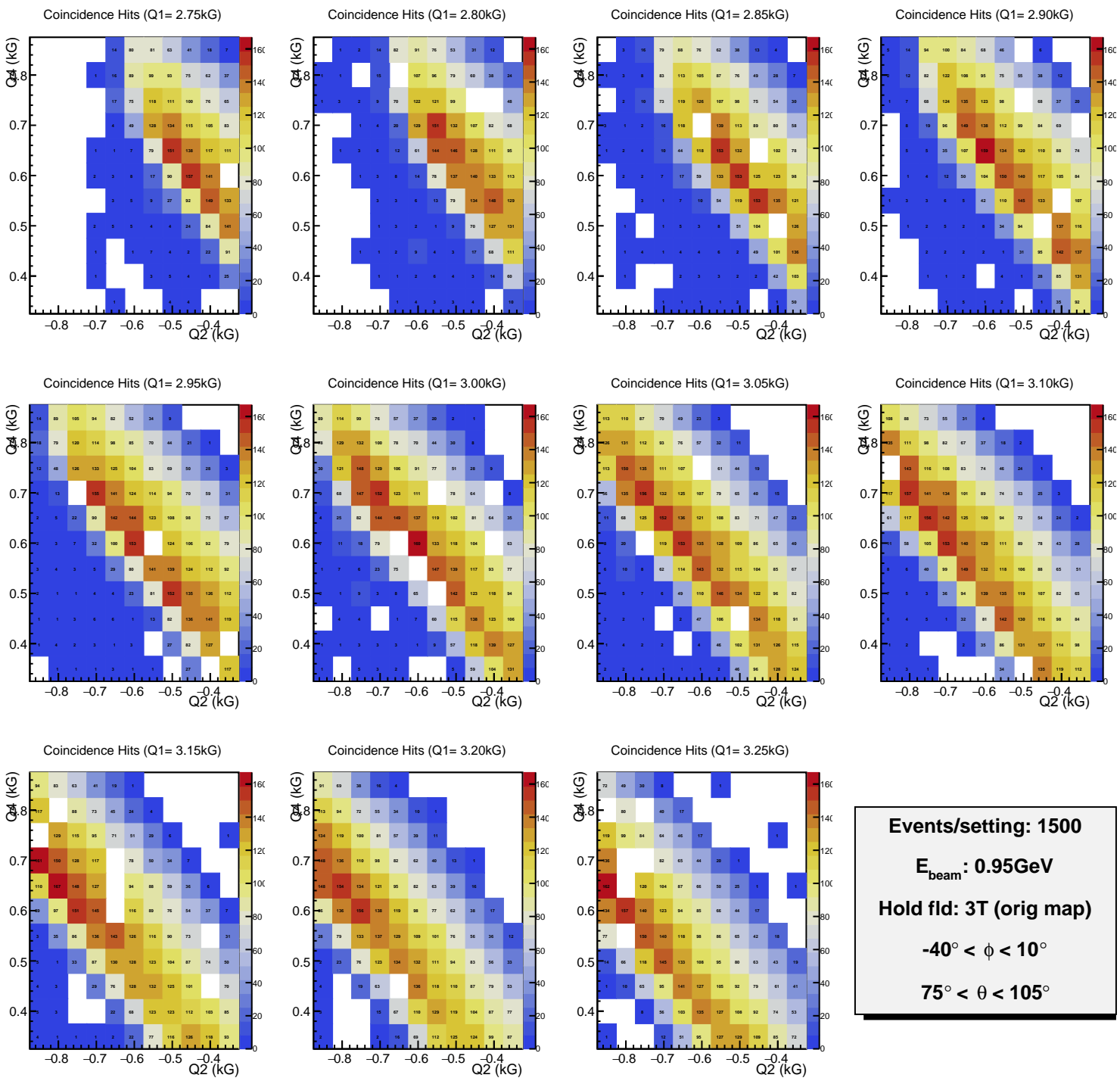

Figure 6.8: Higher resolution phase space scan of Q1, Q2, and Q4 at $0.95 \mathrm{GeV}$ 

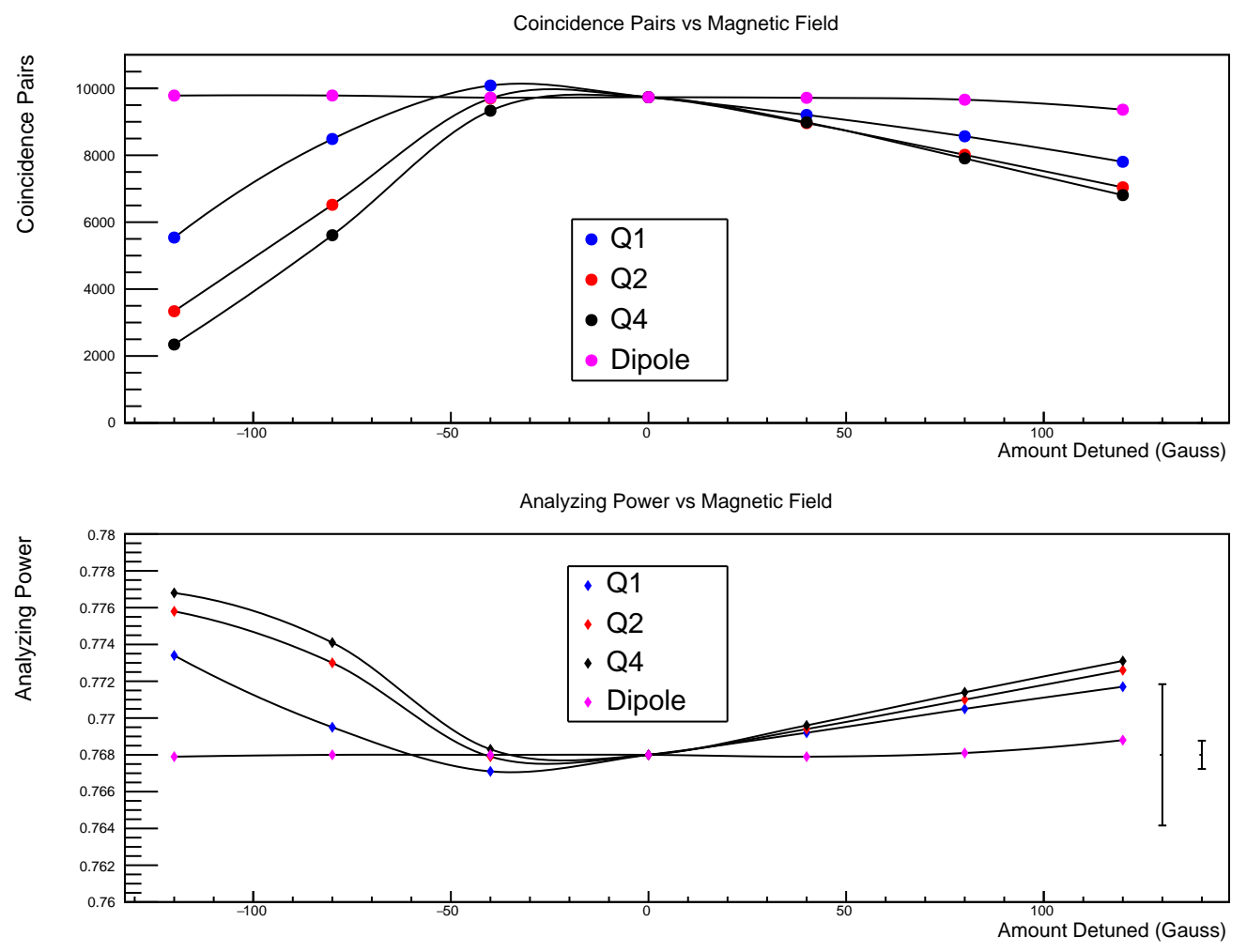

Figure 6.9: Effect of detuning optics components on analyzing power and coincidence rates at $0.95 \mathrm{GeV}$ (PREX). The $\pm 0.5 \%$ and $\pm 0.1 \%$ error bars on right are shown for comparison. 

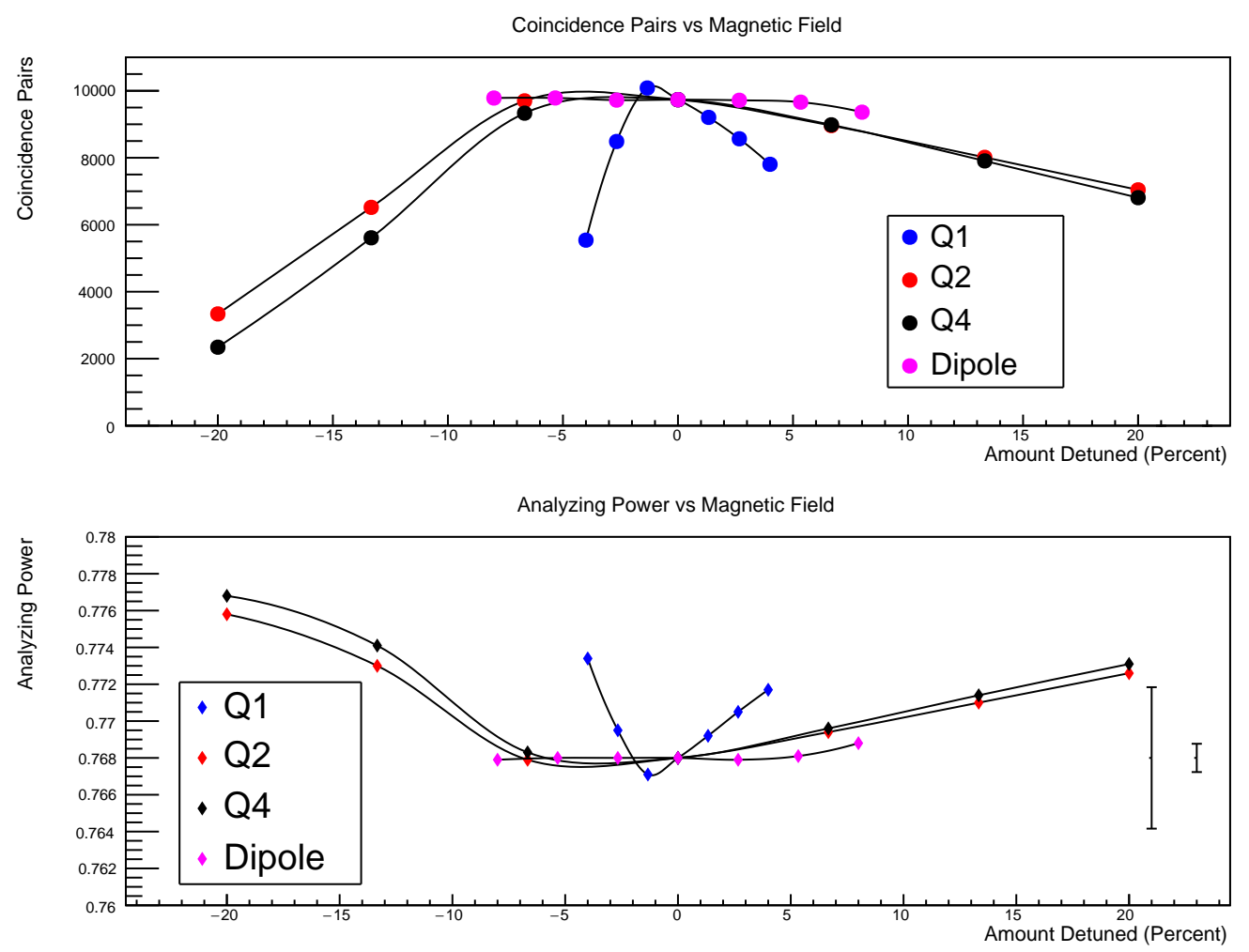

Figure 6.10: Effect of detuning optics components on analyzing power and coincidence rates at $0.95 \mathrm{GeV}$. The $\pm 0.5 \%$ and $\pm 0.1 \%$ error bars on right are shown for comparison. This plot is the same as Figure 6.9 except the $\mathrm{x}$-axis is in percent versus Gauss 


\subsection{Polarimetry for MOLLER and SoLID}

After delivering $0.8 \%$ polarimetry for CREX the MOLLER and SoLID programs will be demanding $0.4 \%$ polarimetry. If the uncertainty in target polarization remains at $0.3 \%$ and statistics can be limited to $0.1 \%$ then the total remaining systematic error must be $<0.25 \%$. The total systematic errors shown in Table 4.2, without the Levchuk correction is $0.36 \%$ but there are several contributions to this may be further reduced with simulation and modeling improvements. Some areas for further reduction of the systematic errors are:

- The Levchuk Effect: Table 4.2 lists the uncertainity of the Levchuk correction to be $33 \%$ which reflects that a detailed study of the correction has not been performed. The accuracy of the correction depends on the probability functions which determine the momentum of the inner shell electrons. Repeating the Levchuk analysis using delta function distributions as in [56] and using atomic momentum distributions as in [57] will quantify the sensitivity of the correction to different models. This was done for the SLAC polarimeter and the different models were found to change the final polarization value by $0.2 \%$, while the correction for that particular polarimeter was $\sim 14 \%$. The Levchuk correction for the Hall A polarimeter is $\sim 10 x$ than the SLAC polarimeter so we can expect the fractional uncertainty of the final result be much smaller as well.

- Magnet Modeling: The quadrupoles and dipole in Geant3 have been implemented as ideal magnets which neglect fringe fields and higher order multipole contributions to the fields. The recent development of the GEANT4 simulation has included implementation of field maps for the quadrupoles from TOSCA models. Improving the magnetic modeling in the simulation should more accurately predict the acceptance of the detector thus improving the agreement between simulation and data.

- Modeling in GEANT4: The modeling of the apparatus is Geant3/GEANT4 has been carefully checked and studied but there are still some areas of uncertainty. They are mainly in the dipole and collimator regions because detailed drawings of the assembly to not exist. Opening the flange at the dipole entrance would allow for the exact positions of the various elements to be measured and refined in the simulation. Measurements should include the collimator dimensions, the width of the dipole aperature, the z-location of the solid box which divides the dipole apertures and the various screws and other parts which make up the collimator assembly (See 
Figure 3.14). There has also been some conflicting information regarding the inner diameter of the beam pipe which should be measured as well. Detailed drawings for the main detector also do not exist therefore it should also be measured and documented. This is particularly important because survey measures the relative position to the back of the detector and without knowing the length in $\mathrm{z}$ of the detector package makes it difficult to know with high precision the actual position of the front of the detector (where the electrons are hitting).

- Radiative Corrections: GEANT4 includes radiative corrections which were not implemented in Geant3. The implementation and modeling of the correction in the new simulation needs to be documented and systematic studies should be performed.

- Multiple Optics Configurations: Since multiple optics configurations exist for each beam energy, each with different analyzing powers, Levchuk corrections, and radiative corrections, then using several optics solutions can provide a cross-check of the simulation.

- Dedicated Beam Time for Systematic Studies: Dedicated beam time for Møller polarimetry should be requested to perform quadrupole scans and target studies. Quadrupole scans will allow for rate comparisons between simulation and data. High precision scans will also allow for validation of the simulation since each point has different analyzing powers and corrections. Target angle scans should be performed, provided that there is $<0.1 \%$ angular resolution, in order to verify alignment. Target saturation tests should be preformed by varying the target holding field and measuring the asymmetry in order to verify the target is saturated. Additionally, target position scans may be performed by varying the location of the beam on target to ensure that the measured asymmetry does not change due to wrinkles or other imperfections in the foil. In order to provide $0.4 \%$ precision a systematic study program will be necessary and beam time will need to be allocated.

- Target Polarization: Any future studies that would increase the precision of which we know the target magnetization would be an exciting development for precision polarimetry. Presently XMCD studies and are being discussed but achieving $<0.3 \%$ is a challenge and no clear means of improvement is presently known.

Figure 6.11 shows the detector coincidence acceptance in maroon for a beam energy of $11 \mathrm{GeV}$ (MOLLER) as well as for CREX and PREX energies for comparison. At $11 \mathrm{GeV}$, 
all four quadrupoles are used, with the first two being set to defocus in the horizontal direction since the lab scattering angle is smaller at higher beam energies. The acceptance of the detector is defined by the higher energy electron from the Møller pair on the face of the inner dipole box. Figure 6.12 shows electron pairs generated in $\theta_{C M}$ from $100^{\circ}$ to $115^{\circ}$ and the complimentary electron from $65^{\circ}$ to $80^{\circ}$. The fact that the acceptance is defined solely at the dipole face makes the acceptance less sensitivity to ill-defined geometries further downstream.

The Levchuk correction at $11 \mathrm{GeV}$ is $1.15 \% \pm 0.10 \%$ (stat.). This correction was produced by using the $11 \mathrm{GeV}$ tune that was used on March 31st, 2016 for the DVCS experiment. The size of the correction is approximately twice the size of the CREX/PREX and this can be explained by the $\theta_{C M}$ acceptance being half that of CREX/PREX (see Figure 6.11 since Levchuk has shown the size of the correction is related $\Delta \theta / \theta$ [56].
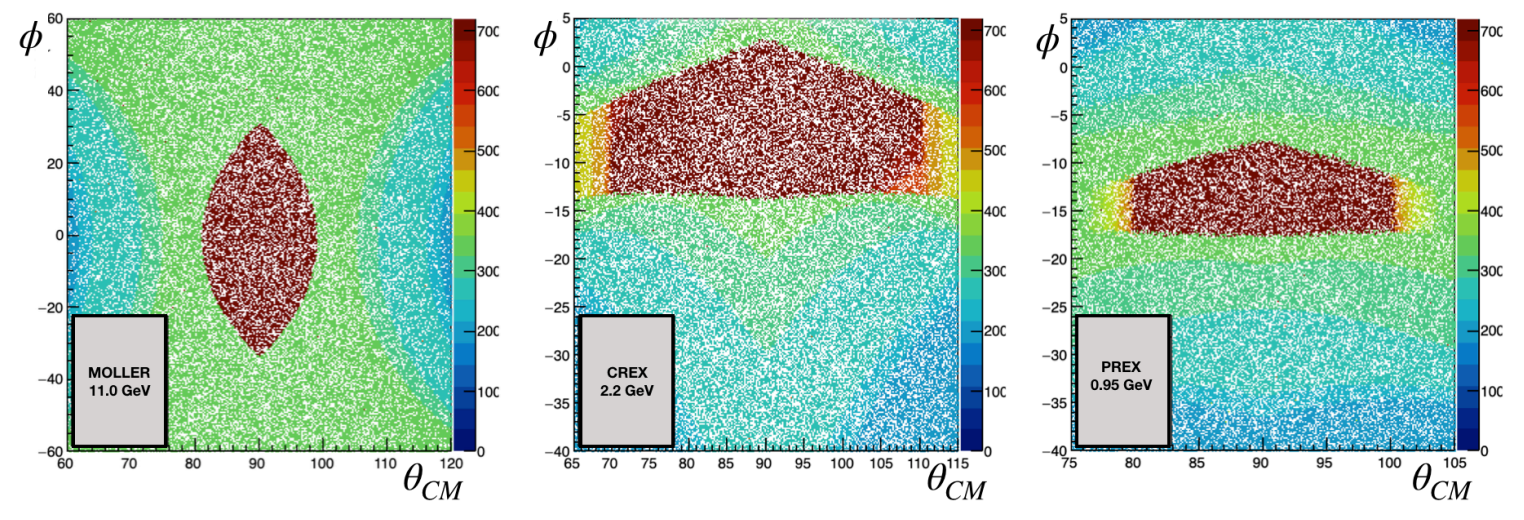

Figure 6.11: The detector acceptance (in maroon) for MOLLER, CREX, and PREX beam energies. The color scale represents the z-location of the electron that died furthest upstream. For a beam energy of $11 \mathrm{GeV}$ (MOLLER) the acceptance is defined entirely on the dipole face. This can be seen by the green surrounding the maroon. At CREX and PREX energies the acceptance is also defined inside the dipole box (yellow/orange) and on the detector shielding box (red).

In conclusion with further systematic studies, in conjunction with improvements upon the simulation, $0.4 \%$ polarimetry will be possible in order to meet the demands of future experiments. Table 4.4 indicates the total systematic error calculated thus far is $0.47 \%$ and ways of reducing this errors have been outlined above. However this table should be reproduced for other beam energies, namely $11 \mathrm{GeV}$ for the MOLLER experiment. At 


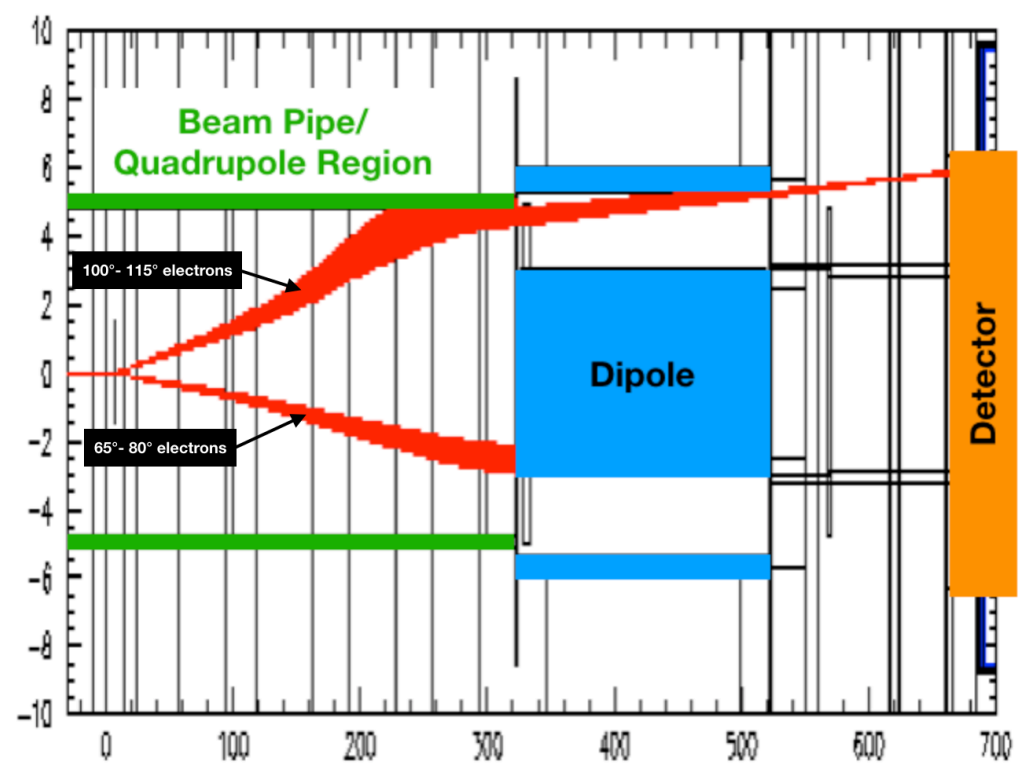

Figure 6.12: Electron pairs generated in $\theta_{C M}$ from $100^{\circ}$ to $115^{\circ}$ and the complimentary electron from $65^{\circ}$ to $80^{\circ}$. The high energy electrons define the coincidence acceptance at the dipole face.

the present time it appears that there is no real show stoppers for reaching the desired precision and with careful planning and systematic studies using simulation, the Hall A Møller Polarimeter will be able to deliver $<0.5 \%$ polarimetry. 


\section{BIBLIOGRAPHY}

[1] CF Perdrisat, V Punjabi, and M Vanderhaeghen. Nucleon electromagnetic form factors. Progress in Particle and Nuclear Physics, 59(2):694-764, 2007.

[2] J Arrington, PG Blunden, and W Melnitchouk. Review of two-photon exchange in electron scattering. Progress in Particle and Nuclear Physics, 66(4):782-833, 2011.

[3] C Prescott. Weak-electromagnetic interference in polarized electron-deuteron scattering. Technical report, P00008322, 1992.

[4] J. KESSLER. Electron spin polarization by low-energy scattering from unpolarized targets. Rev. Mod. Phys., 41:3-25, Jan 1969.

[5] M Steigerwald. Mev mott polarimetry at jefferson lab. In AIP conference proceedings, volume 570, pages 935-942. AIP, 2001.

[6] Kurt Aulenbacher, Eugene Chudakov, David Gaskell, Joseph Grames, and Kent D Paschke. Precision electron beam polarimetry for next generation nuclear physics experiments. International Journal of Modern Physics E, 27(07):1830004, 2018.

[7] Donald Jones. Measuring the weak charge of the proton via elastic electron-proton scattering. arXiv preprint arXiv:1601.07172, 2016.

[8] Megan Friend, D Parno, F Benmokhtar, A Camsonne, MM Dalton, GB Franklin, V Mamyan, R Michaels, S Nanda, V Nelyubin, et al. Upgraded photon calorimeter with integrating readout for the hall a compton polarimeter at jefferson lab. Nuclear Instruments and Methods in Physics Research Section A: Accelerators, Spectrometers, Detectors and Associated Equipment, 676:96-105, 2012.

[9] O Glamazdin. Moeller (iron foils) existing techniques. Nuovo Cimento-C, 35(4):176, 2012. 
[10] A. V. Glamazdin et al. Electron beam Moller polarimeter at JLab Hall A. Fizika, B8:91-95, 1999.

[11] Quadrupole magnet. https://en.wikipedia.org/wiki/Quadrupole_ magnet. Accessed: 2019-02-26.

[12] Quadrupoles in simulation. https://hallaweb.jlab.org/equipment/ moller/magnets/quad_summary_simul.html. Accessed: 2019-02-28.

[13] J Crangle and GM Goodman. The magnetization of pure iron and nickel. Proc. $R$. Soc. Lond. A, 321(1547):477-491, 1971.

[14] LV De Bever, J Jourdan, M Loppacher, S Robinson, I Sick, and J Zhao. A target for precise møller polarimetry. Nuclear Instruments and Methods in Physics Research Section A: Accelerators, Spectrometers, Detectors and Associated Equipment, 400(23):379-386, 1997.

[15] R Pauthenet. Spin-waves in nickel, iron, and yttrium-iron garnet. Journal of Applied Physics, 53(3):2029-2031, 1982.

[16] Target polarization tech note. https://hallaweb.jlab.org/dvcslog/ moller/17. Accessed: 2019-03-10.

[17] J Benesch, P Brindza, RD Carlini, JP Chen, E Chudakov, S Covrig, MM Dalton, A Deur, D Gaskell, A Gavalya, et al. The moller experiment: An ultra-precise measurement of the weak mixing angle using møller scattering. arXiv preprint arXiv:1411.4088, 2014.

[18] CT Chen, YU Idzerda, H-J Lin, NV Smith, G Meigs, E Chaban, GH Ho, E Pellegrin, and $\mathrm{F}$ Sette. Experimental confirmation of the X-ray magnetic circular dichroism sum rules for iron and cobalt. Physical review letters, 75(1):152, 1995.

[19] W. B. Atwood, R. L. A. Cottrell, H. DeStaebler, R. Miller, H. Pessard, C. Y. Prescott, L. S. Rochester, R. E. Taylor, M. J. Alguard, J. Clendenin, P. S. Cooper, R. D. Ehrlich, V. W. Hughes, M. S. Lubell, G. Baum, K. P. Schüler, and K. Lübelsmeyer. Search for parity violation in deep-inelastic scattering of polarized electrons by unpolarized deuterons. Phys. Rev. D, 18:2223-2226, Oct 1978. 
[20] EJ Beise, ML Pitt, and DT Spayde. The sample experiment and weak nucleon structure. Progress in Particle and Nuclear Physics, 54(1):289-350, 2005.

[21] MK Jones, Jefferson Lab Hall A Collaboration, et al. The ratio of proton's electric to magnetic form factors measured by polarization transfer. arXiv preprint nuclex/9910005, 1999.

[22] A Airapetian, N Akopov, Z Akopov, A Andrus, EC Aschenauer, W Augustyniak, R Avakian, A Avetissian, E Avetissian, S Belostotski, et al. Precise determination of the spin structure function $\mathrm{g} 1$ of the proton, deuteron, and neutron. Physical Review D, 75(1):012007, 2007.

[23] K Abe, T Akagi, BD Anderson, PL Anthony, RG Arnold, T Averett, HR Band, CM Berisso, P Bogorad, H Borel, et al. Precision determination of the neutron spin structure function g 1 n. Physical Review Letters, 79(1):26, 1997.

[24] FE Maas, P Achenbach, K Aulenbacher, S Baunack, L Capozza, J Diefenbach, K Grimm, Y Imai, T Hammel, D Von Harrach, et al. Measurement of strange-quark contributions to the nucleon's form factors at q $2=0.230(\mathrm{~g} \mathrm{e} \mathrm{v/c}) 2$. Physical review letters, 93(2):022002, 2004.

[25] DS Armstrong, J Arvieux, Razmik Asaturyan, Todd Averett, SL Bailey, Guillaume Batigne, DH Beck, EJ Beise, Jay Benesch, Louis Bimbot, et al. Strange-quark contributions to parity-violating asymmetries in the forward G0 electron-proton scattering experiment. Physical review letters, 95(9):092001, 2005.

[26] Darko Androić, DS Armstrong, J Arvieux, SL Bailey, DH Beck, EJ Beise, J Benesch, F Benmokhtar, L Bimbot, J Birchall, et al. Strange quark contributions to parity-violating asymmetries in the backward angle g0 electron scattering experiment. Physical review letters, 104(1):012001, 2010.

[27] D Androic, DS Armstrong, A Asaturyan, T Averett, J Balewski, J Beaufait, RS Beminiwattha, J Benesch, F Benmokhtar, J Birchall, et al. First determination of the weak charge of the proton. Physical review letters, 111(14):141803, 2013.

[28] Koya Abe, Kenji Abe, T Abe, I Adam, H Akimoto, D Aston, KG Baird, C Baltay, HR Band, TL Barklow, et al. Improved direct measurement of leptonic coupling asymmetries with polarized z bosons. Physical review letters, 86(7):1162, 2001. 
[29] D Androic, DS Armstrong, A Asaturyan, T Averett, J Balewski, J Beaufait, RS Beminiwattha, J Benesch, F Benmokhtar, J Birchall, et al. First determination of the weak charge of the proton. Physical review letters, 111(14):141803, 2013.

[30] High flux parity experiments at jlab: Prex, crex and moller. http: //www.ectstar.eu/sites/www.ectstar.eu/files/talks/03_ mcnulty_pvect2016.pdf. Accessed: 2019-02-12.

[31] Robert Michaels. Electroweak measurements of neutron densities in prex and crex at jlab, usa. arXiv preprint arXiv:1510.04592, 2015.

[32] Juliette Mammei et al. The moller experiment. arXiv preprint arXiv:1208.1260, 2012.

[33] Precision measurement of parity-violation in deep inelastic scattering over a broad kinematic range. https://hallaweb.jlab.org/collab/PAC/PAC34/ PR-09-012-pvdis.pdf. Accessed: 2019-03-10.

[34] LS Cardman. Polarized electron sources: The next generation. In AIP Conference Proceedings, volume 243, pages 1012-1019. AIP, 1992.

[35] LS Cardman. Polarized electron sources for the 1990's. Nuclear Physics A, 546(12):317-336, 1992.

[36] CK Sinclair, PA Adderley, BM Dunham, JC Hansknecht, P Hartmann, M Poelker, JS Price, PM Rutt, WJ Schneider, and M Steigerwald. Development of a high average current polarized electron source with long cathode operational lifetime. Physical Review Special Topics-Accelerators and Beams, 10(2):023501, 2007.

[37] V Tioukine, K Aulenbacher, and E Riehn. A mott polarimeter operating at mev electron beam energies. Review of Scientific Instruments, 82(3):033303, 2011.

[38] James L McCarter, Marcy L Stutzman, Kenneth W Trantham, Timothy G Anderson, April M Cook, and Timothy J Gay. A low-voltage retarding-field mott polarimeter for photocathode characterization. Nuclear Instruments and Methods in Physics Research Section A: Accelerators, Spectrometers, Detectors and Associated Equipment, 618(13):30-36, 2010.

[39] Douglas W Higinbotham. Electron spin precession at cebaf. In AIP Conference Proceedings, volume 1149, pages 751-754. AIP, 2009. 
[40] JA Magee, A Narayan, D Jones, R Beminiwattha, JC Cornejo, MM Dalton, W Deconinck, D Dutta, D Gaskell, JW Martin, et al. A novel comparison of møller and compton electron-beam polarimeters. Physics Letters B, 766:339-344, 2017.

[41] M. Hauger et al. A High precision polarimeter. Nucl. Instrum. Meth., A462:382-392, 2001.

[42] HR Band, G Mitchell, R Prepost, and T Wright. A møller polarimeter for high energy electron beams. Nuclear Instruments and Methods in Physics Research Section A: Accelerators, Spectrometers, Detectors and Associated Equipment, 400(1):24-33, 1997.

[43] GW Ford and CJ Mullin. Scattering of polarized dirac particles on electrons. Physical Review, 108(2):477, 1957.

[44] Adam M Bincer. Scattering of longitudinally polarized fermions. Physical Review, 107(5):1434, 1957.

[45] P Stehle. Calculation of electron-electron scattering. Physical Review, 110(6):1458, 1958.

[46] A Raczka and R Raczka. Moller scattering of arbitrarily polarized electrons. Physical Review (US) Superseded in part by Phys. Rev. A, Phys. Rev. B: Solid State, Phys. Rev. C, and Phys. Rev. D, 110, 1958.

[47] B Wagner, HG Andresen, KH Steffens, W Hartmann, W Heil, and E Reichert. A møller polarimeter for $\mathrm{cw}$ and pulsed intermediate energy electron beams. Nuclear Instruments and Methods in Physics Research Section A: Accelerators, Spectrometers, Detectors and Associated Equipment, 294(3):541-548, 1990.

[48] J Arrington, EJ Beise, BW Filippone, TG O’Neill, WR Dodge, GW Dodson, KA Dow, and JD Zumbro. A variable energy møller polarimeter at the mit-bates linear accelerator center. Nuclear Instruments and Methods in Physics Research Section A: Accelerators, Spectrometers, Detectors and Associated Equipment, 311(1-2):39-48, 1992.

[49] KB Beard, R Madey, W-M Zhang, DM Manley, BD Anderson, AR Baldwin, JM Cameron, CC Chang, GW Dodson, K Dow, et al. Measurement of the polarization of a pulsed electron beam with a møller polarimeter in the coincidence mode. 
Nuclear Instruments and Methods in Physics Research Section A: Accelerators, Spectrometers, Detectors and Associated Equipment, 361(1-2):46-52, 1995.

[50] HR Band, G Mitchell, R Prepost, and T Wright. A møller polarimeter for high energy electron beams. Nuclear Instruments and Methods in Physics Research Section A: Accelerators, Spectrometers, Detectors and Associated Equipment, 400(1):24-33, 1997.

[51] P Steiner, A Feltham, I Sick, M Zeier, and B Zihlmann. A high-rate coincidence møller polarimeter. Nuclear Instruments and Methods in Physics Research Section A: Accelerators, Spectrometers, Detectors and Associated Equipment, 419(1):105-120, 1998.

[52] Peter Bartsch. Aufbau eines møller-polarimeters für die drei-spektrometer-anlage und messung der helizitätsasymmetrie in der reaktion $\mathrm{p}(\mathrm{e} \rightarrow, \mathrm{e}$ ' $\mathrm{p}) \pi 0$ im bereich der $\delta$-resonanz. 2001.

[53] T Speckner, G Anton, W von Drachenfels, F Frommberger, K Helbing, M Hoffmann, B Kiel, T Michel, J Naumann, and G Zeitler. The gdh-møller-polarimeter at elsa. Nuclear Instruments and Methods in Physics Research Section A: Accelerators, Spectrometers, Detectors and Associated Equipment, 519(3):518-531, 2004.

[54] Bernhard A Mecking, G Adams, S Ahmad, E Anciant, M Anghinolfi, B Asavapibhop, G Asryan, G Audit, T Auger, H Avakian, et al. The cebaf large acceptance spectrometer (clas). Nuclear Instruments and Methods in Physics Research Section A: Accelerators, Spectrometers, Detectors and Associated Equipment, 503(3):513$553,2003$.

[55] D Gaskell, DG Meekins, and C Yan. New methods for precision møller polarimetry. In Proceedings of The 3rd Workshop From Parity Violation to Hadronic Structure and more..., pages 261-264. Springer, 2007.

[56] LG Levchuk. The intra-atomic motion of bound electrons as a possible source of the systematic error in electron beam polarization measurements by means of a möller polarimeter. Nuclear Instruments and Methods in Physics Research Section A: Accelerators, Spectrometers, Detectors and Associated Equipment, 345(3):496-499, 1994. 
[57] M Swartz, HR Band, FJ Decker, P Emma, MJ Fero, R Frey, R King, A Lath, T Limberg, R Prepost, et al. Observation of target electron momentum effects in single-arm mœller polarimetry. Nuclear Instruments and Methods in Physics Research Section A: Accelerators, Spectrometers, Detectors and Associated Equipment, 363(3):526-537, 1995.

[58] David J DeTroye and Ronald J Chase. The calculation and measurement of helmholtz coil fields. Technical report, Army Research Lab Adelphi MD, 1994.

[59] Bernard Dennis Cullity and Chad D Graham. Introduction to magnetic materials. John Wiley \& Sons, 2011.

[60] Diancheng Wang. Measurement of the parity-violating asymmetry in deep inelastic scattering at jlab 6 gev. Technical report, Thomas Jefferson National Accelerator Facility, Newport News, VA (United States), 2013.

[61] The study of dead time of the moller daqs. https://hallaweb.jlab.org/ equipment/moller/wiki/daq/dead_time.html. Accessed: 2019-03-10.

[62] H Danan, A Herr, and AJP Meyer. New determinations of the saturation magnetization of nickel and iron. Journal of Applied Physics, 39(2):669-670, 1968.

[63] CD Graham Jr. Iron and nickel as magnetization standards. Journal of Applied Physics, 53(3):2032-2034, 1982.

[64] RA Reck and DL Fry. Orbital and spin magnetization in fe-co, fe-ni, and ni-co. Physical Review, 184(2):492, 1969.

[65] Willie Sucksmith. The measurement of magnetic saturation intensities at different temperatures. Proceedings of the Royal Society of London. Series A. Mathematical and Physical Sciences, 170(943):551-560, 1939.

[66] Felix Bloch. Zur theorie des ferromagnetismus. Zeitschrift für Physik, 61(3-4):206$219,1930$.

[67] SD Hanham, AS Arrott, and B Heinrich. Dependence of anisotropy field on magnetization in iron. Journal of Applied Physics, 52(3):1941-1943, 1981.

[68] GG Scott and HW Sturner. Magnetomechanical ratios for fe-co alloys. Physical Review, 184(2):490, 1969. 
[69] J Stöhr. X-ray magnetic circular dichroism spectroscopy of transition metal thin films. Journal of Electron Spectroscopy and Related Phenomena, 75:253-272, 1995.

[70] CT Chen, YU Idzerda, H-J Lin, NV Smith, G Meigs, E Chaban, GH Ho, E Pellegrin, and F Sette. Experimental confirmation of the X-ray magnetic circular dichroism sum rules for iron and cobalt. Physical review letters, 75(1):152, 1995.

[71] Tobias Funk, Aniruddha Deb, Simon J George, Hongxin Wang, and Stephen P Cramer. X-ray magnetic circular dichroisma high energy probe of magnetic properties. Coordination Chemistry Reviews, 249(1):3-30, 2005.

[72] Félix Jiménez-Villacorta, C Prieto, Yves Huttel, ND Telling, and G van der Laan. $\mathrm{X}$-ray magnetic circular dichroism study of the blocking process in nanostructured iron-iron oxide core-shell systems. Physical Review B, 84(17):172404, 2011.

[73] Akane Agui, Ryo Masuda, Yasuhiro Kobayashi, Tadashi Kato, Shun Emoto, Kosuke Suzuki, and Hiroshi Sakurai. The study of the magnetization process of fe film by magnetic compton scattering and mössbauer spectroscopy. Journal of Magnetism and Magnetic Materials, 408:41-45, 2016.

[74] NM Shumeiko and JG Suarez. The qed lowest-order radiative corrections to the two polarized identical fermion scattering. Journal of Physics G: Nuclear and Particle Physics, 26(2):113, 2000.

[75] A Ilyichev and V Zykunov. Lowest order qed radiative corrections to longitudinally polarized møller scattering. Physical Review D, 72(3):033018, 2005.

[76] Andrei Afanasev and Alexander Glamazdin. Atomic electron motion for møller polarimetry in a double-arm mode. arXiv preprint hep-ex/9602002, 1996. 